\title{
Structure, Dynamics and Phase Behaviors of Cationic Micellar Solutions:
}

\author{
Raman and Neutron Scattering Study of \\ Alkyltrimethylammonium Bromides
}

Dissertation zur Erlangung des Doktorgrades der Mathematisch-Naturwissenschaftlichen Fakultäten der Georg-August-Universität zu Göttingen

vorgelegt von

Chandrashekara Rajashekara Haramagatti

Aus Malligara, India

Göttingen 2006 
D7

Referent: Prof. Dr. G. Eckold

Korreferent: Prof. Dr. C. Griesinger

Tag der mündlichen Prüfung: 01.11.2006 
To my beloved parents 


\section{Contents}

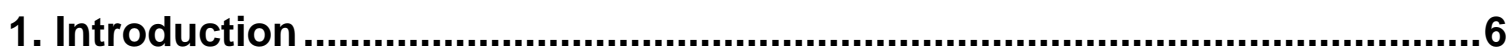

2. Surfactant systems

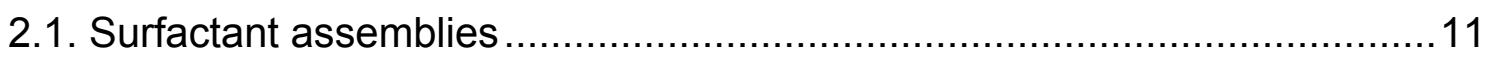

2.1.1 Critical Micelle Concentration $(\mathrm{cmc})$ and Micelles .............................11

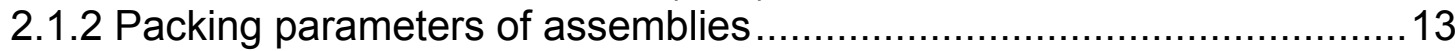

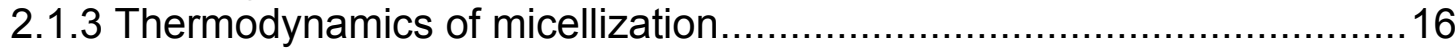

2.2 Effect of temperature, alkyl chain length, and added electrolyte on $\mathrm{cmc} \ldots . .18$

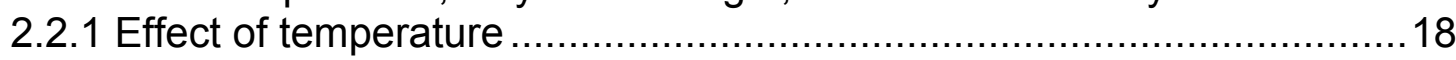

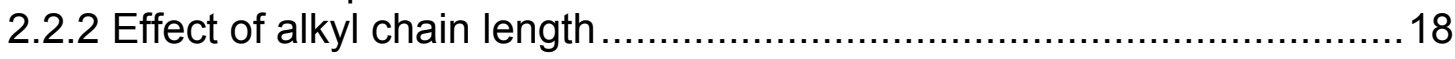

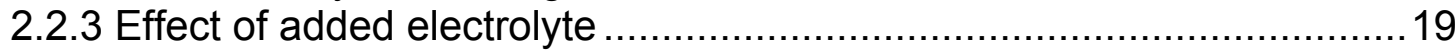

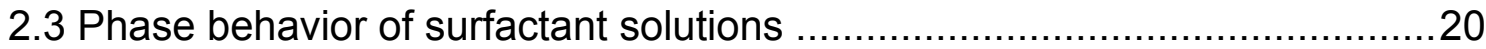

3. Experimental techniques and Surfactant systems used .........................23

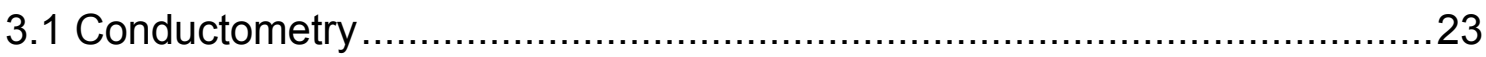

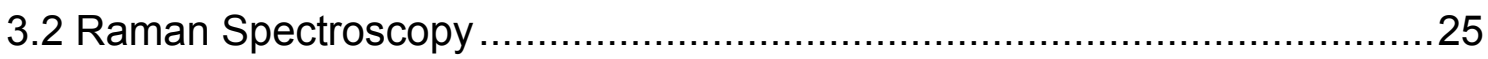

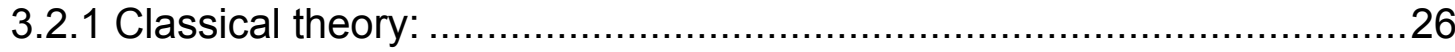

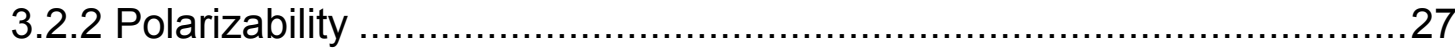

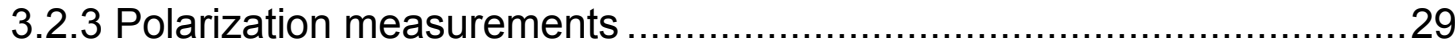

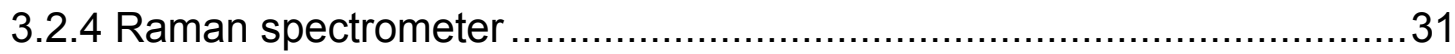

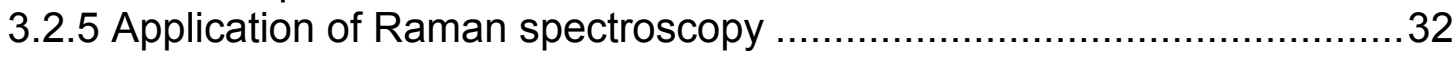

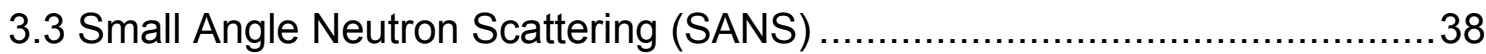

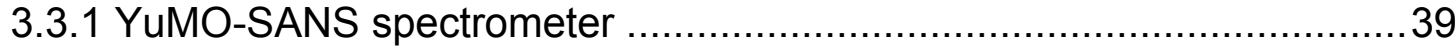

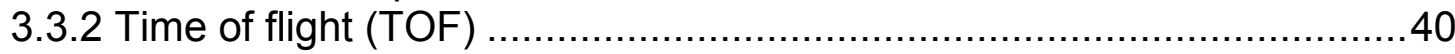

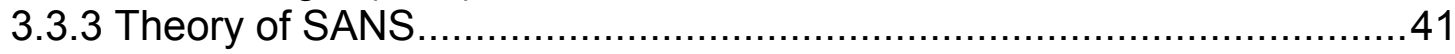

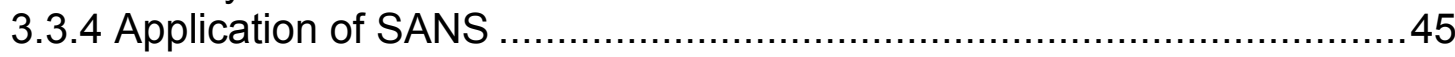

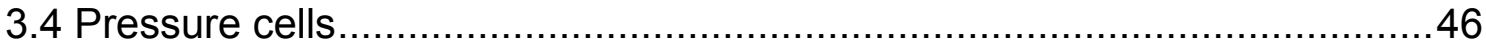

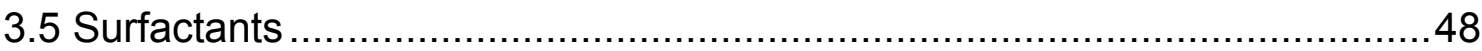

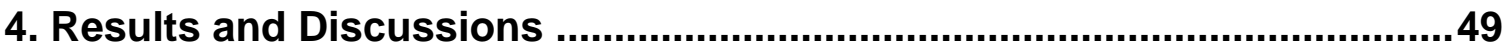

4.1 Critical micelle concentration ( $\mathrm{cmc}$ ) determination ................................49

4.2 Aggregation behaviors at ambient conditions.........................................50

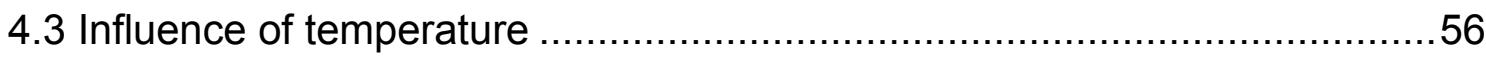

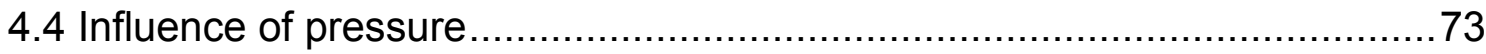

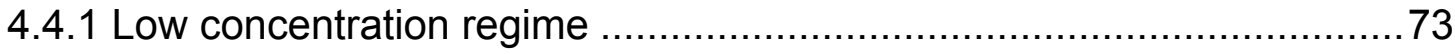

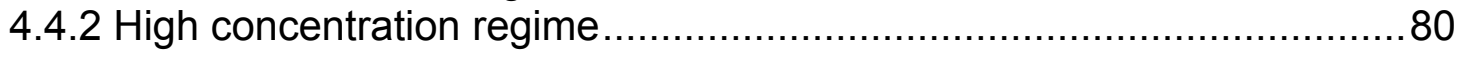

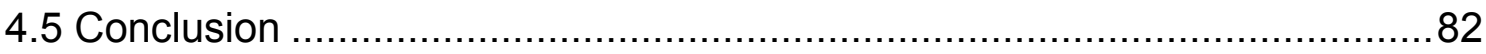

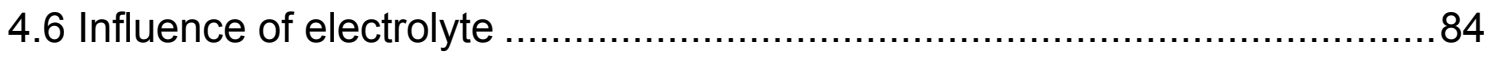

4.6.1 Influence of added electrolyte on TTAB at ambient conditions; .............84 
4.6.2 Influence of added electrolyte, on phase transition; with applied pressure

4.6.3 Influence of added electrolyte, on phase transition; at ambient pressure

.

4.7 Influence of alkyl chain length at ambient conditions ...............................95

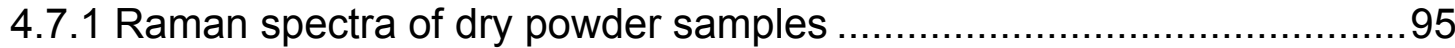

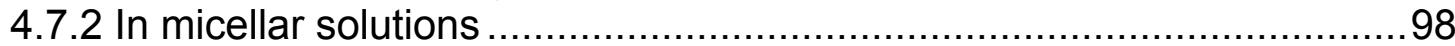

4.7.3 Thermal behavior of Octadecyltrimethylammonium Bromide (OTAB) 102

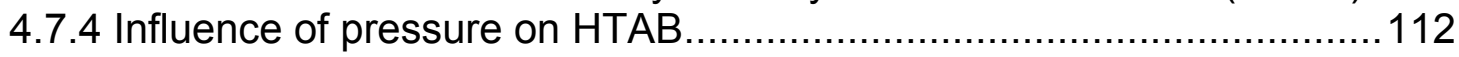

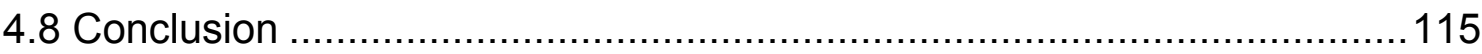

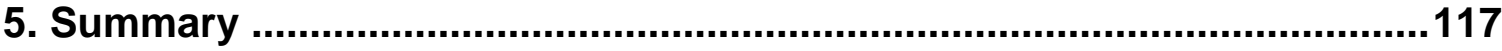

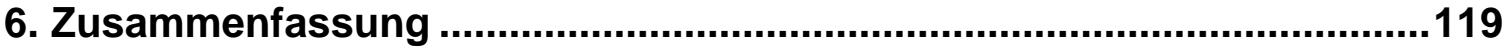

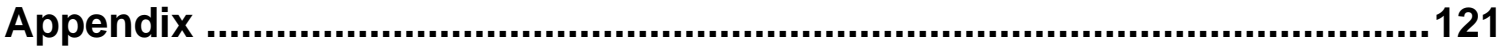

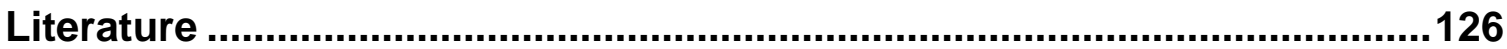

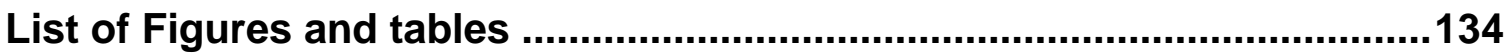

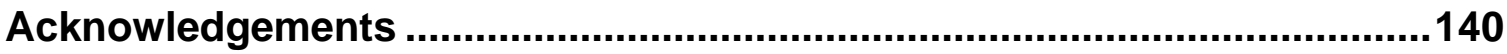

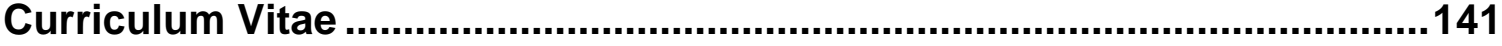




\section{Introduction}

The aggregation behaviour of surfactant molecules with hydrophilic head group and hydrophobic tails in aqueous solutions has received enormous interest in the past. This is not only due to the technical importance of colloid and complex fluid systems but also to the variety of aggregation phenomena that can be studied, both theoretically and experimentally. The hydrophobic interaction between surfactant molecules leads to the formation of micelles [Tanford_1973] as soon as the surfactant concentration exceeds the critical micelle concentration $(\mathrm{cmc})$. All micelles are believed to have the same size and shape. $\mathrm{cmc}$ is known to depend on the molecular structure (size of the head group, length of the hydrophobic tail), as well as on temperature and pressure [Shinoda_1963]. All molecules within a micelle looses their individual character and hence, a micelle is taken to be composed of $\mathrm{N}_{\text {agg }}$ times an average molecule. Consequently, all micelles can be characterized by simple geometric parameters (like the size/aggregation number and shape), which are experimentally obtainable by suitable methods (measuring the form factor) like dynamical light scattering (DLS) or small angle neutron scattering (SANS). In a huge number of investigations it was found that both aggregation number and morphology depends on external thermodynamic parameters, concentration and most importantly in ionic systems like alkyl-trimethyl-ammonium halides, on the amount of electrolyte additives. Moreover, due to long-range Coulomb interaction, charged micelles tend to arrange themselves in an ordered structure that gives rise to a correlation-peak in small angle neutron scattering [Eckold_2001]. Hence, SANS intensity profile as described by the product of a form factor and a structure factor carries information not only about single particle properties but also about the collective structure formed in these complex liquids. At high concentrations, the formations of liquid crystals or hydrated crystals are frequently observed and as soon as the temperature is decreased below the 
Krafft-point, there is a precipitation of hydrated crystals. Consequently, a large variety of phase diagrams have been found in numerous systems [Laughlin_1994].

The vibrational character of the $\mathrm{N}_{\text {agg }}$ surfactant molecules within the micelle can be analysed with spectroscopic techniques like Raman spectroscopy. This is very worthful, since the internal dynamics can change during the sequence of different aggregation states and phase transitions. In the sodium oleate system, Wong et al. were able to show that there are characteristic variations of vibrational spectra [Wong_1983, Wong_1983a] on crossing phase boundaries.

With decrease of temperature the phase transition from micelles to solid/coagel phase is observed for many surfactant systems [Brown_1987, Wong_1983a]. Fisch and Benedek found a decrease of the mean hydrodynamic radius of sodium dodecylsulfate (SDS) micelles with increasing pressure [Fisch_1986] while in non-ionic surfactants Baden et al. recently observed a minimum of the aggregation number at pressures of about 2 kbar [Baden_2002]. Pressure induced phase transitions in selected systems have been investigated using Raman spectroscopy by Wong et al. [Wong_1983]. SANS data on the tetradecyldimethylaminoxide (TDMAO) micellar system at elevated pressures are reported by Gorski et al. [Gorski_1999] who pointed out particularly that the pressure induced transition is rather sluggish.

It is well know that addition of electrolyte leads to the pronounced growth of micelles that become cylindrical or worm like in shape with characteristic lengths of several $100 \AA$ in solution of large ionic strength [Eckold_2001, Imae_1986]. Different counter ions seem to be highly specific in effect that might be due to their respective hydration behaviour [Aswal_2003].

Surfactant, tetradecyltrimethylammonium bromide (TTAB) shows phase transition from micelles to solid phase on cooling or with applied pressure. The higher homologous $(>\mathrm{C} 14)$ series of surfactant molecule, alkyltrimethylammonium halides shows greater influence on geometrical parameters. The Krafft temperature increases with the increase of alkyl chain 
length [Davey_1998]. The metastable gel phase is commonly observed in such systems with the application of temperature [Kaneshina_1980, Kodama_1986].

This thesis presents the systematic investigations of the structure, dynamics and the phase behaviors of the aqueous micellar solutions of alkyltrimethylammonium bromide at different thermodynamic conditions. The primary motivation for this study is to better understanding of the behavior of surfactant molecules under different conditions of surfactant concentration, alkyl chain length, temperature, pressure and added salt. For the first time, Raman spectroscopy and Small Angle Neutron Scattering with their specific scope have been used to combine structural and vibrational characteristics.

Chapter 2 will give an idea about the amphiphilic/surfactant molecules, aggregation of these surfactant molecules in water, when they aggregate, possible patterns of aggregation, the geometrical parameters that determine the aggregation pattern and the thermodynamics of aggregate type micelles. Dependence of temperature, alkyl chain length and added electrolyte on the critical micelle concentration is also discussed.

Chapter 3 presents surfactant systems along with experimental techniques, principles and application of the techniques employed to characterize the aggregates of the surfactant molecules in water. The conductivity meter has been used to measure the critical concentration of the surfactant to form the micelle.

Raman spectrometer has been used to understand the dynamical behavior of the aggregates. A brief introduction to the Raman discovery is given along with the classical theory of the Raman scattering. This is followed by the literature survey for application of the Raman spectrometer in understanding the conformation of the $\mathrm{n}$-paraffins and surfactants of the aggregates of various kinds.

SANS has been used to analyze the structure of the aggregates, which gives the size, charge and aggregation number of the aggregate (micelle). The important 
terms of the SANS measurements, the cross section, structure factor and form factor are also described. The structural information of the aggregates is given by the form factor and the structure factors. The data analysis is also explained briefly. The use of SANS by other authors in studying similar surfactant systems has been discussed.

Chapter 4 is dedicated to the results and discussion. The first part of this chapter is focused on a detailed study of TTAB under thermodynamic parameters such as temperature and pressure. The second part concentrates the effect of added electrolyte on micellar solution of TTAB under thermodynamic parameters. The influence of alkyl chain length on geometrical parameters of the micelles and the associated phases is also presented.

Chapter 5 Summary

Chapter 6 Zusammenfassung

Appendix, literature, and list of figures and tables are presented at the end. 


\section{Surfactant systems}

Surfactants are amphiphilic molecules which have both hydrophilic (water loving) and hydrophobic (water hating) parts. In early days, amphiphiles were known as amphipathy, the word amphipathy (the greek, "amphi" means of both kinds, "pathos" means feeling) indicates the possession of both feelings was first proposed by Hartley [Hartley_1936], who investigated the properties of aqueous solutions of paraffin salts almost 8 decades ago. Later, the word amphipathy was replaced by amphiphile ("philos" means strong affinity or attraction, or love). This term describes the presence of both hydrophilic and hydrophobic parts in the same molecule. The hydrophilic part (sometime called the head group of the amphiphile) can be either charged (anionic, cationic, zwitterionic) or nonionic/polar (polyoxyethylene chain, amine oxide, etc). The hydrophobic part is generally hydrocarbons, but can be partly or completely halogenated as in the fluorocarbon amphiphiles. A schematic representation of a cationic amphiphile is shown in Figure 2.1 with a trimethylammonium head group and a hydrophobic part with an alkyl chain length of 14 carbon atoms.

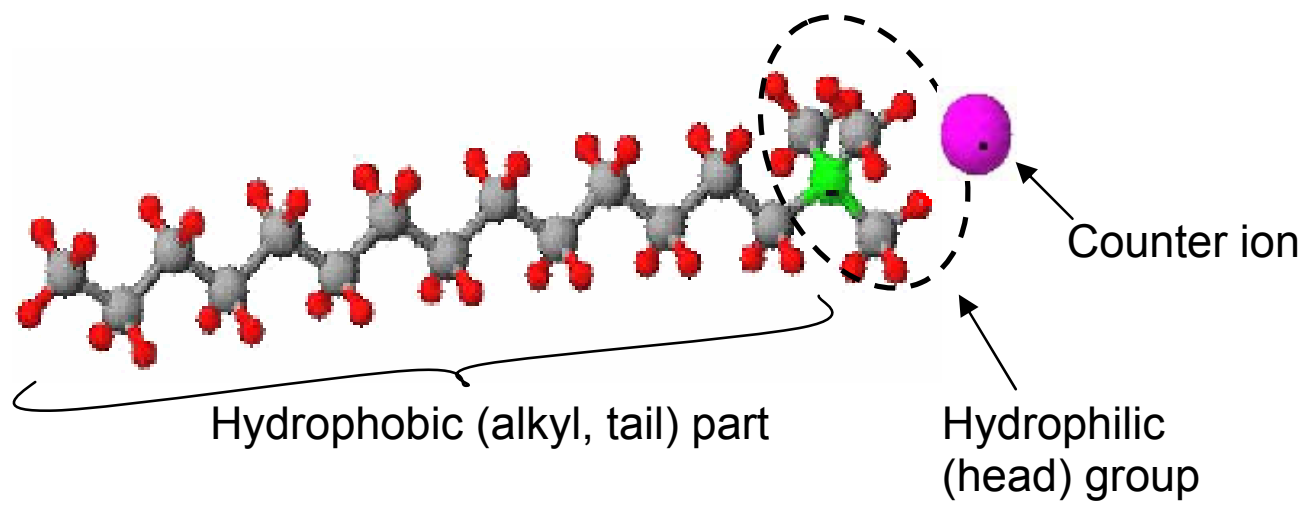

Figure 2. 1: Schematic representation of an amphiphile, the part which is inside the dotted oval is hydrophilic (head) group and a counter ion, and the rest hydrophobic part

They can consist of one or two linear (or branched) chains, and they can contain unsaturated portions or aromatic moieties. Such amphiphilic molecules show 
differences in their properties when they are in bulk and at surface. They can form monolayers at air-water or oil-water interfaces. Since the partition coefficient between bulk and surface is very large in aqueous amphiphilic solutions, a very low concentration of amphiphile can reduce very effectively the surface tension. For this reason the amphiphiles are also known as surfactants or surface active agents.

\subsection{Surfactant assemblies}

The surfactant aggregation is mainly driven by hydrophobic and electrostatic interactions [Tanford_1973]. Concentration of the surfactant solution determines the type of assemblies. Higher surfactant concentrations lead to the ordered assemblies like cubic, hexagonal or lamellar phases whereas the lower concentrations lead to many disordered phases [Rubingh_1991, Laughlin_1994].

\subsubsection{Critical Micelle Concentration (cmc) and Micelles}

The concentration boundary above which the association of surfactants starts to give spherical objects known as micelles is termed as critical micelle concentration (cmc) [Shinoda_1963]. Below this concentration the surfactants are present as monomers (single surfactant molecule) spread over the solution. Such monomers can also give certain ordered structures; usually self assembled monolayers (SAMs) on mica or metallic surfaces formed by the simple adsorption methods (for example SAMs of alkane thiols/alkyltrimethylammonium bromides on mica, single/poly crystalline gold and stainless steel surfaces) [Fan_1997]. The self assembled monolayer systems play a very important role in the field of boundary lubrication, corrosion inhibition, electrochemical sensors etc [Patrick_1999, Prathima_2005]. 
The micelles are formed above the $\mathrm{cmc}$ of the aqueous surfactant solution [Shinoda_1963]. The hydrophobic part of the surfactant molecules is inside the micelle (known as core of the micelle) and the hydrophilic part is at the surface. There are also some systems where, the hydrophobic part of the surfactant molecules is outside and the hydrophilic part is inside the micelle, such systems are known as inverse micelles.

The name micelle (from the Latin, "micelle" means small bit) was introduced by McBain [McBain_1913], but aggregation mechanism and the first geometrical model of the spherical micelle was proposed by Hartley [Hartley_1936]. The McBain and Hartley models are shown in Figure. 2.2

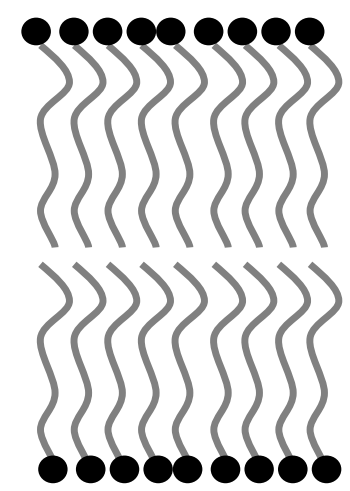

Figure 2. 2 : The Mc Bain lamellar micelles

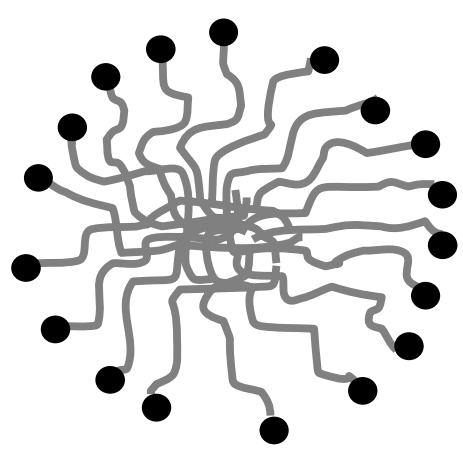

The Hartley spherical micelles

According McBain, there are two kinds of micelles, one formed below the cmc with not more than 10 surfactants and they have retained their charges, the other is a micelle with little or no ionic charge, formed just above the $\mathrm{cmc}$, so that the solution contains both highly charged and partially charged lamellar micelles. Later, Hartley [Hartley_1936] proposed a simpler spherical micelle model. Up to the $\mathrm{cmc}$ the surfactants behave as a strong electrolyte, completely dissociated. At the cmc the surfactants begin to aggregate; at first they form small micelles which grow rapidly over a very limited concentration range. Hartley believed that the micelles are liquid and essentially spherical and their interior part approximate the random distribution of liquid paraffin, but the hydrophilic part of the surfactant remain at the surface of the micelles. Thus, Hartley postulated only one type of micelles, of approximately constant size for a given surfactant. 


\subsubsection{Packing parameters of assemblies}

Formation of the type of assemblies depends on the geometry of the single surfactant molecule which is well represented by packing parameter ' $\mathrm{P}_{\mathrm{P}}$ ' defined as

$$
\mathrm{P}_{\mathrm{P}}=\frac{\mathrm{V}}{\mathrm{al}}
$$

Where ' $\mathrm{V}$ ' is the volume of single surfactant, ' $\mathrm{a}$ ' is the cross sectional area per surfactant molecule and ' $\mathrm{l}$ ' is the length of the fully extended chain.

The packing parameter ' $\mathrm{P} \mathrm{p}$ ' can be evaluated by the aggregation number, which is the ratio of the volume of the micelle to the volume of the single surfactant ' $\mathrm{V}$ '

$$
\mathrm{N}=\frac{\frac{4}{3} \pi \mathrm{R}_{\text {mic }}^{3}}{\mathrm{~V}}
$$

where ' $R_{\text {mic }}$ ' is the radius of the spherical micelle. The aggregation number is also given by the ratio of the area of the micelle (assuming that the surface is completely covered with head groups of the micelle) to the cross-sectional area per surfactant molecule ' $a$ ':

$$
\mathrm{N}=\frac{4 \pi \mathrm{R}_{\text {mic }}^{2}}{\mathrm{a}}
$$

Equating (2.2) and (2.3), we get

$$
\frac{\mathrm{V}}{\mathrm{aR}_{\text {mic }}}=\frac{1}{3}
$$

The radius of the micelle ' $R_{\text {mic }}$ ', cannot exceed the length of the fully extended chain 'l', so equation 2.4 can be written as

$$
\mathrm{P}_{\mathrm{P}}=\frac{\mathrm{V}}{\mathrm{al}} \leq \frac{1}{3}
$$




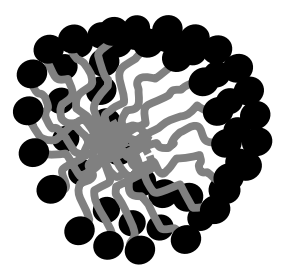

$$
\mathrm{P}_{\mathrm{P}}<\frac{1}{3}
$$

Spherical micelles

$$
\frac{1}{3}<\mathrm{P}_{\mathrm{P}} \leq \frac{1}{2}
$$
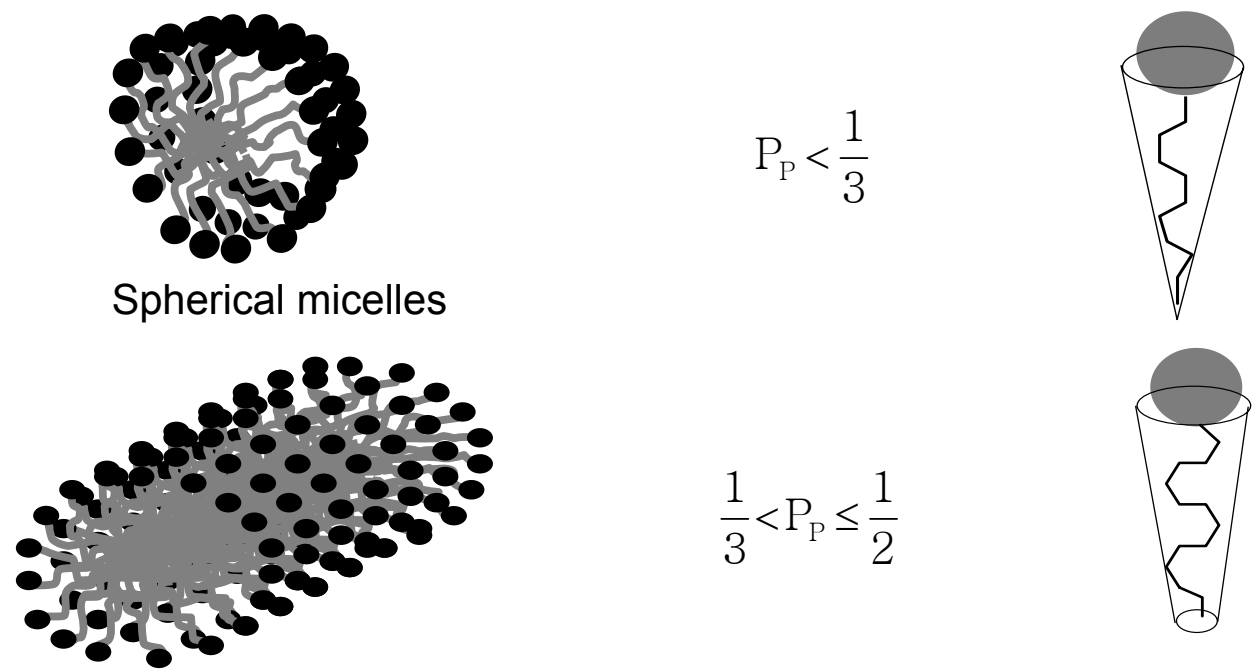

Cylindrical micelles

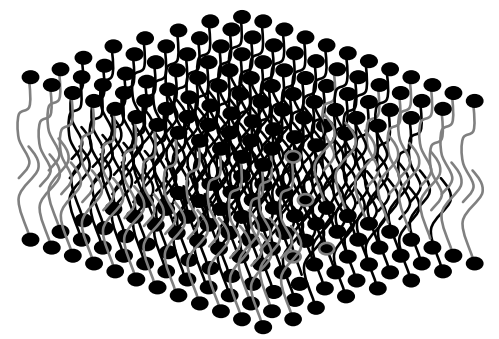

Bilayer

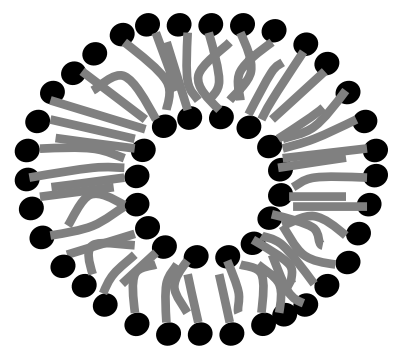

$\frac{1}{2}<\mathrm{P}_{\mathrm{P}} \leq 1$

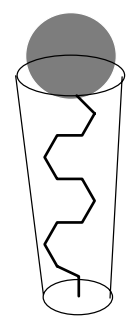

vesicles
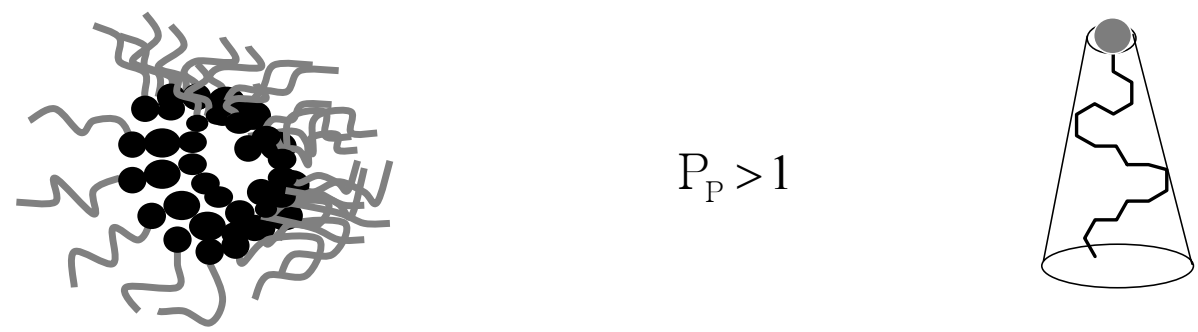

Inverse micelle

Table 2. 1: Showing the packing parameters for different aggregates 
The aggregates which do not exhibit spherical shapes are characterized by packing parameters larger than $1 / 3$. The surfactant packing parameter range and the various surfactants aggregate shapes are compared in Table 2.1 (see above). The spherical micelles can be considered to be built from the packing of cones, corresponding to effective molecular volumes (large difference between the cross-sectional area of the head group and tail group). Other aggregate of type cylinder micelles formed by surfactants with truncated cones (with packing parameters $1 / 3<\mathrm{P}_{\mathrm{P}}<1 / 2$ ). The bilayers and vesicles result from a packing of surfactants with almost equal of cross sectional area of head group and tail group $\left(1 / 2<\mathrm{P}_{\mathrm{P}} \leq 1\right)$. Exceeding $1\left(\mathrm{P}_{\mathrm{P}}>1\right)$ results in inverse micelles.

Apart from these aggregate shapes, many authors [Tarter_1955, Tanford_1972] have examined that the surfactant molecules can also pack into prolate and oblate spheroids. Figure 2.3 gives the schematic view of the prolate and oblate spheroids. The semi major axis ' $a$ ' is larger than the semi minor axis ' $b$ ' in case of the prolate spheroids. The length of the semi minor axis is less than the length of the fully extended alkyl chain of the surfactant molecule. In case of oblate spheroids the semi major axis ' $a$ ' is smaller than the semi minor axis ' $b$ '.

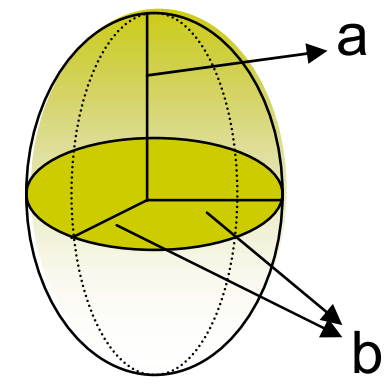

(i) Prolate ellipsoid

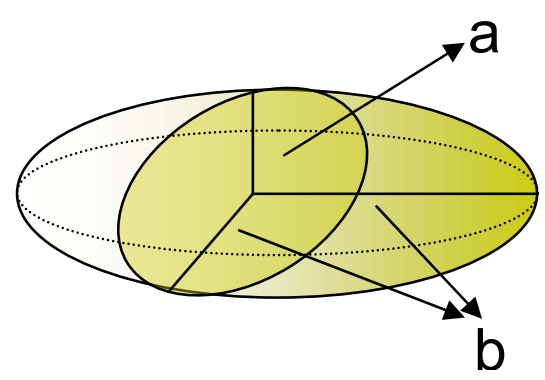

(ii) Oblate ellipsoid

Figure 2. 3: Schematic representation of the models of prolate (i) and oblate (ii) shperoids, ' $a$ ', the major semi-axis and the 'b', the minor semi-axis 


\subsubsection{Thermodynamics of micellization}

The thermodynamic formulation of micellization follows two methods namely mass action model and the second one which considers the surfactant solution as two phase system (or phase separation model), where the micelles are treated as separate phase. A comparison of phase separation model and mass action model for the calculation of Gibbs energies of micelle formation is well documented by Blandamer et al [Blandamer_1995].

First, consider the phase separation model, in such cases the Gibbs phase rule would apply and micelles are in dynamic equilibrium with surfactant monomer in the bulk solution. For a given temperature and pressure there is a steady exchange of the surfactant molecule in the micelle and in the monomer solution. Phase separation model assumes complete binding of counter ions to the micelles. The formation of micelles of ionic surfactant (for example, alkyltrimethylammonium bromides) at equilibrium is written as;

$$
\begin{array}{cc}
\left.\mathrm{N}\left(\mathrm{RN}^{+} \mathrm{Me}_{3} \mathrm{Br}\right)^{-}\right) & \left(\mathrm{RN}^{+} \mathrm{Me}_{3} \mathrm{Br}^{-}\right)_{\mathrm{N}} \\
\text { (surfactant) } & \text { (micelles) }
\end{array}
$$

At equilibrium the chemical potentials of substances are equal

$$
\mathrm{N} \mu_{\text {sol }}\left(\mathrm{RN}^{+} \mathrm{Me}_{3} \mathrm{Br}^{-}\right)=\mu_{\text {mic }}\left(\mathrm{RN}^{+} \mathrm{Me}_{3} \mathrm{Br}^{-}\right)_{\mathrm{N}}
$$

(in solution at $\mathrm{cmc}$ ) (micelles at $\mathrm{cmc}$ )

where ' $\mu_{\text {sol }}$ ' and ' $\mu_{\text {mic }}$ ' are chemical potentials of surfactants in solution and micelles. For a homogeneous solution, where micelles are dispersed in solution with aggregation number ' $\mathrm{N}$ ', the major contribution per mole of surfactant would be $1 / \mathrm{N}$ of the contribution per mole of the micelles. The mole fraction of surfactant incorporated into micelles ' $X_{\text {mic }}$ ' (note, $X_{\text {mic }}$ is the mole fraction of surfactant molecule present in the micelles and not the mole fraction of micelle) would be $\mathrm{N}$ times the mole fraction of micelles. This can be expressed as:

$$
\mu_{\mathrm{mic}}=\mu_{\mathrm{mic}}^{0}+\frac{\mathrm{RT}}{\mathrm{N}} \ln \frac{\mathrm{X}_{\mathrm{mic}}}{\mathrm{N}}
$$


where ' $\mu_{\text {mic }}^{0}$ ' is the standard chemical potential of surfactants in micelles, ' $R$ ' is the gas constant and ' $\mathrm{T}$ ' is the temperature.

Mass action model, considers that the fraction of counter ions are bound to the micelles and is better suited for modeling ionic surfactants.

At equilibrium the micelle formation is written as,

$$
\mathrm{N}\left(\mathrm{RN}^{+} \mathrm{Me}_{3} \mathrm{Br}{ }^{-}\right) \rightleftharpoons\left\{\mathrm{N}\left(\mathrm{RN}^{+} \mathrm{Me}_{3}(\mathrm{~N}-\alpha) \mathrm{Br}^{-{ }^{\alpha+}}\right)^{-}\right\}+\alpha \mathrm{Br}^{-}
$$

(surfactant)

$$
\text { (micelles) }
$$

(counter ions)

This process can be described using thermodynamic variables characterizing the standard states of the surfactant and micelles in solution. Then ' $\Delta_{\text {mic }} G$ ', is the standard Gibbs energy of micelle formation from $\mathrm{N}$ moles of surfactant $\left(\mathrm{RN}^{+} \mathrm{Me}_{3} \mathrm{Br}\right)$. In terms of equilibrium constant for micelle formation this quantity is written as;

$$
\Delta_{\text {mic }} \mathrm{G}^{0}=-\mathrm{R} T \ln \mathrm{K}
$$

From the temperature dependence of $\mathrm{cmc}$ one can determine the ' $\Delta_{\mathrm{mic}} \mathrm{G}$ ',

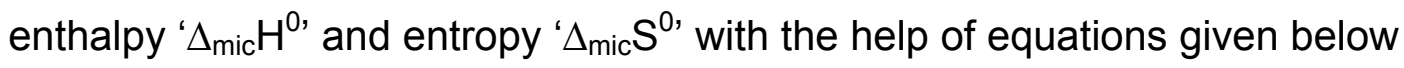

$$
\begin{aligned}
& \Delta_{\text {mic }} G^{0}=R T \text { In } c_{c m c}
\end{aligned}
$$

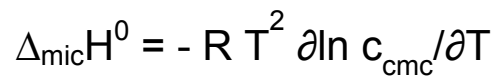

$$
\begin{aligned}
& \Delta_{\text {mic }} S^{0}=\left(\Delta_{\text {mic }} H-\Delta_{\text {mic }} G^{0}\right) / T
\end{aligned}
$$

Determination of more accurate values of these thermodynamic terms is achieved by calorimetric experiments. Stodghill [Stodghill_2004] has tabulated all these thermodynamic values for alkyltrimethylammonium bromides (DTAB, TTAB and $C T A B)$ from the previously reported results. The enthalpy $\left(\Delta_{\text {mic }} H^{0}\right)$ decreases with increase in temperature and with increase of alkyl chain length. The Gibbs free energy is negative as expected for spontaneous micellization near the cmc. Gibbs free energy becomes more negative with increase of temperature. The term entropy decreases with increase in temperature and increases with increase in alkyl chain length. 


\subsection{Effect of temperature, alkyl chain length, and added electrolyte on $\mathrm{cmc}$}

\subsubsection{Effect of temperature}

The effect of temperature on the $\mathrm{cmc}$ of the ionic and zwitterionic micelles is very small. In other words, for ionic surfactants, the cmc is almost independent of temperature. However, nonionic surfactant solutions show dependence of temperature. Aqueous solutions of nonionic surfactants suddenly turns to cloudy (turbid) when the temperature is elevated to a certain temperature which is known as "cloud point", and a two phase system can be seen just above this temperature, this phenomenon is due to the dehydration of head groups at higher temperature [Kumar_2000]. Work of Nakagawa and Shinoda [Shinoda_1963] suggests that below the cloud point $\mathrm{cmc}$ decreases and the aggregation number increases with increase of temperature for a nonionic surfactant methoxypolyoxyethylene octanoate $\left(\mathrm{C}_{7} \mathrm{H}_{15} \mathrm{COO}\left(\mathrm{CH}_{2} \mathrm{CH}_{2} \mathrm{O}\right)_{7.6} \mathrm{CH}_{3}\right.$, cloud point is $\left.44^{\circ} \mathrm{C}\right)$.

\subsubsection{Effect of alkyl chain length}

Increase of alkyl chain length of the hydrophobic part of the surfactant molecule favours the micellization. Thus an increase in the chain length leads to a reduction in the cmc. The number of carbon atom ' $m$ ' in the alkyl chain and the cmc are expressed by an equation [Klevens_1953]

$$
\log _{10} c_{c m c}=A-B m
$$

where ' $A$ ' and ' $B$ ' are constants for the particular homologous series and temperature [Shinoda_1963]. For a wide range of surfactants, the constants A and $B$ are in the ranges from $1.4-1.8$ and $0.22-0.30$, respectively. This equation (2.14) holds good for the chains containing up to 16 carbon atoms. Increasing the chain length beyond this number does not lead to significant 
decrease in the $\mathrm{cmc}$, the possible reason would be due to the coiling of the monomer to minimize hydrophobic interactions of unassociated molecules. Using thermodynamic equation which relates Gibbs free energy ' $\Delta_{\text {mic }} \mathrm{G}$ ', and the $\mathrm{cmc}$ (equation 2.11) the decrease in the $\mathrm{cmc}$ can be explained. Making comparisons for a homologous series, the contribution to the Gibbs energy per methylene unit, $\Delta_{\text {mic }} \mathrm{G}^{0}\left(\mathrm{CH}_{2}\right)$ is approximately $-3 \mathrm{~kJ} \mathrm{~mol}^{-1}$ for a wide range of surfactant types and the corresponding decrease in $\Delta_{\text {mic }} \mathrm{G}^{0}$ with increasing $\mathrm{m}$ largely accounts for the decrease in the cmc [Hamley_2000]. For the case of nonionic surfactants increase in cloud point and decrease of $\mathrm{cmc}$ is observed for methoxypolyoxyethylene decanoates and dodecanoates [Nakagawa_1963].

\subsubsection{Effect of added electrolyte}

Addition of electrolyte to an aqueous surfactant solution decreases the $\mathrm{cmc}$, this effect is greatest for ionic surfactant solutions. Nonionic and zwitterionic surfactants exhibit a much smaller effect. The observed depression in the cmc of ionic surfactants is due to the reduction of the electrostatic repulsion between charged head groups. For a large variety of surfactants the effect of salt on cmc is available in literature [Corrin_1947, Shinoda_1955, Goddard_1953, Herzfeld_1952, Nakagawa_1963]. It was found that added organic salts depress the $\mathrm{cmc}$ more than inorganic salts. It was found for a surfactant system sodium dodecyl sulfate (SDS) the added inorganic salts (sulfates of $\mathrm{Li}, \mathrm{Na}, \mathrm{K}, \mathrm{Cs}$ ) and organic salts of quaternary ammonium ions, the later depresses the $\mathrm{cmc}$ more than the former. Not only this, the size (effective radius) of the cation of the inorganic salts also plays a very important role. With increase of radius of the cation the $\mathrm{cmc}$ increases, due to the fact that smaller cation can reach closest to the micelles surface screening the electrostatic forces and hence depression in the $\mathrm{cmc}$. However, the same explanation is not true for organic salts where the depression is more even though they have higher radius of cation than the inorganic salts, it is believed that the main reason would be the interaction 
between the organic cation and micelles, with increase of alkyl chain of the cation of the added salt can enter inside the micelles.

In case of nonionic and zwitterionic surfactant a small changes in $\mathrm{cmc}$ is because of the changes in the solvent properties of the aqueous solution for the hydrophobic group. For nonionic surfactants, the cloud point has received much more attention rather than $\mathrm{cmc}$ because the effect of salt is more pronounced near cloud point. The cloud point decreases with addition of inorganic salts [Doscher_1951, Nakagawa_1963].

\subsection{Phase behavior of surfactant solutions}

The transformation of surfactant solutions into different phases, namely micelles and hydrated crystals is dependant on the concentration of the surfactant, temperature, pressure, alkyl chain length and added electrolyte. Alkyl chain length above $\mathrm{C} 14$ shows intermediate metastable gel phase. The term hydrated crystal is also termed as coagel phase or solid phase or crystalline phase. The probable reason to name as hydrated crystal/coagel phase would be because of the presence of a tiny layer $(10 \AA)$ of water in between the bilayers of the surfactants, whereas the gel phase is too a bilayers of the surfactant, but with large amount water $(\approx 1000 \AA)$ in between them [Tsuchiya_1994, Ambrosi_2004]. Tsuchiya also pointed out that hydrocarbon chains in the gel phase are packed in hexagonal lattice and rotate or vibrates along the chain axis, whereas in coagel phase the rotation is restricted and the chains are packed in orthorhombic crystal lattice. A schematic of coagel and gel phase is shown in Figure 2.4 (from his work).

In Figure 2.5, a schematic view of phase diagram of concentration of the surfactant against temperature is presented. The solubility of the ionic surfactant is strongly dependent on temperature. At low temperature the solubility is low, but increases sharply in a narrow range of surfactant as the temperature increases. The point at which the solubility curve meets the cmc curve is termed 
as Krafft point [Laughlin_1994], which defines the Krafft temperature as shown in Fig. 2.5. The term Krafft point or Krafft temperature is named in the honor of its discoverer Prof. Friedrich Krafft [Laughlin_1994].

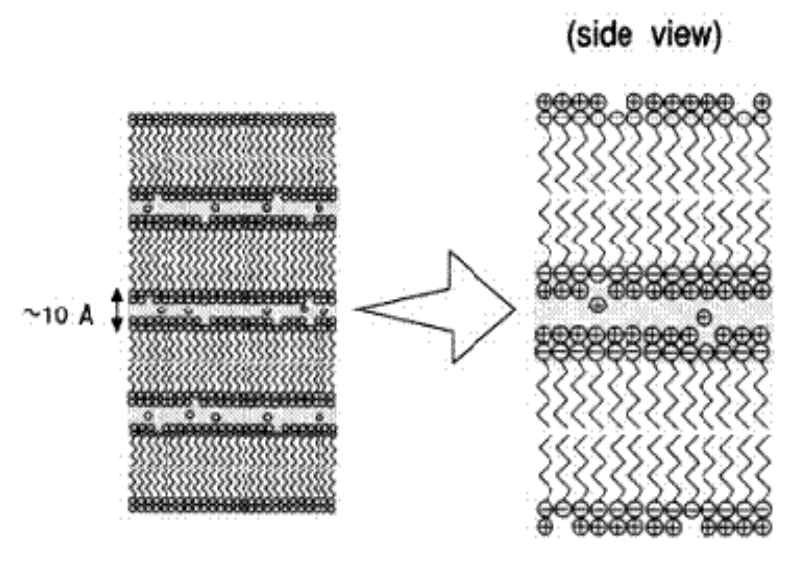

Coagel
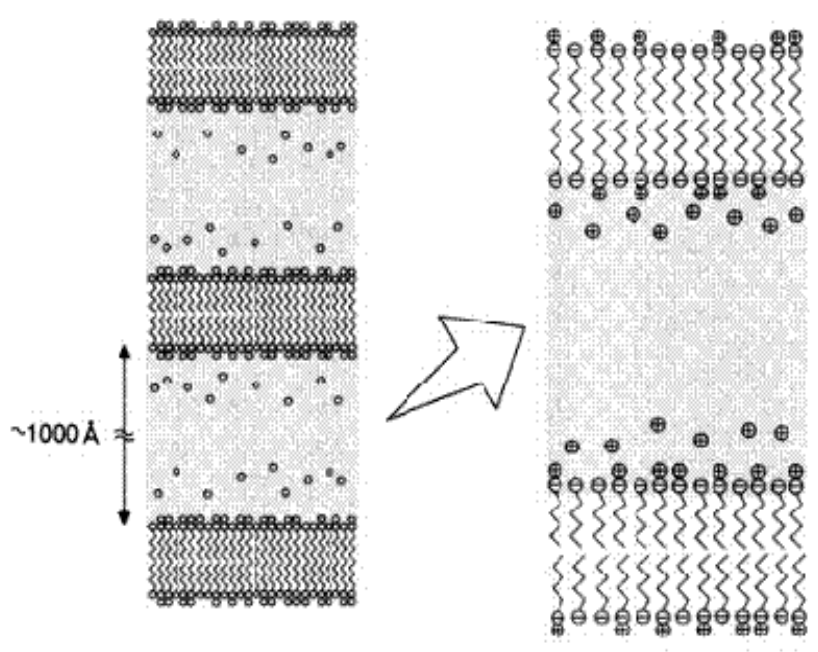

(cross sectional view)

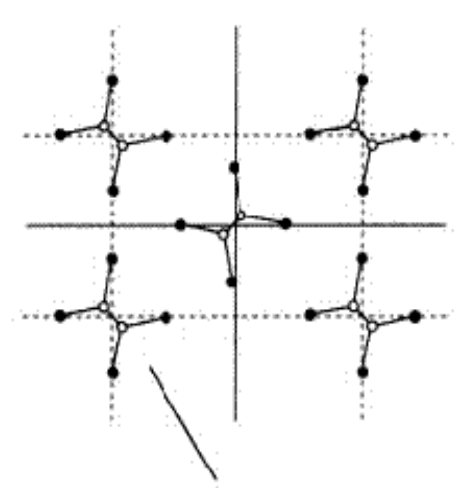

hydrocarbon

chain

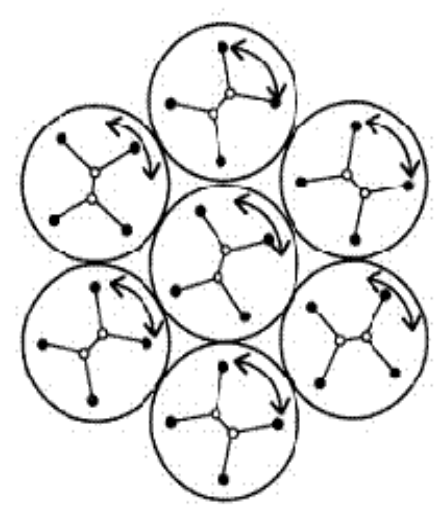

\section{$\mathrm{Gel}$}

Figure 2. 4: Schematic illustrations of the rotational state of surfactant molecules in both the coagel and the gel phase. Open circles and filled circles denote hydrogen atoms and oxygen atoms, respectively, in the cross sectional view (taken from Tsuchiya_1994) 


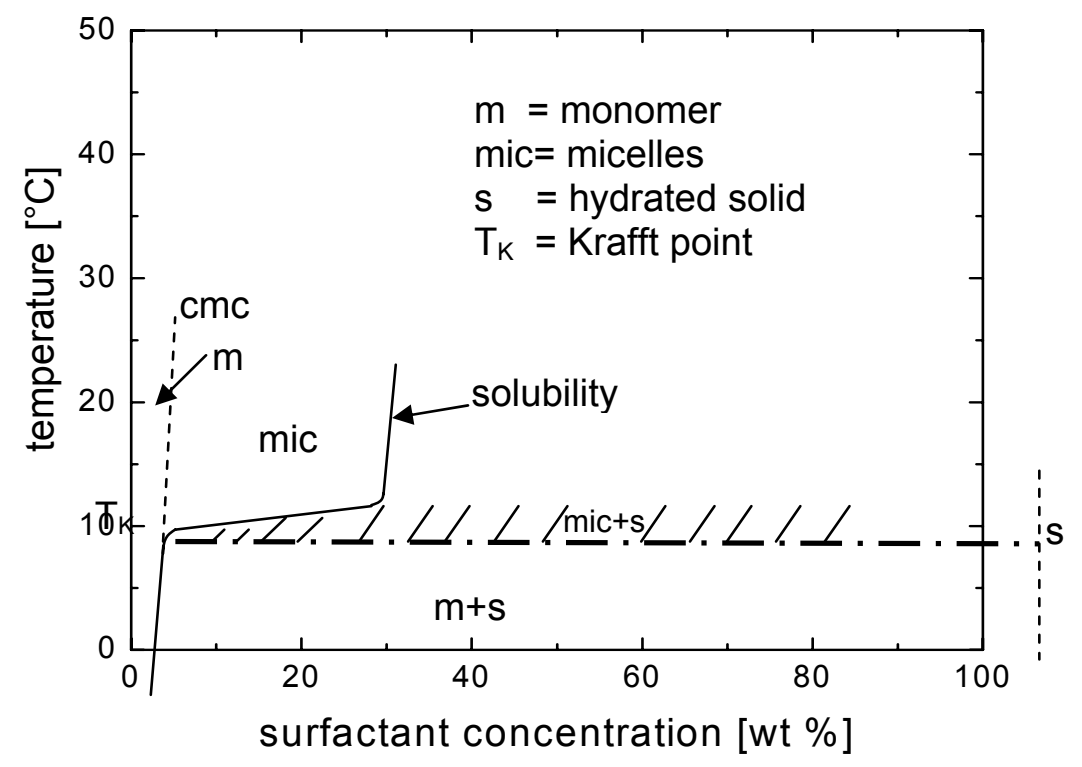

Figure 2. 5: Phase diagram of ionic surfactant

Below the $\mathrm{cmc}$ and the Krafft point, the surfactants exist only in monomer state; micelles forms only above the $\mathrm{cmc}$ and the Krafft point. Hydrated crystal phase can be observed at higher surfactant concentration and low temperatures (below the Krafft point). The Krafft point increases strongly with increase in alkyl chain length of the surfactant. This is mainly due to the dependence of the $\mathrm{cmc}$ on alkyl chain length, which we have discussed already, and also reflects the differences in the packing of surfactant molecules in crystals. Addition of electrolyte also leads to an increase of Krafft point.

The phase transition from micelles to hydrated solid phase is strongly dependant on temperature, pressure, alkyl chain length and added electrolyte. Influence of these parameters on micellar solution of alkyltrimethylammonium bromide will be discussed in next chapters of this thesis. 


\section{Experimental techniques and Surfactant systems used}

\subsection{Conductometry}

The conductivity measurement is a good technique to determine the critical micelle concentration $(\mathrm{cmc})$ of the surfactant solutions, as surfactant molecules dissociates into charged species when added to water.

For the alkyltrimethylammonium bromide surfactant solutions a platinum electrode (from Metrohm) at $25{ }^{\circ} \mathrm{C}$ was used. The cell constant of the electrode was redetermined (done before the sample measurement in order to have precise absolute measurements) using standard $\mathrm{KCl}$ solutions with known conductivity ' $\kappa$ '. For the $\mathrm{cmc}$ determination, first, the stock solution of higher surfactant concentration (100 mM) was prepared, and then the conductivity was measured with diluted solutions. The conductivity ' $\kappa$ ' is measured in units of $\Omega^{-1}$ $\mathrm{cm}^{-1}$. The conductivity depends on the number of charged ions present in the solution, and so in general the conductivity is expressed in molar quantity. If the molar concentration is ' $c$ ', then the molar conductivity is:

$$
\Lambda_{\mathrm{m}}=\kappa / \mathrm{c}
$$

and is expressed in $\Omega^{-1} \mathrm{~cm}^{2} \mathrm{~mol}^{-1}$. The plot of conductivity ' $\kappa$ ' against surfactant concentration is widely used to determine the cmc [Evans_1956]. Figure 3.1 presents the conductivity ' $\kappa$ ' against surfactant (dodecyltrimethylammonium bromide (DTAB)) concentration. The break in the initial slope of the plot gives the $\mathrm{cmc}$, as it is shown here $15.6 \mathrm{mM}$ is the $\mathrm{cmc}$ of DTAB in water at ambient conditions. With a plot molar conductance ' $\Lambda_{\mathrm{m}}$ ' against square root of concentration ' $\sqrt{ } c$ ' (Kohlrausch plot) it is proved that, up to $\mathrm{cmc}$ the solution behaves like a strong electrolyte (Figure 3.2). At low surfactant concentration a straight line behavior is observed below the $\mathrm{cmc}$. The strong electrolytes are well represented by an empirical equation 


$$
\Lambda_{\mathrm{m}}=\Lambda_{\mathrm{m}}^{0}-\mathrm{Kc}^{1 / 2}
$$

where ' $\Lambda_{\mathrm{m}}^{0}$ ' is molar limiting conductance and ' $\mathrm{K}$ ' is an experimental constant.

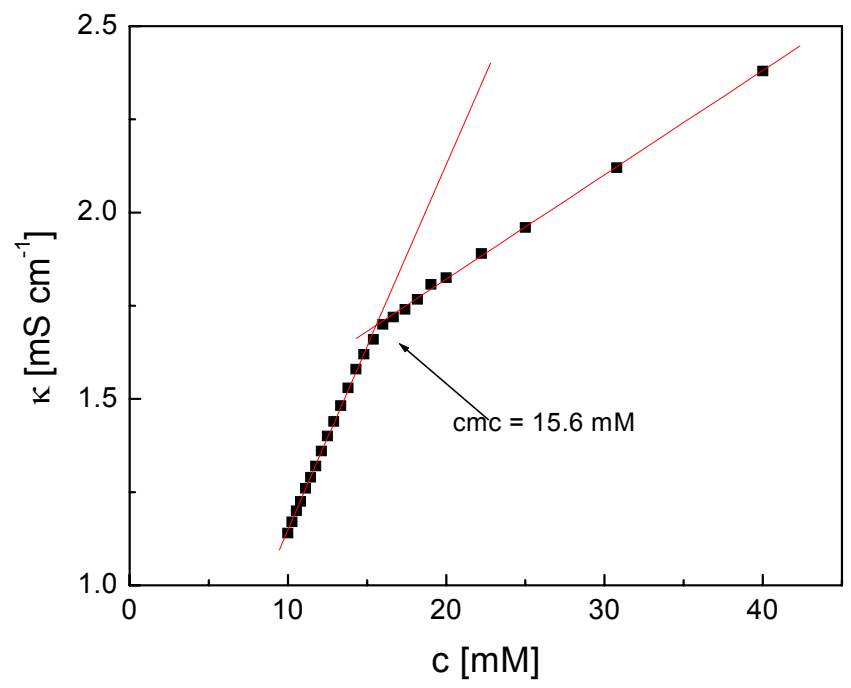

Figure 3. 1: cmc of DTAB determined by conductivity measurements (red lines are linear fit)

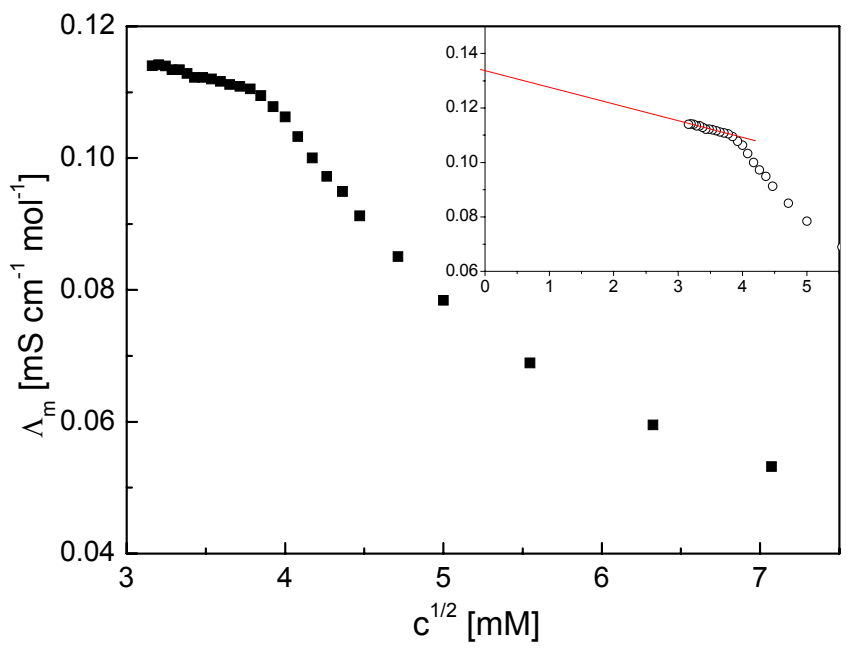

Figure 3. 2: Molar conductance against square root of concentration (inset is the expanded part of lower $\mathrm{x}$-axis scale and red line is linear fit)

Apart from the $\mathrm{cmc}$, the degree of dissociation of the counter ion can be estimated using slopes (below and above the $\mathrm{cmc}$ ) of conductivity plots [Domínguez_1997, Wang_2004]. 


\subsection{Raman Spectroscopy}

This spectroscopic technique is named after the scientist Sir C. V. Raman. He was the first to observe the lines with frequencies other than the incident line frequencies when it passes through the matter. In general, when the incident beam of single, definite frequency, consists of bunch of photons all of with the same energy passes through the sample some of the photons collide with the molecules present in the sample. A large amount of scattered beam will be with the same frequency as before colliding the sample such a scattering is known as Rayleigh scattering named after the physicist Lord Rayleigh. In some cases the photons loose the energy and come out with lower energy. These lower energy photons contribute to lower frequencies called the stokes lines, to the incident/emergent beam. Some of the photons may gain energy from the molecules if they are in excited state, and these contribute to higher frequencies called the anti-stokes line, to the incident/emergent beam. Such an effect was predicted by Smekal in 1923 but it was first observed by C. V. Raman and he received the Noble prize for physics in 1928 [McCreery_2000, Lewis_2001, Gardiner_1989, Wilson_1955]. The Raman scattering can be schematically represented as,

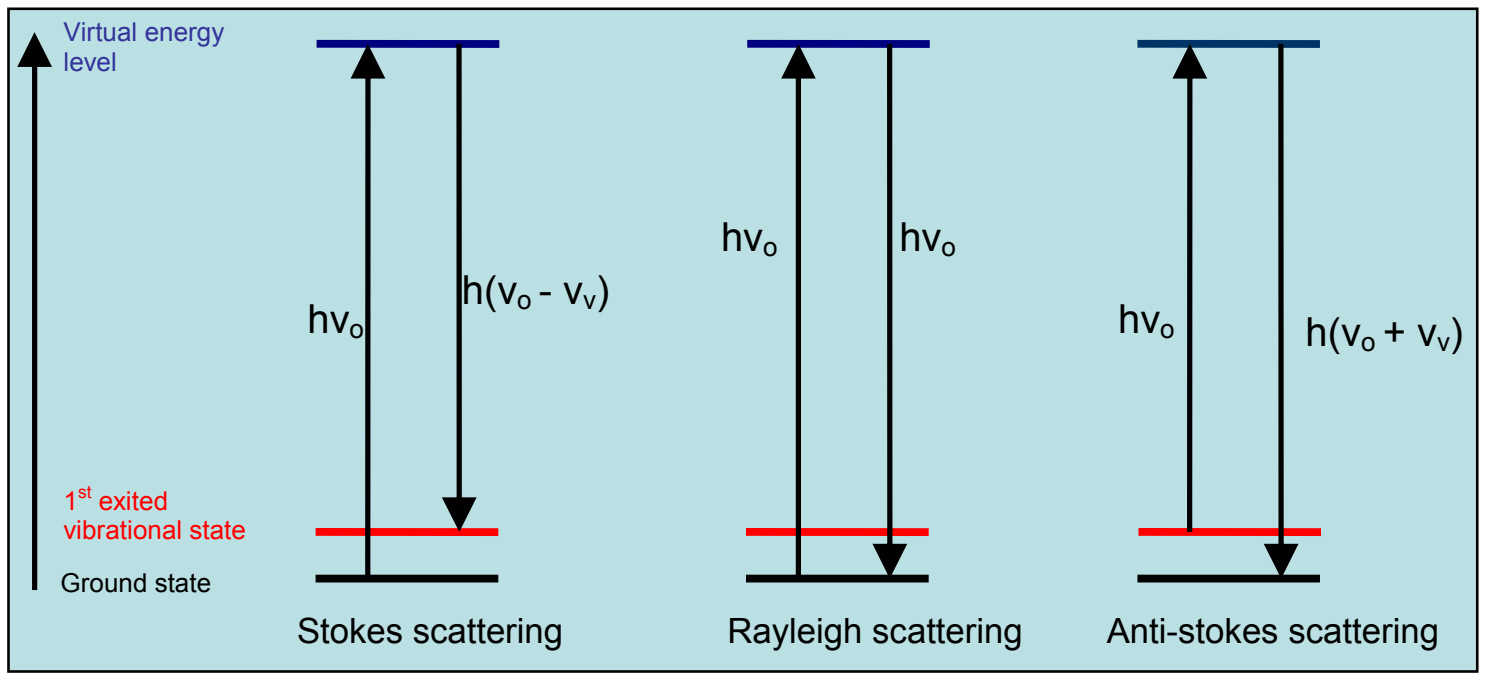

Figure 3. 3: Possibilities of scattering of light; the Rayleigh scattering, stokes (molecules absorb enegry) and anti stokes (molecule release energy) Raman scatterings are shown 


\subsubsection{Classical theory:}

The Raman effect can be well understood with the help of the classical theory, which accounts for the scattering of the molecules undergoing simple harmonic vibrations. The classical description of the Raman scattering is presented in Figure (3.4). When a molecule is placed in an electric field, the oscillating electric field of the incoming light induces the polarization (dipole moment) in the molecule. This induced dipole then radiates the scattered light, with or without exchanging energy with vibrations of the molecule. The induced polarization ' $P$ ' can be represented by the electric field 'E' times the polarizability of the molecule ' $\alpha$ ':

$$
\mathrm{P}=\alpha \mathrm{E}
$$

The fluctuating electric field will produce a fluctuating dipole moment of the same frequency. Electromagnetic radiation generates an electric field which can be written as:

$$
\mathrm{E}=\mathrm{E}_{\mathrm{o}} \cos 2 \pi v_{\mathrm{o}} \mathrm{t}
$$

where ' $\mathrm{E}_{0}$ ' is the equilibrium field strength and ' $v_{0}$ ' is the angular frequency of the molecule.

The molecular vibrations are usually considered to be composed of normal modes ' $\mathrm{Q}_{\mathrm{j}}$ ', of which are $3 \mathrm{~N}-6$ (or $3 \mathrm{~N}-5$ for a linear molecule) in a molecule of $\mathrm{N}$ atoms.

$$
\mathrm{Q}_{\mathrm{j}}=\mathrm{Q}^{\mathrm{o}}{ }_{\mathrm{j}} \cos 2 \pi v_{\mathrm{j}} \mathrm{t}
$$

where ' $v_{\jmath}$ ' is the characteristic harmonic frequency of the jth normal mode. The change in the polarizability during the vibration is given by:

$$
\alpha=\alpha_{\mathrm{o}}+\left(\frac{\delta \alpha}{\delta Q_{j}}\right) Q_{j}+\cdots
$$

Substituting equation (3.5) in equation (3.6) gives:

$$
\alpha=\alpha_{\mathrm{o}}+\left(\frac{\delta \alpha}{\delta \mathrm{Q}_{\mathrm{j}}}\right)_{\mathrm{o}} \mathrm{Q}^{\mathrm{o}}{ }_{\mathrm{j}} \cos 2 \pi \nu_{\mathrm{j}} \mathrm{t}+\cdots
$$


The polarization of the molecule is the product of equation (3.4) and equation (3.7), so we can substitute these in equation (3.3):

$$
\mathrm{P}=\alpha_{\mathrm{o}} \mathrm{E}_{\mathrm{o}} \cos 2 \pi \nu_{\mathrm{o}} \mathrm{t}+\left(\frac{\delta \alpha}{\delta \mathrm{Q}_{\mathrm{j}}}\right)_{\mathrm{o}} \mathrm{E}_{\mathrm{o}} \mathrm{Q}^{\mathrm{o}}{ }_{\mathrm{j}} \cos 2 \pi \nu_{\mathrm{j}} \mathrm{t} \cos 2 \pi \nu_{\mathrm{o}} \mathrm{t}
$$

The second part of the equation (3.8) can be expressed in the form of $\cos a \cos b=[\cos (a+b)+\cos (a-b)] / 2$, the equation (3.8) arrives at,

$P=\alpha_{o} E_{o} \cos 2 \pi v_{o} t+\left(\frac{\delta \alpha}{\delta Q_{j}}\right)_{o} \frac{E_{o} Q^{o} j}{2}\left[\cos 2 \pi\left(v_{o}+v_{j}\right) t+\cos 2 \pi\left(v_{o}-v_{j}\right) t\right]$

The first term in the equation (3.9) corresponds to Rayleigh scattering, which is at the same frequency as the incident light, and has a magnitude proportional to $\alpha_{o}$, the static polarizability of the molecule. The second term $\left(v_{\circ}+v_{\mathrm{J}}\right)$ and the third term $\left(v_{0}-v_{J}\right)$ are the anti stokes and the stokes Raman scattering respectively. The schematic representation of these transitions is shown in Figure 3.3. In addition, for Raman scattering to occur equation (3.9) also demands that the polarizability of the molecule must change.

$$
\left(\frac{\delta \alpha}{\delta Q_{j}}\right)_{0} \neq 0
$$

\subsubsection{Polarizability}

The term polarizability ' $\alpha$ ', which is used in the equation (3.6) is a tensor quantity and as such has components corresponding $x, y, z$ directions of Cartesian coordinate systems. The applied electric component in one direction can induce dipole components in the $x, y$ and $z$ directions as shown in equations (3.11):

$$
\begin{aligned}
& P_{x}=\alpha_{x x} E_{x}+\alpha_{x y} E_{y}+\alpha_{x z} E_{z} \\
& P_{y}=\alpha_{y x} E_{x}+\alpha_{y y} E_{y}+\alpha_{y z} E_{z} \\
& P_{z}=\alpha_{z x} E_{x}+\alpha_{z y} E_{y}+\alpha_{z z} E_{z}
\end{aligned}
$$


These terms can be presented in the matrix notation as:

$$
\left(\begin{array}{l}
P_{x} \\
P_{y} \\
P_{z}
\end{array}\right)=\left(\begin{array}{lll}
\alpha_{x x} & \alpha_{x y} & \alpha_{x z} \\
\alpha_{y x} & \alpha_{y y} & \alpha_{y z} \\
\alpha_{z x} & \alpha_{z y} & \alpha_{z z}
\end{array}\right)\left(\begin{array}{l}
E_{x} \\
E_{y} \\
E_{z}
\end{array}\right)
$$

where ' $\alpha_{\mathrm{ij}}$ ' is the polarizability tensor.

The polarizability tensor can usefully be applied to scattering by fluids where not all molecules are in the same orientation. Hence, in liquid an averaged polarizability is measured. But the molecule contains 2 invariants, which do not depend on orientations. The mean value ' $\bar{\alpha}$ ' and the anisotropy ' $\gamma$ ' are the invariant properties of the polarizability tensor.

The mean value can be written as:

$$
\bar{\alpha}=\frac{1}{3}\left(\alpha_{\mathrm{xx}}+\alpha_{\mathrm{yy}}+\alpha_{\mathrm{zz}}\right)
$$

And the anisotropy as:

$$
\gamma^{2}=\frac{1}{2}\left[\left(\alpha_{x x}-\alpha_{y y}\right)^{2}+\left(\alpha_{y y}-\alpha_{z z}\right)^{2}+\left(\alpha_{z z}-\alpha_{x x}\right)^{2}+6\left(\alpha_{x y}^{2}+\alpha_{x z}^{2}+\alpha_{y z}^{2}\right)\right]
$$

Thus, the averaged scattering components of the polarization tensor can be expressed as:

and

$$
\bar{\alpha}_{\mathrm{ii}}^{2}=\bar{\alpha}_{\mathrm{xx}}^{2}=\bar{\alpha}_{\mathrm{yy}}^{2}=\bar{\alpha}_{\mathrm{zz}}^{2}=\frac{1}{45}\left(45 \bar{\alpha}^{2}+4 \gamma^{2}\right)
$$

$$
\bar{\alpha}_{\mathrm{ij}}^{2}=\bar{\alpha}_{\mathrm{xy}}^{2}=\bar{\alpha}_{\mathrm{xz}}^{2}=\bar{\alpha}_{\mathrm{yz}}^{2}=\frac{1}{15} \gamma^{2}
$$

The intensity of the scattered light is proportional to the square of the induced dipole moment. The following equations correspond to the isotropic and anisotropic components respectively.

$$
\begin{aligned}
& I_{z z}=k \alpha_{i i}^{2} E_{z}^{2} \\
& I_{z y}=k \alpha_{i j}^{2} E_{z}^{2}
\end{aligned}
$$




\subsubsection{Polarization measurements}

The polarization of scattered light relative to the input laser polarization has been used as a significant analytical tool. For a totally symmetric vibration, such as the symmetric stretch of $\mathrm{CCl}_{4}$, the Raman scattered light retains the polarization of the incident light. Such an experimental measurement is called parallel polarized measurement and the associated Raman band is said to be polarized.

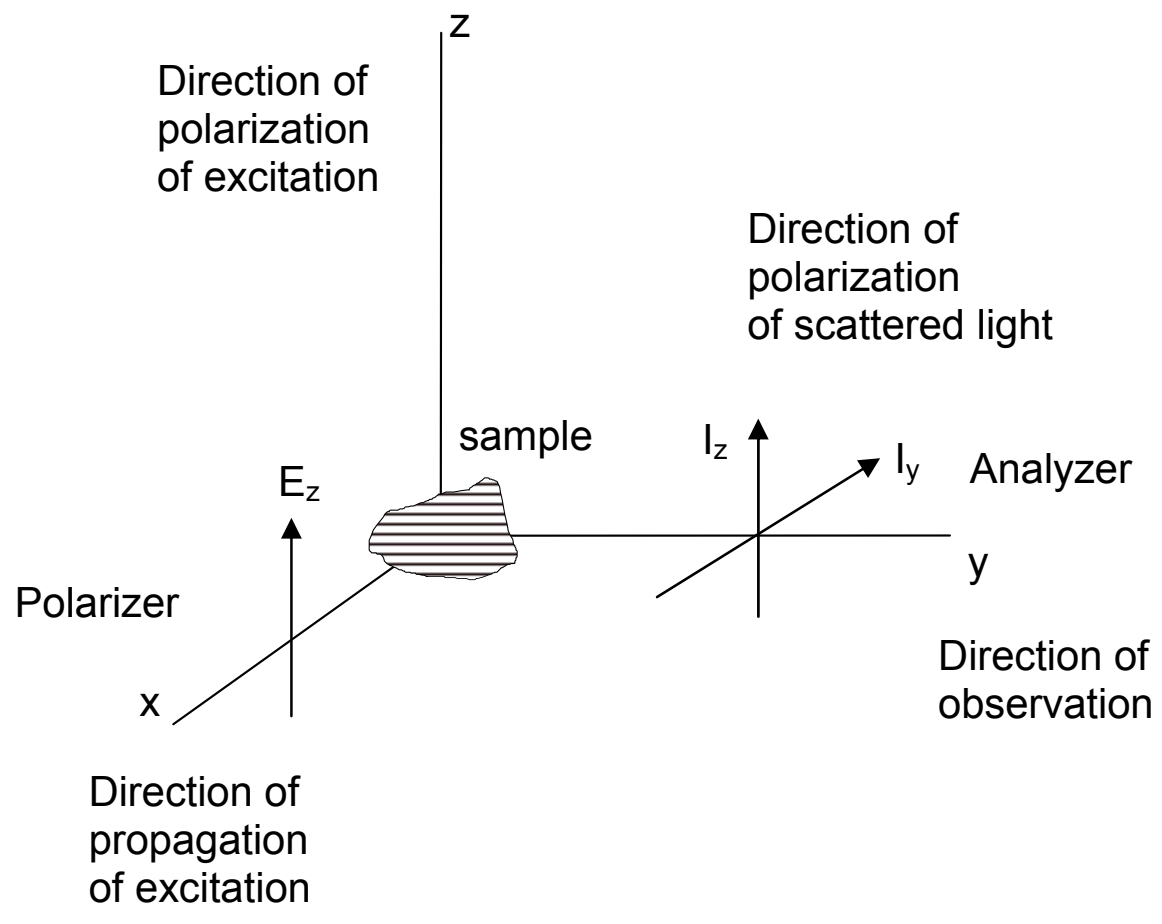

Figure 3. 4: Traditional Raman scattering geometry

Vibrations that are not totally symmetric exhibit significant scattering along the $\mathrm{x}$, $y$, and $z$ directions and are called depolarized bands. A traditional Raman $90^{\circ}$ scattering geometry is shown in Figure 3.4 with $x, y$ and $z$ as coordinates of the laboratory system of axes. The polarized bands or the parallel polarization can be observed in zz direction by keeping the polarizer and analyzer in z direction. Similarly, the perpendicular polarization measurements can also be done, where the scattered light is polarized perpendicular to the incident light ( $z x$ or $z y)$. The ratio of intensities of perpendicularly polarized band and parallel polarized band is called depolarization ratio ' $\rho$ '. The depolarization ratio is close to zero for 
symmetric vibrations or polarized bands and is close to 0.75 for asymmetric vibrations or depolarized bands. Both, the $90^{\circ}$ and $180^{\circ}$ (backscattering) sampling geometries can provide equivalent information about the polarization of Raman spectral features. The polarized (parallel polarized) band has both, isotropic and anisotropic contributions, whereas the depolarized band constitute only the anisotropic part. Expressions (3.19) and (3.20) show the relation of possible polarization measurements and their constituents.

$$
\begin{aligned}
& I_{Z Z}=I_{\text {isotropic }}+(4 / 3) I_{\text {anisotropic }} \\
& I_{Z Y}=I_{Z X}=I_{Y X}=I_{\text {anisotropic }}
\end{aligned}
$$

Ratio of equation 3.19 and 3.20 give the depolarization ratio ' $\rho$ '.

$$
\rho=\frac{I_{z y}}{I_{z z}}=\frac{\bar{\alpha}_{z y}^{2}}{\bar{\alpha}_{z z}^{2}}=\frac{1 / 15 \gamma^{2}}{1 / 45\left(45 \bar{\alpha}^{2}+4 \gamma^{2}\right)}=\frac{3 \gamma^{2}}{45 \alpha^{2}+4 \gamma^{2}}
$$




\subsubsection{Raman spectrometer}

We have employed a commercial T64000 triple system made by Jobin-Yvon. The three gratings of 1800 lines $/ \mathrm{mm}$ were operated in subtractive mode yielding a resolution of $1.5 \mathrm{~cm}^{-1}$. The Raman spectra were excited with an $\mathrm{Ar}^{+}$-laser (from Coherent Company) at $514.53 \mathrm{~nm}$. The scattered light was detected by charge coupled detector (CCD) which is cooled with liquid nitrogen. Figure 3.5 shows the Raman spectrometer T64000 (from Jobin-Yvon), which can be used in both micro and macro stage measurements.

All the micellar solutions were measured in macro stage, whereas the dry powder samples were measured in micro stage. We have the possibility of $90^{\circ}$ and $180^{\circ}$ geometry measurements, but we have done most of the measurements in $180^{\circ}$ geometry.

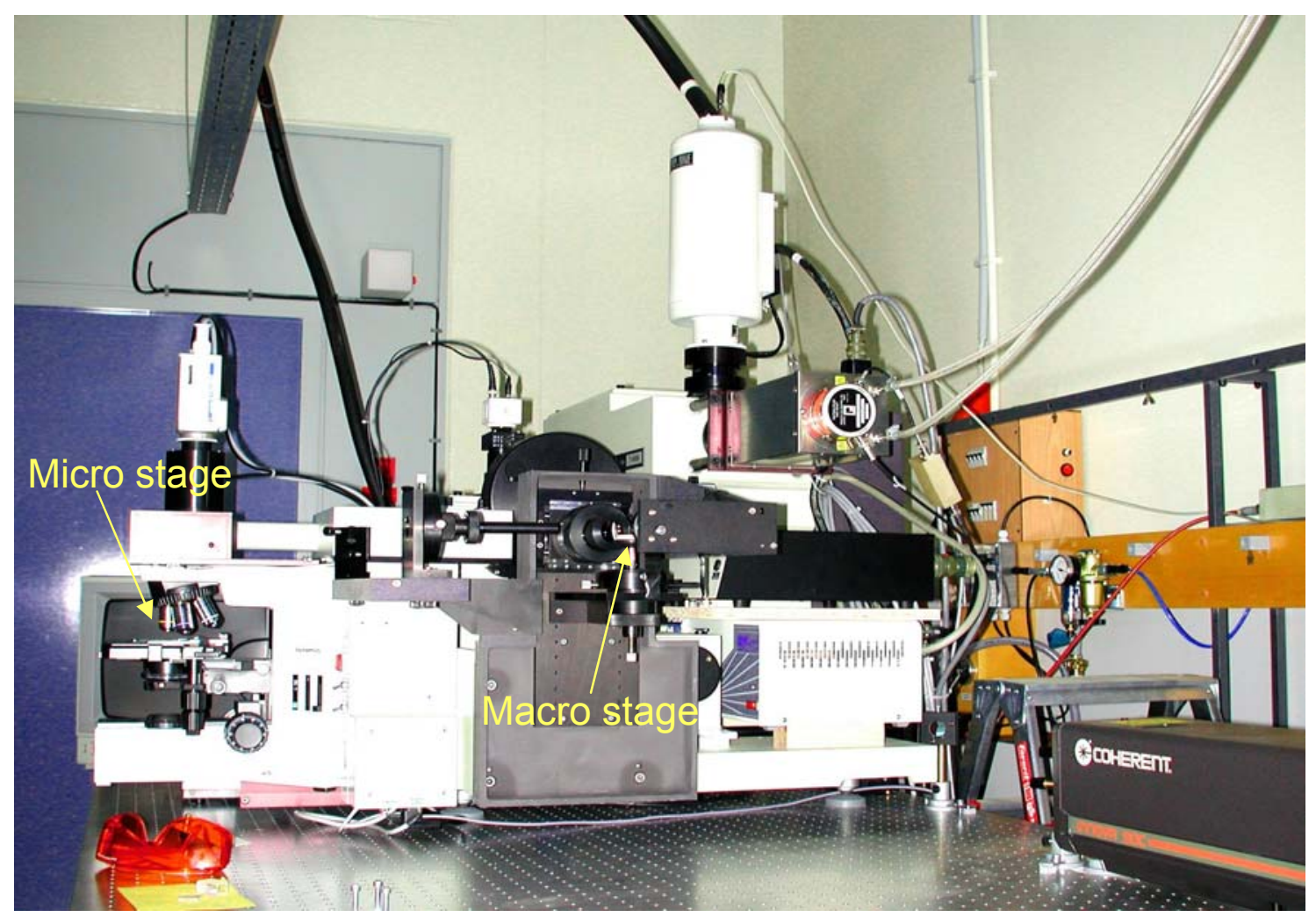

Figure 3. 5: Jobin-Yvon T64000 triple monochromator Raman spectrometer 
Raman spectra were recorded with the program Labspec. The commercial package Origin has been used to analyze the spectra. The raw data were baseline corrected with linear extrapolated bases before using them for fitting Gaussian or Lorentzian fit functions to extract the peak positions.

\subsubsection{Application of Raman spectroscopy}

Raman spectroscopy can be employed to understand the conformations of molecules in gas, liquid and solid phases. The pioneering work of Snyder et al. [Snyder_1960, Snyder_1961, Snyder_1963, Snyder_1967] on $n$-paraffins has been widely used by many authors to assign the molecular conformations in systems like, fatty acids, phospholipids and others surfactant assemblies [Lippert_1971, Yellin_1977, Gaber_1977, Levin_1981, Wong_1982, Akutsu_1981]. As it is mentioned in section 2.1.1, the hydrophobic part, or the alkyl chain of the surfactants makes the core of the micelles; Raman spectroscopy is a good tool to reveal the configuration/conformation of the alkyl chain. Peak frequency, full width half maxima and intensity height ratio of selected vibration bands give information on the configuration of the alkyl chain.

For the surfactant system tetradecyltrimethylammonium bromide (TTAB), both in aqueous micellar solution and powder sample, Raman spectra are presented in Figure 3.6. All measurements were measured with $\mathrm{zz}$ polarization unless otherwise mentioned. Spectral region (a), below $600 \mathrm{~cm}^{-1}$ is shown for the longitudinal accordion modes (LAM). Spectral region (b), from $875 \mathrm{~cm}^{-1}$ to 1500 $\mathrm{cm}^{-1}$ corresponds to the wagging, $\mathrm{C}-\mathrm{C}$ stretching, twisting and $\mathrm{CH}_{2} / \mathrm{CH}_{3}$ bending modes. And that of spectral region (c), from $2800 \mathrm{~cm}^{-1}$ to $3075 \mathrm{~cm}^{-1}$ corresponds to the $\mathrm{CH}_{2} / \mathrm{CH}_{3}$ stretching vibrations. A sketch of the few vibrational stretches is shown in Figure 3.7. A tentative assignment of the vibration bands of our interest is given in Table 3.1. Assignment of these bands is based on the literature [Kalyanasundaram_1976, Mantsch_1982, Wong_1983a, and Hattori_1999] for similar systems. 

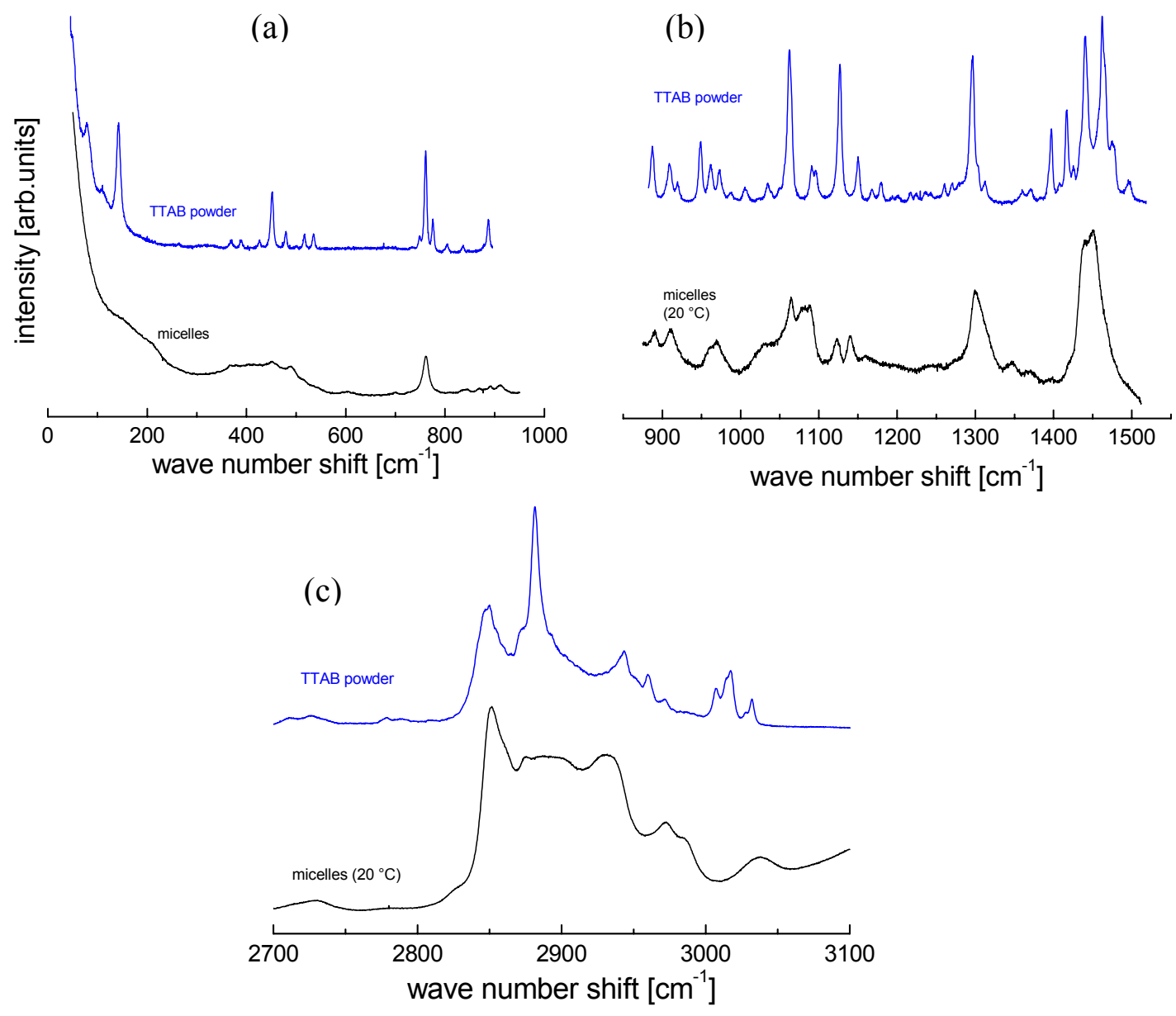

Figure 3. 6: Representative Raman spectra for powder TTAB and $0.4 \mathrm{M}$ aqueous micellar solution, (a) below $600 \mathrm{~cm}^{-1}$ longitudinal accordion modes, (b) C-C skeletal and $\mathrm{CH}_{2} / \mathrm{CH}_{3}$ bending modes, (c) $\mathrm{CH}_{2} / \mathrm{CH}_{3}$ stretching modes

The comparative study of micelles and powder samples of some surfactants were documented by Kalyanasundaram [Kalyanasundaram_1976]. The Raman spectra of aqueous micellar solutions are dominated by the broad bands whereas the powder samples are with sharp bands. The band analysis [Bartoli_1972], splitting of few vibrational modes [Casel_1982] have been studied and attributed to orientational fluctuations and intermolecular interactions. A detailed spectral interpretation of n-paraffins, moltan polyethylene and polymethylene chains have been done theoretically and found in good agreement with experimental values [Tasumi_1962, Snyder_1967, Boerio_1970]. 
Effect of odd and even numbered carbon atoms on vibrational frequencies and intermolecular interactions in case of crystalline $n$-paraffins is documented by Snyder [Snyder 1960, Snyder 1961].

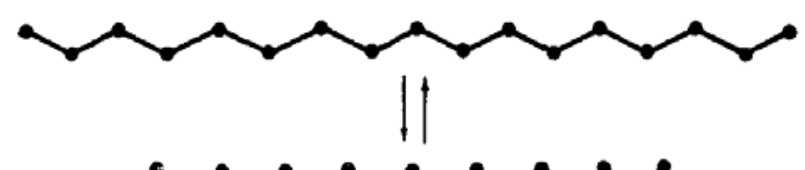

Longitudinal accordion modes
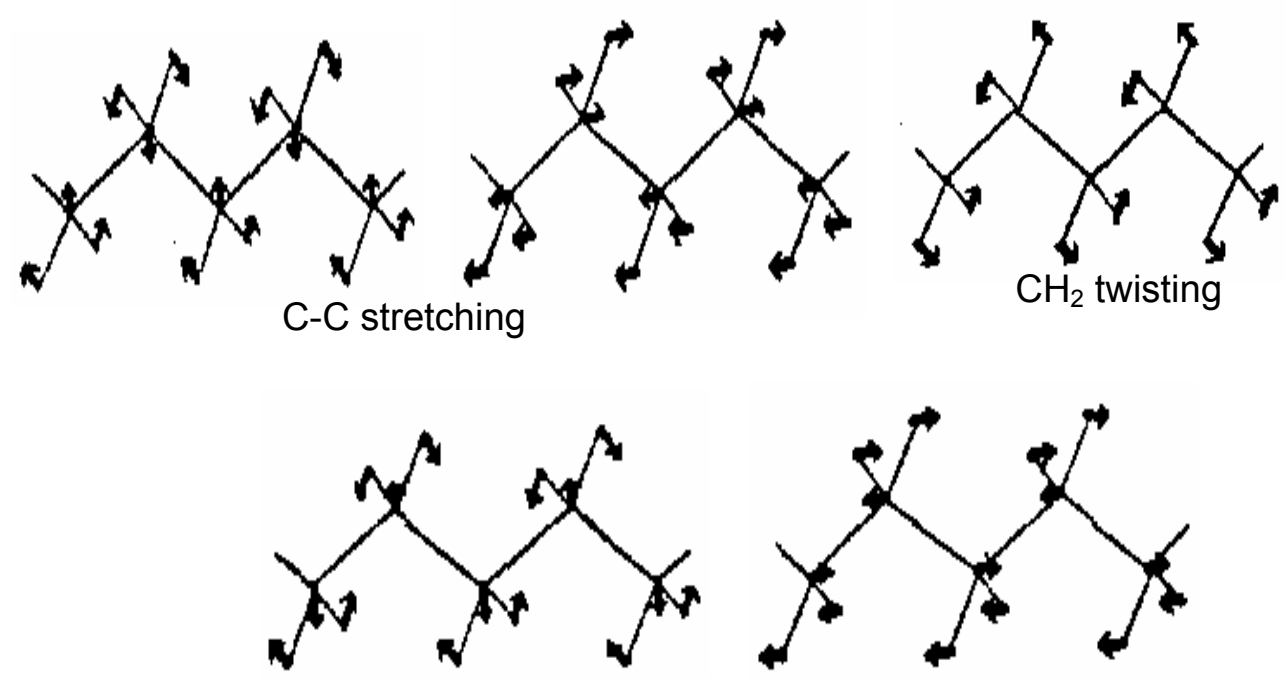

$\mathrm{CH}_{2}$ bending

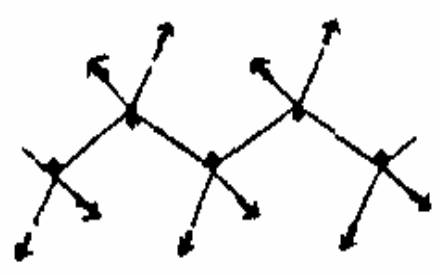

symmetric

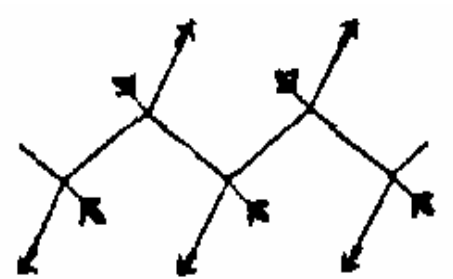

asymmetric

methylene $\mathrm{C}-\mathrm{H}$ stretching

Figure 3. 7: Schematics of the vibrations of alkane (adapted from Simanouti_1949, Schaufele_1967) 


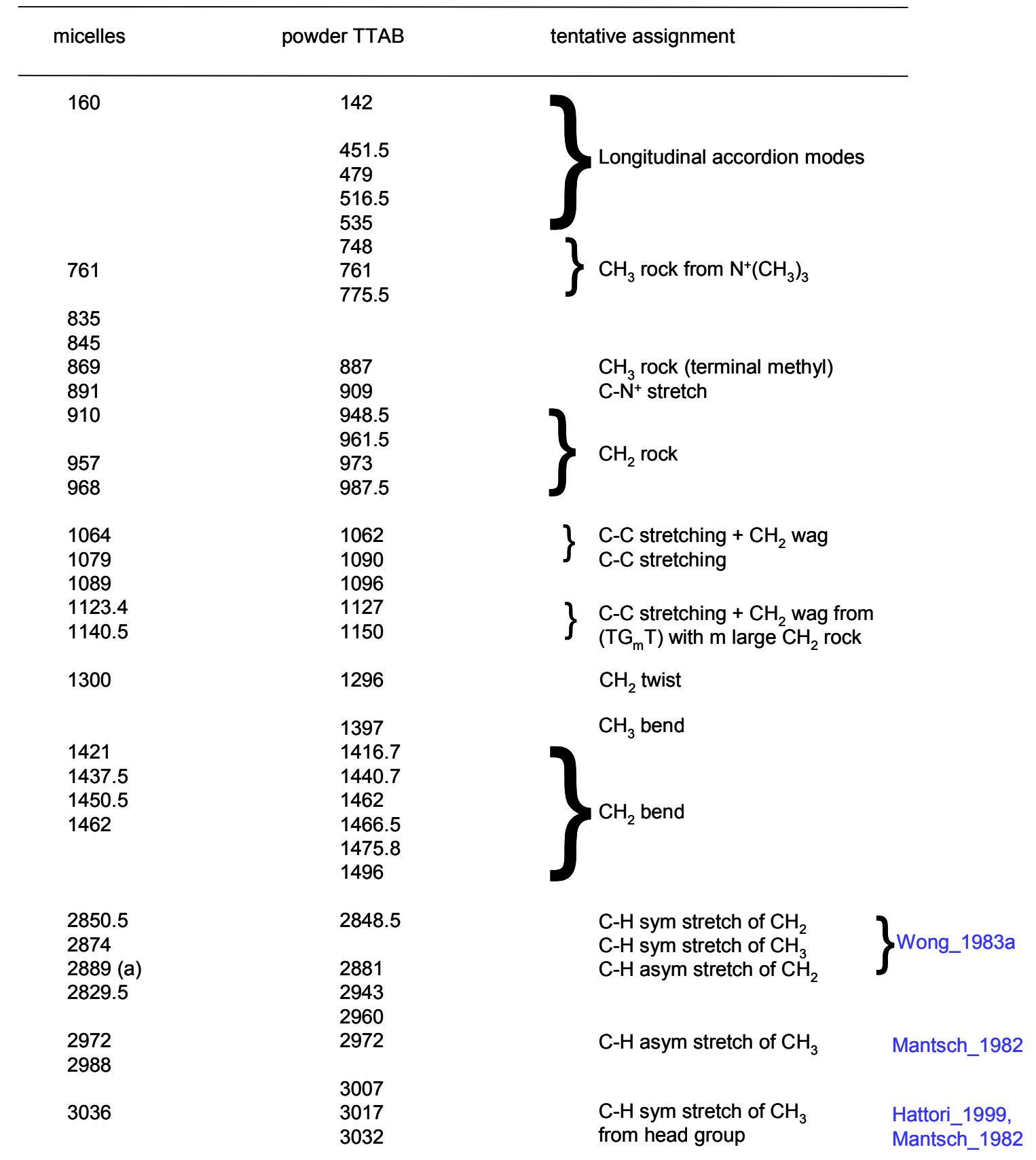

Table 3. 1: Tentative assignment for vibration bands of tetradecyltrimethylammonium bromide surfactant both in micelles and powder form, assignments are on the basis of literature. All the assignments taken from Kalyanasundaram_1976, unless otherwise referenced against the frequencies. (a) is measured in zy polarization 
P. T. T Wong [Wong_1983, Wong_1983a] has reported the structural changes of aqueous micellar solutions of sodium oleate employing IR and Raman spectroscopy with particular interest of influence of temperature and pressure. In such systems, with the influence of temperature or pressure, phase transition from micelles to coagel (solid like) phase was observed. The discontinuities in the peak frequency, intensity height ratio and full width half maxima (fwhm) are used to find the phase transition temperature or pressure. The change in peak frequency and fwhm strongly depends on the nature of the vibrational band, whether it is polarized or depolarized. The highly polarized bands are slightly narrower, whereas the depolarized bands are slightly broader. It is well accepted that the polarized and depolarized bands are affected by the conformation/configuration of the molecules. Width of the depolarized (or slightly polarized) bands are affected by the reorientational fluctuations, whereas the polarized (or isotropic) bands are unaffected by reorientational fluctuations [Bartoli_1972], but are strongly affected by interchain interactions [Abbott_1979, Kobayashi_1980]. Figure 3.8 shows the Raman spectra of TTAB, both, in polarized and depolarized measurements. Figure 3.8a presents the overall spectra for aqueous 0.4 M TTAB solutions, the spectral assignments are given in Table 3.1.
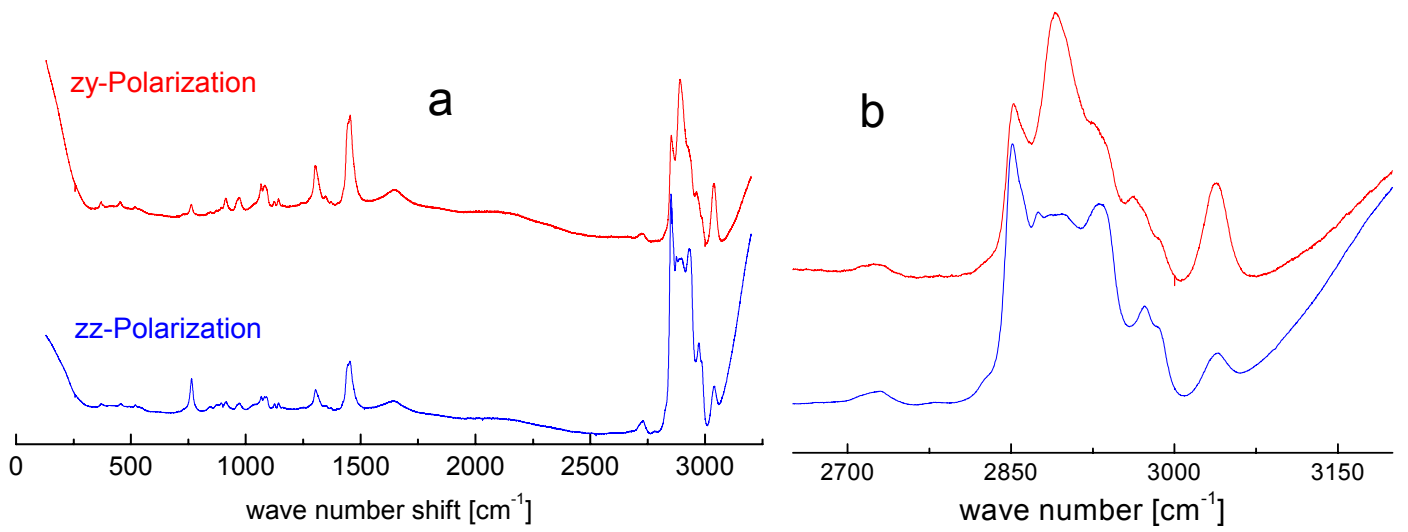

Figure 3. 8: Polarization measurements for $0.4 \mathrm{M}$ aqueous TTAB micellar solution at ambient conditions. Part "a" corresponds to the zz and zy polarized spectra and "b" the expanded part of "a" in $\mathrm{CH}_{2}$ stretching region for better view and spectrums are shifted vertically for clarity 
The spectral region around $2900 \mathrm{~cm}^{-1}$, which corresponds to the $\mathrm{CH}_{2}$ stretching vibrations, is shown separately in Figure $3.8 \mathrm{~b}$ for better clarity. The broad band in zz polarized spectra centered around $2889 \mathrm{~cm}^{-1}$ is the combination of asymmetric $\mathrm{CH}_{2}$ stretching and the Fermi resonance* bands [Snyder_1978, Snyder_1979, Snyder_1982]. This can be clearly demonstrated by measuring zy polarized spectra. If the asymmetric $\mathrm{CH}_{2}$ stretching band is not present in the broad band around $2889 \mathrm{~cm}^{-1}$, the relative intensity of the Fermi resonance band and the symmetric $\mathrm{CH}_{2}$ band should be same in $\mathrm{zz}$ and zy polarized spectra, which is clearly not the case as shown in Figure $3.8 \mathrm{~b}$. This shows that in zz polarization the asymmetric $\mathrm{CH}_{2}$ stretching band is present and overlapped with the Fermi bands. Asymmetric $\mathrm{CH}_{2}$ stretching band near $2889 \mathrm{~cm}^{-1}$ can be seen only in zy polarization.

According to equations 3.20 and 3.21 , the isotropic part of the $z z$ polarized spectra is presented in Figure 3.9 in comparison with the anisotropic part or the depolarized spectra, which gives an overview of the possible bands which can be affected by reorientational fluctuations.

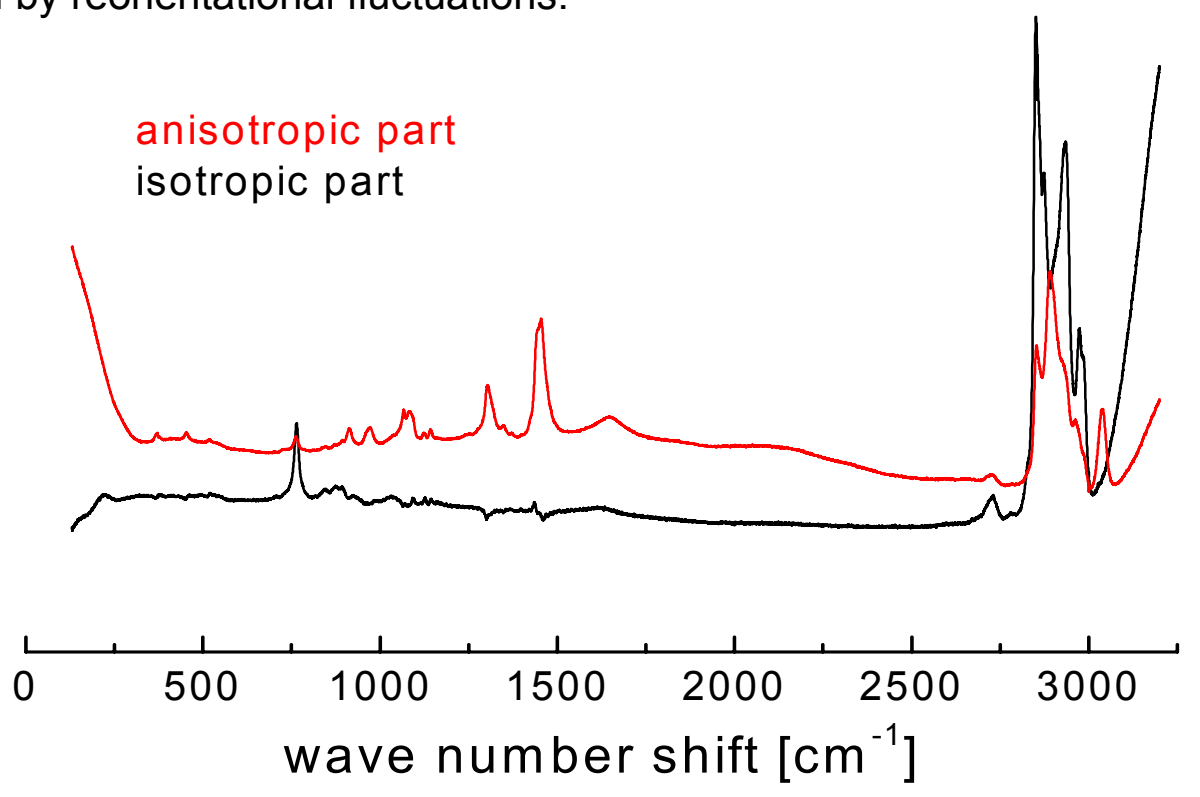

Figure 3. 9: Comparison of isotropic part of the zz polarzed spectra with depolarized spectra

* The fundamentals of unperturbed $\mathrm{C}-\mathrm{H}$ stretching $\left(\approx 2875 \mathrm{~cm}^{-1}\right)$ and $\mathrm{CH}_{2}$ bending overtones $(\approx 2$ $x 1441 \mathrm{~cm}^{-1}$ and $2 \times 1465 \mathrm{~cm}^{-1}$ ) have the almost same energy levels. The interactions or intercoupling between such an equal energy levels is termed as Fermi resonance [Liu_2006]. 


\subsection{Small Angle Neutron Scattering (SANS)}

Scattering experiments can be employed to determine the structure and the organization of particles in a homogeneous solution. The particles can be colloid or macromolecules like polymer or micelles with sizes ranges from angstroms to micrometers. The shape of the aggregate, spatial correlations and the spatial arrangement of the aggregates in the solution can be extracted. Light scattering, small angle X-ray scattering and neutron scattering techniques have been in use for such studies. The selection of the techniques depends on the properties of the sources, and the information one need on the matter (analyte). In some cases neutrons are preferred as they can penetrate deeper into the matter than the electrons and X-ray. Figure 3.10 depicts the very general schematic of the penetration of electrons, $\mathrm{X}$-rays and neutron into the matter [Figure adapted from Widjaja_2005].

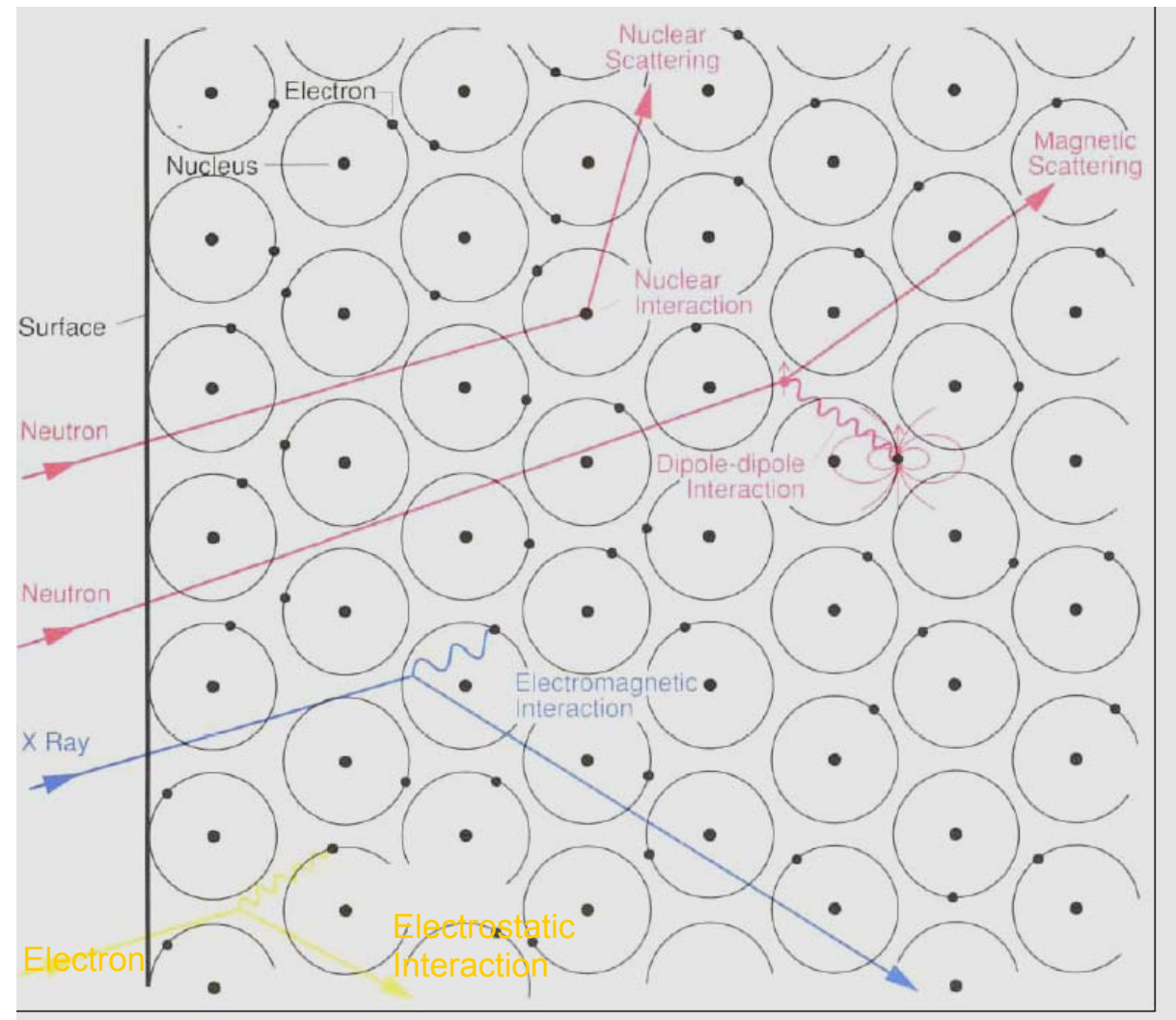

Figure 3. 10: Interaction of beams of neutrons, X-rays, and electrons with material (figure adapted from Widjaja_2005) 
Figure 3.10 shows, the interaction of beams of neutrons, X-rays, and electrons with material by different mechanisms. X-rays (blue) and electron beams (yellow) both interact with electrons in the material; with $\mathrm{X}$-rays the interaction is electromagnetic, whereas with an electron beam it is electrostatic. Both of these interactions are strong, and neither type of beam penetrates through matter very deeply. Neutrons (red) interact with atomic nuclei via the very short-range strong nuclear force and thus penetrate matter much more deeply than X-rays or electrons. If there are unpaired electrons in the material, neutrons may also interact by a second mechanism: a dipole-dipole interaction between the magnetic moment of the neutron and the magnetic moment of the unpaired electron.

\subsubsection{YUMO-SANS spectrometer}

In present work SANS technique has been employed to understand the structure of micellar solutions. The SANS measurements were performed at the small angle time of flight axially symmetric neutron scattering spectrometer YuMO equipped with a two detector system at IBR 2 fast pulsed reactor [Kuklin_2005], Dubna, Russia. The spectrometer is named in honor of Yurii M Ostanevich. A schematic view of the YuMO spectrometer is shown in Figure 3.11. The first detector was placed at a sample to detector distance of $5.28 \mathrm{~m}$ and the second one at $13.04 \mathrm{~m}$, thus yielding an overall range of wave vector transfer of $0.006<Q<0.3 \AA^{-1}$. The averaged scattering patterns were corrected for detector efficiency, absorption, solvent scattering and instrumental background. Vanadium (Vanadium sample) as internal standard and the $\mathrm{D}_{2} \mathrm{O}$ as an external standard were measured for the absolute calibration. Details of the SANS arrangement, absolute calibration, importance of the experiment's geometry and two detector system for SANS instrument, is available elsewhere [Ostanevich_1988, Maauouf_1994, Kuklin_2005]. Use of two detectors facilitate in widening of the momentum transfer dynamic range [Kuklin_2005]. 


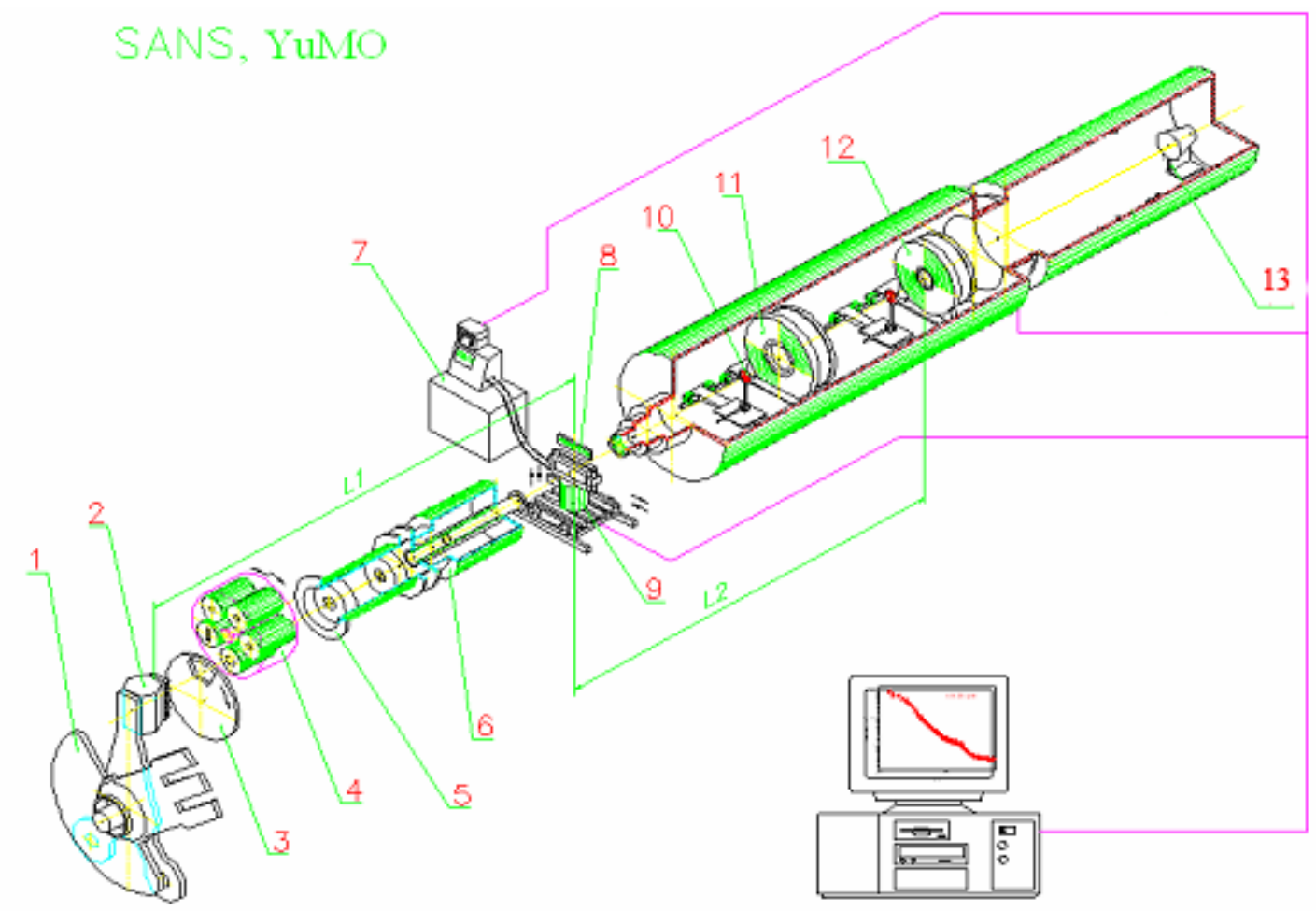

1. two reflectors, 2. zone of reactor with water moderator, 3. chopper, 4. first collimator, 5. neutron guide (vacuum tube), 6. second collimator, 7. thermostat, 8. table for sample, 10. Vn standard, 11 \& 12. scattering detector, 13. direct beam detector

Figure 3. 11: Schematic view of the YuMO spectrometer at IBR 2, Dubna, Russia

\subsubsection{Time of flight (TOF)}

The principle behind the TOF is the neutrons produced have different energy, and hence have different wavelength and travel with different velocity. This phenomenon hold good with the d Braglie's relation:

$$
\lambda=\mathrm{h} / \mathrm{mv}
$$

where ' $\lambda$ ' is wavelength, ' $m$ ' is the mass, ' $v$ ' is the velocity of the neutron and ' $h$ ' is plank constant.

For all neutrons produced at same time, the higher energy shorter wavelength neutrons travel faster and reach the sample and then detector faster than the 
lower energy longer wavelength slower neutrons. One can calculate the velocity and wavelength (energy) of the neutron by measuring the time of arrival of neutrons at the detector and the flight path. TOF is the wavelength sorted white beam technique of great use in diffraction and inelastic neutron scattering techniques. Using TOF method one can get the information on the structure of the analyte in solution or in solid.

\subsubsection{Theory of SANS}

The basic principle of SANS is measurement of the momentum transfer ' $Q$ '. The $Q$ can be calculated using the incident and scattered wave vectors $k_{i}$ and $k_{s}$ respectively, when a neutron beam passes through the sample as shown in the Figure 3.12.

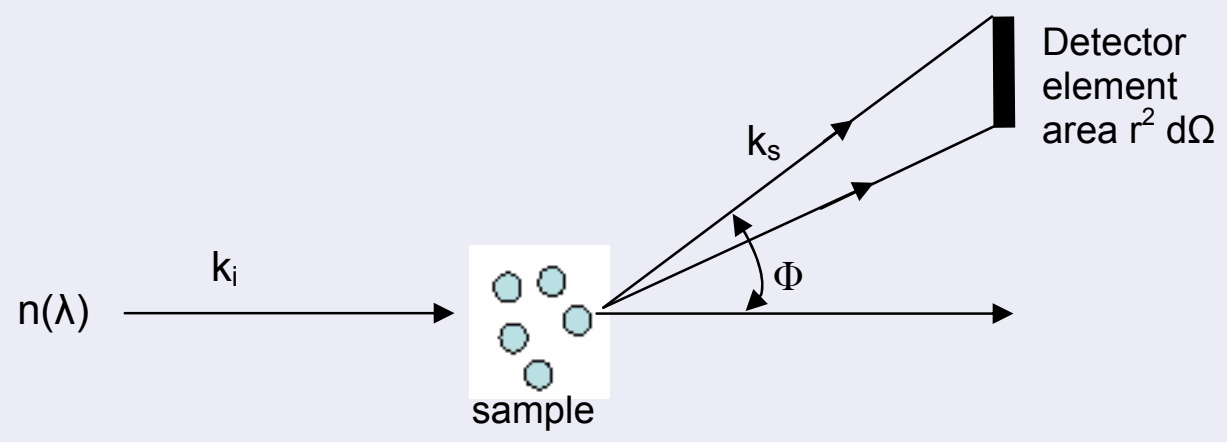

Figure 3. 12: Schematic representation of SANS scattering geometry, the terms used in the figures are described in the text

The $Q$ is defined as

$$
Q=k_{i}-k_{s}
$$

For elastic scattering

$$
\left|\mathrm{k}_{\mathrm{i}}\right|=\left|\mathrm{k}_{\mathrm{s}}\right|=2 \pi / \lambda
$$

Consequently, the modulus of the $Q$ vector turns out to be:

where ' $\Phi$ ' is the scattering angle

$$
|\mathrm{Q}|=\frac{4 \pi}{\lambda} \sin \left(\frac{\Phi}{2}\right)
$$


SANS is being extensively used for the study of soft/condensed matters. SANS has high sensitivity in the size range of $1-100 \mathrm{~nm}$ and thus can be used to probe complex systems containing individual particles, aggregates and large agglomerates with distinct length scales. To probe the above length scales one requires data in a wide $Q(4 \pi / \lambda \sin (\Phi / 2))$ ranges of $0.006-0.3 \AA^{-1}$. This $Q$ range can be achieved with neutrons of wavelength ranging from $0.7-10 \AA$.

Another important aspect of neutron scattering measurements is that of scattering contrast between the particles in the solution and the solvent, which is generally written as

$$
\Delta \rho=\rho_{\mathrm{p}}-\rho_{\mathrm{s}}
$$

where ' $\rho_{p}$ ' and ' $\rho_{s}$ ' are scattering length densities (sld) of the particles and the solvent. The sld is defined as

$$
\rho=\frac{\sum_{i=1}^{n} b_{i}}{V}
$$

where ' $\mathrm{V}$ ' is the volume containing $\mathrm{n}$ atoms, and ' $\mathrm{b}_{\mathrm{i}}$ ' is the scattering length of the $\mathrm{i}^{\text {th }}$ atom in the volume ' $\mathrm{V}$ '.

It is worth to note that, even the isotopes of the same element have different scattering lengths. This aspect is been in use to study the structure of surfactant aggregation such as micelles, polymers, phospholipids etc.

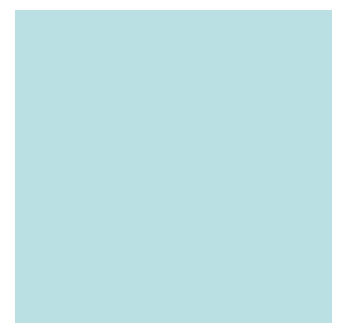

box 1

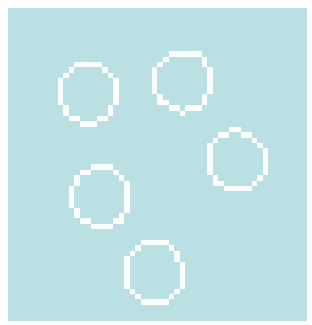

box 2

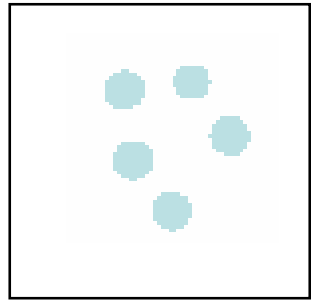

box 3

Figure 3. 13: Schematic illustration of isotopic effect in SANS study

Figure 3.13 shows schematic illustration of isotopic effect in SANS study. The scattering length densities of light water $\left(\mathrm{H}_{2} \mathrm{O}\right)$ and heavy water $\left(\mathrm{D}_{2} \mathrm{O}\right)$ are $-0.56 \mathrm{x}$ 
$10^{10} \mathrm{~cm}^{-2}$ and $6.38 \times 10^{10} \mathrm{~cm}^{-2}$ respectively. For the sample and the solvent of the same atoms, for example $\mathrm{H}$ rich atoms in surfactant and the $\mathrm{H}_{2} \mathrm{O}$ as solvent there is no difference in scattering length densities and therefore no structural informations as shown in first box, the second and third box illustrates the only head group deuterated surfactant in $\mathrm{H}_{2} \mathrm{O}$ as solvent, and normal surfactant in $\mathrm{D}_{2} \mathrm{O}$ as solvent respectively, which yield the structural information of the surfactant aggregations.

\section{Scattering cross sections}

The scattering scross sections is also termed as differential scattering cross section given by the mathematical form

$$
\frac{d \Sigma}{d \Omega}(Q) \equiv \frac{1 d \sigma}{V} \frac{d}{d}(Q)
$$

And it is also denoted as the normalized intensity ' $(Q)$ '.

The scattered intensity, for a system of single particles like micelles is given by the relation;

$$
\mathrm{I}(\mathrm{Q})=\left(\rho-\rho_{\mathrm{s}}\right)^{2} \mathrm{~V}_{\mathrm{m}}^{2} \mathrm{P}(\mathrm{Q})
$$

where ' $\rho$ ' and ' $\rho_{s}$ ' are the scattering length density of the micelles and the solvent, respectively, ' $V_{m}$ ' is the apparent volume of the micelle and ' $P(Q)$ ' is the orientational average of the form factor for a single micelle.

The form factor ' $P(Q)$ ' for spherical monodisperse objects is given by [Gorski_1994]

$$
P(Q)=\int_{\mathbf{V}} \mathbf{e}^{\mathbf{i Q} \cdot \mathbf{r}} \mathbf{d r}=\left\{\frac{[\sin (\mathrm{QR})-(\mathrm{QR}) \cos (\mathrm{QR})]}{(\mathrm{QR})^{3}}\right\}^{2}
$$

For the homogeneous cylindrical objects, the form factor is given by the relation [Hoffmann_1983]

$$
P(Q)=\int_{0}^{\pi / 2}\left\{\frac{\sin [Q(L / 2) \cos \beta]}{Q(L / 2) \cos \beta} \frac{2 J_{1}(Q R \sin \beta)}{Q R \sin \beta}\right\}^{2} \sin \beta d \beta
$$


where ' $R$ ' stands for the radius of the spheres or of the cylinder, ' $L$ ' stands for length of the cylinder, ' $J$ ' is the Bessel function of first order and ' $\beta$ ' is the angle between the symmetry axis of the micelle and the vector of momentum transfer.

The scattering cross section for assembly of interacting particles equation 3.29 extends to;

$$
\mathrm{I}(\mathrm{Q})=\mathrm{n}\left(\rho-\rho_{\mathrm{s}}\right)^{2} \mathrm{~V}_{\mathrm{m}}^{2} \mathrm{P}(\mathrm{Q}) \mathrm{S}(\mathrm{Q})
$$

where ' $S(Q)$ ' is the interparticle structure factor, scattering of spatial arrangement of N particles can be described as [Chen_1986]

$$
S(Q)=\frac{1}{N}\left\langle\sum_{i=1}^{N} \sum_{j=1}^{N} \exp \left[i Q\left(r_{i}-r_{j}\right)\right]\right\rangle
$$

where ' $r_{i}-r_{j}$ ' is the position of the scattering particles $i$ and $j$.

Structure factor is an important parameter in the scattering experiments since, it can be directly measured and the structural information can be determined with fitting the experimental data with the theoretical models. Hayter and Penfold [Hayter_1981] have developed an analytical method to determine the structure factor for the monodisperse macroions by solving the Ornstein-Zernike equation [Ornstein_1914]. It takes into account the mutual (screened) Coulomb interactions between the particles in solutions and assumes that the particles are monodisperse spheres.

\section{Data analysis}

Combining the structure factor obtained from the Hayter-Penfold model with the form factor of the particles, a scattering law is obtained that can directly be compared with the experimental data. Figure 3.14 is shown for SANS scattering profile for $25 \mathrm{mM}$ aqueous $\left(\mathrm{D}_{2} \mathrm{O}\right)$ TTAB solution in comparison with Hayter and Penfold model fitting. Even if the neutron small angle data are dominated by the maximum of the structure factor, the form factor and hence, the geometrical parameters of the micelles can thus be determined. The computer program FISH 
[Heenan] have been used to fit the SANS-data to this model. All the geometrical parameters of the fittings will be discussed in Chapter 4 'results and discussions'.

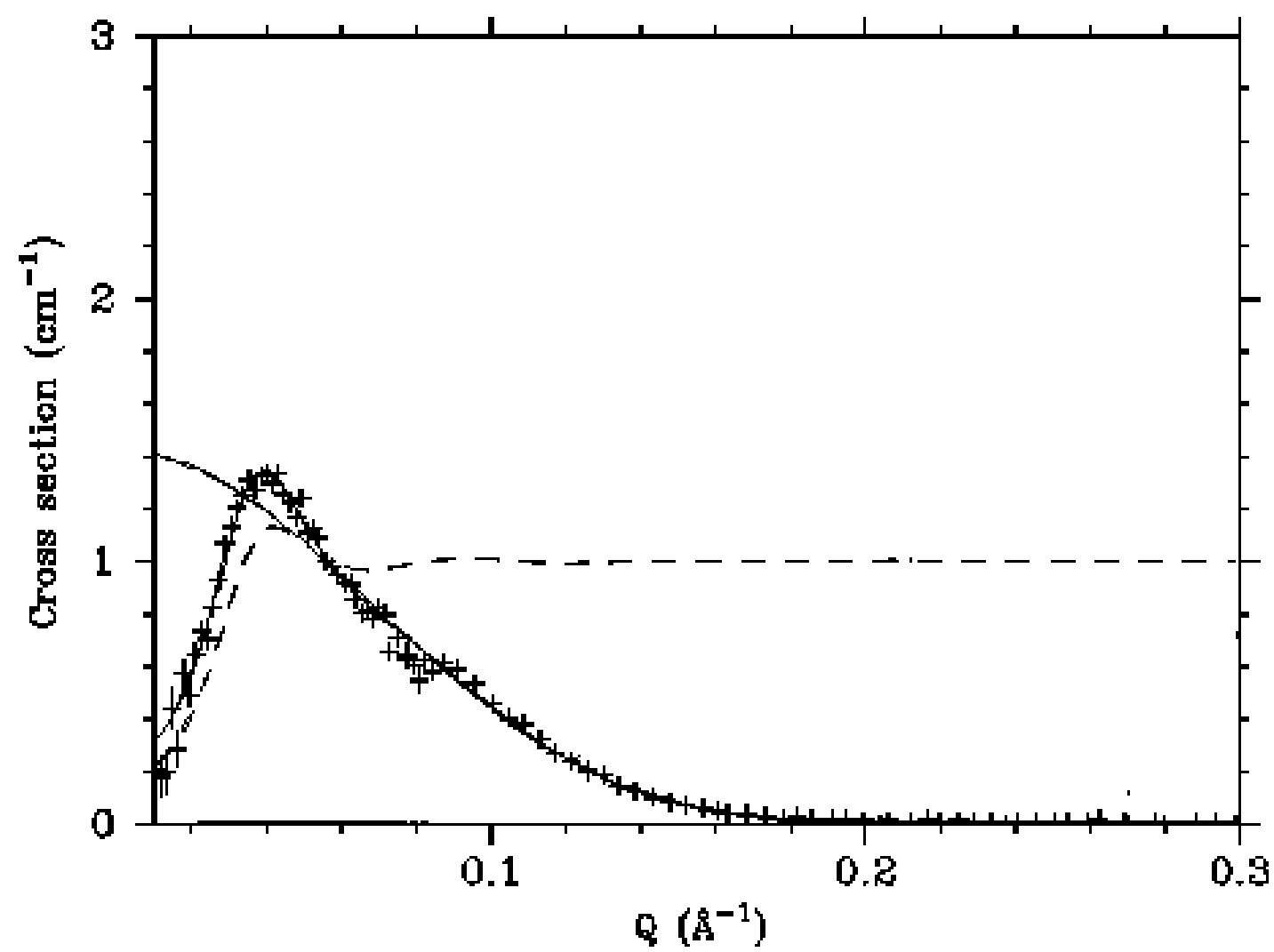

Figure 3. 14: SANS intensity profile of $25 \mathrm{mM}$ TTAB in $\mathrm{D}_{2} \mathrm{O},(+)$ are experimental, the solid line for form factor and the dashed line is for structure factor from the fits of Hayter-Penfold model

\subsubsection{Application of SANS}

SANS is being used to study the structural understanding of micellar solutions of ionic and non-ionic surfactant systems by many authors [Bendedouch_1983, Chen_1986 Gorski_1994, Bergström_1999]. Influence of temperature [Gorski_2001], pressure [Gorski_1999, Bossev_2001], added salt [Eckold_2001, Aswal_2000, Aswal_2003], alkyl chain length [Aswal_2002], and presence of multiple head groups [Haldar_2001] on the structure of micellar solutions of some surfactant have been done. It's noted that the aggregation number decreases with increase of temperature. The phase transition from micellar phase to solid 
like phase was observed with increase of pressure. Added electrolyte, which reduces the intermicellar repulsions in ionic micelles increases the micellar length, and hence the aggregation number. Presence of multiple head group in a surfactant decreases the aggregation number, because of the geometrical constraints. Increase of alkyl chain length of a surfactant (above C14) produces some intermediate phases (generally, metastable gel phase) before they transfer from micelles to solid-like phase.

\subsection{Pressure cells}

A specially designed optical high pressure cell was employed for the Raman spectroscopic study is shown in Figure 3.15a. Pressure of up to 2700 bar and temperature of about $300{ }^{\circ} \mathrm{C}$ can be achieved with such setup. Its basic design is described by M Buback [Buback_1994].

(a)

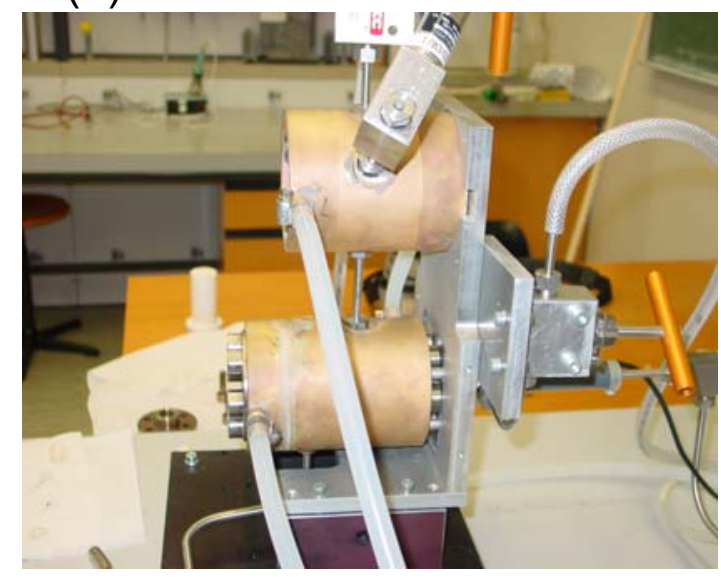

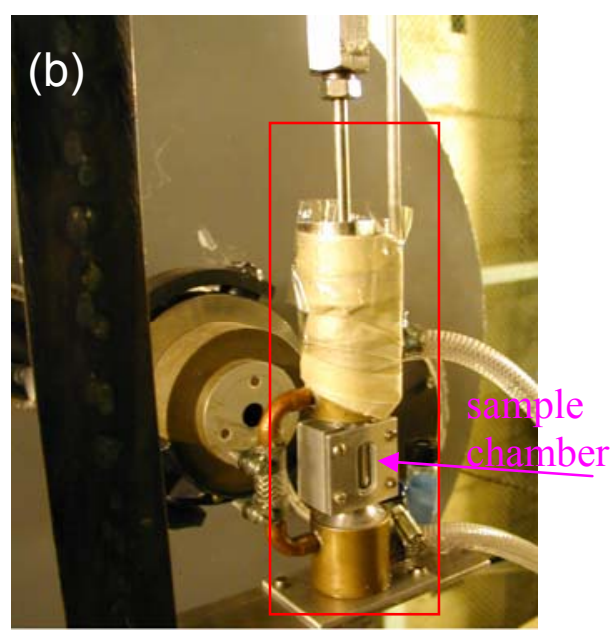

Figure 3. 15: (a) Pressure cell used for Raman spectra measurements, (b) Pressure cell used for SANS measurements

The pressure cell setup is made up of two cells (generally called twin cell). With the use of twin cell one can separate the sample (surfactant) solution from the hydraulic pressure generator. The pre cell (lower part) of this setup is connected to the screw press and is operated with normal water. It is separated from the 
sample cell (upper part) by moveable bellows. Hence, the sample cell can be filled independently with the solution under consideration. The twin cell is made from stainless heat resisting steel (Nimonic 90, Inconel 718) and it has sapphire windows for the laser to pass through the sample. The pressure was applied by means of hand pump and the pressure at the sample window was measured with a pressure gauge. The whole setup allows one to vary the path length. In present case, the usual path length chosen was $10 \mathrm{~mm}$. Using the heating jacket, the temperature of the sample was controlled within $0.1^{\circ} \mathrm{C}$. Lauda thermostat was used with silicon oil as heat transferring medium.

For the purpose of neutron scattering measurements a high pressure cell made from aluminium/titanium alloy of high tensile strength was used (shown in fig. $3.15 \mathrm{~b}$, red box), which was kindly supplied by Prof. R Winter from the University of Dortmund [Winter_1989]. The inner diameter of the cylindrical pressure cell was $5 \mathrm{~mm}$. The pressure was applied by means of hand pump. Temperature of the cell was controlled within $0.1{ }^{\circ} \mathrm{C}$, by circulating silicon oil using Lauda thermostat.

\section{Quartz cuvettes}

For measurement of Raman spectra of micellar solutions at ambient pressure, double walled quartz cuvettes of volume $1.4 \mathrm{ml}$ (from Hellma) were used. Temperature of the sample was controlled by a thermostat (circulating water system) with an accuracy of $0.1^{\circ} \mathrm{C}$.

In the case of SANS experiments micellar solution is placed in standard quartz cuvette (from Hellma) with the thickness of $2 \mathrm{~mm}$ (volume $2.6 \mathrm{ml}$ ). A special sample holder is available to place 14 cuvettes and the same can be fixed in a thermostat and the temperature of the sample can be maintained with an accuracy of $0.1^{\circ} \mathrm{C}$. 


\subsection{Surfactants}

Alkyltrimethylammonium Bromide surfactants are used in this study. Alkyl chain with even numbered carbon atoms from $\mathrm{C} 12$ to $\mathrm{C} 18$ is selected and they are known as dodecyl, tetradecyl, hexadeyl and octadecyl trimethylammonium bromides respectively. For the simpler notations they are also written as DTAB, TTAB, HTAB and OTAB. For a benchmark study, tetradecyltrimethylammonium Bromide (C14, TTAB, MyTAB, TTABr) has been used. A schematic view of the molecular structure of TTAB is shown in Figure 3.16.

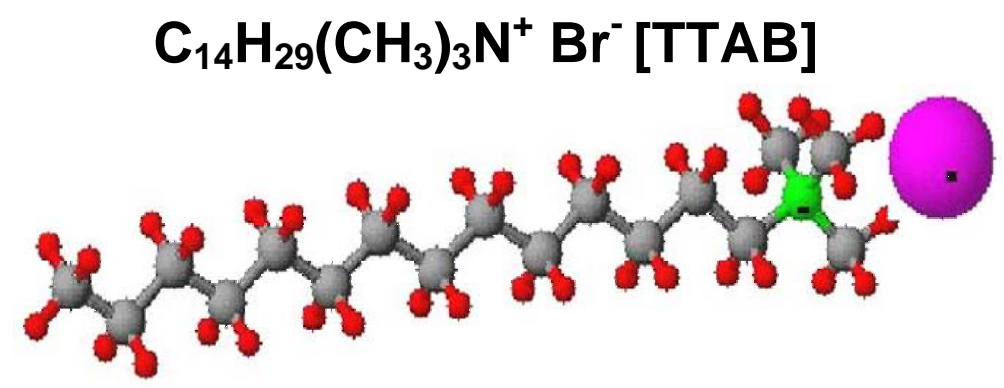

Figure 3. 16: Schematic representation of TTAB surfactant molecule, red, dark, green and magenta colored balls represent hydrogen, carbon, nitrogen and bromide atoms respectively

Surfactants DTAB and TTAB (grade 99\% each) were purchased from SigmaAldrich Company, whereas HTAB (grade 99\%) and OTAB (grade 97\%) were purchased from Fluka Bio Chemika/Chemika Company. Electrolyte, $\mathrm{NaBr}$ (grade 99\%) was purchased from Fluka Chemika Company.

All the above mentioned chemicals were used without further purification. For Raman spectrum and SANS measurements, solutions were prepared in double distilled water $\left(\mathrm{H}_{2} \mathrm{O}\right)$ and deuterated water $\left(\mathrm{D}_{2} \mathrm{O}, 99.8 \%\right)$ from "Isotop", Moscow respectively. 


\section{Results and Discussions}

The first part of this chapter starts with the studies on a surfactant tetradecyltrimethylammonium bromide (TTAB) molecule which has been chosen as a model system (surfactant) to understand the structure and dynamics in micellar solution and associated phases under different thermodynamic conditions.

\subsection{Critical micelle concentration (cmc) determination}

Figure 4.1 shows the plot of conductance ' $\kappa$ ' against TTAB in water at $25^{\circ} \mathrm{C}$. The break in the initial slope is observed at $3.8 \mathrm{mM}$ with increase in surfactant concentration. Above this concentration the slope changes because of the reduction in the degree of dissociation of the surfactant molecules. The break point at $3.8 \mathrm{mM}$ TTAB concentration gives the $\mathrm{cmc}$ of the TTAB solution in water at ambient conditions (at $25^{\circ} \mathrm{C}$ and atmospheric pressure). This value is in good agreement with the literature [Domínguez_1997, Zielinski_1987, Stodghill_2004].

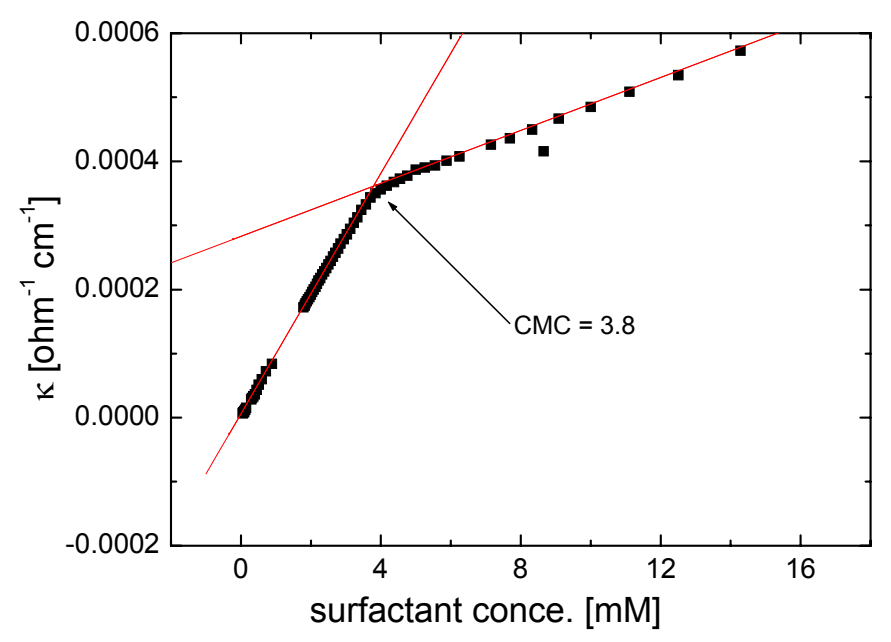

Figure 4. 1: Critical micelle concentration $(\mathrm{cmc})$ of TTAB determination by conductivity measurement 


\subsection{Aggregation behaviors at ambient conditions}

Aggregation behavior of aqueous micellar solution of TTAB at ambient conditions can be investigated as follows. Raman spectra of $0.4 \mathrm{M}$ aqueous TTAB solution are presented in Figure 4.2. Various spectral windows provide information on the behavior of both, hydrophilic (head group) and hydrophobic (alkyl chain) part of the micelles. Figure $4.2 \mathrm{a}$ provides information on the longitudinal accordion modes (LAM) which covers the frequency regime of $50 \mathrm{~cm}^{-1}$ to $950 \mathrm{~cm}^{-1}$. Figure 4.2b provides information on $\mathrm{C}-\mathrm{H}$ bending from $\mathrm{CH}_{2} / \mathrm{CH}_{3}$ groups, $\mathrm{CH}_{2}$ twisting and $\mathrm{C}-\mathrm{C}$ stretching in the frequency regime of $900 \mathrm{~cm}^{-1}$ to $1500 \mathrm{~cm}^{-1}$. Figure $4.2 \mathrm{c}$ presents $\mathrm{C}-\mathrm{H}$ stretching vibrations of methylene $\left(\mathrm{CH}_{2}\right)$ and methyl $\left(\mathrm{CH}_{3}\right)$ groups in the frequency regime of $2650 \mathrm{~cm}^{-1}$ to $3100 \mathrm{~cm}^{-1}$. The spectral regime in Figure $4.2 \mathrm{~b}$ constitutes the conformations of the alkyl chain (trans and gauche conformations). Spectral features are already discussed in Chapter 3 and the tentative assignments for both micellar solution and powder samples are tabulated in Table 3.1. It is observed that in micellar solutions the vibration bands are broad and most of them have gained higher wave number in comparison to the powder TTAB. Such broad vibrational bands are characteristic of n-paraffins in their liquid state [Snyder_1967, Barnes_1972].

(a)

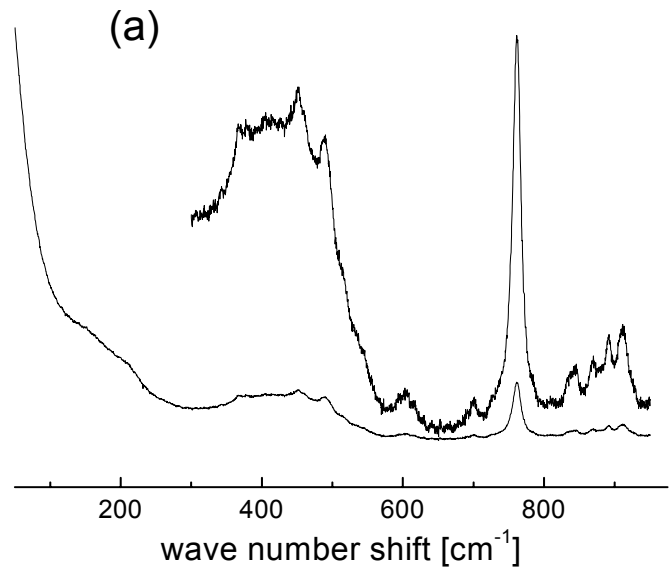

(b)

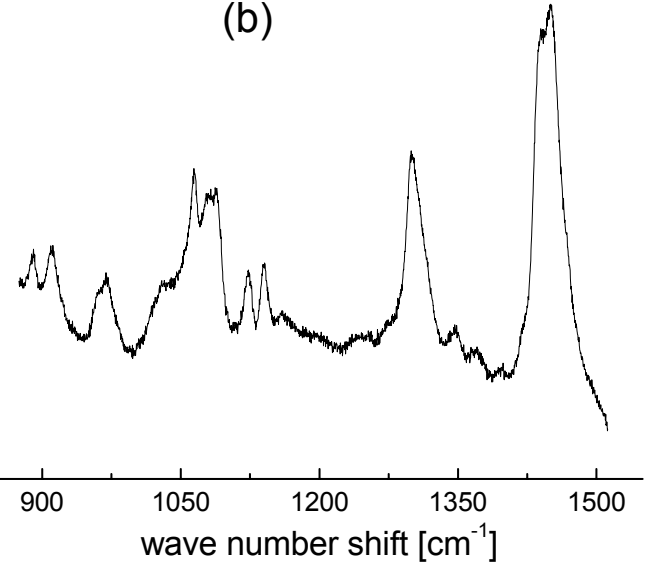

Figure 4. 2: Raman spectrum of $0.4 \mathrm{M}$ TTAB in water, (a) longitudinal accordion modes (below $300 \mathrm{~cm}^{-1}$ ). The intensity of the spectral region $300 \mathrm{~cm}^{-1}$ to $950 \mathrm{~cm}^{-1}$ is enhanced by a factor of 3 for better peak resolution,(b) $850 \mathrm{~cm}^{-1}$ to $1500 \mathrm{~cm}^{-1}$ corresponds to skeletal deformations 
(c)

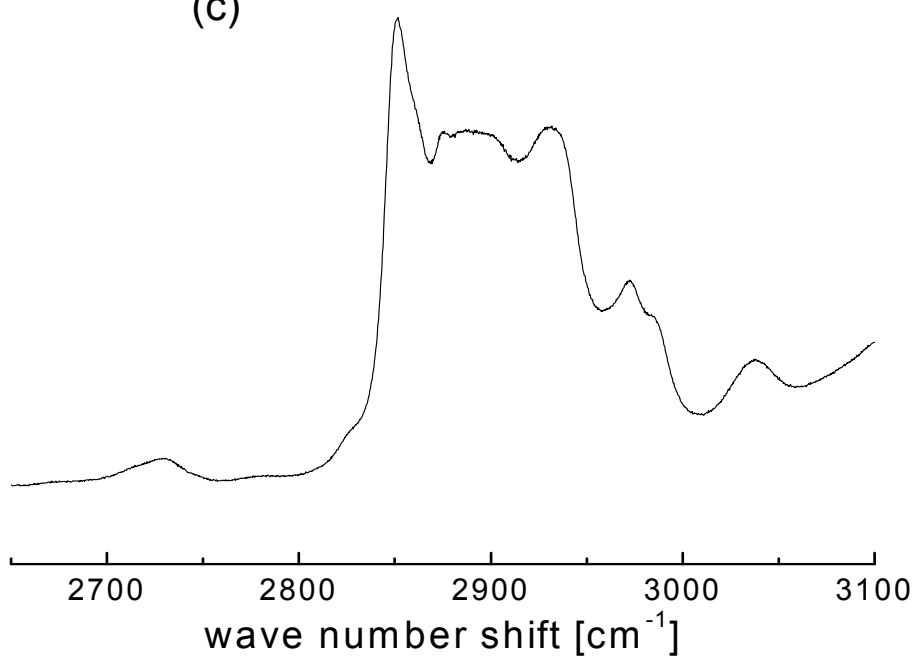

Figure 4.2c: Raman spectrum of $0.4 \mathrm{M}$ TTAB in water showing spectral regime 2650 $\mathrm{cm}^{-1}$ to $3150 \mathrm{~cm}^{-1}$ corresponds for the $\mathrm{C}-\mathrm{H}$ stretching

In the liquid state paraffins chains exist with many gauche conformations and hence, kinks in their alkyl chains. Raman spectrum of the micellar solutions reveals that the inner part of the micelles (alkyl chain of the surfactants), so called core of the micelle is essentially liquid paraffin like with many gauche conformations. The presence of gauche conformation is mainly indicated by the peak near $1090 \mathrm{~cm}^{-1}$ and $1140 \mathrm{~cm}^{-1}$ and that of trans peaks near $1064 \mathrm{~cm}^{-1}$ and $1123 \mathrm{~cm}^{-1}$. From the above Raman spectra one can obtain qualitative information that at ambient conditions alkyl chains of the micelles have both, trans and gauche conformations.

Having in mind this qualitative information about the micelles the quantitative information has been extracted from small angle neutron scattering (SANS) results. SANS can yield aggregation number, size, shape and charge of the micelles. Surfactant concentrations between $25 \mathrm{mM}$ to $670 \mathrm{mM}$ have been used to extract the geometrical parameters of the micelles at ambient conditions. For surfactant concentration of $25 \mathrm{mM}$, the SANS intensity profile is shown in Figure 4.3. Figure 4.4 presents the representative SANS intensity profiles at different TTAB concentrations. 


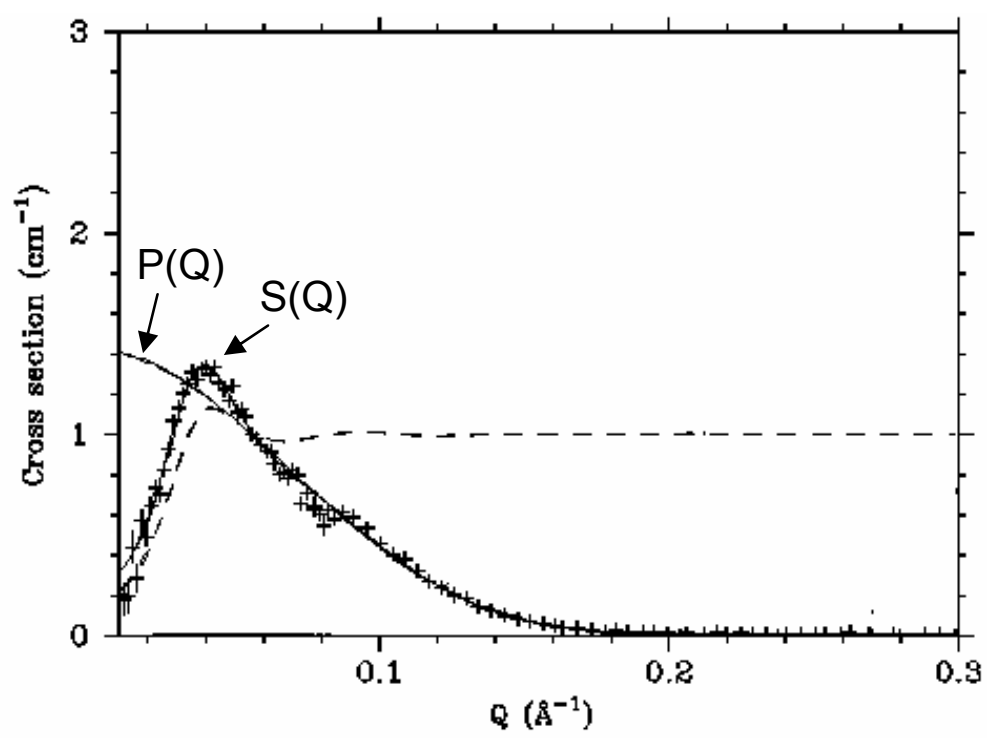

Figure 4. 3: SANS intensity profile of $25 \mathrm{mM} \mathrm{TTAB}$ in $\mathrm{D}_{2} \mathrm{O},\left(_{+}\right)$are experimental, the solid line for form factor and the dashed line is for structure factor from the fits of Hayter-Penfold model

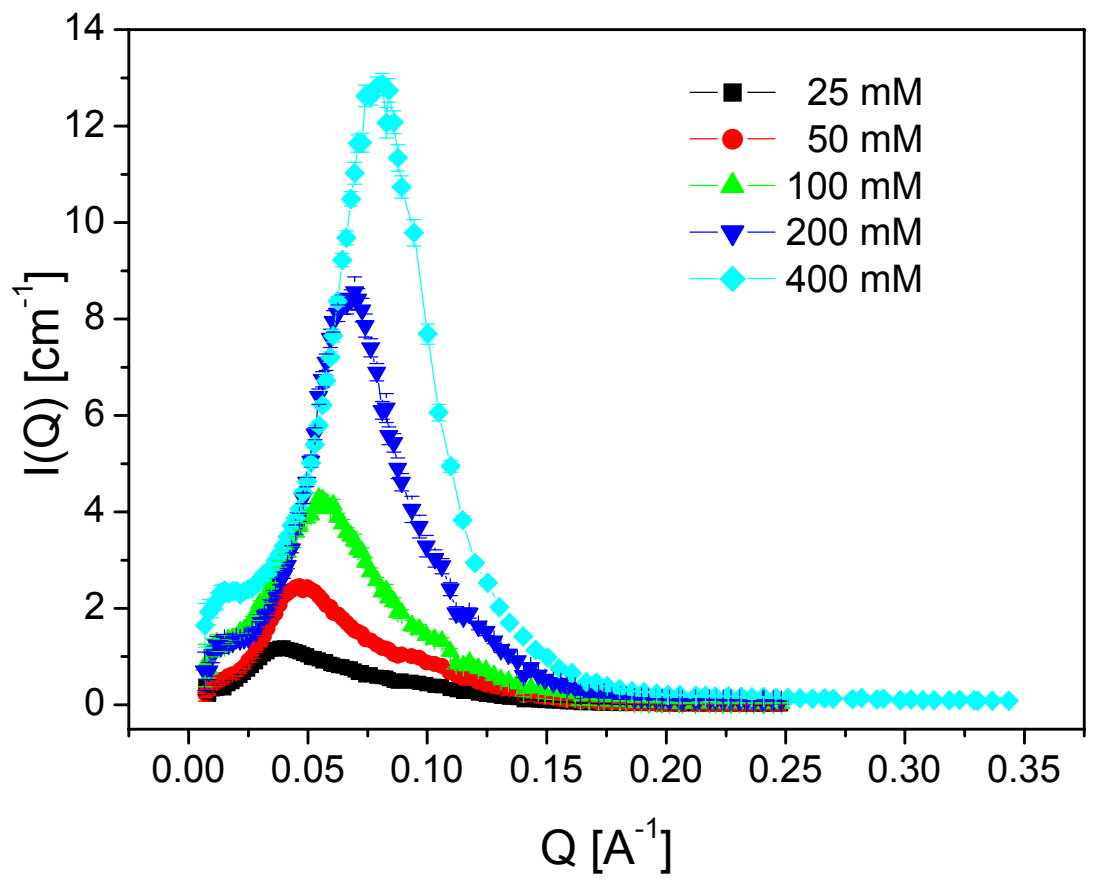

Figure 4. 4: Representative SANS intensity profiles at different TTAB concentration 
The intensity profiles are dominated by a well defined correlation peak that is due to a pronounced interaction between the charged micelles. Hayter and Penfold model (already discussed in chapter 3 ) is used to fit the SANS data and the fitted curves for form factor and the structure factor are also shown in Figure 4.3. In the entire concentration regime the fits were slightly improved if ellipsoidal micelles are taken into account rather than spherical ones. So, the aggregates are described as prolate ellipsoids with half axes $a=b=20.2 \AA$ and $c=28.9 \AA$, respectively. The aggregation number is determined from the volume of the ellipsoid $V=4 \pi / 3 a^{2} c$ and the effective volume of a single surfactant molecule $V_{0}$ $=550 \AA^{3}$ [Zana_1983, Zielinski_1987] to be about 90 at ambient conditions in close agreement with the findings of Gorski and Kalus [Gorski_2001]. The fit results are collected in Table 4.1. While the small axis of these prolate ellipsoids as well as the total charge ' $Z_{0}$ ' are found to be almost independent of surfactant concentrations, the large axis increases from $28.9 \AA$ at $25 \mathrm{mM}$ to $57.2 \AA$ at 673 $\mathrm{mM}$ as shown in the Figure 4.5. Consequently the aggregation number ' $\mathrm{N}_{\mathrm{agg}}$ ' increases from 90 to 203 which is also shown in the same figure (scale on right side).

\begin{tabular}{|c|c|c|c|c|c|c|}
\hline $\mathbf{C}_{\text {TTAB }} / \mathbf{m M}$ & $\mathbf{a}, \mathbf{b} / \AA$ & $\mathbf{c} / \AA$ & $\mathbf{Z}_{\mathbf{o}}$ & $\mathbf{N}_{\mathbf{a g g}}$ & $\alpha$ & $\chi^{2}$ \\
\hline 25 & 20.2 & 28.9 & 23 & 90 & 0.25 & 1.8 \\
\hline 50 & 19.7 & 31.5 & 22 & 93 & 0.24 & 1.7 \\
\hline 74 & 19.9 & 33.8 & 22 & 102 & 0.22 & 1.5 \\
\hline 149 & 20.0 & 34.4 & 22 & 105 & 0.21 & 3 \\
\hline 300 & 20.3 & 38.0 & 22 & 119 & 0.18 & 7 \\
\hline 375 & 20.6 & 42.6 & 21 & 138 & 0.15 & 8 \\
\hline 524 & 20.6 & 49.6 & 21 & 160 & 0.13 & 11 \\
\hline 595 & 21.1 & 50.8 & 20 & 172 & 0.12 & 14 \\
\hline 673 & 21.6 & 57.2 & 22 & 203 & 0.11 & 12 \\
\hline
\end{tabular}

Table 4. 1: Table 4.1: Geometrical parameters of TTAB micelles at ambient conditions for different concentrations: $a, b$ and $c$ are the half axes of the ellipsoid, $Z_{o}$ and $N_{\text {agg }}$ are the average charge and the aggregation number, respectively. $\alpha$ is the degree of dissociation and $\chi^{2}$ the mean squared deviation of the fit 


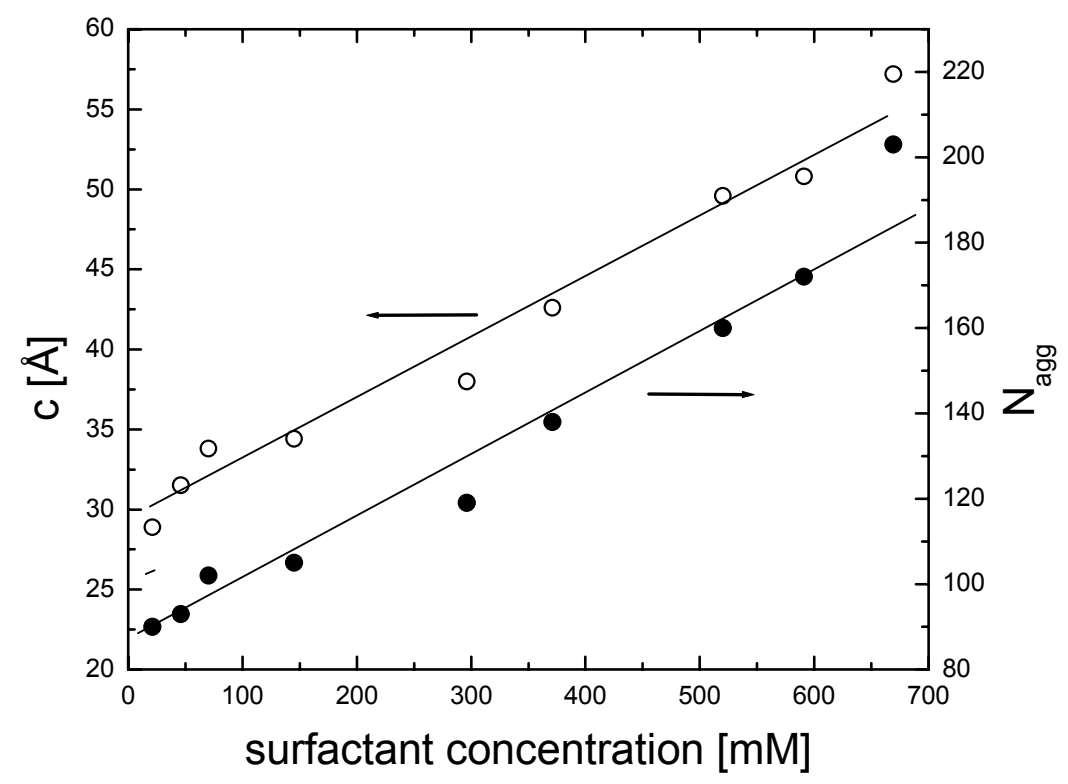

Figure 4. 5: Concentration dependence of the long micelle-axis $\mathrm{c}$ and the aggregation number $\mathrm{N}_{\text {agg }}$ at $25^{\circ} \mathrm{C}$

The position of the correlation peak ' $Q_{\max }$ ' can also be used to roughly estimate the density ' $n_{\text {mic }}$ ' of micelles according to Chen et al. [Chen_1988].

$$
n_{\text {mic }}=f Q_{\text {max }}^{3} \text {. }
$$

The factor $f$ depends on the local structure of the micellar solution and is to be $2.88 \times 10^{-3}$ for a large variety of micellar systems. From Figure $4.3, Q_{\max }$ is determined as $0.038 \AA^{-1}$ and correspondingly, the obtained density of micelles is $1.58 \times 10^{17} \mathrm{~cm}^{-3}$. Using the surfactant concentration $\mathrm{c}$, the aggregation number can be calculated as

$$
\mathrm{N}_{\text {agg }}=(\mathrm{c}-\mathrm{cmc}) \mathrm{N}_{\mathrm{L}} / \mathrm{n}_{\text {mic }}
$$

( $\mathrm{N}_{\mathrm{L}}$ is Avogadro number) which yields a value of 80 , somewhat smaller than the value determined from the geometrical considerations. This simple phenomenological approach is however, no longer applicable at higher concentrations and is demonstrated as follows.

Figure 4.4, presents the shift in the position of the correlation peak ' $Q_{\max }$ ' to the higher wave vector (higher $Q$ value) with increase of surfactant concentration, 
this type of concentration dependence is another indication of the growth of micelle. If there were no change in the aggregation number, a variation of the peak position $Q_{\max }$ proportional to $c^{1 / 3}$ would be expected due to the increase of the number density of micelles. As shown in Figure 4.6, however, $Q_{\max }$ exhibits a considerably weaker concentration dependence that can be described by a power law with exponent $0.244 \pm 0.004$ since the micelles are becoming larger in volume. A quantitative determination of the aggregation number merely from $Q_{\max }$ according to equations (1) and (2) is not easily possible since the maximum of the scattered intensity (being the product of the form factor and the structure factor) does not coincide with the maximum of the structure factor. Rather, it is shifted to smaller wave vectors by several percent depending on the mean intermicellar distance. This effect becomes more and more important for larger concentrations when the maximum of the structure factor appears at rather large $Q$, in a region where the form factor is strongly decreasing. Hence, the concentration dependence of $Q_{\max }$ as illustrated in the Figure 6 is the result of several competing effects and it seems surprising that such a clear power law behaviour is obtained.

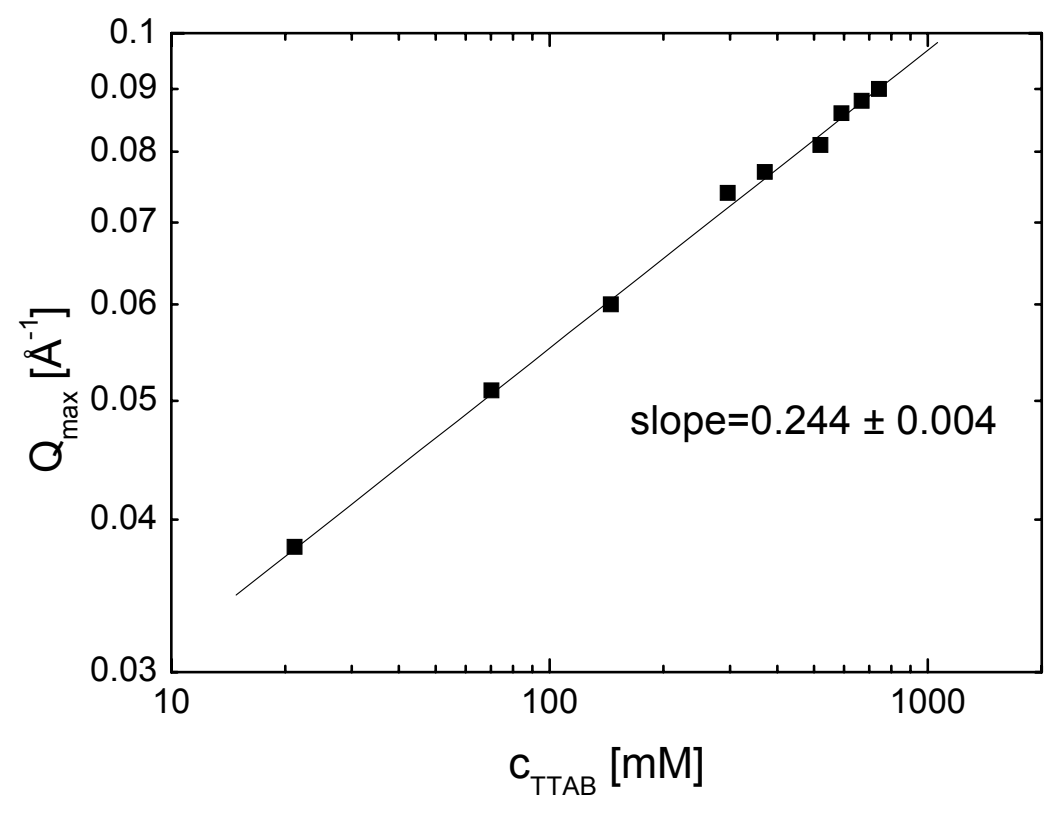

Figure 4. 6: Concentration dependence of the correlation peak position $Q_{\max }$ 


\subsection{Influence of temperature}

The configurations of surfactant molecules present in the micelles show considerable changes with temperature. Experiments are performed in wide range of temperature $\left(2{ }^{\circ} \mathrm{C}\right.$ to $\left.70{ }^{\circ} \mathrm{C}\right)$. For making it easy to understand the influence of temperature, first, the Raman spectra obtained above the room temperatures $\left(20^{\circ} \mathrm{C}\right.$ to $\left.70{ }^{\circ} \mathrm{C}\right)$ are presented. Spectral changes are well reflected in the band shape, shift in frequencies, full width half maxima (fwhm) and peak height ratios of the selected vibrational bands. Raman spectra of $\mathrm{CH}_{2} / \mathrm{CH}_{3}$ bending regime obtained at different temperatures are shown in Figure 4.7a. First, spectra are recorded with increasing temperature (the spectrum at $25{ }^{\circ} \mathrm{C}$ see the open circle spectra, and spectrum at $70{ }^{\circ} \mathrm{C}$ ) and then decreasing temperature. The intensity of a peak around $1440 \mathrm{~cm}^{-1}$ (see the arrow) assigned for the $\mathrm{CH}_{2}$ bending mode decreases with increase of temperature. The same peak gains intensity with decrease of temperature. The spectra at $25{ }^{\circ} \mathrm{C}$, both from before heating to $70^{\circ} \mathrm{C}$ i.e. spectra with open circles and after cooling from $70{ }^{\circ} \mathrm{C}$ resembles almost the same in intensity, hence, it is reversible.

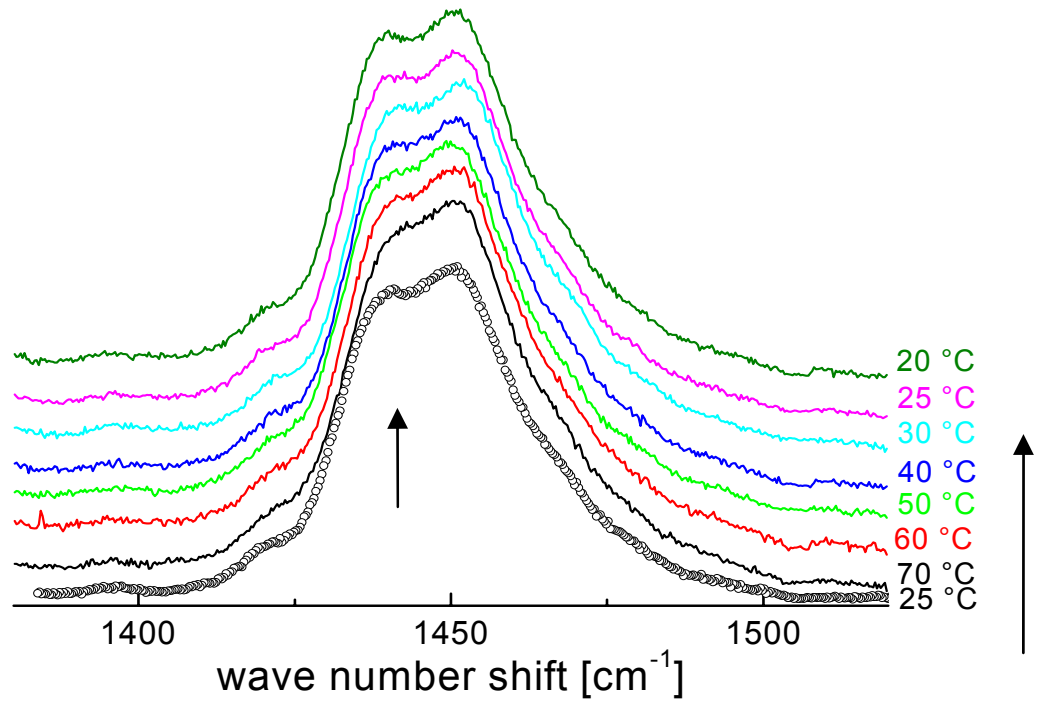

Figure 4. 7a: Changes in the shape of the Raman spectra of $0.4 \mathrm{M}$ TTAB with temperature (spectra are shifted vertically for clarity) 
The spectral regime corresponding to $\mathrm{C}-\mathrm{C}$ stretching vibrations is shown in Figure $4.7 \mathrm{~b}$ and $4.7 \mathrm{c}$. Figure $4.7 \mathrm{~b}$ cover the spectral regime from $1000 \mathrm{~cm}^{-1}$ to $1110 \mathrm{~cm}^{-1}$ with a peak around $1064 \mathrm{~cm}^{-1}$ corresponds to trans and the broad peak around $1085 \mathrm{~cm}^{-1}$ corresponds to the gauche conformations. The intensity of the $1064 \mathrm{~cm}^{-1}$ peak decreases and that of $1085 \mathrm{~cm}^{-1}$ increases with the increase in temperature (see spectra at $25{ }^{\circ} \mathrm{C}$ open circle and $70{ }^{\circ} \mathrm{C}$ ). This behavior is reversible with decrease of temperature, trans band gains intensity over the gauche band. It is clearly seen from the spectrum at $30^{\circ} \mathrm{C}$ that the intensity of both the bands is same (shown with horizontal dotted line). The spectra at $25{ }^{\circ} \mathrm{C}$, both from before heating to $70^{\circ} \mathrm{C}$ i.e. spectra with open circles and after cooling from $70{ }^{\circ} \mathrm{C}$ resemble almost the same. More flexible chains are seen at higher temperature (see below).

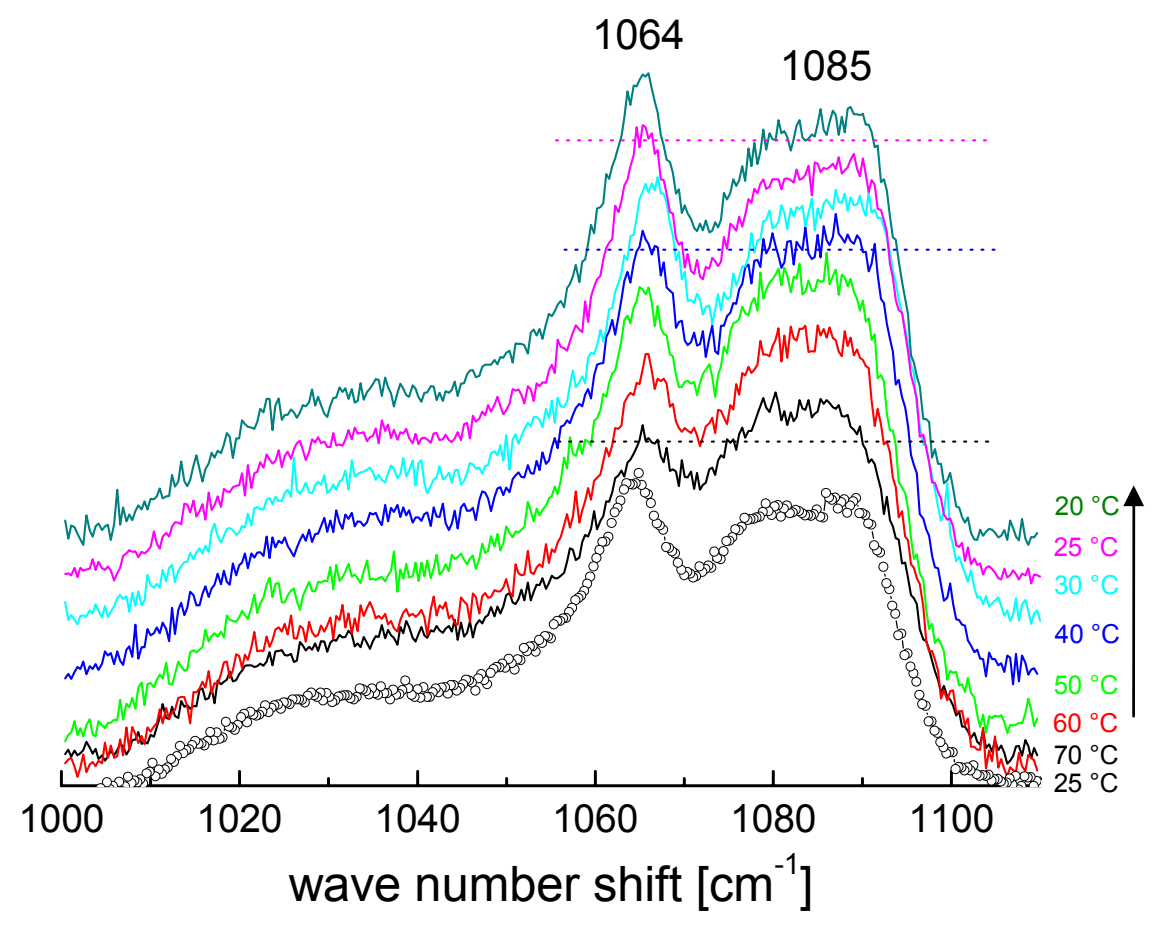

Figure 4. 7b: Effect of temperature on Raman spectrum of 0.4 M TTAB. Horizontal dotted lines to show the change in peak heights with temperature, arrow indicates spectrum recorded with decrease of temperature (spectra are shifted vertically for clarity) 
Figure $4.7 \mathrm{c}$ covers the spectral regime from $1110 \mathrm{~cm}^{-1}$ to $1150 \mathrm{~cm}^{-1}$ with a peak around $1123 \mathrm{~cm}^{-1}$ corresponding to trans and peak around $1140 \mathrm{~cm}^{-1}$ corresponds to the gauche conformations. The intensity of the $1123 \mathrm{~cm}^{-1}$ peak is almost equal to the peak around $1140 \mathrm{~cm}^{-1}$. Figure $4.7 \mathrm{c}$ confirms the flexibility of alkyl chain at higher temperature. This behavior is reversible with decrease of temperature, trans band gains intensity over the gauche band. The spectra at 25 ${ }^{\circ} \mathrm{C}$, both from before heating to $70{ }^{\circ} \mathrm{C}$ i.e. spectra with open circles and after cooling from $70^{\circ} \mathrm{C}$ resemble almost the same. Ratio of peak heights of trans $1123 \mathrm{~cm}^{-1}$ to gauche $1140 \mathrm{~cm}^{-1}$ is plotted against temperature in Figure $4.7 \mathrm{~d}$. This plot illustrates that the fraction of trans conformations increases with decrease of temperature. Ratio before heating to $70{ }^{\circ} \mathrm{C}$ is also shown (red triangle).
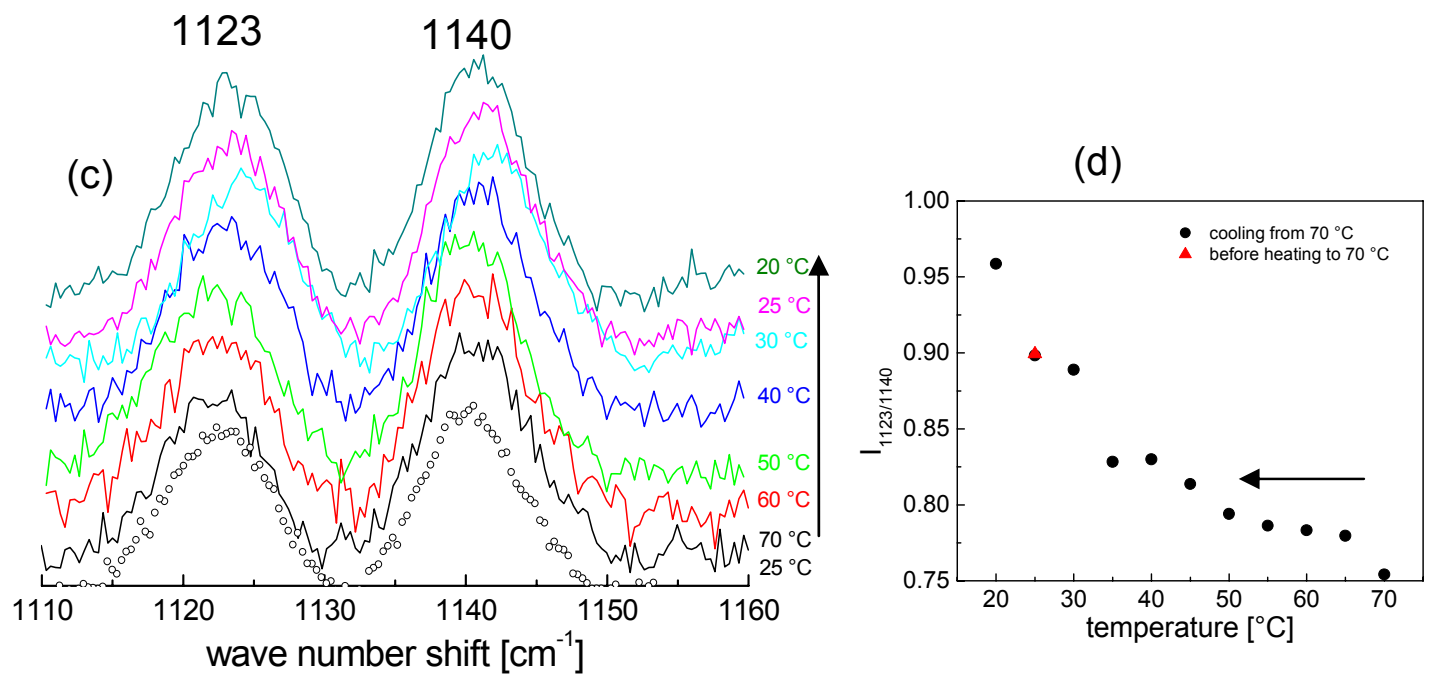

Figure 4. 7c and 4.7d: (c) change in trans and gauche conformations with temperature. Arrow indicates measurement done with decreasing temperature (spectra are shifted vertically for clarity), (d) ratio of $1123 \mathrm{~cm}^{-1}$ (trans) to $1140 \mathrm{~cm}^{-1}$ (gauche) peak, arrow indicates measurement done with decreasing temperature 
From above spectral results it is concluded that fraction of gauche conformation increases with increase in temperature and decreases with decrease in temperature in the temperature window of $20^{\circ} \mathrm{C}$ to $70{ }^{\circ} \mathrm{C}$.

Quantitative information in terms of aggregation number at different temperature can be obtained with the help of SANS intensity profiles. In section 4.2 it was mentioned that the position of the correlation peak ' $Q_{\max }$ ', in the SANS intensity profile can also be used (at low concentrations) to determine the aggregation number of micelles. Figure $4.8 \mathrm{a}$ is shown for the SANS intensity profile at 3 different temperatures. The $Q_{\max }$ shifts to higher wave vector ' $Q$ ' and the intensity of the SANS profiles decreases with increase in temperature due to the result of decrease in micellar size. Hence, the aggregation number as obtained from equation (1) and (2) decreases with increase in temperature as shown in Figure $8 \mathrm{~b}$ for $25 \mathrm{mM}$ and $50 \mathrm{mM}$ surfactant concentration (the difference in aggregation number for $25 \mathrm{mM}$, in Figure 4.8b and in Table 4.1 is because of two different methods used to calculate, which has already discussed in section 4.2). The decrease in aggregation number with increase in temperature is in good agreement with the findings of Gorski et al. [Gorski_2001].
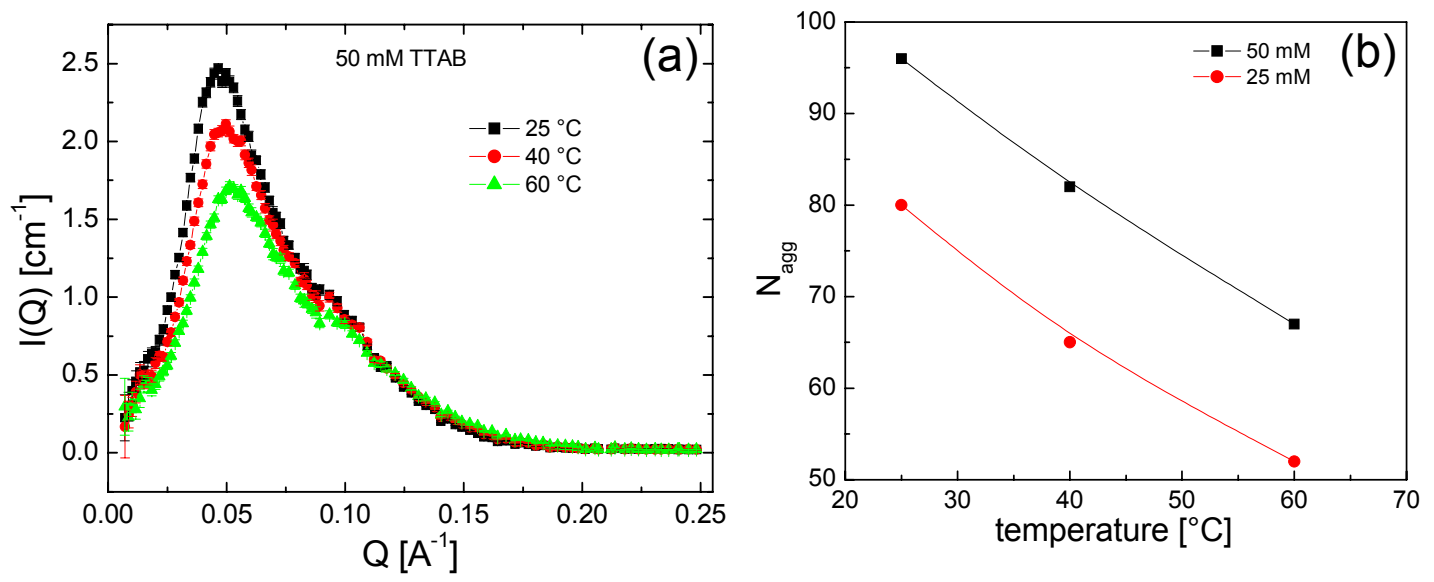

Figure 4. 8: (a) SANS intensity profiles for $50 \mathrm{mM}$ TTAB in $\mathrm{D}_{2} \mathrm{O}$ at 3 different temperatures, and (b) aggregation number of $25 \mathrm{mM}$ and $50 \mathrm{mM}$ TTAB at different temperatures 
The behaviors of micellar solutions in the temperature window of $20{ }^{\circ} \mathrm{C}$ to $2{ }^{\circ} \mathrm{C}$ are as follows. The phase transition from micellar solution to solid like /coagel/ crystalline phase is observed on cooling. The spectrum of this phase (below 5 ${ }^{\circ} \mathrm{C}$ ) resembles that of powder TTAB. Here onwards this phase is referred as solid phase. Figures $4.9 \mathrm{a}, 4.9 \mathrm{~b}$ and $4.9 \mathrm{c}$ show the Raman spectra of TTAB in three different spectral regimes of longitudinal accordion modes (LAM), C-H deformation and $\mathrm{C}-\mathrm{C}$ stretching, and $\mathrm{C}-\mathrm{H}$ stretching respectively. Spectra from micellar solution, after phase transition and powder sample are compared. Spectra for micelles and powder samples are measured at room temperatures. The tentative assignments of the spectrum are tabulated in Table 4.2. Cooling and heating cycles were performed for $0.4 \mathrm{M}$ aqueous TTAB solutions. Higher surfactant concentration was chosen because, in the temperature scale of $2{ }^{\circ} \mathrm{C}$ to $25{ }^{\circ} \mathrm{C}$, the phase transition from micelles to solid phase can be achieved without disturbing the system, in other words without inducing the precipitation (nucleation), whereas low surfactant concentrations $(<100)$ requires nucleation either by shaking or stirring. Figure $4.9 \mathrm{a}$ corresponds to the LAM, Figure $4.9 \mathrm{~b}$ to $\mathrm{C}-\mathrm{C}$ stretching, $\mathrm{CH}_{2} / \mathrm{CH}_{3}$ bending, and twisting modes, and that of Figure $4.9 \mathrm{c}$ corresponds to the $\mathrm{C}-\mathrm{H}$ stretching in methylene $\left(\mathrm{CH}_{2}\right)$ and methyl $\left(\mathrm{CH}_{3}\right)$ group.

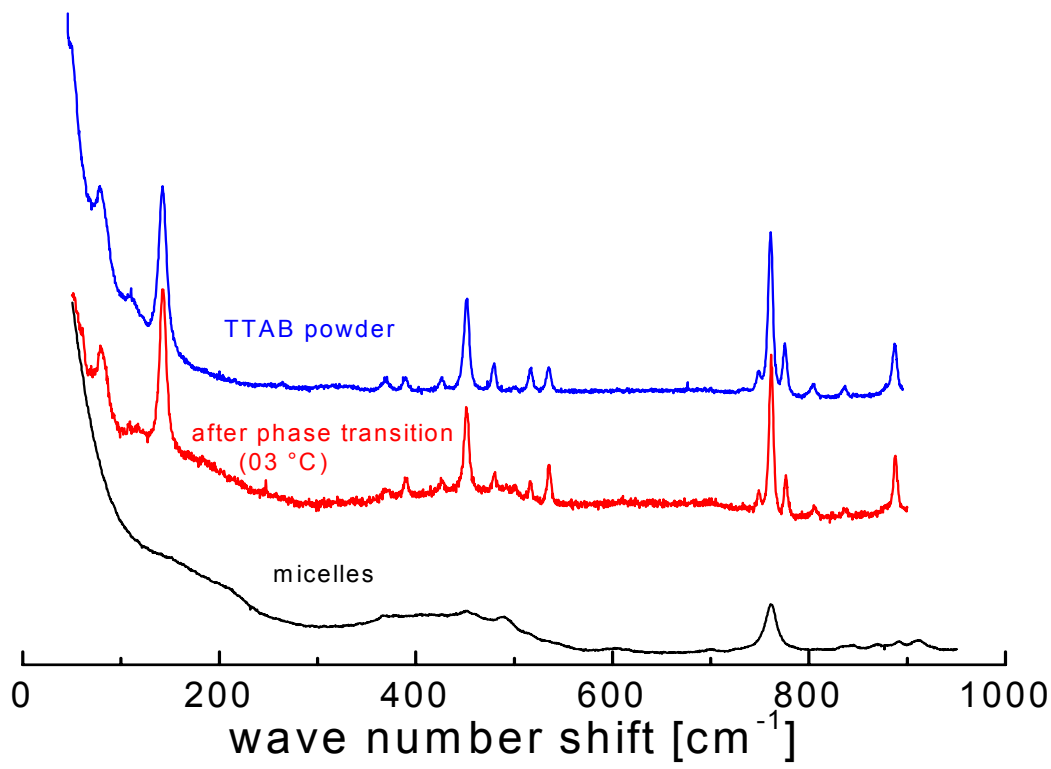

Figure 4. 9a: Longitudinal accordian modes at $20^{\circ} \mathrm{C}$ and $02{ }^{\circ} \mathrm{C}$ and comparison with the powder TTAB measured at room temperature 
Peak frequency, full width half maxima, and peak height ratios of these modes are very much sensitive at phase transition of the system. Temperature dependences of selected bands of these quantities are presented here in Figures $4.10-4.13$. The sharp changes were observed near $5{ }^{\circ} \mathrm{C}$ on cooling from $25^{\circ} \mathrm{C}$.

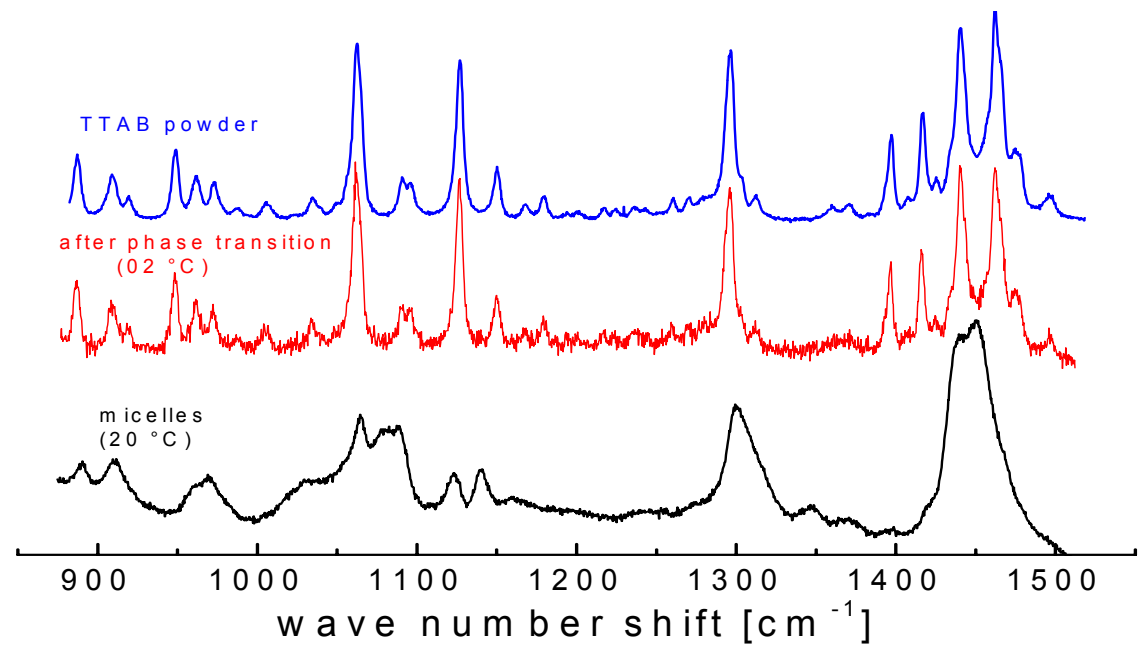

Figure 4.9b: $\mathrm{C}-\mathrm{H}$ deformation and $\mathrm{C}-\mathrm{C}$ stretching regime at $20{ }^{\circ} \mathrm{C}$ and $2{ }^{\circ} \mathrm{C}$ and comparison with powder TTAB sample measured at room temperature

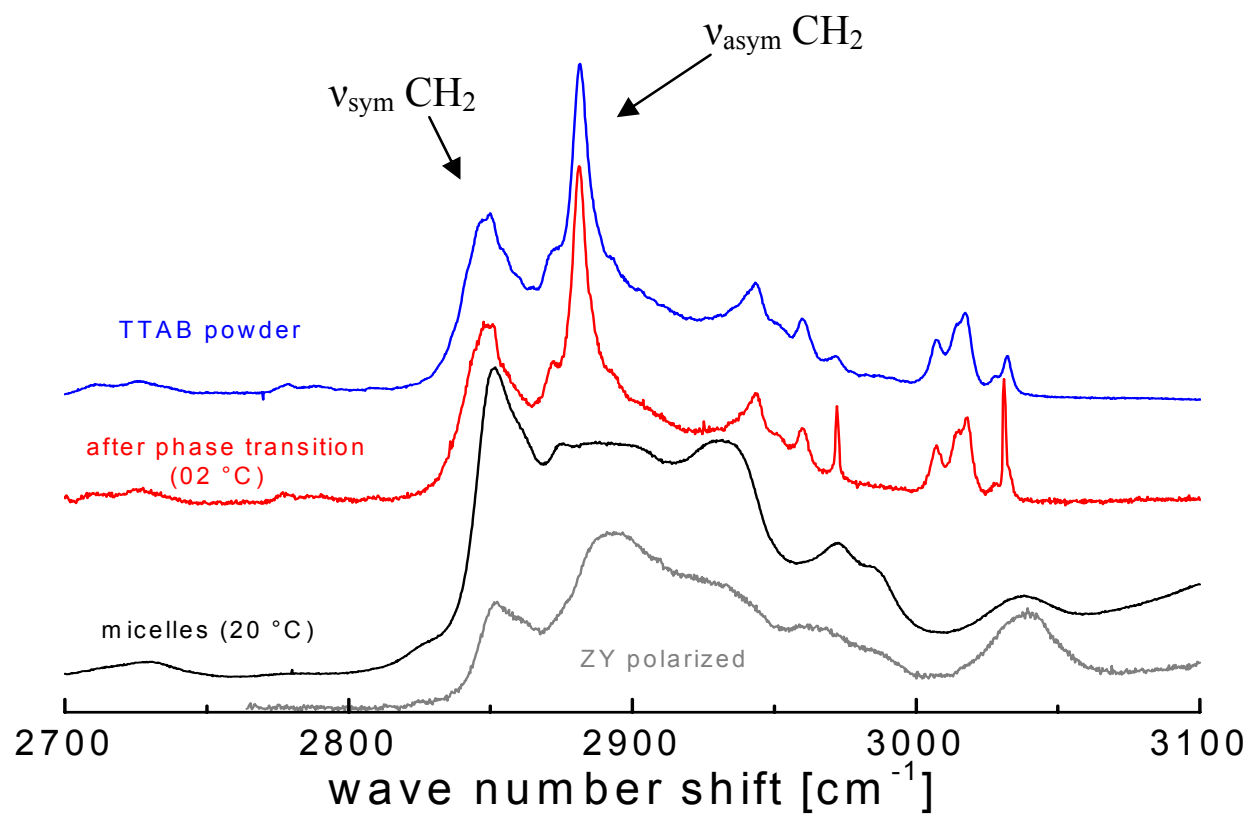

Figure 4.9c: $\mathrm{C}-\mathrm{H}$ stretching regime at $20^{\circ} \mathrm{C}$ and $02{ }^{\circ} \mathrm{C}$ and comparison with powder TTAB measured at room temperature, ZY polarized micellar spectra also shown for asymmetric stretching 
The dissolution of solid phase appears near $12{ }^{\circ} \mathrm{C}$ on heating, the temperature at which the dissolution appears is known as critical micellization temperature (cmt). The discontinuities in peak frequency, fwhm, and height ratio are observed near $12{ }^{\circ} \mathrm{C}$ on heating indicating pronounced hysteresis.

\begin{tabular}{|c|c|c|c|c|}
\hline micelles & $\begin{array}{l}\text { after phase } \\
\text { transition }\end{array}$ & powder TTAB & tentative assignment & \\
\hline \multirow[t]{2}{*}{160} & 142 & 142 & & \\
\hline & $\begin{array}{l}451.6 \\
480 \\
516.5 \\
535 \\
749\end{array}$ & $\begin{array}{l}451.5 \\
479 \\
516.5 \\
535 \\
748\end{array}$ & Longitudinal accordion modes & \\
\hline 761 & $\begin{array}{l}761.6 \\
776.5\end{array}$ & $\begin{array}{l}761 \\
775.5\end{array}$ & $\mathrm{CH}_{3}$ rock from $\mathrm{N}^{+}\left(\mathrm{CH}_{3}\right)_{3}$ & \\
\hline \multicolumn{5}{|l|}{835} \\
\hline 869 & 887 & 887 & $\mathrm{CH}_{3}$ rock (terminal methyl) & \\
\hline 891 & 909 & 909 & $\mathrm{C}-\mathrm{N}^{+}$stretch & \\
\hline 910 & $\begin{array}{l}948.4 \\
961.8\end{array}$ & $\begin{array}{l}948.5 \\
961.5\end{array}$ & & \\
\hline 957 & 972.4 & 973 & $\mathrm{CH}_{2}$ rock & \\
\hline 968 & 987.4 & 987.5 & & \\
\hline 1064 & 1062 & 1062 & $\mathrm{C}-\mathrm{C}$ stretching $+\mathrm{CH}_{2}$ wag & \\
\hline 1079 & 1090 & 1090 & C-C stretching & \\
\hline 1089 & 1096 & 1096 & & \\
\hline 1123.4 & 1127 & 1127 & $\mathrm{C}-\mathrm{C}$ stretching $+\mathrm{CH}_{2}$ wag from & \\
\hline 1140.5 & 1150 & 1150 & $\left(\mathrm{TG}_{\mathrm{m}} \mathrm{T}\right)$ with $\mathrm{m}$ large $\mathrm{CH}_{2}$ rock & \\
\hline \multirow[t]{2}{*}{1300} & 1296 & 1296 & $\mathrm{CH}_{2}$ twist & \\
\hline & 1397 & 1397 & $\mathrm{CH}_{3}$ bend & \\
\hline 1421 & 1416.2 & 1416.7 & & \\
\hline 1437.5 & 1440.6 & 1440.7 & & \\
\hline 1450.5 & 1462.4 & 1462 & & \\
\hline \multirow[t]{3}{*}{1462} & 1466.5 & 1466.5 & $\mathrm{CH}_{2}$ bend & \\
\hline & 1475.8 & 1475.8 & & \\
\hline & 1496.5 & 1496 & & \\
\hline 2850.5 & 2848.5 & 2848.5 & $\mathrm{C}-\mathrm{H}$ sym stretch of $\mathrm{CH}_{2}$ & $83 \mathrm{~b}$ \\
\hline $\begin{array}{l}2874 \\
2889(a)\end{array}$ & 2881 & 2881 & $\begin{array}{l}\mathrm{C}-\mathrm{H} \text { sym stretch of } \mathrm{CH}_{3} \\
\mathrm{C}-\mathrm{H} \text { asym stretch of } \mathrm{CH}_{2}\end{array}$ & ng_1983b \\
\hline \multirow[t]{2}{*}{2829.5} & 2943 & 2943 & -त asym stretch or $\mathrm{CH}_{2}$ & \\
\hline & 2960 & 2960 & & \\
\hline \multirow{2}{*}{$\begin{array}{l}2972 \\
2988\end{array}$} & 2972 & 2972 & $\mathrm{C}-\mathrm{H}$ asym stretch of $\mathrm{CH}_{3}$ & Mantsch_1982 \\
\hline & 3007 & 3007 & & \\
\hline \multirow[t]{2}{*}{3036} & 3017.5 & 3017 & $\mathrm{C}-\mathrm{H}$ sym stretch of $\mathrm{CH}_{3}$ & Hattori_1999, \\
\hline & 3032 & 3032 & from head group & Mantsch_1982 \\
\hline
\end{tabular}

Table 4. 2: Tentative assignment for vibration bands of tetradecyltrimethylammonium Bromide surfactant in micelles, after phase transition and powder form, assignments are on the basis of literature. All the assignments taken from Kalyanasundaram_1976, unless otherwise referenced against the frequencies. (a) is measured in zy polarization 
Each spectral regime and the information are discussed separately.

\section{C-H deformation regime:}

Figure $4.9 \mathrm{~b}$ corresponds to $0.4 \mathrm{M}$ aqueous TTAB solution in the spectral regime of $970 \mathrm{~cm}^{-1}$ to $1550 \mathrm{~cm}^{-1}$, the main features of this regime are $\mathrm{CH}_{2}$ scissoring, twisting, wagging and the very important, skeletal C-C stretching. The strong and broad $\mathrm{CH}_{2} / \mathrm{CH}_{3}$ bending peak is observed near $1450 \mathrm{~cm}^{-1}$ with shoulders on right and left of this peak. This broad band splits up into several sharp peaks near the phase transition temperature and such spectral features resemble the spectrum of powder TTAB which was measured at room temperature.

The peak around $1300 \mathrm{~cm}^{-1}$ in micellar phase is quite strong and assigned for $\mathrm{CH}_{2}$ twisting mode. The position and width of this band is sensitive to the change in temperature. The change in peak position and width are used to monitor the conformation and the chain mobility. This peak shows decrease in frequency with decrease in temperature, and near $5{ }^{\circ} \mathrm{C}$ it shows a sharp drop as shown in Figure 4.10. The full width half maxima of this band decreases on cooling and a sharp decrease near the phase transition temperature $5^{\circ} \mathrm{C}$ (Figure $4.11 \mathrm{~b}$ ).

(a)

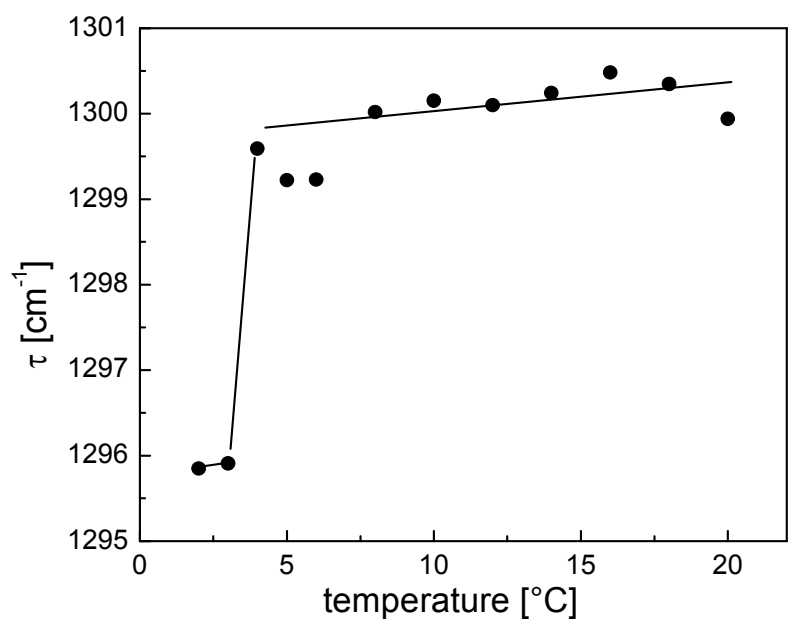

Figure 4. 10: Frequency shifts of $0.4 \mathrm{M}$ TTAB as a function of temperature $\mathrm{CH}_{2}$ twisting mode 
In the same Figure $4.11 \mathrm{~b}$, a full width half maximum of experiments with heating is also shown (see the red circles and the red line as eye guide). This clearly shows the pronounced hysteris of about $8{ }^{\circ} \mathrm{C}$ with cooling and heating. For the case of $n$-alkanes solid-liquid phase transition the twisting band shows an increase in frequency of about $5 \mathrm{~cm}^{-1}$ [Brown_1987] which is close to present findings in TTAB. The frequency and width of this band in powder TTAB and after the phase transition of TTAB from micelles are almost identical.

C-C stretching regime is another important spectral part which covers the frequency range $1000 \mathrm{~cm}^{-1}$ to $1150 \mathrm{~cm}^{-1}$. This regime has been widely used in monitoring conformational changes in n-alkane [Brown_1987, Snyder_1982], fatty acids [Levin_1981, Wong_1982, Akutsu_1981, Wong_1983] and lipids [Lippert_1971, Yellin_1977, Gaber_1977]. The peaks around $1064 \mathrm{~cm}^{-1}$ and $1123 \mathrm{~cm}^{-1}$ are assigned to asymmetric and symmetric C-C stretching and these bands are associated with trans conformer of the alkyl chain of the TTAB. Broad band near $1085 \mathrm{~cm}^{-1}$ (Figure 4.9b) is assigned for $\mathrm{C}-\mathrm{C}$ stretching associated to gauche conformation, in micellar phase this band has a shoulder, and near the phase transition it appears as doublet and has very small intensity. Below the phase transition temperature the asymmetric $\mathrm{C}-\mathrm{C}$ band shows decrease in frequency of about $2 \mathrm{~cm}^{-1}$ and that of symmetric $\mathrm{C}-\mathrm{C}$ band shows an increase of about $4 \mathrm{~cm}^{-1}$.
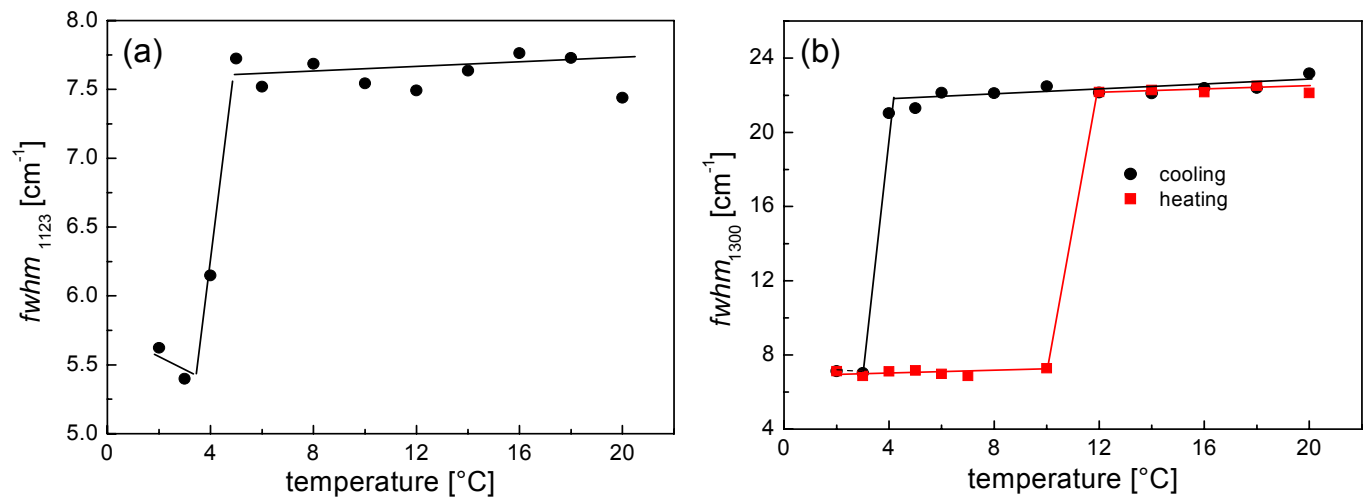

Figure 4. 11: Full width half maxima as a function of temperature (a) $\mathrm{C}-\mathrm{C}$ stretching $\left(1123 \mathrm{~cm}^{-1}\right)$ and $(\mathrm{b}) \mathrm{CH}_{2}$ twisting $\left(1300 \mathrm{~cm}^{-1}\right)$ 
These bands show considerable changes in full width half maximum near phase transition temperature. The fwhm of symmetric $1123 \mathrm{~cm}^{-1}$ band as a function of temperature is shown in Figure 11a. The separation of selected peaks can also be used to monitor the phase transition. The asymmetric $1064 \mathrm{~cm}^{-1}$ and symmetric $1123 \mathrm{~cm}^{-1} \mathrm{C}-\mathrm{C}$ stretching (trans), and gauche $1140 \mathrm{~cm}^{-1}$ and symmetric trans $1123 \mathrm{~cm}^{-1}$ peak frequency differences are shown in Figure $4.12 \mathrm{a}$ and $4.12 \mathrm{~b}$ respectively. The corresponding peak frequency differences in powder TTAB sample are compared with peak frequency difference after phase transition and are found to be the same.

The peak height ratio of trans and gauche conformers gives the structural changes of the micelles. Figure 4.13, shows the ratio of trans to gauche peak height. Ratio of intensity height of $1123 \mathrm{~cm}^{-1}$ to $1085 \mathrm{~cm}^{-1}, 1064 \mathrm{~cm}^{-1}$ to $1085 \mathrm{~cm}^{-1}$ and $1123 \mathrm{~cm}^{-1}$ to $1140 \mathrm{~cm}^{-1}$ are plotted and all these ratios show quite similar behaviors with temperature. Spectral region $1100 \mathrm{~cm}^{-1}$ to $1160 \mathrm{~cm}^{-1}$ was fitted with Gaussian function, whereas the spectral region $1000 \mathrm{~cm}^{-1}$ to $1110 \mathrm{~cm}^{-1}$ was fitted with Lorentzian functions (attempts to fit the both spectral regime with a same function either Lorentzian or Gaussian was not very successful). Gauche band $1085 \mathrm{~cm}^{-1}$ was fitted with two peaks and the intensity of both peak is considered $(1080+1090$, as shown in Figure 4.13a, green and blue symbols).
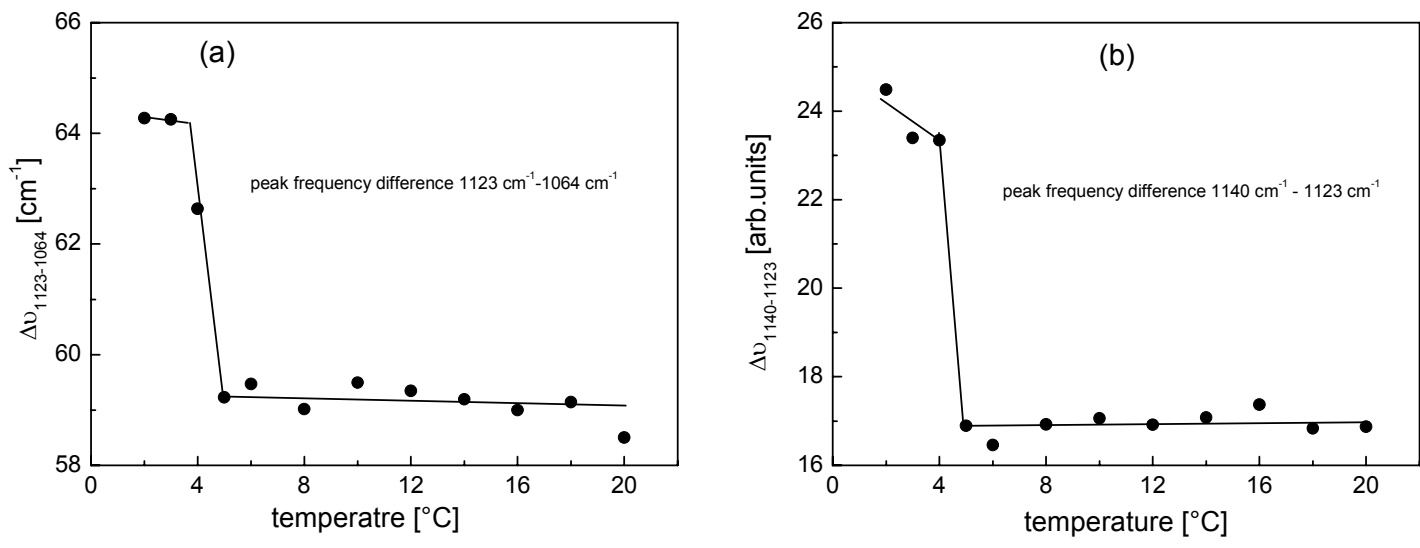

Figure 4. 12: Peak frequency differences as a function of temperature (a) symmetric $\left(1123 \mathrm{~cm}^{-1}\right)$ and asymmetric $\left(1064 \mathrm{~cm}^{-1}\right) \mathrm{C}-\mathrm{C}$ stretch (b) $1140 \mathrm{~cm}^{-1}$ and $1123 \mathrm{~cm}^{-1}$ 
(a)

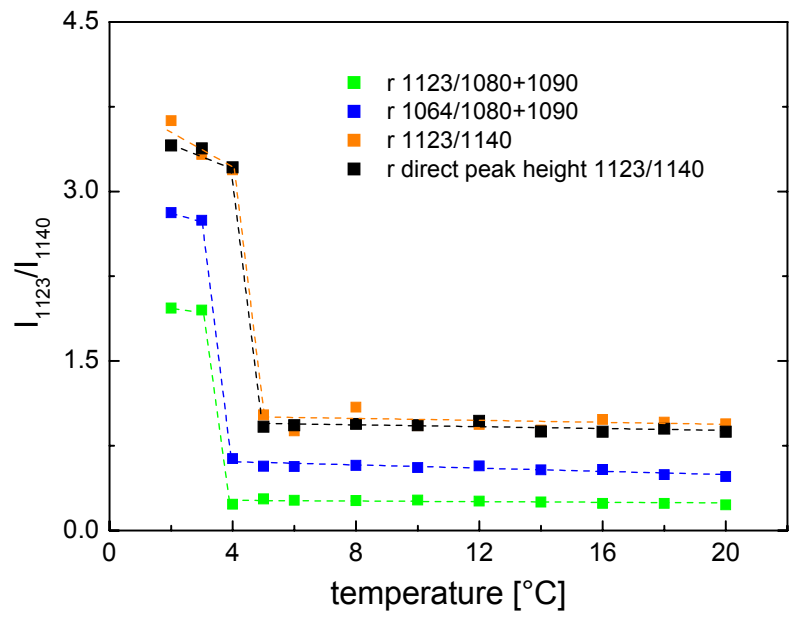

Figure 4. 13: Ratios peak height of trans to gauche in $\mathrm{C}-\mathrm{C}$ stretching region (symbols are described in text)

The direct peak height was also used to find the ratios of trans to gauche population. In Figure $13 \mathrm{a}$, the ratio of $1123 \mathrm{~cm}^{-1}$ to $1140 \mathrm{~cm}^{-1}$ with both the methods is shown. Below the transition temperature, the spectra indicate the presence of maximum number of trans conformations of the alkyl chain. Considerable decrease in gauche peak intensity and increase in trans peak intensity was observed below the transition temperature. This behavior is reflected in the plots of ratio of trans to gauche conformation and suggests that the population of trans conformation is maximum in solid phase.

\section{C-H stretching region}

This regime has been well investigated for the study of model and biological membranes [Gaber_1977, Snyder_1980, Wong_1983, Kobayashi_1980]. For 0.4 $\mathrm{M}$ TTAB aqueous micellar solution, the $\mathrm{C}-\mathrm{H}$ stretching regime has also been used to understand the dynamics of micellar solution as the symmetric and asymmetric $\mathrm{C}-\mathrm{H}$ stretches are much sensitive to temperature. As shown in the Figure $4.9 \mathrm{c}$, the band near $2850.5 \mathrm{~cm}^{-1}$ has been assigned for $\mathrm{C}-\mathrm{H}$ symmetric stretching and at 2889 and $2930 \mathrm{~cm}^{-1}$ two broad Fermi resonance bands [Snyder_1978, Snyder_1979, Snyder_1982] are observed. For micellar solution, these broad bands are analyzed by polarization measurement and it is found that 
asymmetric $\mathrm{C}-\mathrm{H}$ stretching band has overlapped with Fermi resonance band which is centered at $2890 \mathrm{~cm}^{-1}$. The band $2875 \mathrm{~cm}^{-1}$ observed as a shoulder to symmetric $\mathrm{C}-\mathrm{H}$ stretching band is assigned for methyl symmetric $\mathrm{C}-\mathrm{H}$ stretching band. Both the frequency and width of symmetric and asymmetric $\mathrm{C}-\mathrm{H}$ stretching bands show considerable changes near the phase transition temperature (near 5 ${ }^{\circ} \mathrm{C}$ ). Figure 4.14 is presented with the spectra measured in zy polarization mode in order to follow the methylene $\mathrm{C}-\mathrm{H}$ asymmetric $\left(2889 \mathrm{~cm}^{-1}\right)$ stretching band. Another significant change is also seen near $3036 \mathrm{~cm}^{-1}$ which correspond to $\mathrm{C}-\mathrm{H}$ stretching of $\mathrm{CH}_{3}$ groups present at the head group. Following the vertical line near $2889 \mathrm{~cm}^{-1}$ and $3036 \mathrm{~cm}^{-1}$ peaks, it clearly shows that the frequency decreases on cooling and a sharp decrease near phase transition temperature. A significant decrease in width of these bands is observed near phase transition temperature. Near the phase transition temperature on cooling asymmetric $\mathrm{C}-\mathrm{H}$ stretching band evolve as a sharp peak with larger intensity than the symmetric $\mathrm{C}-\mathrm{H}$ stretching band.

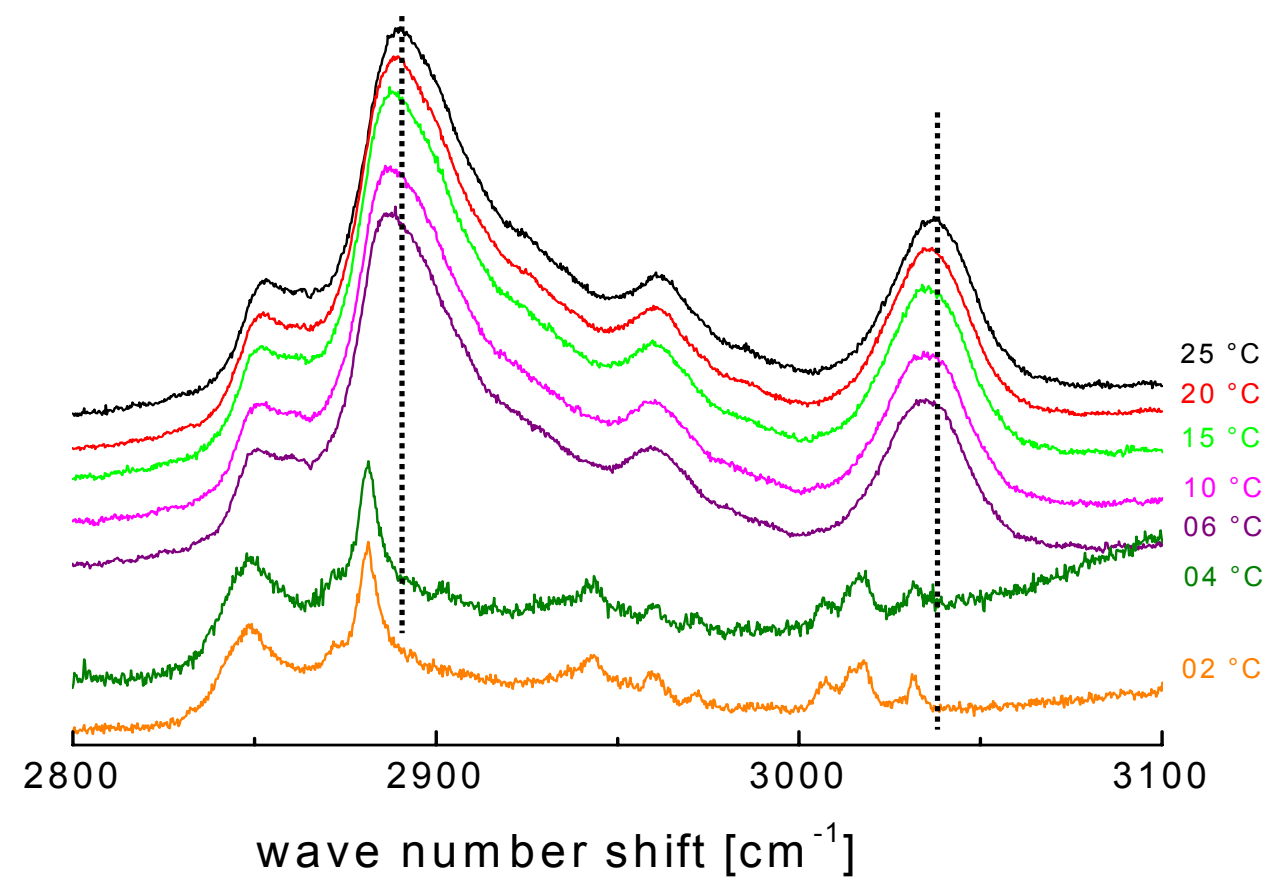

Figure 4. 14: Changes in the methylene asymmteric $\mathrm{C}-\mathrm{H}$ stretching band with temperature, measurements done in zy polarization mode for $0.4 \mathrm{M}$ TTAB 
Frequency of $\mathrm{C}-\mathrm{H}$ symmetric stretching and intensity height ratio of asymmetric and symmetric $\mathrm{C}-\mathrm{H}$ stretching bands against temperature are shown in Figure $4.15 \mathrm{a}$ and $4.15 \mathrm{~b}$ respectively. As shown in Figure 4.15a the frequency decreases on cooling and a sharp decrease near the phase transition temperature. Figure $15 \mathrm{~b}$ also presents the peak height ratio of heating measurements to show the hysteresis of about $8{ }^{\circ} \mathrm{C}$.

This decrease of $3036 \mathrm{~cm}^{-1}$ peak frequency is in agreement with the findings of Hattori [Hattori_1999] who has showed Raman spectrum in 2600 to $3100 \mathrm{~cm}^{-1}$ regime for the dimeric bis \{quatarneryammonium bromide\} surfactants (where one surfactant with the spacer of six $\mathrm{CH}_{2}$ group, and an alkyl chain lengths of eight carbon atom and another surfactant with the spacer of two $\mathrm{CH}_{2}$ group and an alkyl chain lengths of six carbon atoms) in micelles, transparent gel phase and solid phase. With the careful inspection of the Raman spectra in these phases, it's observed that the peak around $3036 \mathrm{~cm}^{-1}$ decreases more than $10 \mathrm{~cm}^{-1}$ from micelles to solid phase. These results are in contradiction to the findings of Kawai [Kawai_1985], who has found using infrared (IR) measurements, an increase of about $2 \mathrm{~cm}^{-1}$ in $3028 \mathrm{~cm}^{-1}$ peak, which is assigned for $\mathrm{C}-\mathrm{H}$ stretching of $\mathrm{CH}_{3}$ group present at head group of surfactant Octadecyltrimethylammonium Chloride (OTAC) - water system. However, decrease of about $9 \mathrm{~cm}^{-1}$ was found for the asymmetric $\mathrm{C}-\mathrm{H}$ stretching band $\left(2923 \mathrm{~cm}^{-1}\right)$ moving from micelles (liquid crystal) to solid phase.
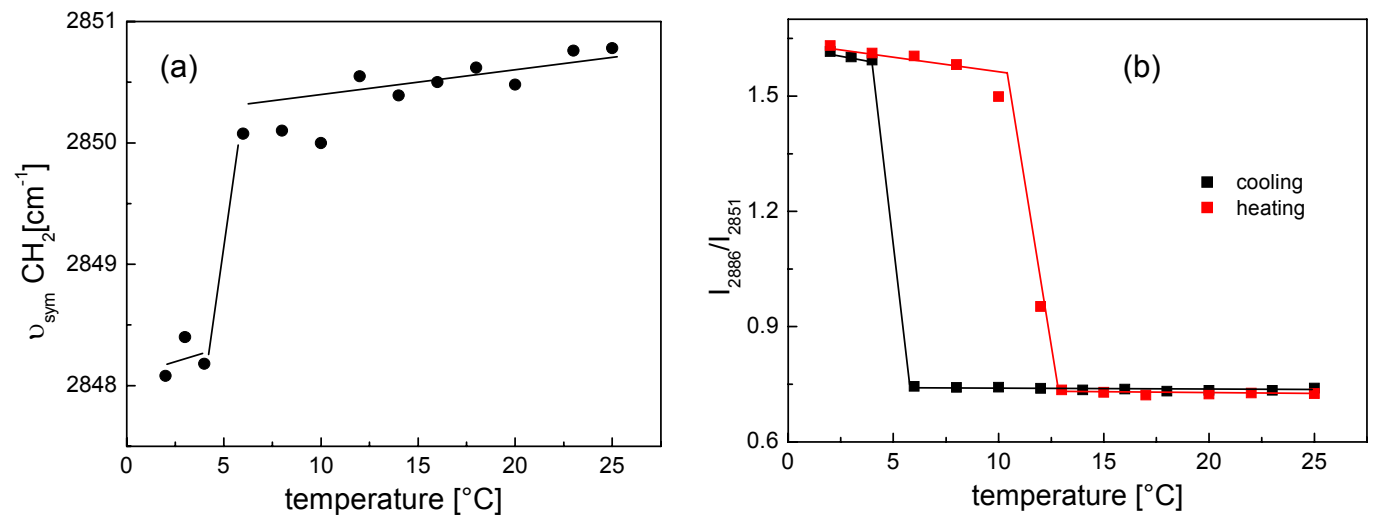

Figure 4. 15: Changes in $\mathrm{C}-\mathrm{H}$ stretching regime (a) shift in peak frequency, (b) ratio of peak height of asymmetric to symmetric bands (solid lines are to guide the eye) 


\section{Below $1000 \mathrm{~cm}^{-1}$ regime}

As shown in Figure 4.9a, the spectral region exhibits bands near $450 \mathrm{~cm}^{-1}$ (expanded portion is shown in Figure $4.2 \mathrm{c}$ ), $760 \mathrm{~cm}^{-1}, 900 \mathrm{~cm}^{-1}$ and $960 \mathrm{~cm}^{-1}$ (shown in Figure 4.9b) are assigned to $\mathrm{C}-\mathrm{N}$ stretching, $\mathrm{CH}_{3}$ rocking from head group region $\left(\mathrm{C}-\mathrm{N}^{+}\left(\mathrm{CH}_{3}\right)_{3}\right), \mathrm{CH}_{3}$ rocking from terminal methyl group, and $\mathrm{CH}_{2}$ rocking modes respectively. Near the phase transition temperature all bands become sharp and intense. In micellar phase, a broad band is observed near 150 $\mathrm{cm}^{-1}$, but near the phase transition temperature a sharp band is observed at about $142 \mathrm{~cm}^{-1}$. This band is assigned to longitudinal accordion mode (LAM).

\section{Discussion}

Aqueous micellar solution of $0.4 \mathrm{M}$ TTAB undergoes phase transition into solid/coagel/crystalline phase near $5{ }^{\circ} \mathrm{C}$ on cooling. The spectrum after the phase transition on cooling resembles that of spectrum of powder TTAB which is recorded at room temperature. The back transformation into liquid micelles as solution (critical micelle temperature) is observed near $12{ }^{\circ} \mathrm{C}$ on heating, showing pronounced hystereses of $8{ }^{\circ} \mathrm{C}$. This $\mathrm{cmt}$ is in good agreement with the findings of Kaneshina et al. [Kaneshina_1989] who determined using differential scanning calorimetric study for $30 \mathrm{mM}$ TTAB. The phase transition temperature on cooling or heating is well reflected by the discontinuities in peak frequency, full width half maxima, peak height ratio and difference in selected peak frequencies. Bartoli and Litovitz [Bartoli_1972] have reported the analysis of orientational broadening of Raman peaks shape in many liquids and it is noted that broadening of the anisotropic (depolarized) component Raman bands in condensed phase is largely due to the reorientational fluctuations in the molecules, while the width of isotropic component is not affected by the reorientational fluctuations [Bartoli_1972] but is strongly affected by interchain interaction [Abbott_1979, Kobayashi_1980]. Wong and Mantsch [Wong_1983a] have used similar interpretations to the temperature induced phase transition of sodium oleate. The 
conformation of alkyl chain is mainly reflected in skeletal C-C stretching (Figure $9 \mathrm{~b}$ ) and $\mathrm{C}-\mathrm{H}$ stretching (Figure 4.9c) bands. As the ratio of trans to gauche conformations (Figure 4.13) suggests, in micellar phase both trans and gauche conformations exist, while near phase transition (solid phase) the trans conformations are dominated over gauche. The peak heights are used frequently to determine the trans to gauche ratio, because estimating the integrated intensities of the peaks (area under the peak) requires the detailed peak profile analysis (theoretical fitting) and the heights of the peaks vary more strongly than integrated intensities, especially at phase transition temperature [Susi_1980, Vogel_1981]. However, the ratio of trans band $1123 \mathrm{~cm}^{-1}$ to gauche band 1140 $\mathrm{cm}^{-1}$ do not vary significantly with both methods as shown in Figure 4.13, because these band profiles are not much complicated compared to the trans $1064 \mathrm{~cm}^{-1}$ and trans $1085 \mathrm{~cm}^{-1}$. The depolarized and weakly polarized Raman bands, i.e. bands which are dominated by anisotropic component show considerable changes in their widths with temperature (see Figure 4.11). Identification of polarized and depolarized (both isotropic and anisotropic components) bands is already discussed in Chapter 3. Narrowing of the anisotropic component of Raman bands in solid phase is mainly due to decrease of molecular reorientational fluctuations [Bartoli_1972], the broad bands in micellar phase was due to the reorientational freedom of alkyl chain. The ratio of peak heights of methylene $\left(\mathrm{CH}_{2}\right) \mathrm{C}-\mathrm{H}$ asymmetric to $\mathrm{C}-\mathrm{H}$ symmetric has been extensively used to demonstrate interchain interactions/chain order disorder parameter [Gaber_1977, Wong_1983, Picquart_1986]. In Figure 4.15b a sharp decrease in ratio of asymmetric to symmetric $\mathrm{C}-\mathrm{H}$ stretching near the phase transition temperature suggests increase of interchain interaction or interchain coupling. Interchain coupling is clearly shown also in splitting of $\mathrm{CH}_{2}$ bending modes near $1450 \mathrm{~cm}^{-1}$ (Figure 4.9b).

For the odd numbered carbon atoms of n-alkanes splitting up of methylene rocking (near $724 \mathrm{~cm}^{-1}$, in alkanes) and methylene scissoring band (near 1465 $\mathrm{cm}^{-1}$ band) was observed, which is a result of intermolecular coupling, Casel et 
al. [Casel_1982] have demonstrated the splitting up of methylene rocking band by isotopic dilution methods $\left(1: 24\right.$ of $\mathrm{C}_{19} \mathrm{H}_{40}$ and $\left.\mathrm{C}_{19} \mathrm{D}_{40}\right)$ and found no splitting and they conclude that, this band should have splitted, if the splitting is not a result of intermolecular coupling. In polyethylene and $n$-alkanes, with odd numbered carbon atoms $\left(\mathrm{CH}_{2}\right.$ groups $)$ crystal structure appears to be orthorhombic, whereas in even numbered carbon atoms the crystal structure would be monoclinic or triclinic [Snyder_1961]. In such cases the orientations of the methylene chain is highly ordered with zig-zag form. The monoclinic crystal structure is similar to orthorhombic with slight tilted chains from its normal. Two molecules per unit cell were observed in monoclinic crystal structure. The interaction/coupling between these two results in the splitting up of this band. Silva et al. [Silva_2003] have recently reported the single crystal X-ray diffraction result for TTAB crystals, and found that the alkyl chain is extended in zig-zag form and packed in parallel within the layer and antiparallel in alternate layers with a monoclinic crystal structure. Our Raman spectrum near the phase transition (in solid phase) evidences the monoclinic crystal structure by showing a peak near $1417 \mathrm{~cm}^{-1}\left(\mathrm{CH}_{2}\right.$ bending in crystalline phase). Boerio and Koenig [Boerio_1970] have found the differences in the spectra of $n$-alkane and polyethylene crystals, the crystals with monoclinic structure show a peak near $1418 \mathrm{~cm}^{-1}$ at $25^{\circ} \mathrm{C}$, while crystals with triclinic and hexagonal structures do not show this peak. 


\section{Small Angle Neutron Scattering}

The formation of solid phase is observed also in Small Angle Neutron Scattering measurements. Figure 4.16 is presented the SANS intensity profiles for $300 \mathrm{mM}$ TTAB in $\mathrm{D}_{2} \mathrm{O}$, the intensity profile at $15^{\circ} \mathrm{C}$ shows a correlation peak (structure factor) characteristic of the micellar solution. The intensity decreases on cooling, at $7{ }^{\circ} \mathrm{C}$, the correlation peak disappears and intensity at lower $\mathrm{Q}$ increases (form factor), which is characteristic of solid phase. A small difference in the phase transition temperature is observed in Raman and SANS experiments where $\mathrm{H}_{2} \mathrm{O}$ and $\mathrm{D}_{2} \mathrm{O}$ are used as solvents respectively. Phase transition in case of SANS measurements is around $2-3{ }^{\circ} \mathrm{C}$ higher than the Raman measurements in agreement with the findings of Yang and Mantsch [Yang_1986] who shown in terms of Krafft temperature that surfactant with $\mathrm{D}_{2} \mathrm{O}$ have $2-4{ }^{\circ} \mathrm{C}$ higher than in $\mathrm{H}_{2} \mathrm{O}$.

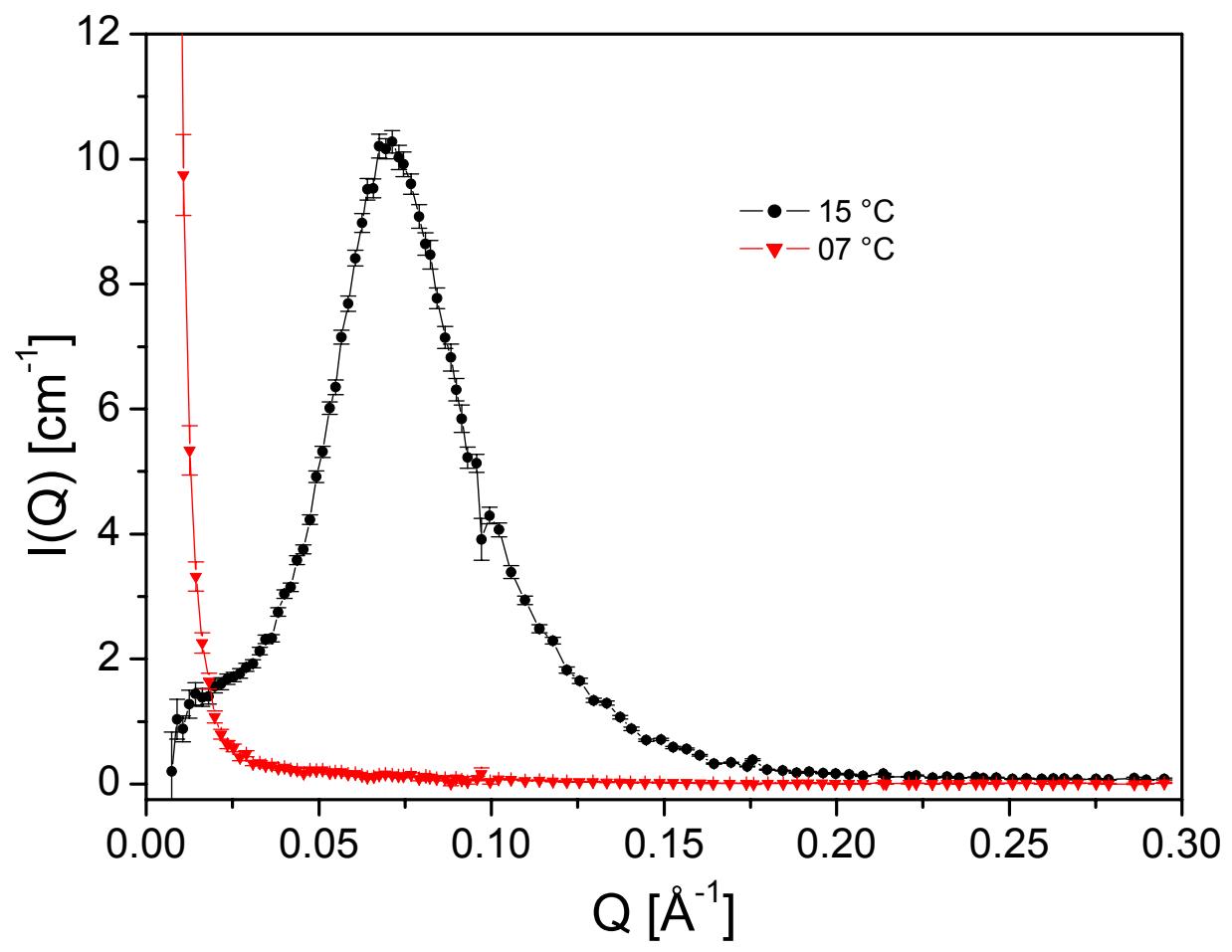

Figure 4. 16: Representative SANS intensity profiles for $300 \mathrm{mM}$ TTAB solution in $\mathrm{D}_{2} \mathrm{O}$, at $7{ }^{\circ} \mathrm{C}$ solidification observed 


\subsection{Influence of pressure}

This part of the thesis presents influence of pressure on aqueous micellar solution of TTAB. As we know already that the aggregation number and length of the micelles depends on the surfactant concentration. So, here, first influence of pressure on low surfactant concentration $(25 \mathrm{mM})$ and then high surfactant concentration $(>200 \mathrm{mM})$ is presented.

\subsubsection{Low concentration regime}

For a surfactant concentration of $50 \mathrm{mM}$ and at $25^{\circ} \mathrm{C}$, Figure 4.17 , displays Raman spectra that were obtained in the micellar phase (100 bar), during the phase transition at 1100 bar and well above the transition in the solid phase at 1300 bar and 1500 bar, respectively. For comparison, the spectrum of the dry powder of TTAB is also shown in the Figure 4.17.

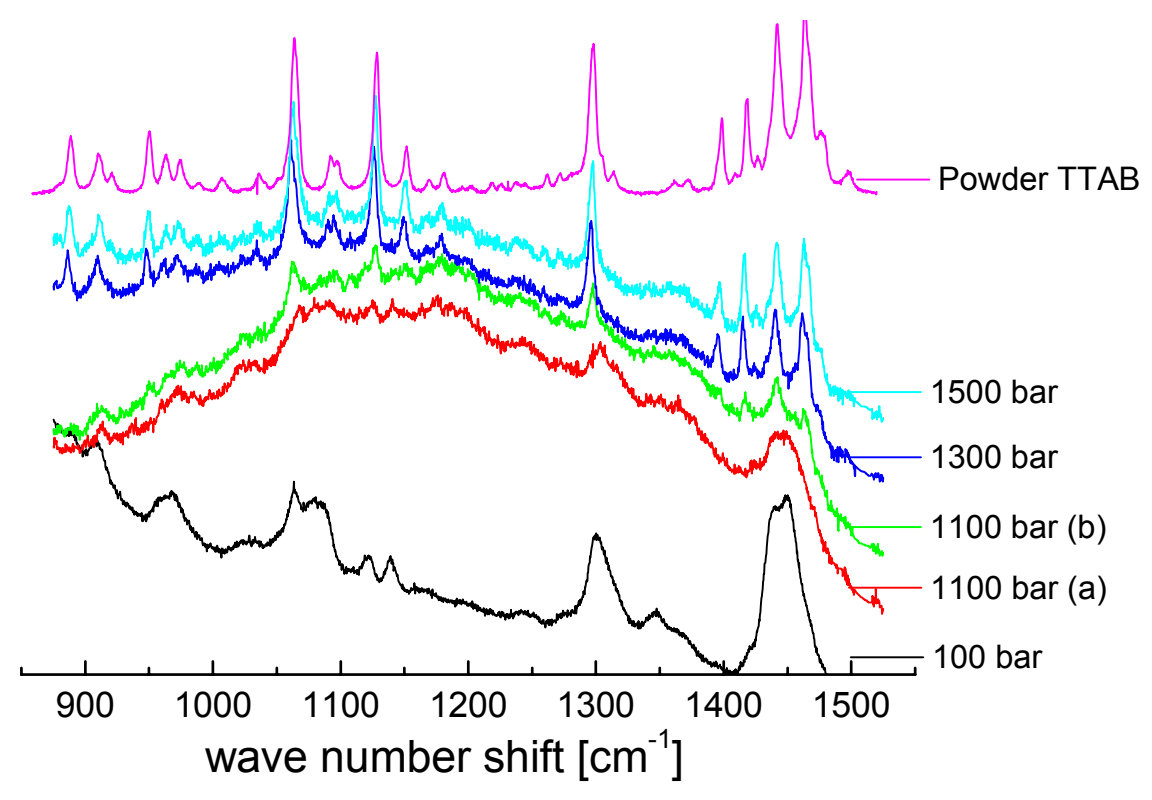

Figure 4. 17: Pressure dependence of Raman spectra for $50 \mathrm{mM}$ TTAB-solution at $25^{\circ} \mathrm{C}$ in the range of $\mathrm{C}-\mathrm{C}$ skeleton and $\mathrm{C}-\mathrm{H}$ bending vibrations. The two spectra at 1100 bar correspond to subsequent runs during phase transition. For comparison, the spectrum of dry TTAB-powder is shown in the upper part 
The spectral region showed in Figure 4.17, covers the C-C skeleton vibrations, $\mathrm{CH}_{2}$ twisting and the $\mathrm{CH}_{3} / \mathrm{CH}_{2}$-bending vibrations. The spectral features of micellar solution are already explained in the first part of this Chapter.

On increasing pressure, the spectra did not change significantly up to 1000 bar. At 1100 bar, however, the form of the spectrum starts to change drastically. It is now dominated by a broad intensity distribution extending from $1000 \mathrm{~cm}^{-1}$ up to $1500 \mathrm{~cm}^{-1}$ (spectrum a). The spectral lines have almost disappeared. After about 3 hours, a second spectrum (b) was taken at the same pressure. In the bending regime near $1450 \mathrm{~cm}^{-1}$, in particular, the evolution of sharp lines is recognised that seems to go hand in hand with the transformation into the solid phase as a result of increased intermolecular coupling/interactions. At 1300 bar and 1500 bar, the spectra exhibit the characteristic features of solids: the broad background is reduced and sharp lines are established instead. These spectra and in particular, the positions of the individual lines agree remarkably well with the results obtained from a dry powder TTAB (see upper spectrum in Figure 4.17). Moreover, the fraction of gauche isomers is strongly reduced in the solid phase as characterised by the ratio of integrated intensities of the bands at 1123 $\mathrm{cm}^{-1}$ (trans-band) and $1140 \mathrm{~cm}^{-1}$ (gauche-band), e.g. The corresponding data have been obtained by fitting the individual bands to Gaussians. The pressure dependence of the intensity ratio is shown in Figure 4.18a. For pressures up to 1000 bar, there are almost no conformational changes observed. On solidification at 1100 bar however, the trans-vibrations gain intensity on the expense of the gauche-ones. In contrast to the liquid micellar phase, the ordered arrangement of the solid phase is obviously characterised by a strong reduction of the gauche isomers. Note, that this behaviour is confirmed by the quantitative analysis of other trans-gauche bands. The peak separation of these two bands has also been used to monitor the phase transition (Figure 4.18b).

The broad and unstructured intensity distribution observed during the transition might be an indication of an intermediate gel-like state that is formed when individual micelles disaggregate and the surfactant molecules rearrange into an 
ordered crystal lattice. The high pressure phase transition from micelles to solid phase is similar to the phase transition at lower temperature at ambient pressure.
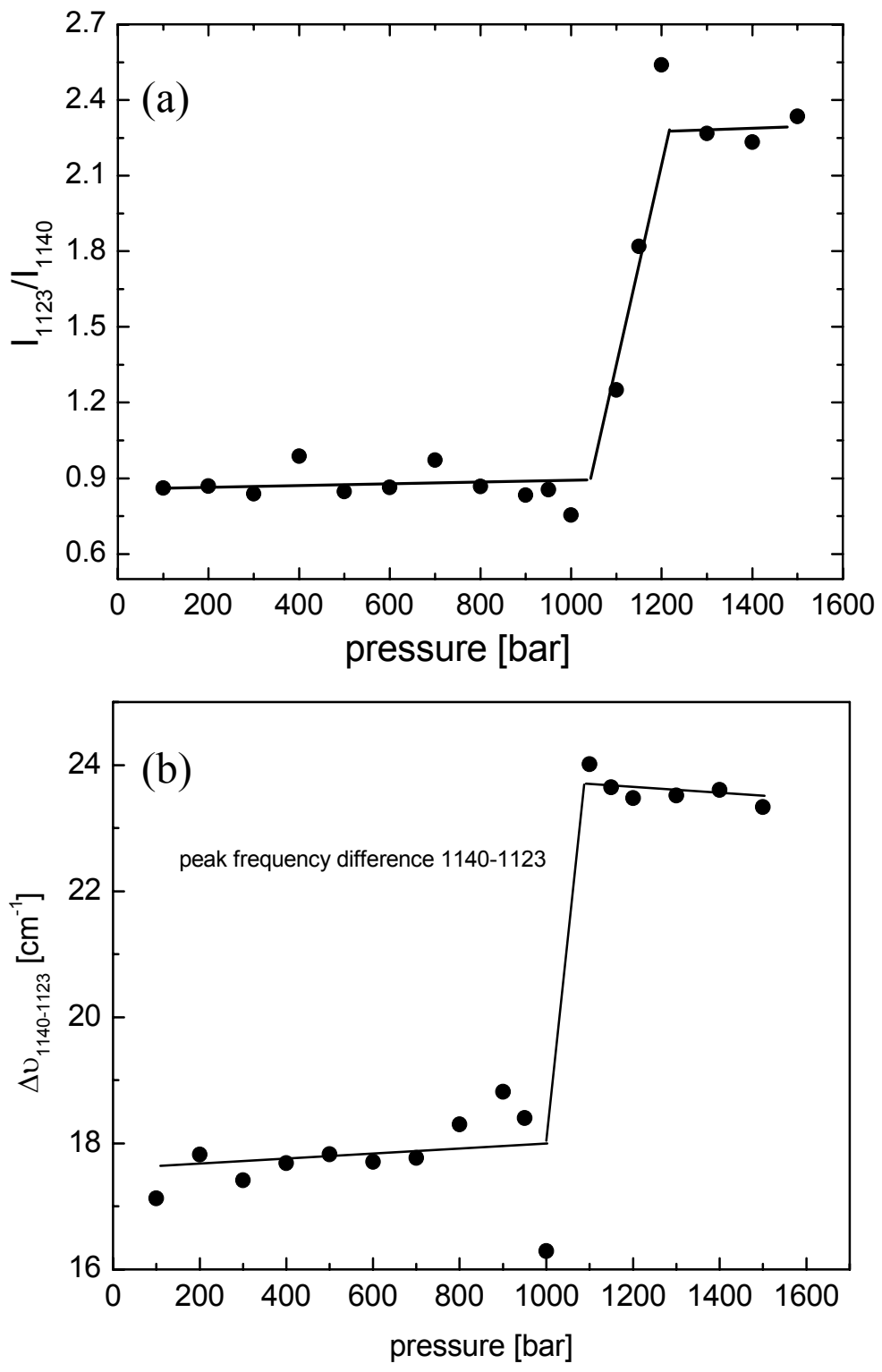

Figure 4. 18: (a) Pressure dependence of the intensity ratio of trans and gauche bands at $1123 \mathrm{~cm}^{-1}$ and $1140 \mathrm{~cm}^{-1}$ (The line is drawn to guide the eye.), (b) pressure dependence of the peak separation of trans and gauche-bands at $1123 \mathrm{~cm}^{-1}$ and $1140 \mathrm{~cm}^{-1}$ 
Pressure induced phase transitions in n-alkanes [Schoen_1979, Wong_1987] and many varieties of surfactant systems using SANS [Gorski_1997], Raman [Wong_1982, Wong_1983, Wong_1984, Wong_1986, Balzaretti_2003, Yamaguchi_2003], light scattering [Nicoli_1979] and other techniques [Offen_1980, Offen_1982, Kaneshina_1990, Baden_2002, Tanaka_1973, Fisch_1986] have been reported. Fisch et al. found a decrease of the mean hydrodynamic radius of sodium dodecylsulfate (SDS) micelles with increase of pressure while in non ionic surfactants Baden et al. recently observed the decrease of aggregation number at pressure of $2 \mathrm{kbar}$. Wong et al. have investigated the pressure induced phase transition in selected systems using Raman spectroscopy. The phase transition was well reflected by the discontinuities in the peak frequency, height ratio of trans and gauche bands and selected band widths. SANS data on tetradecyl-dimethylammonium (TDMAO) micellar system at elevated pressures are reported by Gorski et al. who pointed out particularly the pressure induced transition is rather sluggish. Such a sluggish phase transition is observed here too.

Small Angle Neutron Scattering intensity profiles of low surfactant concentration of $25 \mathrm{mM}$ at $25{ }^{\circ} \mathrm{C}$ are presented in Figure 4.19. The intensity profiles are dominated by a well defined correlation peak that is due to a pronounced interaction between the charged micelles.

When increasing the pressure up to 1000 bar, there are only very tiny shifts in intensity and peak position due to the compressibility of the $D_{2} \mathrm{O}$ (see Figure 4.19a). At elevated pressures, the particle density increases by several percent and hence, the correlation peak is slightly shifted to higher $Q$ and grows in intensity (see appendix 1 for details). The micellar structure itself remains almost unchanged. At 1100 bar, however, the intensity profile exhibits a dramatic time evolution as shown in the Figure $4.19 \mathrm{~b}$. On a time scale of hours the correlation peak decreases in intensity and the maximum shifts to smaller wave vectors. Obviously, the micelles are gradually removed from the solution and solid phase 
are formed. This is assumed to be the same phase as on cooling at ambient pressure.

(a)

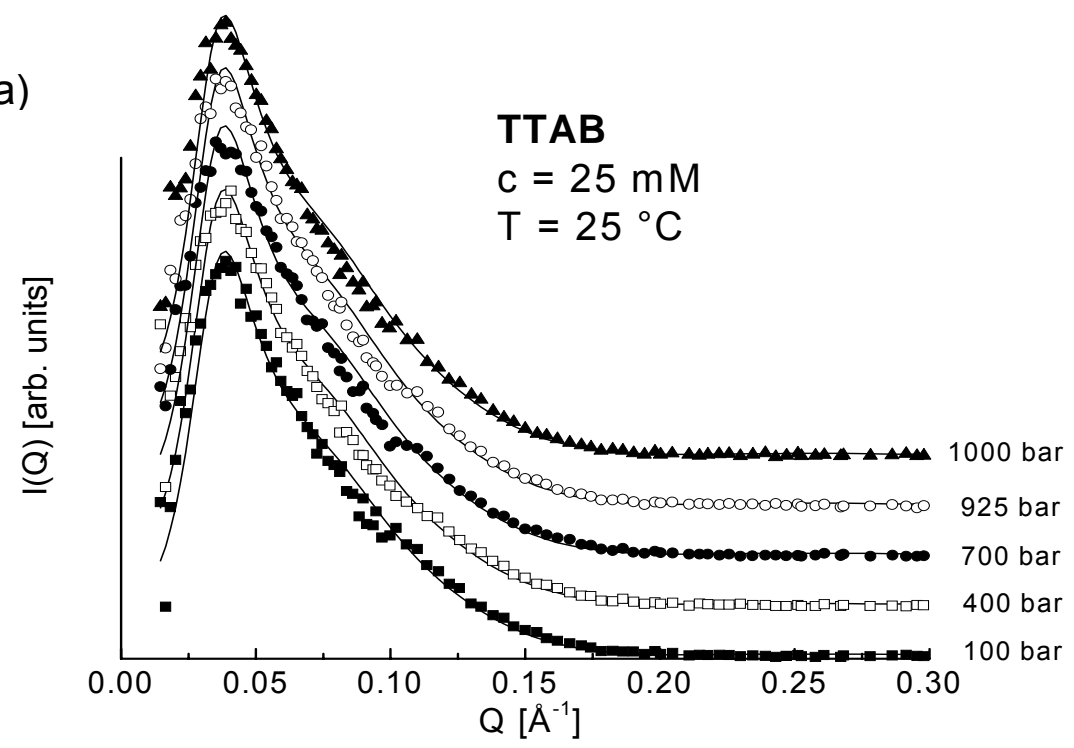

(b)

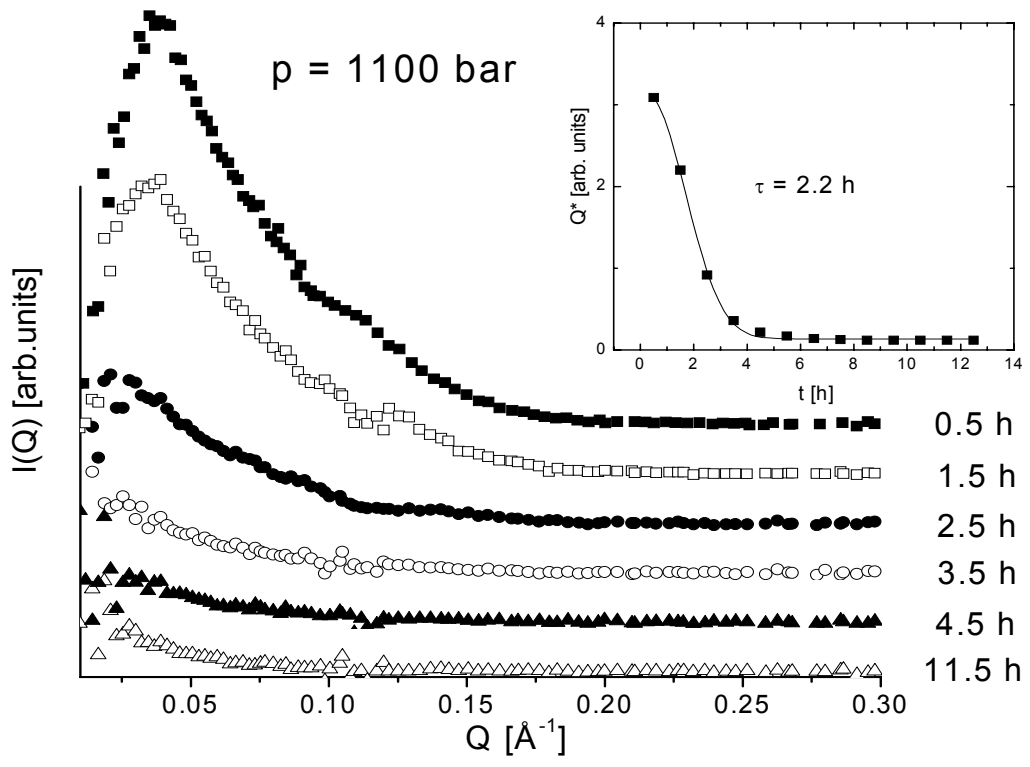

Figure 4. 19: (a) Small angle neutron scattering from $25 \mathrm{mM}$ TTAB-solution at $25^{\circ} \mathrm{C}$ at different pressures. The lines are results from a fit to the Hayter-Penfold model, (b) time evolution of scattering profiles at 1100 bar (in the inset the time dependence of the Porod invariant is shown). Note, that the individual curves are shifted vertically for clarity 
The kinetics of this solidification process can be characterized by the time dependence of the integrated intensity, i.e. the Porod-invariant:

$$
Q^{*}=\int_{0}^{\infty} q^{2} \cdot l(q) d q=2 \pi^{2} \cdot \phi \cdot(1-\phi) \cdot\left(\rho-\rho_{\text {sol }}\right)^{2}
$$

$\left(\phi, \rho\right.$ and $\rho_{\text {sol }}$ are the volume fraction and the scattering length density of micelles and the scattering length density of the solvent, respectively). The time evolution of $Q^{*}$ is shown in the inset of Figure $4.19 \mathrm{~b}$. The complete solidification $\left(\mathrm{Q}^{*}\right.$ close to zero) occurs on time scale of hours.

The Porod-invariant is well represented by a stretched exponential decay

$$
Q^{*}=\Delta Q^{*} \cdot \exp \left(-(t / \tau)^{\beta}\right)
$$

with a time constant of $\tau=(2.2 \pm 0.03)$ hours and the exponent $\beta=2.3 \pm 0.1$. The latter parameter is well within the range which is characteristic for diffusion controlled precipitation processes [Raghavan_1975]. On cooling at constant pressure, the phase transition into the solid phase can be considerably accelerated since the thermodynamic driving force for precipitation increases if the system enters more deeply into the existence regime of the solid phase.

Using the Raman and SANS experimental results of applied pressure at different temperatures a $\mathrm{p}-\mathrm{T}$ phase diagram for liquid to solid transition can be plotted (Figure 4.20). Solid and open symbols represent neutron and Raman data, respectively. It is easily recognised that both methods yield perfectly consistent results even if the surfactant concentrations are somewhat different. The isotope effect of the solvent (neutron data are obtained with $\mathrm{D}_{2} \mathrm{O}$ ) is obviously too small to be observed here. However, the difference of about $2{ }^{\circ} \mathrm{C}$ was observed on cooling experiments (from Raman measurements with $\mathrm{H}_{2} \mathrm{O}$ solvent and SANS measurements with $\mathrm{D}_{2} \mathrm{O}$ solvent). Wong et al. have also observed this isotopic effect for the system of sodium oleate in terms of critical micelle temperature (cmt) and the difference is just about $2{ }^{\circ} \mathrm{C}$. They found cmt of about $27^{\circ} \mathrm{C}$ in $\mathrm{H}_{2} \mathrm{O}$ and in $\mathrm{D}_{2} \mathrm{O}$ it is around $29{ }^{\circ} \mathrm{C}$ (Figure 4B and 6B of reference Cameron_1982). 
The difference of about $2-4{ }^{\circ} \mathrm{C}$ for an isotropic effect, with influence of temperature was also observed by Yang et al. [Yang_1986]. The slope dp/dT is obtained as $(52.6 \pm 1.5) \mathrm{bar} / \mathrm{K}$ which is in good agreement with data for TTAB system [Offen_1982]. Since the molar volumes of the liquid and the solid phases are known to differ by about $32.4 \mathrm{~cm}^{3} \mathrm{~mol}^{-1}$ [Offen_1982], the transition entropy is obtained from the Clausius-Clapeyron equation as $170 \mathrm{~J} \mathrm{~mol}^{-1} \mathrm{~K}^{-1}$. Correspondingly, the heat of transformation is $50.7 \mathrm{~kJ} \mathrm{~mol}^{-1}$ at $298 \mathrm{~K}$. Note that the data of Figure 4.20 are obtained during solidification. The reverse transition from the solid phase to the micellar solution takes place at significantly lower pressures, with hysteresis of about 400 bar. This observation is consistent with the hysteresis found on cooling and heating experiments (about $8^{\circ} \mathrm{C}$ ) at ambient pressure. So, we did not find any difference between temperature and pressure induced solidification/precipitation. The transformation behaviour is completely reproducible.

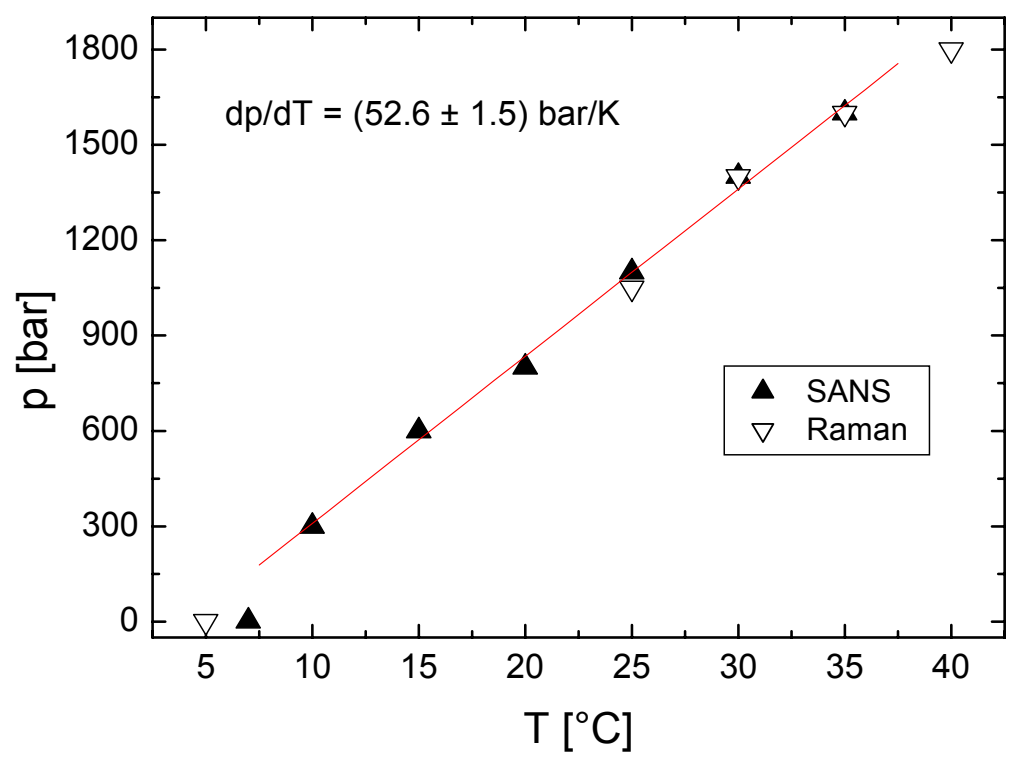

Figure 4. 20: $\mathrm{p}$-T phase diagram of TTAB solution at low concentrations $(25 \mathrm{mM} / 50$ $\mathrm{mM}$ ) as obtained by SANS and Raman scattering 


\subsubsection{High concentration regime}

Interestingly, solutions of higher surfactant concentration exhibit a transformation behaviour that differs qualitatively from the findings at $25 \mathrm{mM}$ (c.f. Figure 4.19). As an example, Figure 4.21 shows the time-evolution of small angle scattering for $360 \mathrm{mM}$ solution after increasing the pressure to 700 bar. On a time-scale of hours, the correlation peak decreases and additional intensity close to $Q=0$ corresponding to the solid precipitates appears even at this rather low pressure. A complete transformation is however, not achieved. Rather, the correlation peak is observed even for very long times (> $500 \mathrm{~min}$ ). The total intensity as characterised by the Porod invariant ' $Q$ '” (equation 3 ) approaches a saturation value that is about $1 / 3$ of the initial value as illustrated in the inset of Figure 4.21.

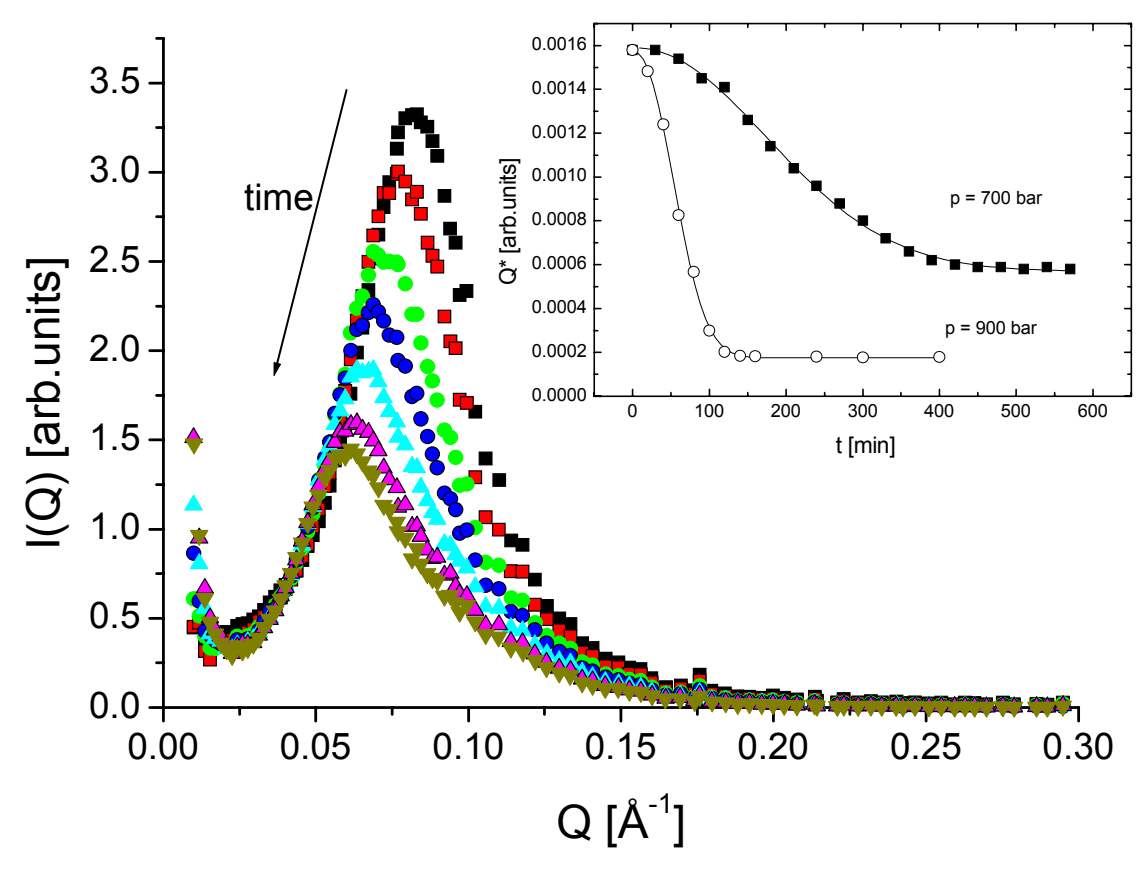

Figure 4. 21: Time evolution of SANS intensity of $360 \mathrm{mM}$ TTAB solution at $25^{\circ} \mathrm{C}$ and 700 bar. In the inset, the time dependence of the Porod invariant is shown - for comparison the data for 900 bar are also included 
As we did in low concentration regime, here again the decay of $\mathrm{Q}^{*}$ can again be well described by a stretched exponential modified, however, by a constant long time limit ' $Q_{\infty}^{*}$ '

$$
\mathrm{Q}^{*}=\mathrm{Q}^{*}{ }_{\infty}+\Delta \mathrm{Q}^{*} \cdot \exp \left(-(\mathrm{t} / \tau)^{\beta}\right)
$$

with $Q_{\infty}^{*}=(0.57 \pm 0.1) \cdot 10^{-3}, \quad \Delta Q^{*}=(1.02 \pm 0.4) \cdot 10^{-3}, \tau=(240 \pm 5)$ min and $\beta=$ (2.1 \pm 0.4$)$.

At higher pressure (900 bar), the kinetics is much faster ( $\tau=69 \mathrm{~min})$ and the residual equilibrium intensity is smaller but definitely higher than zero. These findings indicate that at higher concentrations there is a coexistence regime between micellar solution and solid phase. The equilibrium concentrations of the liquid phase can be determined quantitatively from the residual intensities ' $Q_{\infty}^{*}$ ' at different pressures. While at 700 bar, the remaining liquid micellar phase contains $150 \mathrm{mM}$, it is depleted to $40 \mathrm{mM}$ at 900 bar due to increased precipitation. At 1100 bar, we are left with the solid phase just as in the low concentration regime. Note, that with Raman scattering partial crystallisation cannot be detected since the volume fraction of the solid phase is too small and the chances to hit a crystallite within the laser focus is too small.

Combining all the experimental data, a p-c phase diagram is obtained which is presented in Figure 4.22. At $25^{\circ} \mathrm{C}$, the two-phase region extends from less than 700 bar up to 1100 bar where the transition is completed. Moreover, the Raman data suggest that in dilute solutions there might be an intermediate, gel-like state (high background) close to 1100 bar (see above). With increasing temperature, the two-phase region is shifted not only to larger pressures but also to larger concentrations. At $31^{\circ} \mathrm{C}$ and 1100 bar, e.g. the phase boundary is observed at $110 \mathrm{mM}$, while the maximum concentration of the liquid phase is reduced to 33 $\mathrm{mM}$ at $20^{\circ} \mathrm{C}$ and 700 bar. Schematically, the variation of the two-phase gap is also indicated in Figure 4.22. 


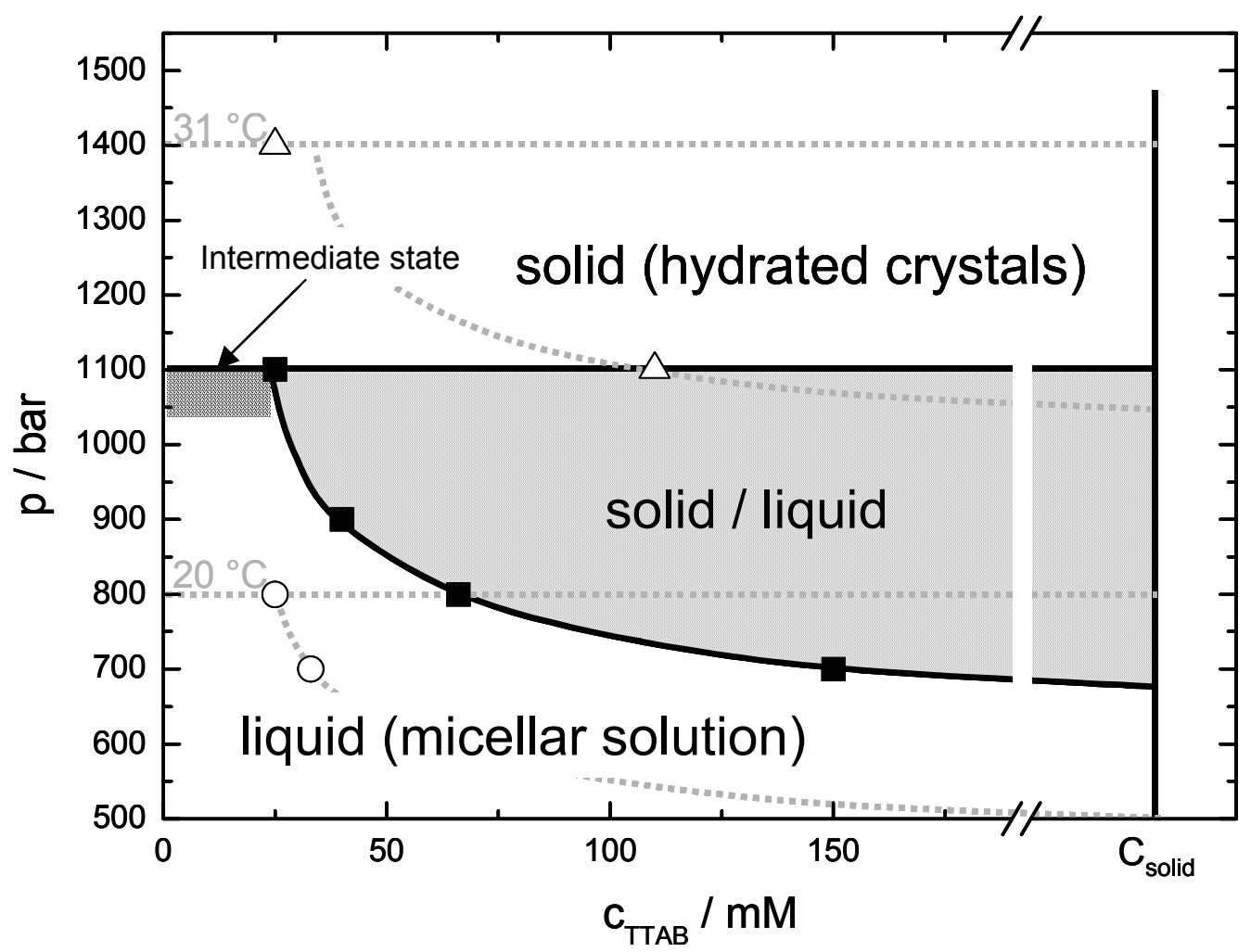

Figure 4. 22: Pressure-concentration phase diagram of TTAB at $25^{\circ} \mathrm{C}$. The temperature variation of the two phase region is schematically indicated

\subsection{Conclusion}

Using SANS and Raman spectroscopy, the phase transition in TTAB from the liquid micellar phase to solid phase has been studied over a wide range of temperature, pressure and surfactant concentration. At ambient conditions the aggregation number and length of the micelles increases with increase in surfactant concentration. At ambient pressure, on cooling the solidification occurs near $5{ }^{\circ} \mathrm{C}$ and $7{ }^{\circ} \mathrm{C}$ using Raman $\left(\mathrm{H}_{2} \mathrm{O}\right.$ solvent) and SANS $\left(\mathrm{D}_{2} \mathrm{O}\right.$ solvent) measurements respectively.

At room temperature, for the case of low concentration $(25<\mathrm{c}<100 \mathrm{mM})$ TTAB, complete solidification occurs with applied pressure of 1100 bar. The broad unstructured Raman spectrum near 1000 bar might be attributed to gel phase. 
The kinetic measurements suggest that the phase transition is found to be extremely sluggish with characteristic time scale of the order of hours. With temperature the phase transition pressure ' $p_{c}$ ' increases according to $\mathrm{dp} / \mathrm{dt}=50$ bar $\mathrm{K}^{-1}$. At higher surfactant concentration (c $>100 \mathrm{mM}$ ), a two phase regime is found at pressure below $p_{c}$ where the liquid micellar phase and the solid phase coexist. The corresponding phase diagram is obtained from a quantitative interpretation of the small angle neutron scattered intensities. 


\subsection{Influence of electrolyte}

Now, the second part of the chapter 'results and discussion' is dedicated to the influence of added electrolyte and its influence on phase transition temperature or pressure on aqueous micellar solution of TTAB, and the influence of alkyl chain length.

\subsubsection{Influence of added electrolyte on TTAB at ambient conditions;}

We know that micelles are charged particles and are spatially arranged in the solution at ambient conditions. The repulsive forces facilitate the ordering of the micelles and give rise to a correlation peak in SANS intensity profile as shown in the previous section. The importance of this section is to understand the influence of added electrolyte, $\mathrm{NaBr}$ on micellar solution of TTAB.

Raman scattering has been used to understand the influence of electrolyte on 50 mM TTAB micellar solutions, which can give information on the alkyl chain conformations with added electrolyte. For $50 \mathrm{mM}$ TTAB solution, Figure 4.23 presents Raman spectrum in $\mathrm{C}-\mathrm{C}$ stretching region. In the previous section it has been already discussed about the importance of this region. From this figure, it's very clear that the intensity of the peak at $1064 \mathrm{~cm}^{-1}$ that corresponds to the trans conformation increases with addition of electrolyte $(\mathrm{NaBr})$. In the spectrum of electrolyte free solution, the intensity of the gauche conformation band around $1085 \mathrm{~cm}^{-1}$ is higher than the intensity of the trans conformation band (horizontal dashed lines are given to follow the peak heights). With increase in electrolyte concentration the intensity of trans conformation's peak increases, however still it retains large number of gauche conformations even at large electrolyte concentration. 


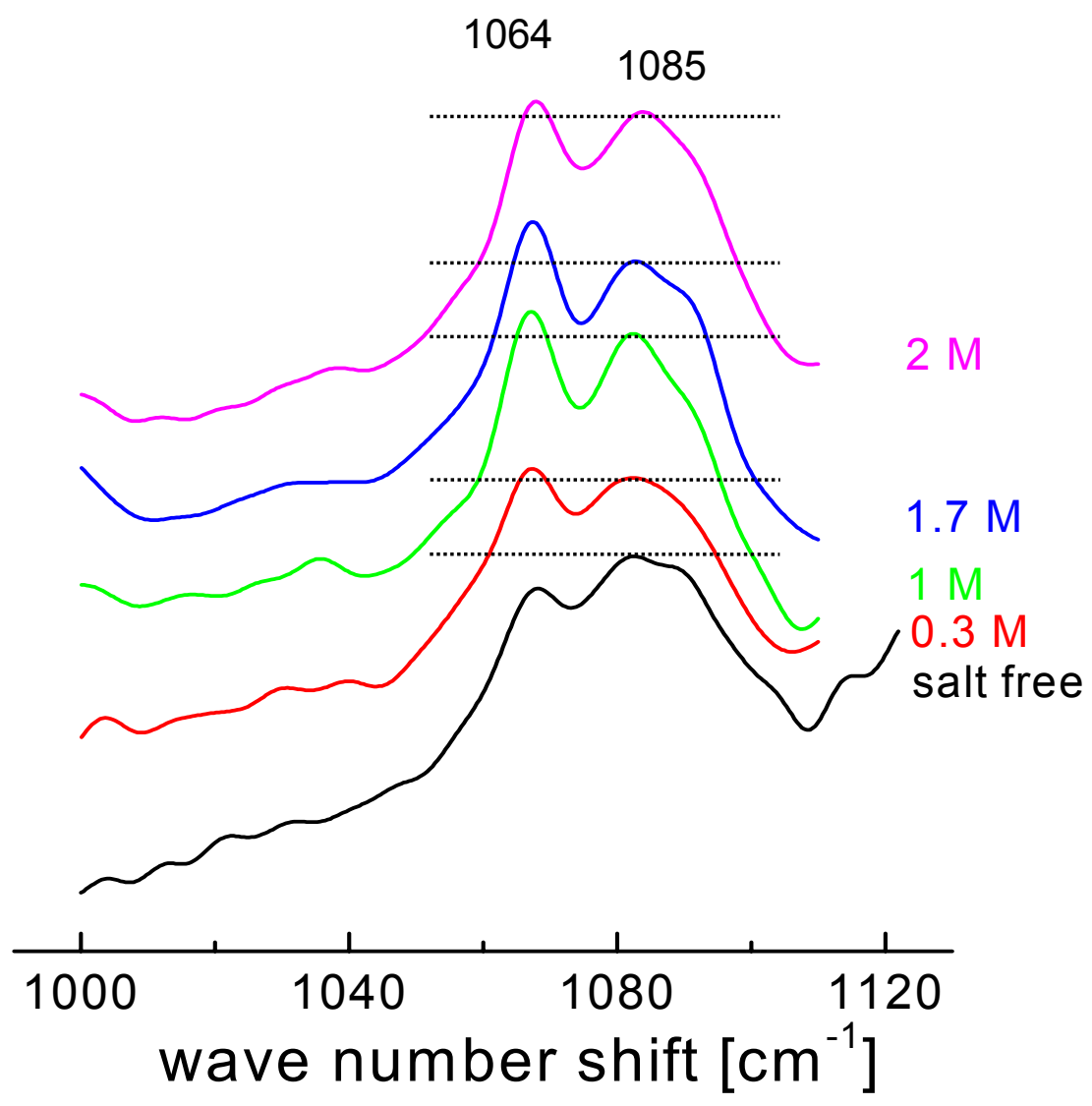

Figure 4. 23: Raman spectra of $50 \mathrm{mM}$ TTAB with and without electrolyte, $\mathrm{NaBr}$, in $\mathrm{C}-\mathrm{C}$ stretching region (the spectra are shifted vertically for clarity)

It is observed that the influence of added electrolyte is more pronounced near the head group region of the surfactant, which are present at the outer part of the micelles. Raman spectral regime from $2820 \mathrm{~cm}^{-1}$ to $3075 \mathrm{~cm}^{-1}$ for $50 \mathrm{mM}$ TTAB micellar solution with different electrolyte concentration is shown in Figure 4.23. Significant changes were observed around $3036 \mathrm{~cm}^{-1}$ vibrational band, which is assigned to $\mathrm{C}-\mathrm{H}$ stretching of $\mathrm{CH}_{3}$ group present at the head group of surfactant molecule [Kawai_1985, Hattori_1999]. This band is observable both, in zz and zy polarized spectrum. The spectra presented here are in zy polarization mode, which are aimed to get information also about the change in asymmetric methylene $\mathrm{C}-\mathrm{H}$ stretching band $\left(2889 \mathrm{~cm}^{-1}\right)$ with the addition of $\mathrm{NaBr}$. The spectrum gives qualitative information that the peak frequency shifts towards 
lower wave number in both asymmetric methylene $\left(2889 \mathrm{~cm}^{-1}\right) \mathrm{C}-\mathrm{H}$ stretching and $\mathrm{C}-\mathrm{H}$ stretching $\left(3036 \mathrm{~cm}^{-1}\right)$ of $\mathrm{CH}_{3}$ group present at the head group. Figure 4.24 presents the shift in peak frequency $\left(3036 \mathrm{~cm}^{-1}\right)$ as a function of $\mathrm{NaBr}$ concentration. Data presented in Figure 4.25 are extracted from both, the zy and zz polarized Raman spectra of $50 \mathrm{mM}$ and $400 \mathrm{mM}$ TTAB micellar solutions with added electrolytes respectively. One data point of zy polarized spectrum at $2 \mathrm{M}$ $\mathrm{NaBr}$ is not in the trend, but the asymmetric $\mathrm{C}-\mathrm{H}$ stretching band do follow the trend (see peak position around $2889 \mathrm{~cm}^{-1}$ in Figure 4.23). At this stage there is no clear information about this behavior of $3036 \mathrm{~cm}^{-1}$ peak at $2 \mathrm{M} \mathrm{NaBr}$.

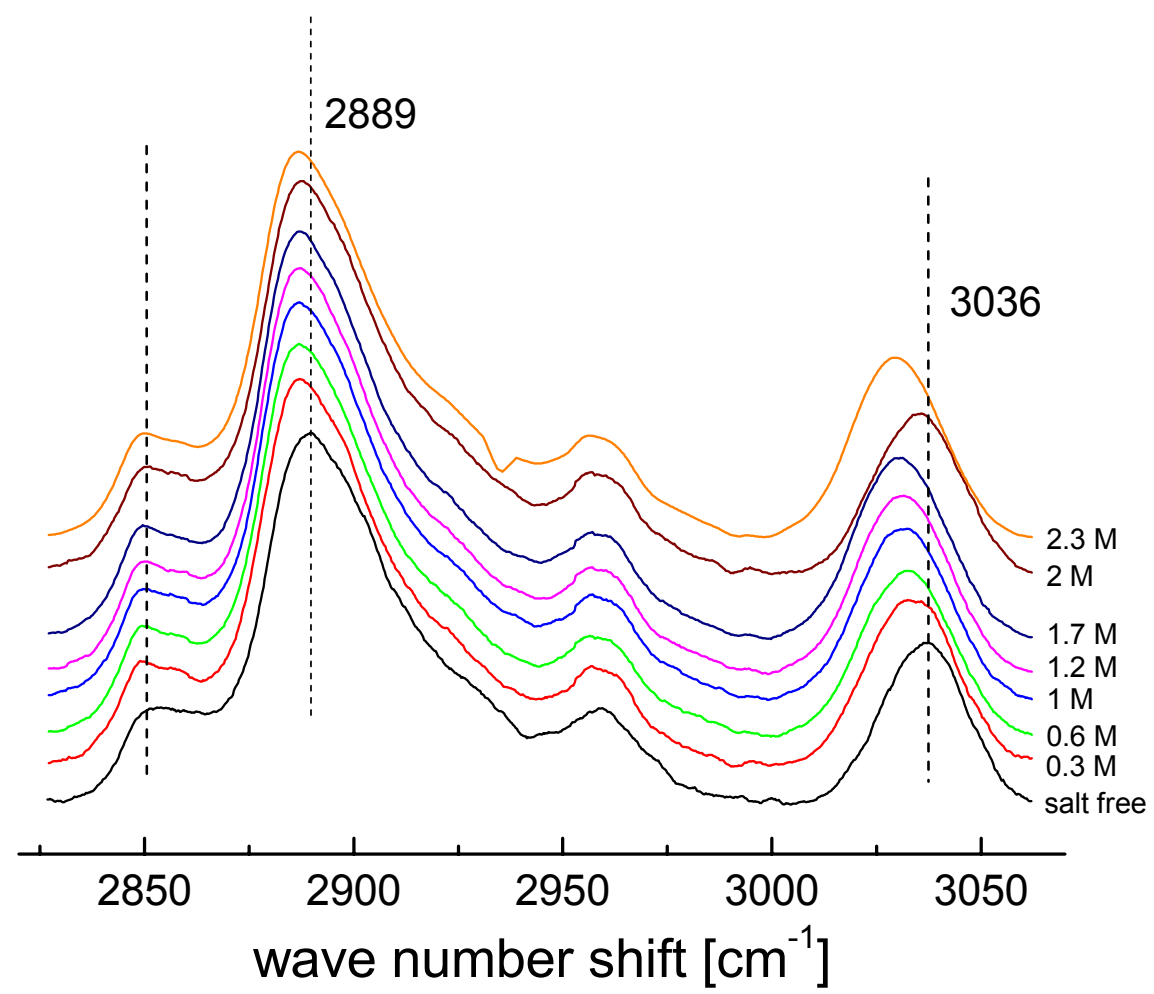

Figure 4. 24: Raman spectra in $\mathrm{C}-\mathrm{H}$ stretching region of methylene, and methyl groups of head group. Dashed vertical lines are given to follow the peak position (spectrums are shifted vertically for clarity) 


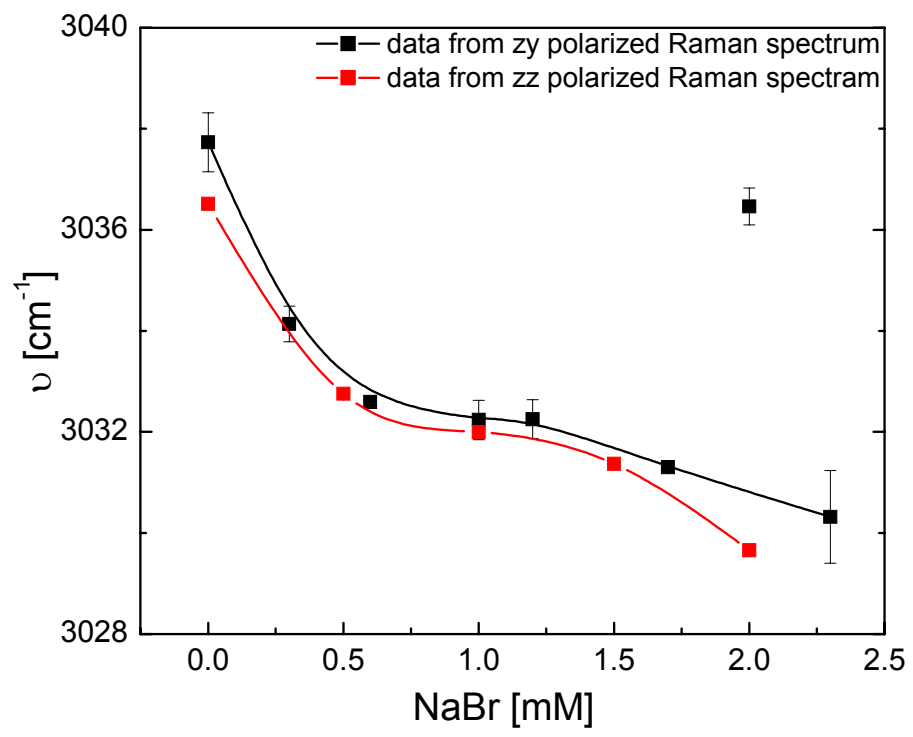

Figure 4. 25: Shift in peak frequency $\left(3036 \mathrm{~cm}^{-1}\right)$ with addition of electrolyte (the deviation of a data point at $2 \mathrm{M}(\mathrm{zy})$ might be an artifact of the experiment)

The changes in the $\mathrm{C}-\mathrm{H}$ stretching region on addition of different electrolyte, like $\mathrm{NaBr}, \mathrm{NaCl}$ and $\mathrm{NaNO}_{3}$ lead the same behavior as shown by Amorim [Amorim_1982]. They followed the ratio of peak height of asymmetric $\left(2880 \mathrm{~cm}^{-1}\right)$ and symmetric $\left(2850 \mathrm{~cm}^{-1}\right) \mathrm{C}-\mathrm{H}$ stretching vibration which is considered as order disorder parameters, and they found a slight increase with increase in the addition of salt.

The influence of $\mathrm{NaBr}$ on aqueous micellar solution of TTAB at ambient conditions is well reflected in the SANS intensity profiles too. Figure 4.26 presents SANS intensity profiles of $50 \mathrm{mM}$ TTAB with addition of electrolyte, $\mathrm{NaBr}$. SANS intensity profile of electrolyte free TTAB solution is dominated with the correlation peak originated by inter particle structure factor. The addition of $\mathrm{NaBr}$ dramatically alters the intensity profile. This correlation peak around $\mathrm{Q}_{\max }=$ $0.046 \AA^{-1}$ is gradually reduced as the amount of electrolyte is increased. At $\mathrm{NaBr}$ concentration of $100 \mathrm{mM}$ this peak has already disappeared and the intensity profile is almost entirely determined by form factor of the micelles. This finding is due to the fact that the added $\mathrm{NaBr}$ reduces the intermicellar electrostatic 
interactions substantially and hence the structure factor is reduced to unity. On further increase of electrolyte concentration, the scattered intensity grows remarkably, in particularly at lower momentum transfer (lower $Q$ value).

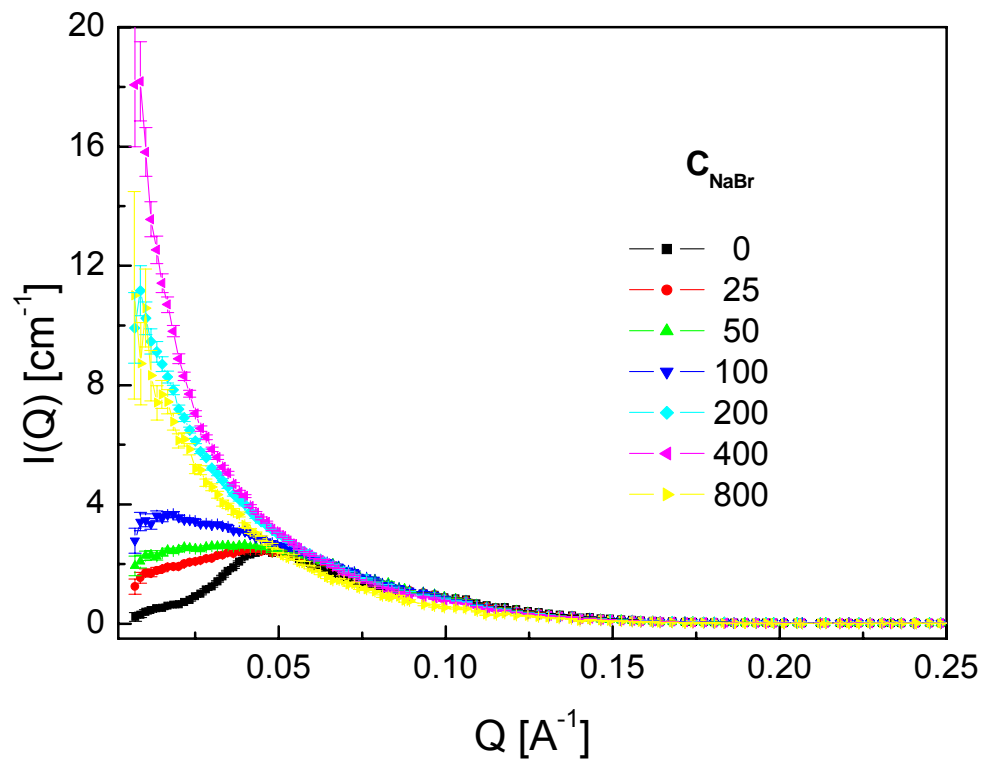

Figure 4. 26: Variation of small angle scattering intensity profiles of a $50 \mathrm{mM}$ TTAB solution with increasing $\mathrm{NaBr}$ concentration at $25{ }^{\circ} \mathrm{C}$

This behavior is a result of the growth of micelles as a result of reduced repulsions. Since the fluid like cores of the micelles remain compact, an isotropic growth is however, not possible. Hence, the form of the micelles changes from spherical either to rod-like or to disc-like objects. A detailed analysis of the determination of the sphere to rod like micelles is described elsewhere [Eckold_2001, Imae_1986]. Static light scattering studies of alkyltrimethylammonium halide studies [Imae_1986] shows that the rod like micelles were formed by the addition of $0.12 \mathrm{M} \mathrm{NaBr}$ to TTAB micellar solutions, which is in good agreement with present findings. The radius of the micelles increases from $21 \AA$ to $25 \AA$ and the increase in the apparent surfactant volume from $415 \AA^{3}$ to $550 \AA^{3}$ was observed with increase in electrolyte concentration (from 0 to $0.4 \mathrm{M} \mathrm{NaBr}$ ), which might be a result of the dehydration of head group of the micelles [Eckold_2001]. 


\subsubsection{Influence of added electrolyte, on phase transition; with applied pressure}

It is well understood from the SANS investigation that, at ambient conditions the micellar length increases from $21 \AA$ to $25 \AA$ and the interior of the micelles become rigid with increase of electrolyte concentration [Eckold_2001]. The next problem was to understand to what extent this effect will affect the phase transition (transition from micellar phase to solid phase) pressure or temperature. Here it is presented the effect of added electrolyte on phase transition pressure of micellar solution of $50 \mathrm{mM}$ TTAB at room temperature $\left(25^{\circ} \mathrm{C}\right)$. For the electrolyte free micellar solution of $50 \mathrm{mM}$ TTAB is already discussed in Section 4.4. The phase transition is sluggish; it appears in the time scale of hours (Figure 4.17). Similar type of phase transition is also observed for electrolyte added micellar solution of $50 \mathrm{mM}$ TTAB. Raman spectra for $50 \mathrm{mM}$ TTAB with $100 \mathrm{mM}$ electrolyte $\mathrm{NaBr}$ is presented in Figure 4.27.

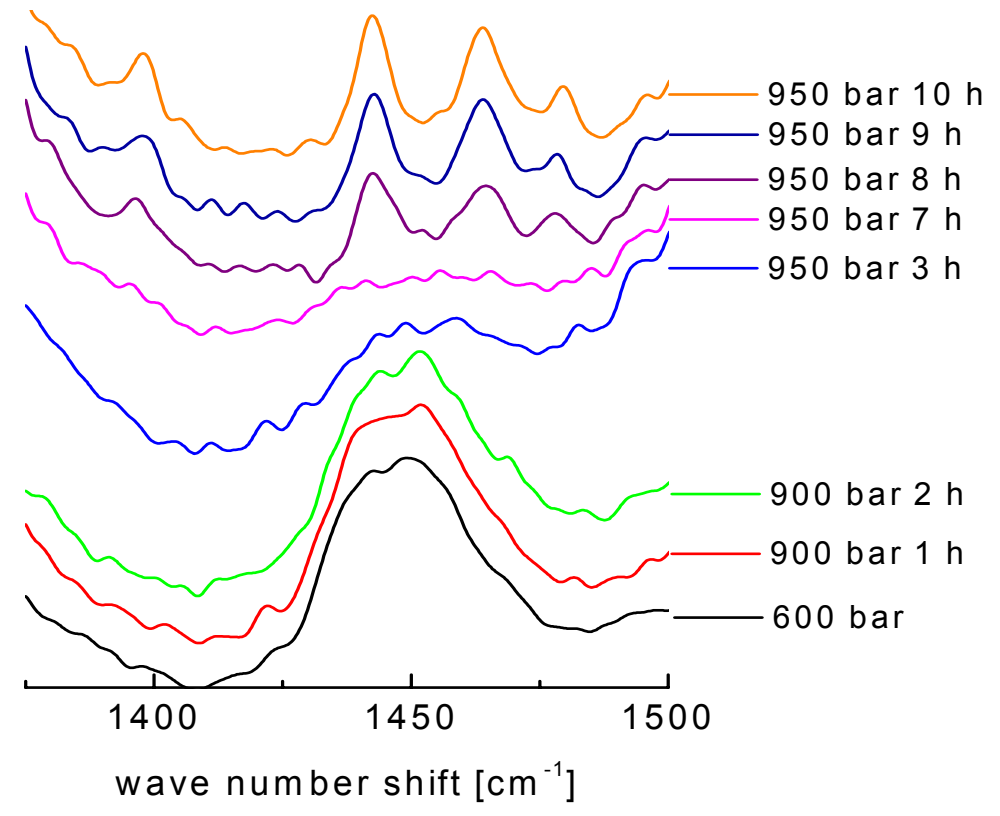

Figure 4. 27: Evolution of Raman spectra in the region of $\mathrm{CH}_{3} / \mathrm{CH}_{2}$ bending vibrations at $25^{\circ} \mathrm{C}$ during crystallization at about 950 bar. (Note that the individual spectra are shifted in vertically for clarity.)

The spectral regime corresponds to $\mathrm{CH}_{2} / \mathrm{CH}_{3}$ bending modes. The narrowing of vibrational bands appears above the transition pressure 950 bar, which is 
characteristic of crystallized phase (here again the phase transition is quite sluggish and appears in time scale of hours), where all trans conformations are dominated. The phase transition pressure is significantly less than the phase transition pressure of electrolyte free system.

SANS intensity profiles also confirms the reduced phase transition pressure with added electrolyte. Figure 4.28 shows the time evolution of SANS intensity profiles for micellar solution of $50 \mathrm{mM}$ TTAB with $100 \mathrm{mM}$ added electrolyte at 1000 bar. There was no significant change in intensity profiles until it reaches the phase transition pressure, as has been stated in salt free system. Here, at 1000 bar the solidification leads to a decrease of scattered intensity in the Q-range above $0.02 \AA^{-1}$ and to an increase at very small $Q$ due to the formation of large crystalline particles.

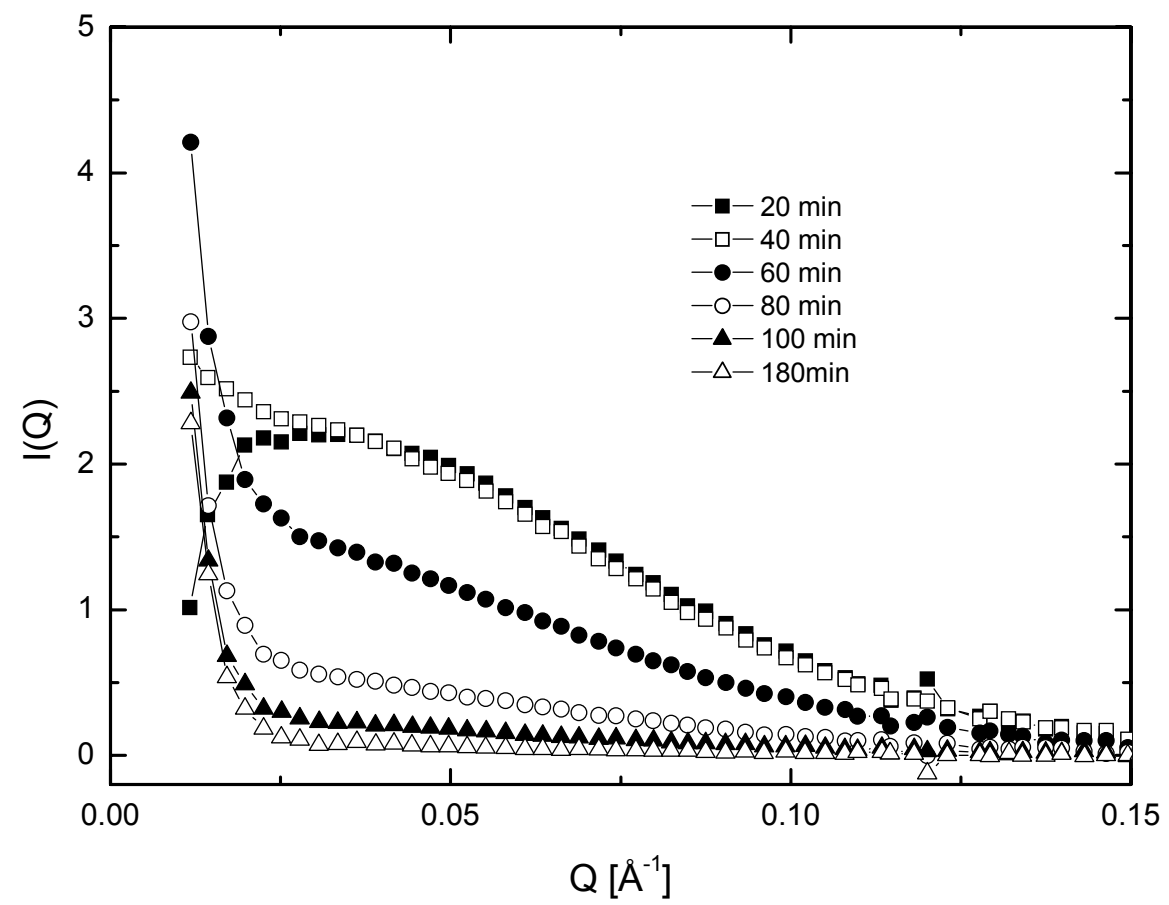

Figure 4. 28: Time-evolution of small angle scattering of 50mM-TTAB with $0.1 \mathrm{M}$ $\mathrm{NaBr}$ at $30^{\circ} \mathrm{C}$ after increasing the pressure to 1000 bar 
The measurements have been done at different temperatures. In Figure 4.29 pressure temperature phase diagram for surfactant system with added electrolyte is presented in comparison with electrolyte free surfactant system. In presence of electrolyte the phase transition pressure was significantly reduced in comparison with electrolyte free system. Both, Raman and SANS techniques provide consistent results in terms of phase transition pressure. The slope $\mathrm{dp} / \mathrm{dT}$ of about $53 \mathrm{bar} / \mathrm{K}$ is observed for electrolyte free solutions at small surfactant concentrations (Figure 4.20). The present results with added electrolyte show that there is a shift of the liquid-solid phase boundary towards higher temperature or lower pressures. The slope however remains the same.

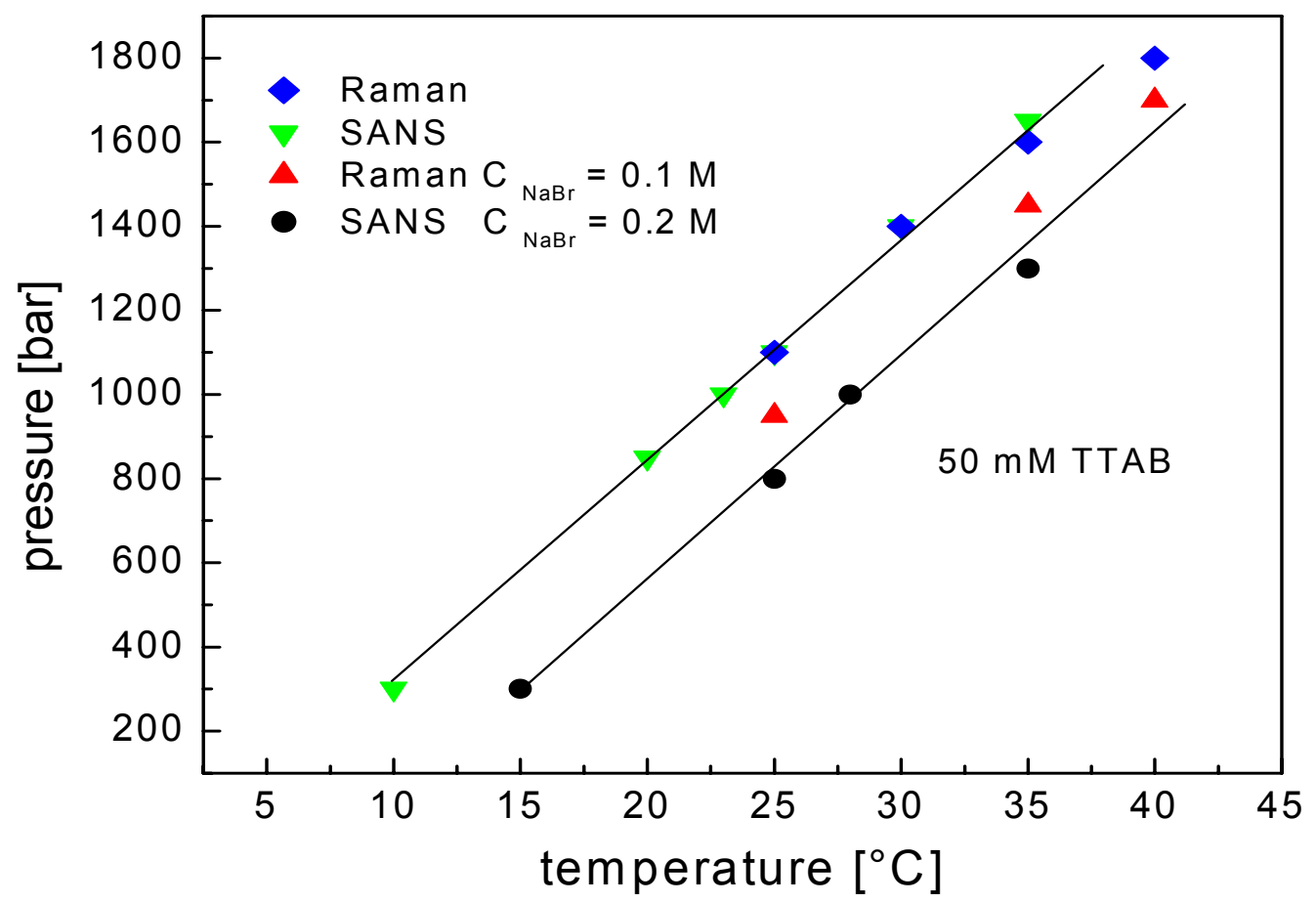

Figure 4. 29: Pressure-temperature phase diagram of $50 \mathrm{mM}$ TTAB with different concentrations of $\mathrm{NaBr}$ 


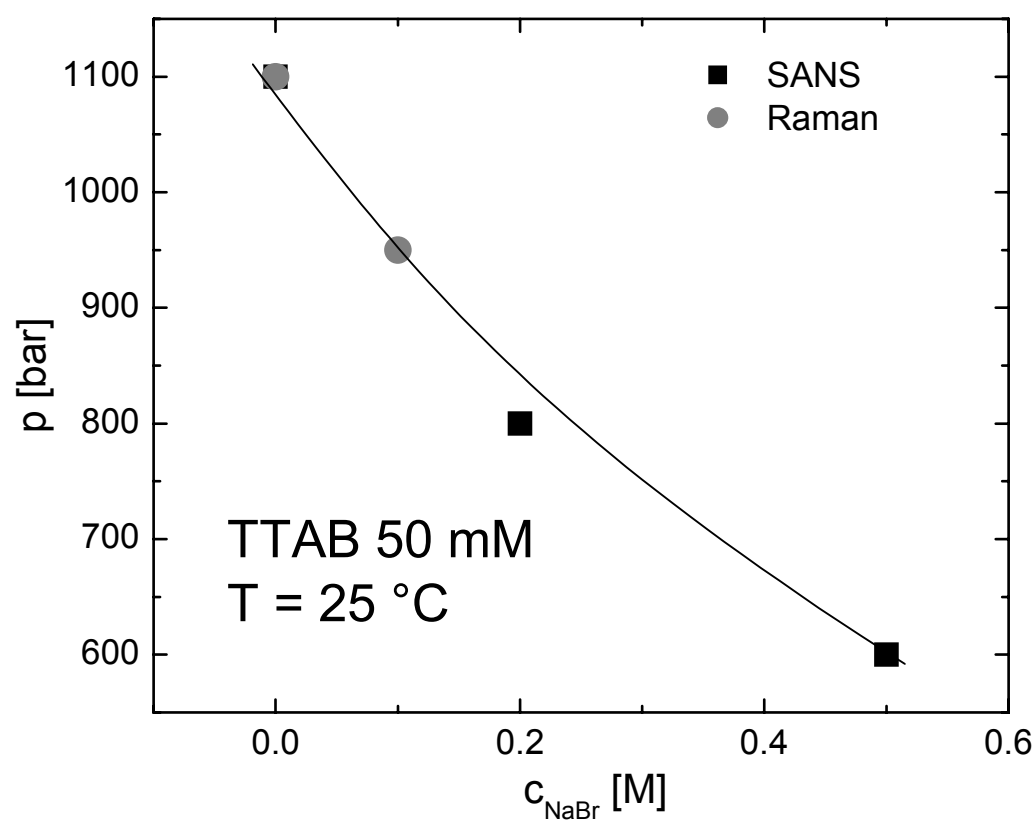

Figure 4. 30: Influence of electrolyte; variation of phase transition pressure at $25^{\circ} \mathrm{C}$ Influence of electrolyte concentration on phase transition pressure at $25{ }^{\circ} \mathrm{C}$ is presented in Figure 4.30.

One can attempt to determine the influence of electrolyte on activity coefficient of surfactant molecule in solution. From the Figure 4.30, the initial slope $\left(\mathrm{dp} / \mathrm{dc}_{\mathrm{NaBr}}=\right.$ -1500 bar $\left(\mathrm{mol}^{-1}\right.$ ) can be used to estimate the influence of the electrolyte concentration on the activity coefficient of surfactant molecules in solution. Similar to the Clausius-Clapeyron equation, $\mathrm{dp} / \mathrm{dc}_{\mathrm{NaBr}}$ can be written in the following form (the derivation of this equation is given in appendix 2):

$$
\left.\frac{\mathrm{dp}}{\mathrm{dc}_{\mathrm{NaBr}}}\right|_{\mathrm{T}=25^{\circ} \mathrm{C}}=-\left.\frac{\mathrm{RT}}{\Delta \overline{\mathrm{V}}} \frac{\partial \ln \gamma}{\partial \mathrm{c}_{\mathrm{NaBr}}}\right|_{\mathrm{T}, \mathrm{p}}
$$

Using the value of $32.4 \mathrm{~cm}^{3} \mathrm{~mol}^{-1}$ for the partial volume change [Offen_1982] during crystallization, a derivative $\partial \mathrm{ln} \gamma / \partial \mathrm{c}_{\mathrm{NaBr}}$ of $2.01 \mathrm{I} \mathrm{mol}^{-1}$ is obtained. Hence, the activity coefficient changes by about $20 \%$ if $0.1 \mathrm{~mol}$ of $\mathrm{NaBr}$ is added to the solution. 


\subsubsection{Influence of added electrolyte, on phase transition; at ambient pressure}

In section 4.3, it was discussed that, the electrolyte free 0.4 M TTAB shows the phase transition from micelles to solid phase at around $5{ }^{\circ} \mathrm{C}$. Figure 4.31 presents the influence of electrolyte on the phase transition temperature (at ambient pressure) for $0.4 \mathrm{M}$ TTAB micellar solutions. Higher surfactant concentration was chosen because in the temperature scale of $2{ }^{\circ} \mathrm{C}$ to $25^{\circ} \mathrm{C}$, the phase transition from micelles to solid phase can be achieved without disturbing the system, in other words without inducing the precipitation (nucleation), whereas low surfactant concentrations $(<100)$ requires nucleation either by shaking or stirring. The phase transition temperature increases with increase of electrolyte concentration.

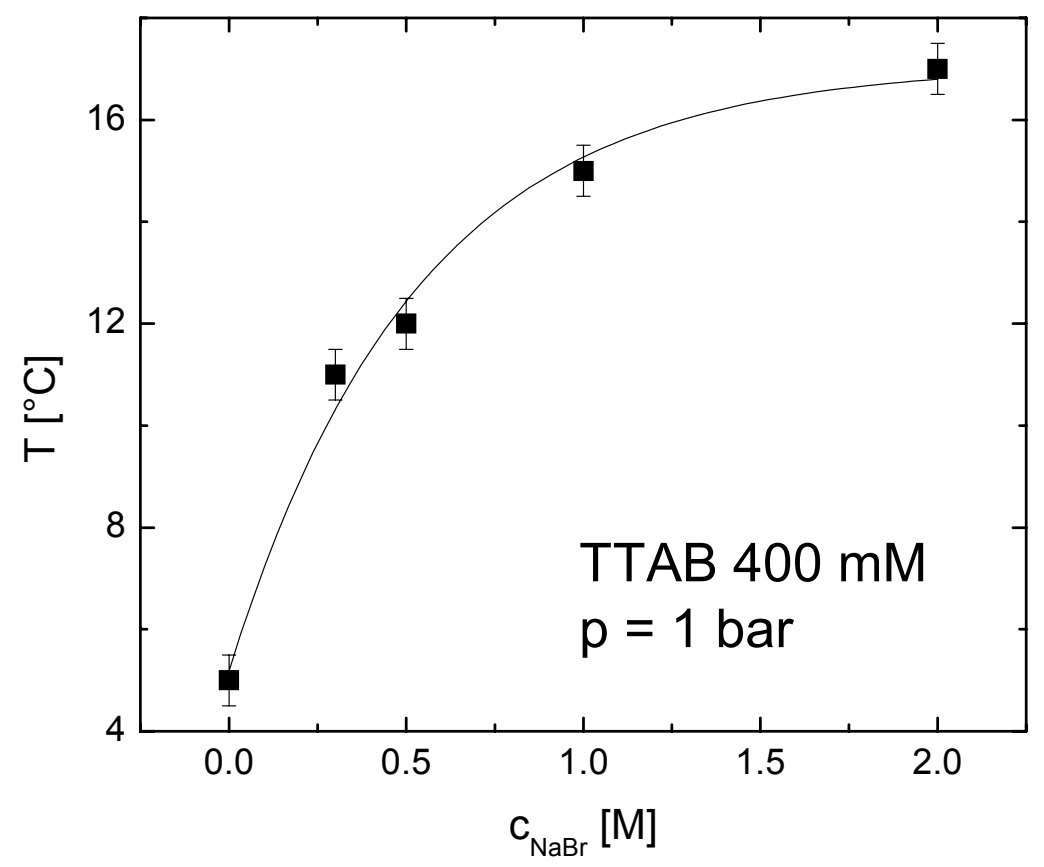

Figure 4. 31: Variation of crystallization temperature at 1 bar as a function of $\mathrm{NaBr}$ concentration

From all the above data, we can understand that the salt has significant influence on structure and dynamics of the micellar solution which finally influence the 
phase transition from micellar phase to solid phase at lower pressure or at higher temperature. This result is supported by the findings of Mazer [Mazer_1976], who showed the solid to liquid phase transition of SDS with addition of $\mathrm{NaCl}$, where the critical micellization temperature $(\mathrm{cmt})$ increases with increase of electrolyte $\mathrm{NaCl}$ concentration. 


\subsection{Influence of alkyl chain length at ambient conditions}

Experimental results of Raman and SANS for alkyl chain length ranging from $\mathrm{C} 12$ to $\mathrm{C} 18$ is presented here. The thermodynamic properties of micellar solutions show a regular trend with increase in alkyl chain length [Woolley_1985]. It is already mentioned in second Chapter that critical micelle concentration (cmc) depends on the alkyl chain length and decreases with increase of alkyl chain length [Shinoda_1963]. Phase behavior of micellar solution of these surfactants show many varieties of phases with concentration, temperature and pressure [Laughlin_1994, Rubingh_1990].

Table 4.3 presents the $\mathrm{cmc}$ for alkyltrimethylammonium bromides obtained by conductivity measurements. The cmc values for DTAB and TTAB are in good agreement with literature [Yagui_2005, Gharibi_1998], cmc of HTAB and OTAB are taken from the literature [Kalyanasudaram_1976, Gharibi_1998].

\begin{tabular}{|c|c|c|c|c|}
\hline Surfactant & DTAB & TTAB & HTAB & OTAB \\
\hline cmc & $\begin{array}{c}\text { (this work) 15.6 } \\
\text { (literature) 15.9 }\end{array}$ & $\begin{array}{c}\text { (this work) 3.8 } \\
\text { (literature) 3.9 }\end{array}$ & 0.92 & 0.3 \\
\hline
\end{tabular}

Table 4. 3: cmc of alkyltrimethyammonium bromides, for HTAB and OTAB the data are from the references [Kalyanasundaram_1976] and [Gharibi_1998] respectively

\subsubsection{Raman spectra of dry powder samples}

Before presenting the Raman spectra of micellar solutions spectra of powder sample and the change in the spectral features with alkyl chain length are presented. Figure 4.32a presents the spectra of powder sample measured at 25 ${ }^{\circ} \mathrm{C}$. The spectral region exhibits bands near $150 \mathrm{~cm}^{-1}, 450 \mathrm{~cm}^{-1}, 760 \mathrm{~cm}^{-1}$ and $900 \mathrm{~cm}^{-1}$ (shown in Figure 9a) are assigned to longitudinal accordion modes, C-N stretching, $\mathrm{CH}_{3}$ rocking from head group region $\left(\mathrm{C}-\mathrm{N}^{+}\left(\mathrm{CH}_{3}\right)_{3}\right)$, and $\mathrm{CH}_{3}$ rocking modes from terminal methyl group respectively. 

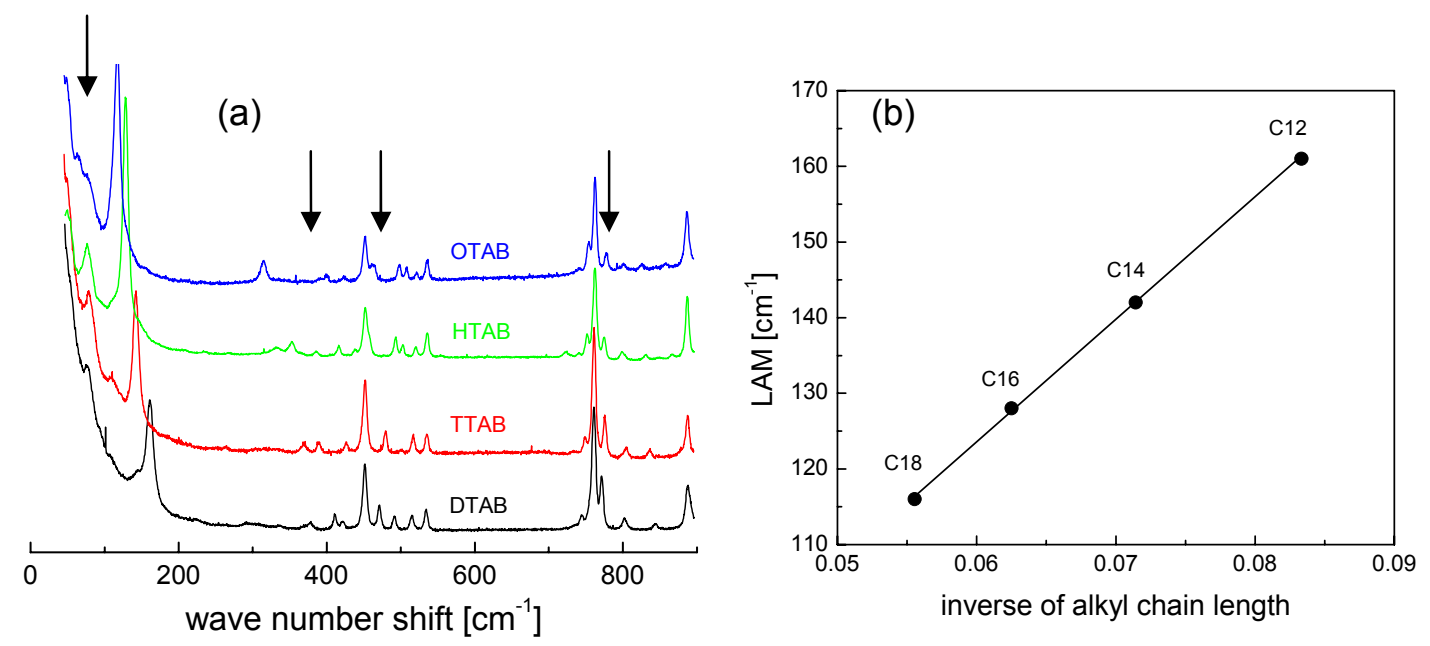

Figure 4. 32: (a) Comparison of Raman spectra for powder sample at low frequency regime (LAM), (b) the shift in peak frequency LAM with alkyl chain length. (Note that the individual spectra are shifted vertically for clarity.)

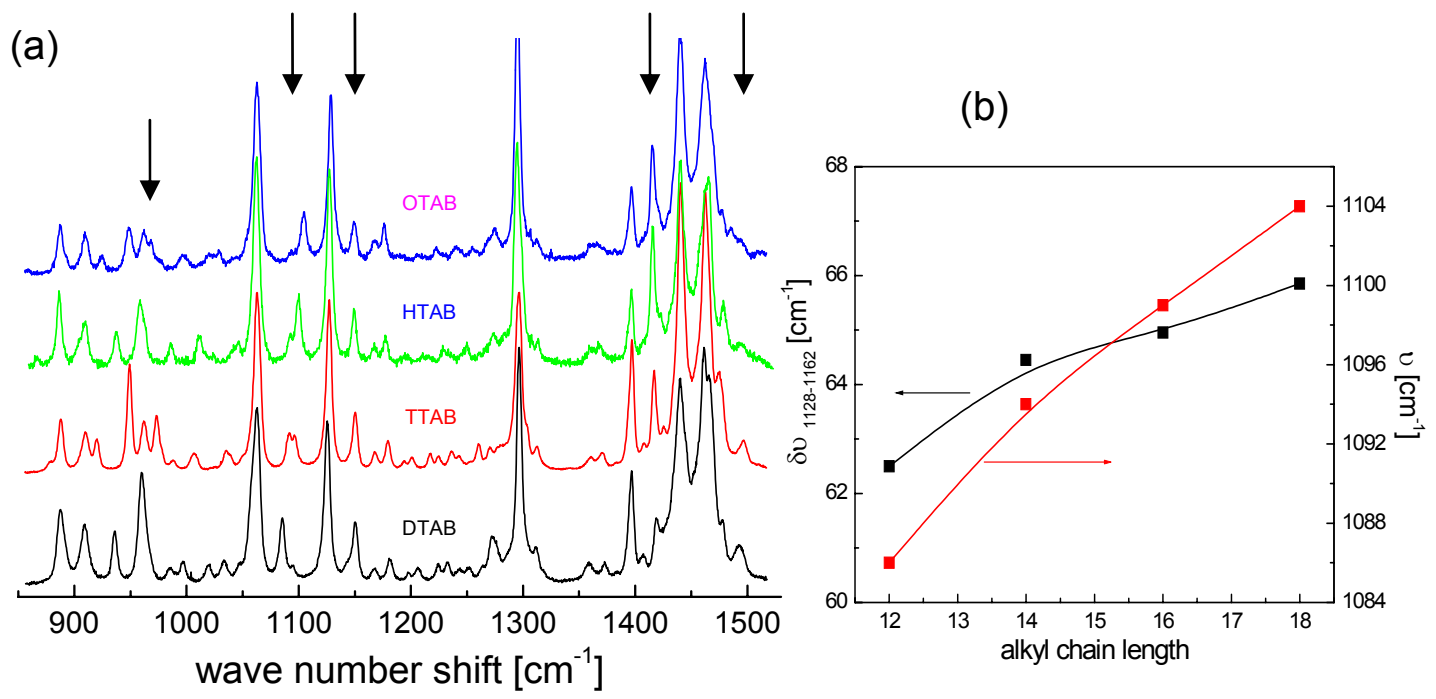

Figure 4. 33: (a) Comparison of Raman spectra for powder sample, (b) the peak frequency difference of symmetric and asymmetric $\mathrm{C}-\mathrm{C}$ stretching and shift in gauche band $\left(1090 \mathrm{~cm}^{-1}\right)$. (Note that the individual spectra are shifted vertically for clarity.)

With increasing alkyl chain length significant differences were observed in frequency and the intensity of the bands (see the arrow in Figure 4.32a). The 
striking difference was observed in the low frequency regime, particularly, at longitudinal accordian modes (LAM) near $100 \mathrm{~cm}^{-1}$ to $200 \mathrm{~cm}^{-1}$ is shown in Figure 4.32b plotted against inverse of alkyl chain length.

The peak frequency decreases from $161 \mathrm{~cm}^{-1}$ to $116 \mathrm{~cm}^{-1}$ with increase of alkyl chain length, showing linear behavior. For the alkyltrimethylammonium bromides (C6 to C12) LAM at lowest frequency decreases from $251 \mathrm{~cm}^{-1}$ to $163 \mathrm{~cm}^{-1}$ [Hattori_1999]. Similar observation was made by Mizushima [Mizushima_1949] for the Raman spectra of n-paraffins (C4 to C16). They found only one Raman peak at low frequency regime for each solid paraffins and its frequency is inversely proportional to the number of carbon atoms. This would be accounted for by approximating the extended carbon chain as a continuous rod and assigning the Raman line to its longitudinal motion, the frequency is given by the relation;

$$
v=\frac{1}{2 l} \sqrt{\frac{E}{\rho}}
$$

where ' $E$ ' is the Young's modules, ' $\rho$ ' is the density and 'l' the length of the rod. This relation implies that the frequency is inversely proportional to the length of the hydrocarbon chain.

Figure 4.33a presents Raman spectra which correspond to C-C stretching, $\mathrm{CH}_{2}$ twisting and $\mathrm{CH}_{2} / \mathrm{CH}_{3}$ bending vibrations (the complete spectral assignment is presented in previous sections). A careful inspection of spectra shows the significant changes in peak frequency and intensities (see the arrow in Figure 4.33a). In particular, the peak around $1090 \mathrm{~cm}^{-1}$ assigned for gauche conformations shifts to higher wave numbers with increase of alkyl chain length (shown in Figure $4.32 \mathrm{~b}$ with scale on right side). The influence of alkyl chain length was also observed in the separation of peak frequencies of symmetric $\left(1123 \mathrm{~cm}^{-1}\right)$ and asymmetric $\left(1140 \mathrm{~cm}^{-1}\right) \mathrm{C}-\mathrm{C}$ stretching vibration as shown in the Figure 4.33b. 


\subsubsection{In micellar solutions}

Raman spectra of micellear solutions for $\mathrm{C} 12 \mathrm{TO}$ C18 are presented in Figure 4.34. The spectral regime corresponds to $\mathrm{C}-\mathrm{C}$ stretching, $\mathrm{CH}_{2}$ twisting and $\mathrm{CH}_{2} / \mathrm{CH}_{3}$ bending vibrations. $100 \mathrm{mM}$ surfactant solution is used and the Raman spectra were measured at $25{ }^{\circ} \mathrm{C}$ except OTAB which was measured at $40{ }^{\circ} \mathrm{C}$. Major spectral differences are found in the $\mathrm{CH}_{2} / \mathrm{CH}_{3}$ bending vibration (near 1450 $\mathrm{cm}^{-1}$ ). Evolution/splitting of the band appear from DTAB to OTAB, which is characteristic for the ordering of the molecules. The $\mathrm{CH}_{2}$ twisting mode at 1300 $\mathrm{cm}^{-1}$ from DTAB to OTAB remains unchanged in frequency, in accordance with finding of Brown [Brown_1987] for the n-alkanes. He noticed that the $\mathrm{CH}_{2}$ twisting mode is relatively insensitive to the extent of hydrocarbon chain order in a given liquid or solid phase.

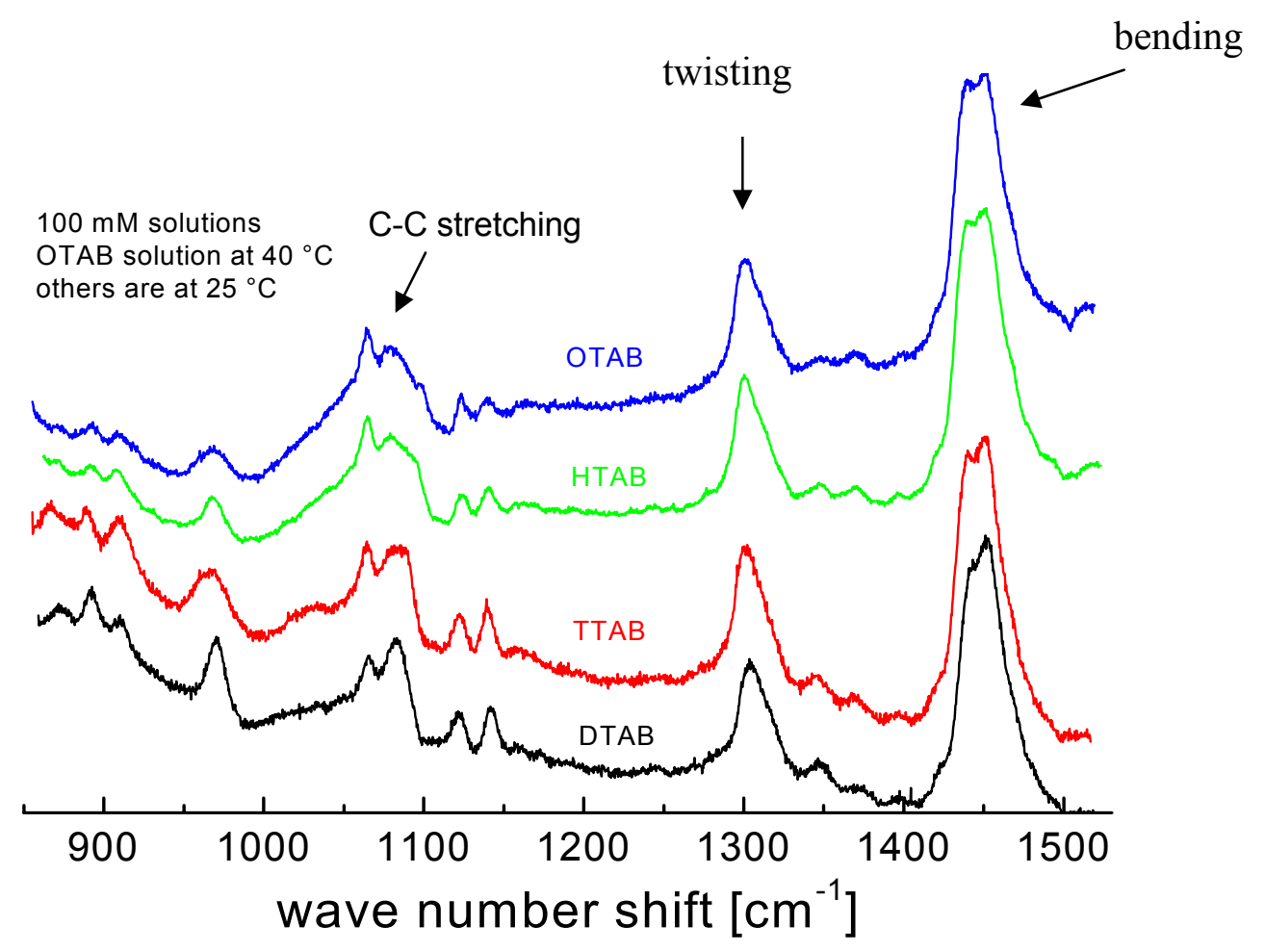

Figure 4. 34: Comparison of Raman spectra in micellar phase, OTAB is measured at $40{ }^{\circ} \mathrm{C}$ and all other surfactant are at $25^{\circ} \mathrm{C}$. (Note that the individual spectra are shifted vertically for clarity.) 


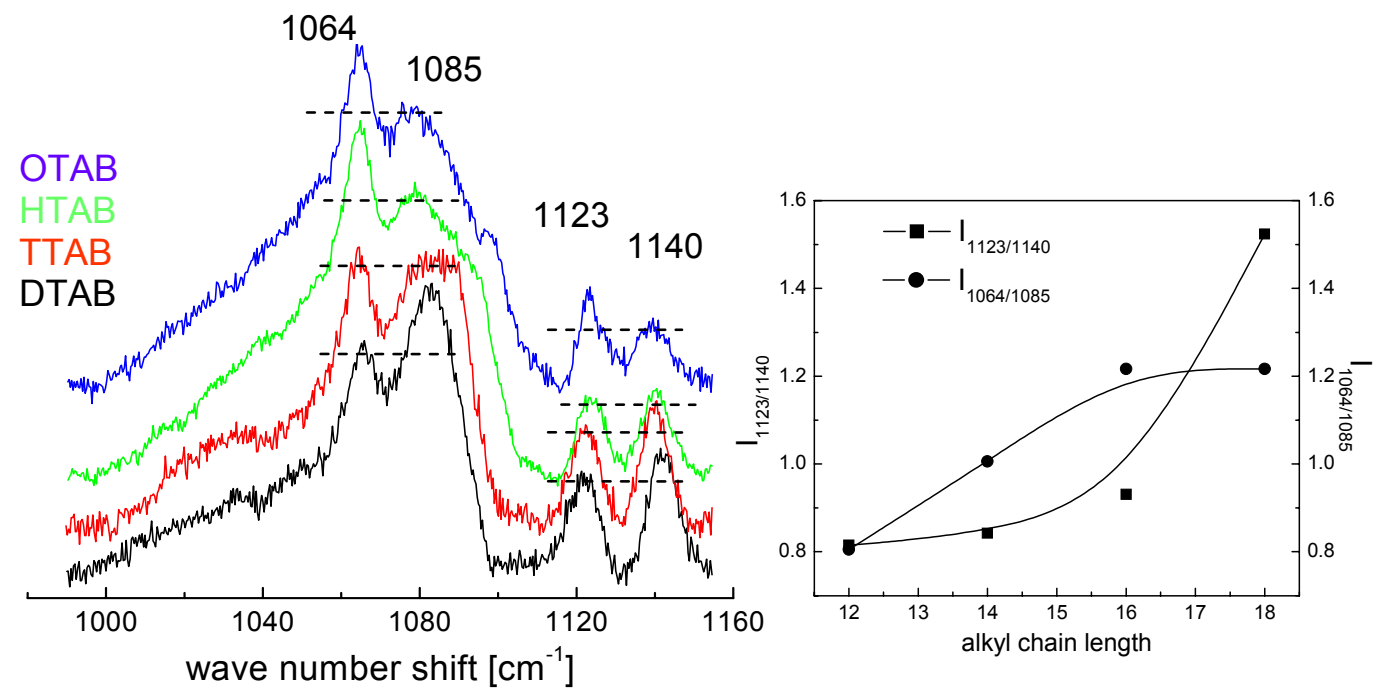

Figure 4. 35: (a) Comparison of Raman spectra in micellar phase showing only in $\mathrm{C}-\mathrm{C}$ stretching regime, OTAB is measured at $40{ }^{\circ} \mathrm{C}$ and all other surfactant are at 25 ${ }^{\circ} \mathrm{C}$. (Note that the individual spectra are shifted in vertically for clarity), (b) peak height ratio of tans to gauche bands

Changes in the relative trans and gauche conformation is presented separately in Figure 4.35a (horizontal dashed lines are used to indicate corresponding intensity levels). This figure clearly demonstrates the relative change in the chain conformations with alkyl chain length. Presence of higher fraction of gauche conformation is indicated by the peak heights in case of DTAB (C12). The fraction of trans conformation increases with increase of alkyl chain length. Ratio of peak heights of $1064 \mathrm{~cm}^{-1}$ to $1085 \mathrm{~cm}^{-1}$, and $1123 \mathrm{~cm}^{-1}$ to $1140 \mathrm{~cm}^{-1}$ are plotted against alkyl chain length in Figure 4.35b. The difference in behavior of theses two ratio is the result of the band profiles. The gauche band near 1085 $\mathrm{cm}^{-1}$ becomes broader with shoulders. While determining the ratio this behavior underestimates the gauche population. Whereas the ratio of trans $\left(1123 \mathrm{~cm}^{-1}\right)$ to gauche $\left(1140 \mathrm{~cm}^{-1}\right)$ band are not affected by such broadening.

The increase of fraction of trans conformations with increase of alkyl chain length in agreement with the fact that the aggregation number and the length of the micelles increases with increase of alkyl chain length. 
Our SANS results provide useful information about the effect of alkyl chain length on aggregation number and the length of the micelles. Figure 4.36 shows the SANS intensity profiles of $100 \mathrm{mM}$ micellar solutions of DTAB, TTAB, HTAB and OTAB.

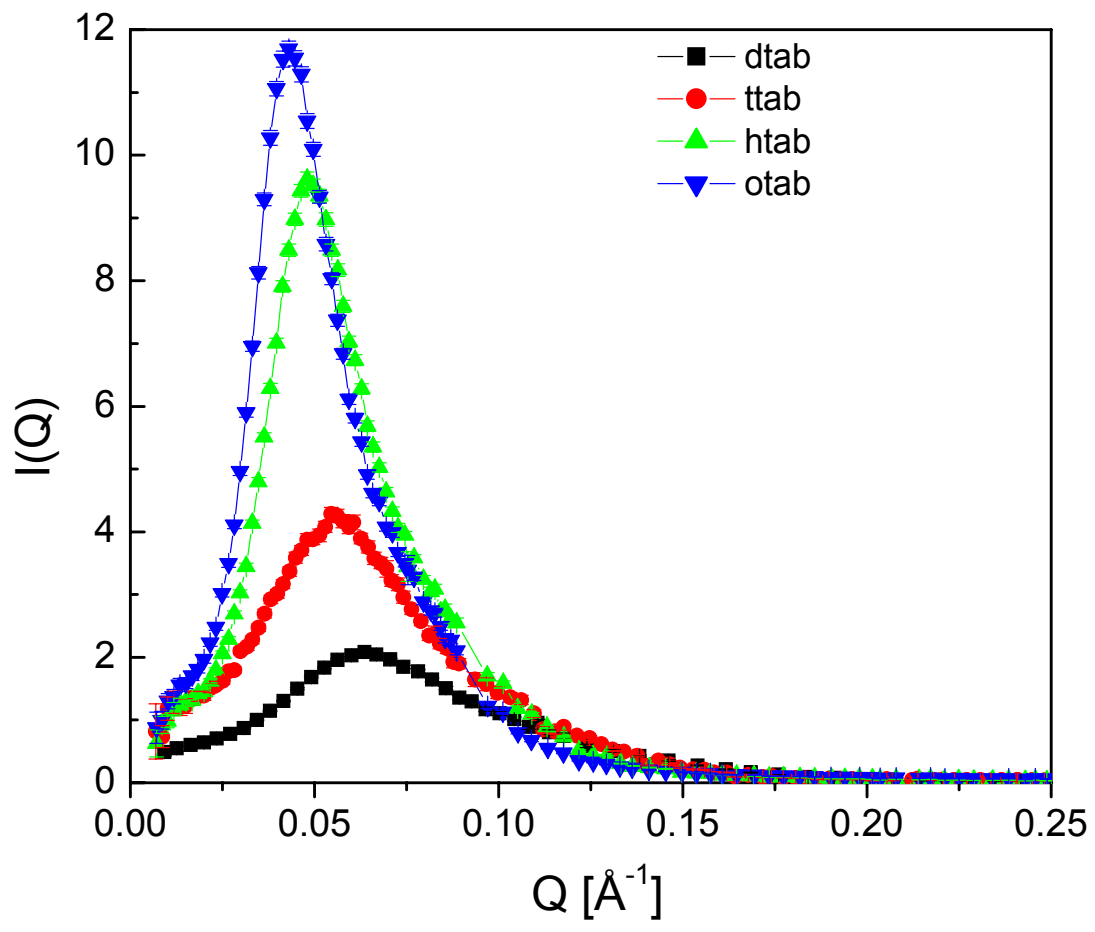

Figure 4. 36: SANS intensity profiles of alkyltrimethylammonium Bromide (C12 to C18) at their $100 \mathrm{mM}$ micellar solution

OTAB was measured at $40^{\circ} \mathrm{C}$, where as the rest were measured at $25^{\circ} \mathrm{C}$ and at ambient pressure. All the four samples show a strong correlation peak indicating the presence of interacting charged micelles in these solutions. As shown in Figure 4.36, the intensity of the correlation peak (the cross section) increases and the peak position shifts to the lower $Q$ values with increase of alkyl chain length. The geometrical parameters such as aggregation number $\left(\mathrm{N}_{\mathrm{agg}}\right)$, degree of dissociation $(\alpha$ ), semi axes are tabulated in Table 4.4 (as obtained by Hayter and Penfold model fitting). It is seen that the aggregation number increases from 71 to 216 with increasing alkyl chain length. This finding in fact agrees well with the degree of dissociation, which decreases with increase of alkyl chain length 
and leads to the larger micelles as indicated by their semimajor axis. The semiminor axes are essentially given by the length of the alkyl chain. These results are consistent with the finding of the Raman measurements, where the fraction of trans conformation increases indicating a higher degree of conformational order with increase of alkyl chain length. The presented SANS data are in good agreement with the finding of Aswal [Aswal_2002]. It is believed that the decrease of aggregation number with decrease of alkyl chain length is due to the lower condensation of counterions on the micelles, which is supported by the fractional charge on the micelles [Aswal_2002].

\begin{tabular}{|c|c|c|c|c|}
\hline Surfactant & Nagg & $\alpha$ & $\begin{array}{c}\text { Semiminor axis } \\
\mathrm{a}=\mathrm{b}(\AA)\end{array}$ & $\begin{array}{c}\text { Semimajor } \\
\text { axis c }(\AA)\end{array}$ \\
\hline DTAB & 73 & 0.29 & 17.4 & 27.0 \\
\hline TTAB & 103 & 0.22 & 20.0 & 34.0 \\
\hline \hline HATB & 171 & 0.14 & 23.8 & 42.2 \\
\hline OTAB & 216 & 0.15 & 26.5 & 46.4 \\
\hline
\end{tabular}

Table 4. 4: Geometrical parameters of micellar solutions of alkyltrimethylammonium bromides at ambient conditions 


\subsubsection{Thermal behavior of Octadecyltrimethylammonium Bromide (OTAB)}

This part focuses on the thermal behavior of aqueous micellar solution of OTAB. In case of TTAB, we have seen that the phase transition from micelles to solid like phase was observed around $5{ }^{\circ} \mathrm{C}$ at ambient pressure. Here, OTAB passes through an intermediate metastable gel phase. With the help of Raman spectra, the following section evidences the formation of metastable gel phase. Figure 4.37 presents the Raman spectra of $100 \mathrm{mM}$ OTAB micellar solution in C-C stretching and $\mathrm{CH}_{2} / \mathrm{CH}_{3}$ bending regime. At room temperature $\left(25^{\circ} \mathrm{C}\right)$, this solution is slightly milky and can be transferred to a clear solution by heating above $40^{\circ} \mathrm{C}$.

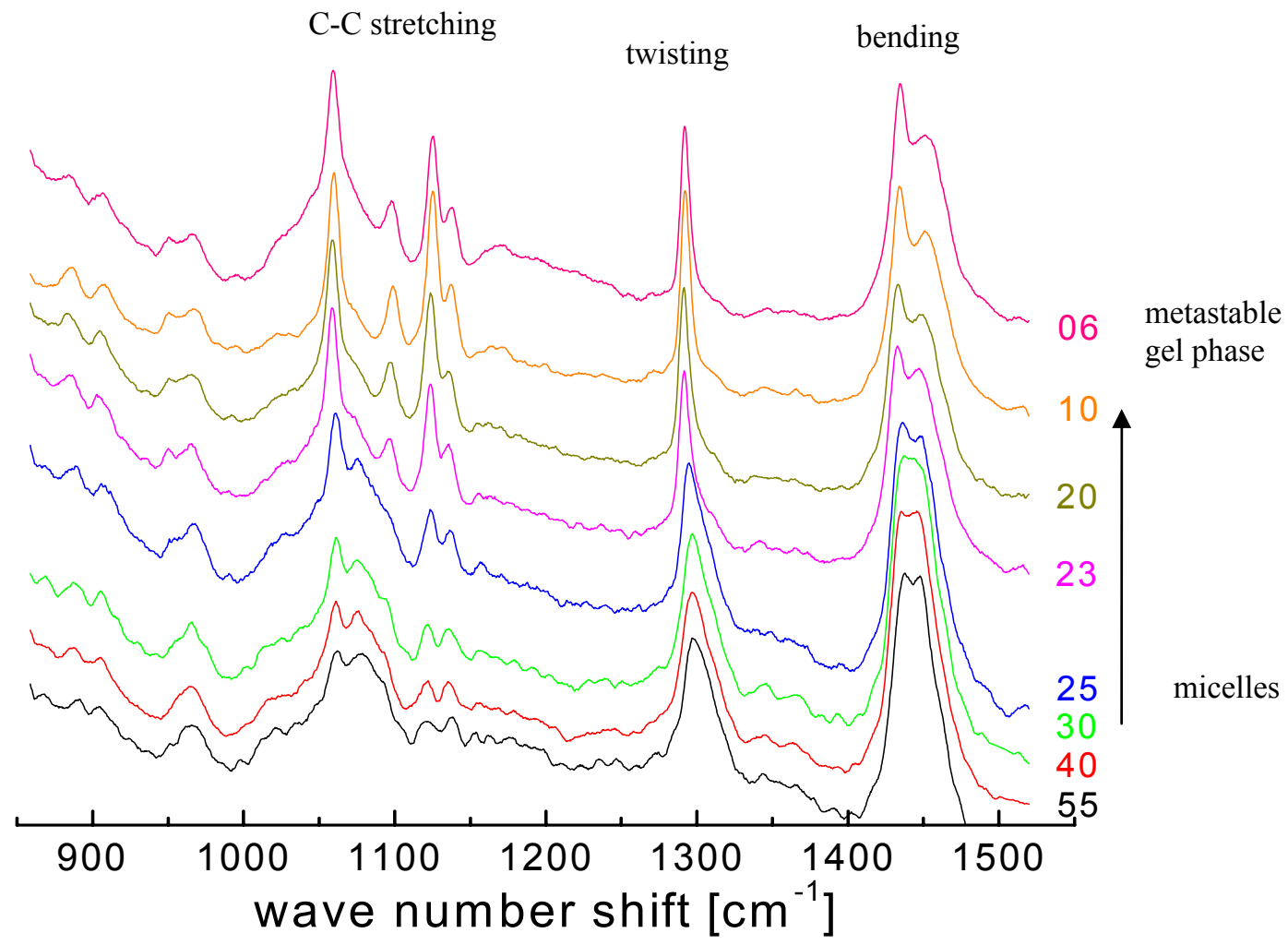

Figure 4. 37: Raman spectra of $100 \mathrm{mM}$ OTAB in C-C stretching, $\mathrm{CH}_{2}$ twisting and $\mathrm{CH}_{2}$ bending regime, the numbers are the temperature in ${ }^{\circ} \mathrm{C}$ at which the spectra are recorded and the arrow indicates direction of the measurements (Individual spectra are shifted for clarity) 
The spectra from $55{ }^{\circ} \mathrm{C}$ to $30{ }^{\circ} \mathrm{C}$ are characteristic of micellar solutions. The changes in the spectral features around $\mathrm{CH}_{2} / \mathrm{CH}_{3}$ bending (near $1450 \mathrm{~cm}^{-1}$ ), twisting (near $1300 \mathrm{~cm}^{-1}$ ), and the $\mathrm{C}-\mathrm{C}$ stretching modes ( from $1000 \mathrm{~cm}^{-1}$ to $1150 \mathrm{~cm}^{-1}$ ) are observed below $25^{\circ} \mathrm{C}$.

In Figure 4.38 Raman spectra are shown for $100 \mathrm{mM}$ OTAB micellar solution in $\mathrm{C}-\mathrm{H}$ stretching regime. The spectra from $70{ }^{\circ} \mathrm{C}$ to $35{ }^{\circ} \mathrm{C}$ are characteristic of micellar solutions. At $25{ }^{\circ} \mathrm{C}$ evolution of the methylene $\mathrm{C}-\mathrm{H}$ asymmetric stretching band appears. The peak intensity reaches that of methylene $\mathrm{C}-\mathrm{H}$ symmetric (near $2848 \mathrm{~cm}^{-1}$ ) stretching band and remains almost at same height.

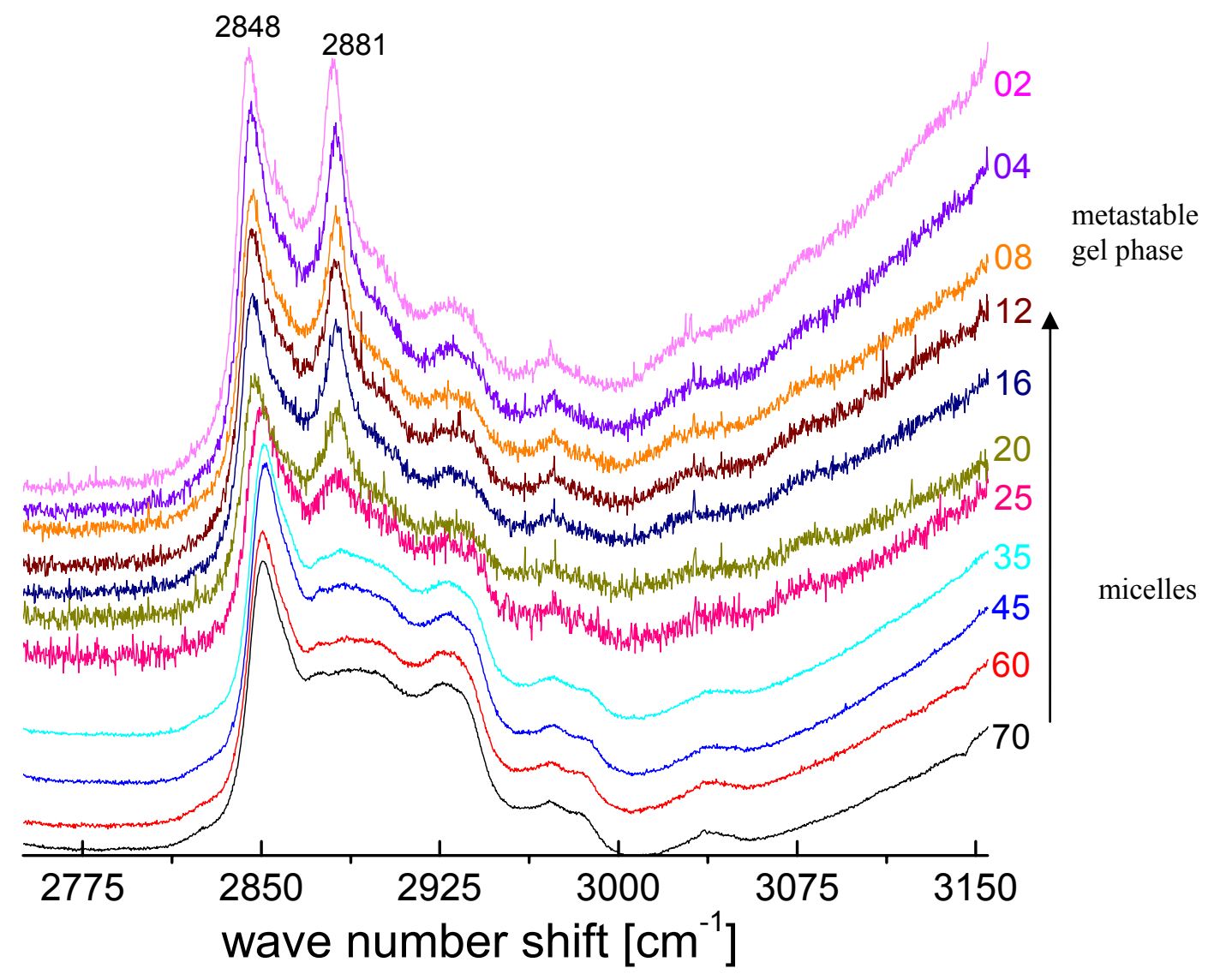

Figure 4. 38: Raman spectra of $100 \mathrm{mM}$ OTAB in $\mathrm{CH}$ stretching regime, the numbers are the temperature in ${ }^{\circ} \mathrm{C}$ at which the spectra are recorded and the arrow indicates direction of the measurements (Individual spectra are shifted for clarity) 
The methylene $\mathrm{C}-\mathrm{H}$ symmetric (near $2850 \mathrm{~cm}^{-1}$ ) and asymmetric (near 2889 $\mathrm{cm}^{-1}$ ) stretching bands decrease to a lower wave number with decreasing temperature. However, these spectral changes (Figures 4.37 and 4.38) are not in good agreement with the spectral features of powder OTAB to say that, the phase transition is from micelles to solid phase and this phase is referred as metastable gel phase. On heating, the back transformation from metastable gel phase to micellar phase is observed around $37^{\circ} \mathrm{C}$ with a hysteresis of about 14 ${ }^{\circ} \mathrm{C}$.

To have an idea, how the Raman spectrum from OTAB solid phase and the spectral changes from solid to micelle phase look like, $100 \mathrm{mM}$ aqueous micellar solution of OTAB was incubated at $-14{ }^{\circ} \mathrm{C}$ for about 24 hours for solidification and then measurements are carried out with increasing temperature. Figures 4.39 and 4.40 present Raman spectrum both in $\mathrm{C}-\mathrm{C}$ skeletal and $\mathrm{C}-\mathrm{H}$ stretching regimes.

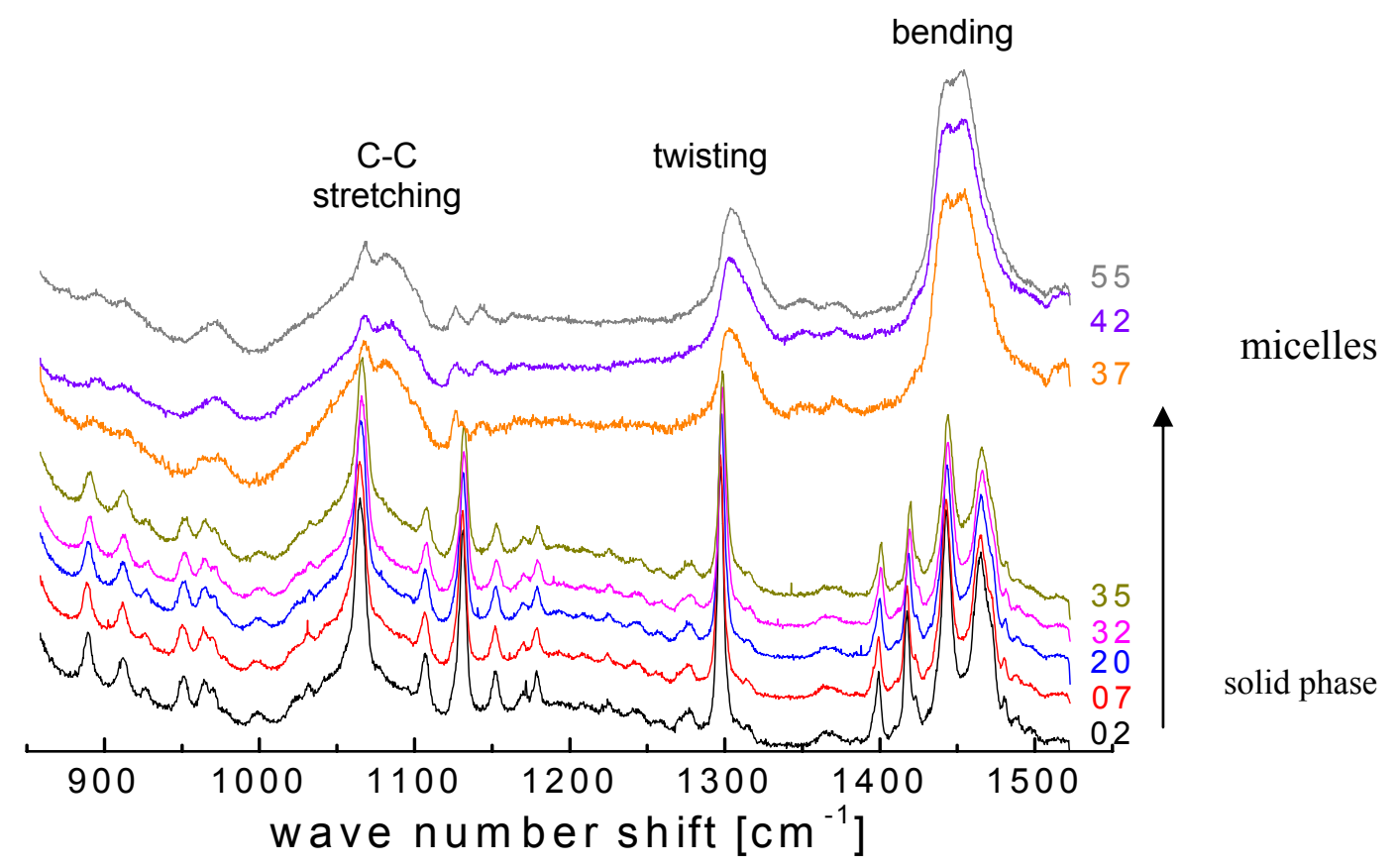

Figure 4. 39: Raman spectra of $100 \mathrm{mM}$ OTAB in C-C stretching, $\mathrm{CH}_{2} / \mathrm{CH}_{3}$ bending regime, the numbers are the temperature in ${ }^{\circ} \mathrm{C}$ at which the spectra are recorded and the arrow indicates direction of the measurements (Individual spectra are shifted for clarity) 
Figure 4.39 presents Raman spectra in C-C stretching and $\mathrm{CH}_{2}$ twisting, $\mathrm{CH}_{2} / \mathrm{CH}_{3}$ bending regime. Spectrum at $2{ }^{\circ} \mathrm{C}$ with sharp bands resembles that of powder OTAB. Significant spectral changes were observed at $37{ }^{\circ} \mathrm{C}$ with increase of temperature. Spectra above $37^{\circ} \mathrm{C}$ temperature resemble that of micellar solutions.

In Figure 4.40, which corresponds to $\mathrm{C}-\mathrm{H}$ stretching regime, the spectrum at $2{ }^{\circ} \mathrm{C}$ resembles that of powder OTAB (not shown) with sharp methylene $\mathrm{C}-\mathrm{H}$ asymmetric (near $2881 \mathrm{~cm}^{-1}$ ) band and the intensity of this band is reasonably higher than that of methylene $\mathrm{C}-\mathrm{H}$ symmetric stretching $\left(2848 \mathrm{~cm}^{-1}\right)$ band. The phase transition from this solid to micelle phase was observed around $37{ }^{\circ} \mathrm{C}$. Frequency of methylene $\mathrm{C}-\mathrm{H}$ symmetric and asymmetric stretching bands increases when entering to the micellar phase. Frequency and intensity of $\mathrm{C}-\mathrm{H}$ asymmetric stretching band overlaps with the broad Fermi resonance band, near $2890 \mathrm{~cm}^{-1}$.

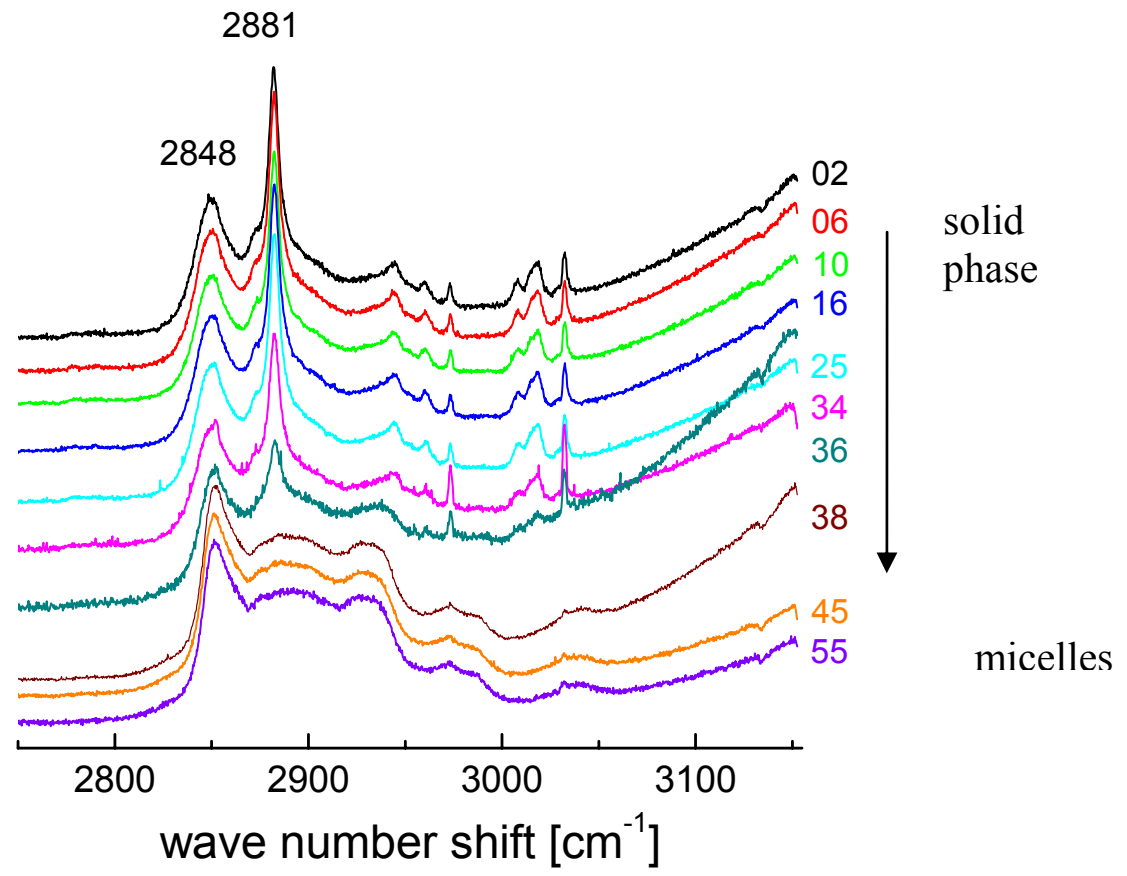

Figure 4. 40: Raman spectra of $100 \mathrm{mM} \mathrm{OTAB}$ in $\mathrm{C}-\mathrm{H}$ stretching regime, the numbers are the temperature in ${ }^{\circ} \mathrm{C}$ at which the spectra are recorded and the arrow indicates direction of the measurements (Individual spectra are shifted for clarity) 
It is worth to note that, phase transition from metastable gel phase to micellar phase occurs also around $37^{\circ} \mathrm{C}$ as well.

The measurements from metastable gel to micellar phase and from solid to micellar phase are compared. The striking differences were observed in peak height ratio trans to gauche conformations, and band profile of $\mathrm{CH}_{2} / \mathrm{CH}_{3}$ bending region (around $1450 \mathrm{~cm}^{-1}$ ) and peak height ratio of asymmetric to symmetric methylene $\mathrm{C}-\mathrm{H}$ stretching vibrations. Figure 4.41 presents the peak height ratio of trans $\left(1126 \mathrm{~cm}^{-1}\right)$ to gauche $\left(1085 \mathrm{~cm}^{-1}\right)$ bands. This plot demonstrates that in metastable gel phase too the hydrophobic part of the surfactant molecules is in extended chain form with fraction of gauche conformation when compared with that of solid phase.

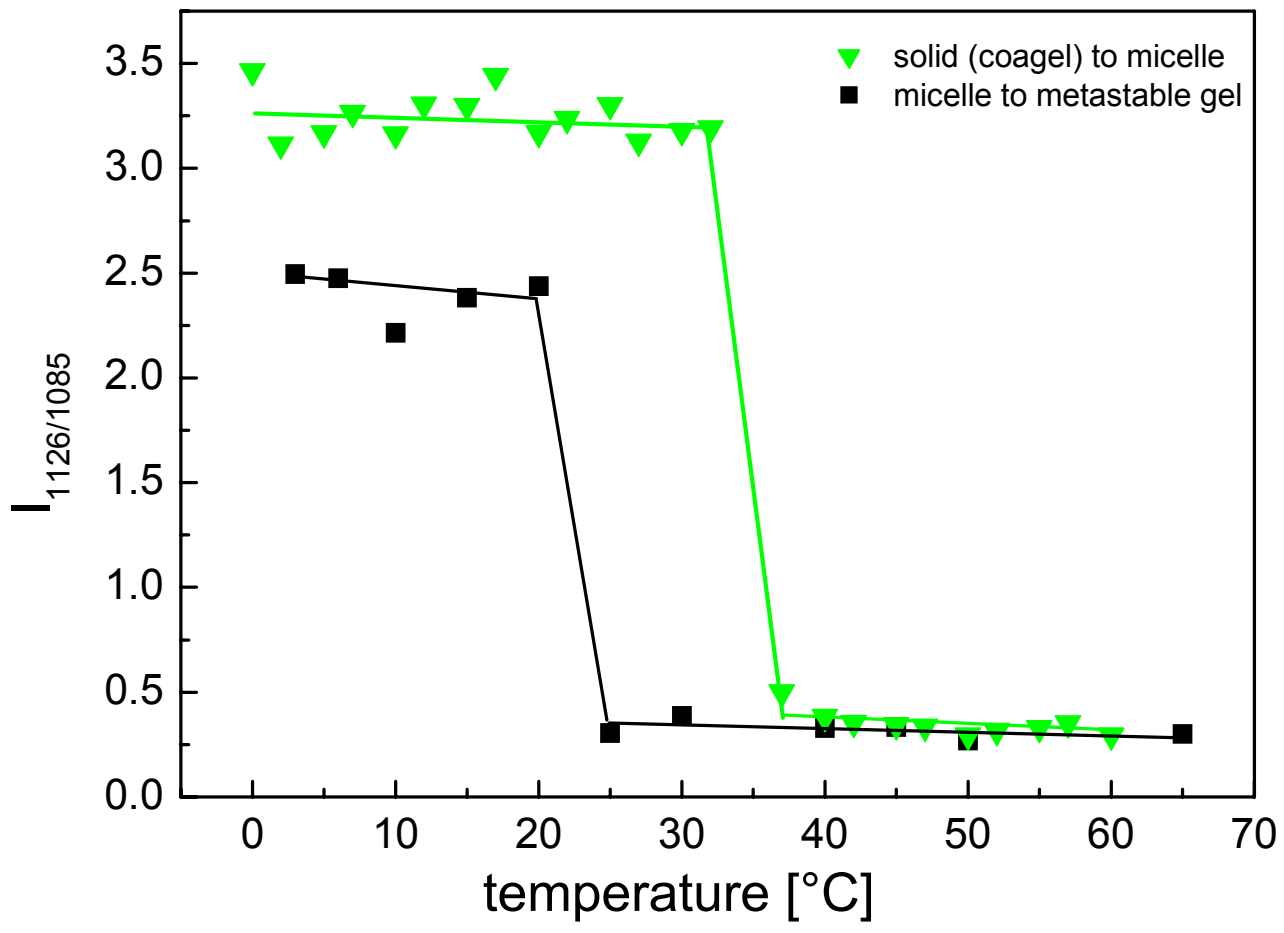

Figure 4. 41: Ratio of peak height of trans $\left(1126 \mathrm{~cm}^{-1}\right)$ to gauche $\left(1085 \mathrm{~cm}^{-1}\right)$. Data are from phase transition of micelles to metastable gel phase and from solid (coagel) to micelles. Solid lines are just an eye guide 
The other important spectral regime which showed a significant spectral difference between the metastable gel phase and the solid phase was $\mathrm{CH}_{2} / \mathrm{CH}_{3}$ bending mode. As shown in Figures 4.37 and 4.39, in case of solid phase, the spectrum exhibit sharp peak, whereas in metastable gel is not (see Figure 4.42). Figure 4.42 shows the photographs of micellar solution, metastable gel phase and solid phase in comparison with the Raman spectrum in $\mathrm{CH}_{2} / \mathrm{CH}_{3}$ regime. Photographs of micellar solution was taken when it was above $40{ }^{\circ} \mathrm{C}$, metastable gel is pictured below $23{ }^{\circ} \mathrm{C}$ and the solid phase is pictured around $10{ }^{\circ} \mathrm{C}$, all these samples were in Hellma double walled quartz cuvette, these cuvettes were hold tighten using rubber band (red colored). Again to mention here, solid phase was achieved by incubation of OTAB micellar solution at $-14{ }^{\circ} \mathrm{C}$.

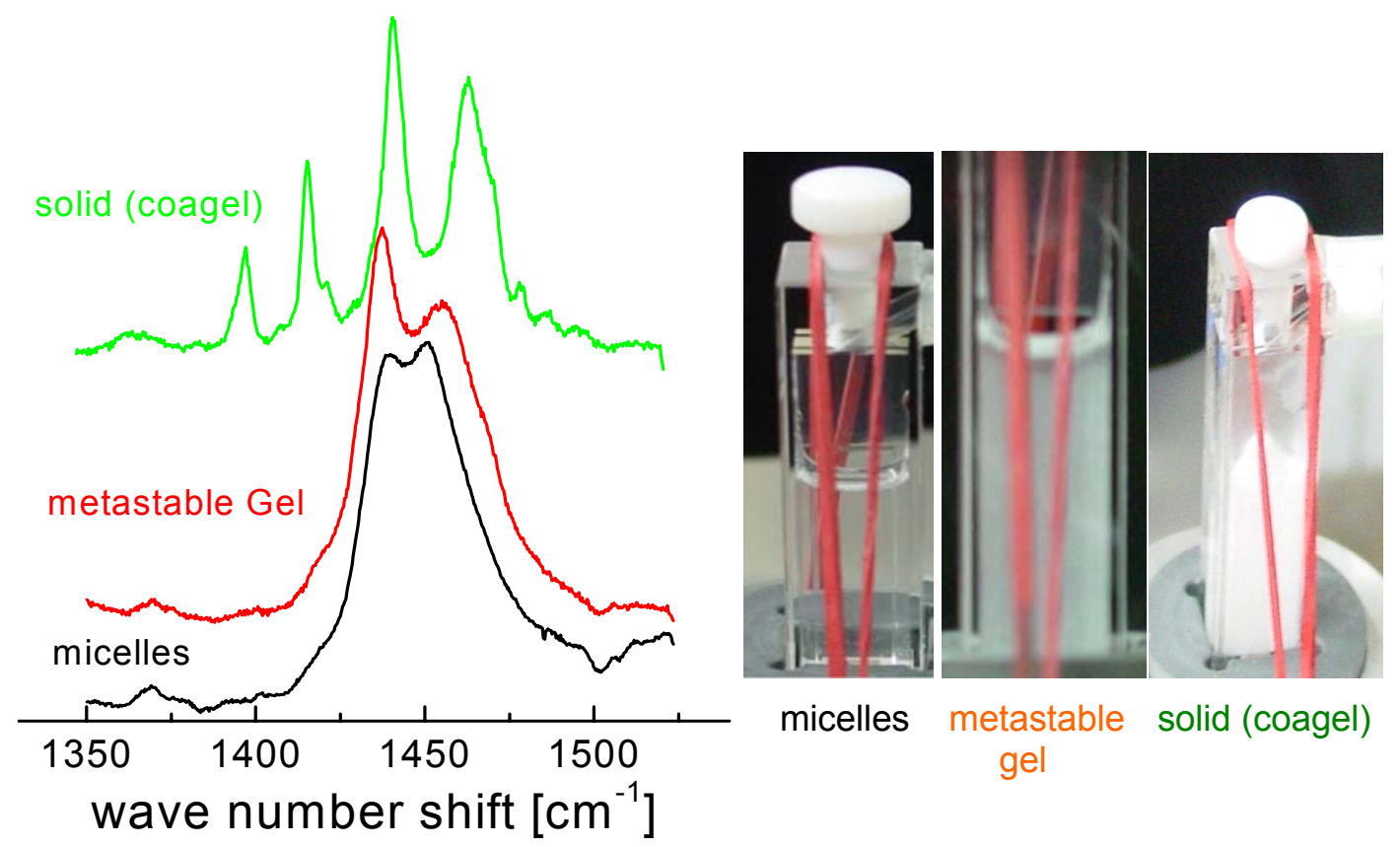

Figure 4. 42: Raman spectra at three different phase (micelles, metastable gel and solid (coagel)); in comparison with the photographs of the same (see the text for details). Red bands in the photographs are of rubber band used for tightening the cuvette 
Figure 4.43 presents peak height ratio of asymmetric $\left(2889 \mathrm{~cm}^{-1}\right)$ to symmetric $\left(2850 \mathrm{~cm}^{-1}\right)$ methylene $\mathrm{C}-\mathrm{H}$ stretching against temperature. With decrease of temperature OTAB is transferred to metastable gel phase near $23{ }^{\circ} \mathrm{C}$ (black square symbols in Figure 4.43). Measurements with increase of temperature for the same metastable gel phase end up with transition to micelles around $38^{\circ} \mathrm{C}$ (red circle) showing a hysteresis of about $14{ }^{\circ} \mathrm{C}$. Solid to micelle phase transition occur also at $38{ }^{\circ} \mathrm{C}$. Below $35^{\circ} \mathrm{C}$ the plot shows a clear difference in metastable gel phase and solid (coagel) phase. Since, the solid phase has more of well ordered phase and hence, the magnitude of ratio of peak height is high in comparison with the metastable gel phase. After the phase transition from both, metastable gel phase and solid phase the ratio is almost same, since it's in the micellar phase.

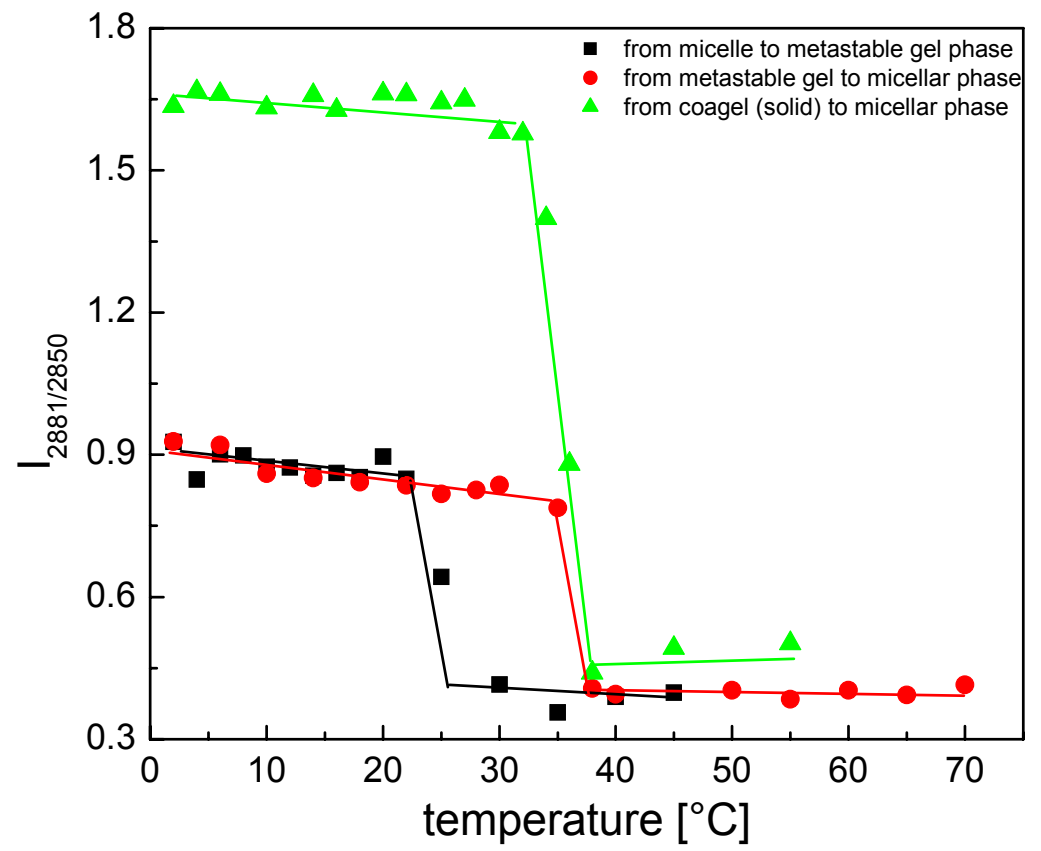

Figure 4. 43: Ratio of peak height of methylene asymmetric and symmetric $\mathrm{C}-\mathrm{H}$ stretching band. Data are from phase transition of metastable gel phase to micelles and vise versa and from solid (coagel) to micelles. Solid lines are just an eye guide 
In case of TTAB, it has been already discussed that increase in ratio of trans to gauche conformations is an indication of phase transition from micelles to solid phase. It has been shown that micellar phase exhibits both, trans and gauche conformations, after the phase transition (both at ambient pressure and applied pressure) the trans conformations are dominated over gauche, indicating the presence extended alkyl chains. OTAB too attains the same explanation for ratio of trans and gauche showing difference in metastable gel phase and solid phase. Solid phase showing extended chain configuration, whereas the metastable gel phase with fraction of gauche conformations. These results are consistent when compared with literature for the systems of phospholipid dispersions (membranes) under pressure or at low temperatures [Wong_1983c, Wong_1984, Wong_1985, Wong_1985a, Wong_1985b, Cameron_1980, Huang_1982, Yellin_1977, Gaber_1977, Lippert_1971, Wu_1982, and Casal_1984]. Wu et al. has found the differences in the peak height ratio of $2936 \mathrm{~cm}^{-1}$ to $2883 \mathrm{~cm}^{-1}$ for the systems of 1-Stearoyllysophosphatidylcholine (1-Stearoyl PC) and distearoylphosphatidylcholine (Distearoyl PC) dispersions. The lipid matrix forming the lamellar structure for 1-Stearoyl PC molecule is more ordered than the gel form of bilayer organization of Distearoyl PC molecules. P T T Wong [Wong_1985b] has showed that, the ratio of $2930 \mathrm{~cm}^{-1}$ to $2880 \mathrm{~cm}^{-1}$ for the system of Dipalmitoylphosphatidylcholine (DPPC) in non-incubated and incubated (at ${ }^{\circ} \mathrm{C}$ for 3 weeks) state. The ratio suggests that, below $20{ }^{\circ} \mathrm{C}$, the non-incubated sample of DPPC has higher gauche conformations in comparison with the incubated sample of DPPC. Cameron [Cameron_1980] reports infrared spectra of DPPC, in $\mathrm{CH}_{2} / \mathrm{CH}_{3}$ bending regime a peak near $1468 \mathrm{~cm}^{-1}$ was observed which is a characteristic of gel phase with all trans chains packed in a hexagonal subcell lattice with no intermolecular coupling since the rotational motion of the alkyl chain is active in the gel phase. Reducing temperature (below $-40{ }^{\circ} \mathrm{C}$ ) results in crystal field splitting characteristic for monoclinic subcell packing. Kaneshina et al. [Kaneshina_1990] have presented the differential scanning calorimetric (DSC) study of the thermotropic phase behaviors in alkylterimethylammonium Bromide (C14 to C18) surfactants. They pointed out 
that $\mathrm{HTAB}$ and OTAB shows a metastable gel phase on cooling the micellar solutions. The solid phase was observed by storing the micellar solution at $5{ }^{\circ} \mathrm{C}$ for 1-6 days. On heating, DSC curves show strong endothermic peaks near 28 ${ }^{\circ} \mathrm{C}$ and $37^{\circ} \mathrm{C}$. They assign these peaks to the phase transition temperature from metastable gel phase to supercooled micelles and from solid phase to micelles respectively. However, present Raman scattering measurements showed no difference in the phase transition temperatures $\left(37^{\circ} \mathrm{C}\right)$ from both, solid to micelles and metastable gel to micelles (see Figure 4.42). Appearance of metastable gel phase in case of octadecyltrimthylammonium Chloride (OTAC) has been reported by Kodama et al. [Kodama_1986]. With the help of DSC results, he pointed out that, transferring of gel phase into a coagel phase can be done with suitable annealing treatment. The coagel phase thus obtained was transferred in to the gel phase; the DSC thermogram indicated the existence of stable gel phase in specified temperature region. The importance of annealing has been explained as the gel phase is basically bilayers of the OTAC molecule with loosely bound water molecules in between them and this water will be released outside the bilayers by annealing treatment and exists as bulk free water in coexistence with the resulting coagel. Tsuchiya et al [Tsuchiya_1994) describes the structure of coagel and gel phase with a model (shown in Figure 2.4) showing that the gel phase of surfactants is a bilayer with sufficiently large amount of water (thickness of about $1000 \AA$ ) in between, whereas coagel phase is bilayer too, but with very tiny amount of water (thickness of about $10 \AA$ ).

The Raman spectrum of OTAB (see Figures 4.38 and 4.40 in frequency regime of $3150 \mathrm{~cm}^{-1}$, where the rise in intensity towards the water peak which appear near $3450 \mathrm{~cm}^{-1}$ ), shows the presence of water both in solid and metastable gel phase. Similar observation was found also for TTAB where the water was within the solid phase and later squeezes out (see appendix 4 for details). By visual inspection of cuvettes it was noticed that, the water molecules were squeezed out of the coagel and remain in coexistence with the solid. The thickness of the bilayer can be determined with X-ray diffraction method. However, the 
metastable gel phase was indicated by the appearance of Bragg diffraction-like peaks in SANS measurements. Figure 4.44 presents the SANS intensity profiles of $100 \mathrm{mM}$ OTAB at different temperatures.

Bottom part of the Figure 4.44 corresponds for the intensity profile for $100 \mathrm{mM}$ OTAB micellar phase at $40^{\circ} \mathrm{C}$, which shows a correlation peak for presence of micelles. OTAB forms metastable gel phase below $25{ }^{\circ} \mathrm{C}$, the SANS intensity profiles (at 25, 15 and $10^{\circ} \mathrm{C}$ ) in the upper part of the Figure 4.44 shows diffraction like peaks with uniform spacing. Thickness of the bilayers of OTAB in metastable gel phase was estimated (using the Bragg diffraction-like peak at lowest $Q$ corresponds to the long spacing of the metastable gel phase) to be around $600 \AA$. In the case of solid phase, X-ray diffraction measurements were done to determine the bilayer thickness and it was about $29 \AA$ (details are given in appendix 3 ), which is almost the length of the surfactant molecule.

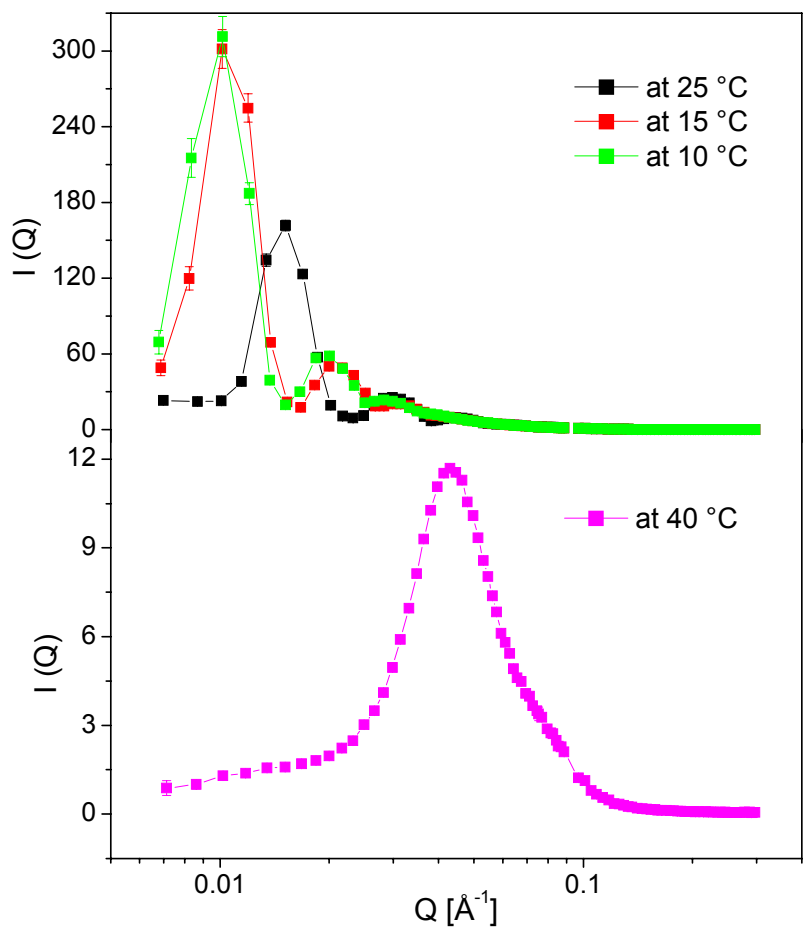

Figure 4. 44: SANS intensity profiles of $100 \mathrm{mM}$ OTAB, upper part is below $25{ }^{\circ} \mathrm{C}$ (gel phase), bottom part corresponds for micellar phase. Y-axis is different for both parts 


\subsubsection{Influence of pressure on HTAB}

Attempts to understand the influence of pressure on phase transition was done, the phase transition is mostly affected by the kinetics. With applied measurements are carried out for $50 \mathrm{mM} \mathrm{OTAB}$ at $52{ }^{\circ} \mathrm{C}$, but there was no evolution of sharp bands in the Raman spectra (not shown), which are characteristic of metastable gel phase or solid phase. The spectral features near 1300 bar vanish leaving behind only the background. Later, while cleaning the pressure cell it was found that the solid/crystallites were settled at the bottom (well below the sample window) and, hence there were no sharp peaks in the spectrum corresponding to the solid phase.

Similar observation was found also for the HTAB, with step by step increase of pressure leads to the settling down of crystallites at the bottom of the sample window, and results in the unstructured spectra. However, the phase transition from micellar solution to solid phase probably through a metastable gel phase was observed for $50 \mathrm{mM}$ OTAB with kinetics measurement at 800 bar pressure. Figure 4.45 presents Raman spectrums measured at 800 bar along with one spectrum measured at 100 bar.

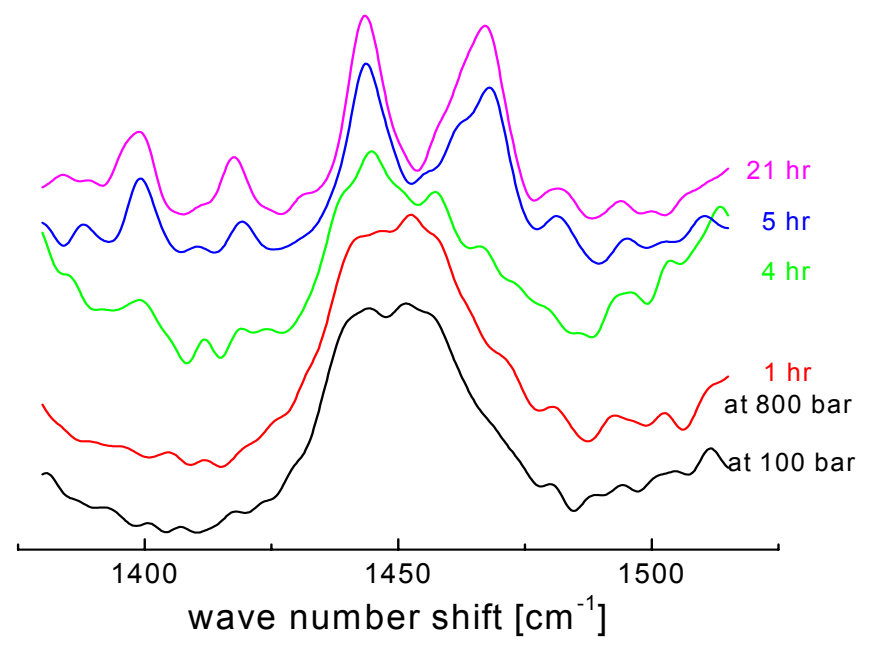

Figure 4. 45: $50 \mathrm{mM}$ aqueous micellar solution of HTAB, spectrums measured at 800 bar as a function of time, spectrum with black color is measured at 100 bar. All measurements are at $25^{\circ} \mathrm{C}$ (spectra are shifted vertically for clarity) 
The spectrum at 100 bar and first spectrum at 800 bar are quite similar showing that they both are in micellar phase. In time scale of hours the spectral features change and a stage is reached, where characteristic of solid phase is seen. However, the formation of metastable gel phase can not be ruled out. The fourth spectra (4 hour) at 800 bar is in between micellar and solid/coagel phase, expected to be the metastable gel phase.

By the visual inspection at ambient pressure, it is observed that, on cooling the formation of metastable gel phase was not reproducible. However, a reproducible metastable gel phase can be observed by incubating at $5{ }^{\circ} \mathrm{C}$. First micellar solutions turns to the metastable gel phase with single (homogenous) phase and then to solid phase in coexistence with water which is squeezed out from the metastable gel phase. Figure 4.46 demonstrates the kinetics of phase transition of $100 \mathrm{mM} \mathrm{HTAB}$ at $5{ }^{\circ} \mathrm{C}$ and at ambient pressure. Cuvette 1 , shows that the maximum portion is metastable gel phase (see the black arrow) and little part (see the blue arrow) is in the solid phase (note that, 30 minutes before the commencement of formations of metastable gel phase the solution was clear at 5 ${ }^{\circ} \mathrm{C}$ and then transferred to a homogeneous metastable gel phase, after few hours (5-6 hours) the solidification started). Cuvette 2, shows the continued solidification, it took around 15 hours to reach this stage from Cuvette 1, complete solidification occurred after another 9 hours from cuvette 2 as shown in cuvette 3. Cuvette 3 , shows also the coexistence of solid phase with liquid (green arrow). At room temperature, the Raman spectrum (not shown) was measured only for the clear liquid part, the measurement has spectral features characteristic of micellar solutions.

The formation of metastable gel phase in HATB is observed also by Kaneshina et al. [Kaneshina_1990] during the differential scanning calorimetric (DSC) measurements. The coexistence of solid phase with liquid solution is also shown by Debye and Anacker [Debye_1951]. 


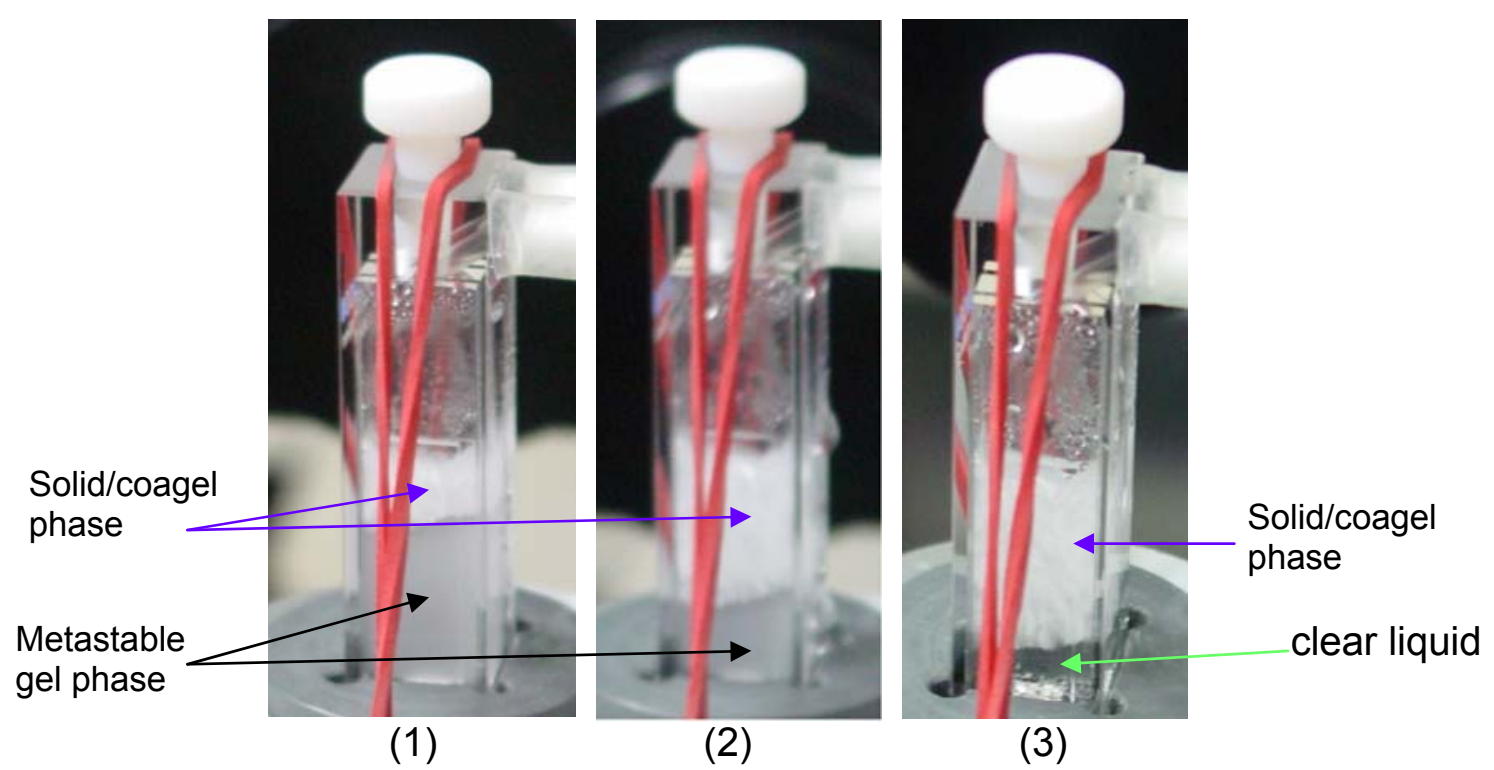

Figure 4. 46: Representative pictures of kinetics of phase transition of $100 \mathrm{mM} \mathrm{HTAB}$ at $05^{\circ} \mathrm{C}$ (red colored rubber band is as a support for the cuvette)

\section{Schematic of phases of alkyltrimethylammoinum brobides}

From the present all experimental informations, the probable phases of alkyltrimethylammonium bromides is shown in Figure 4.47.

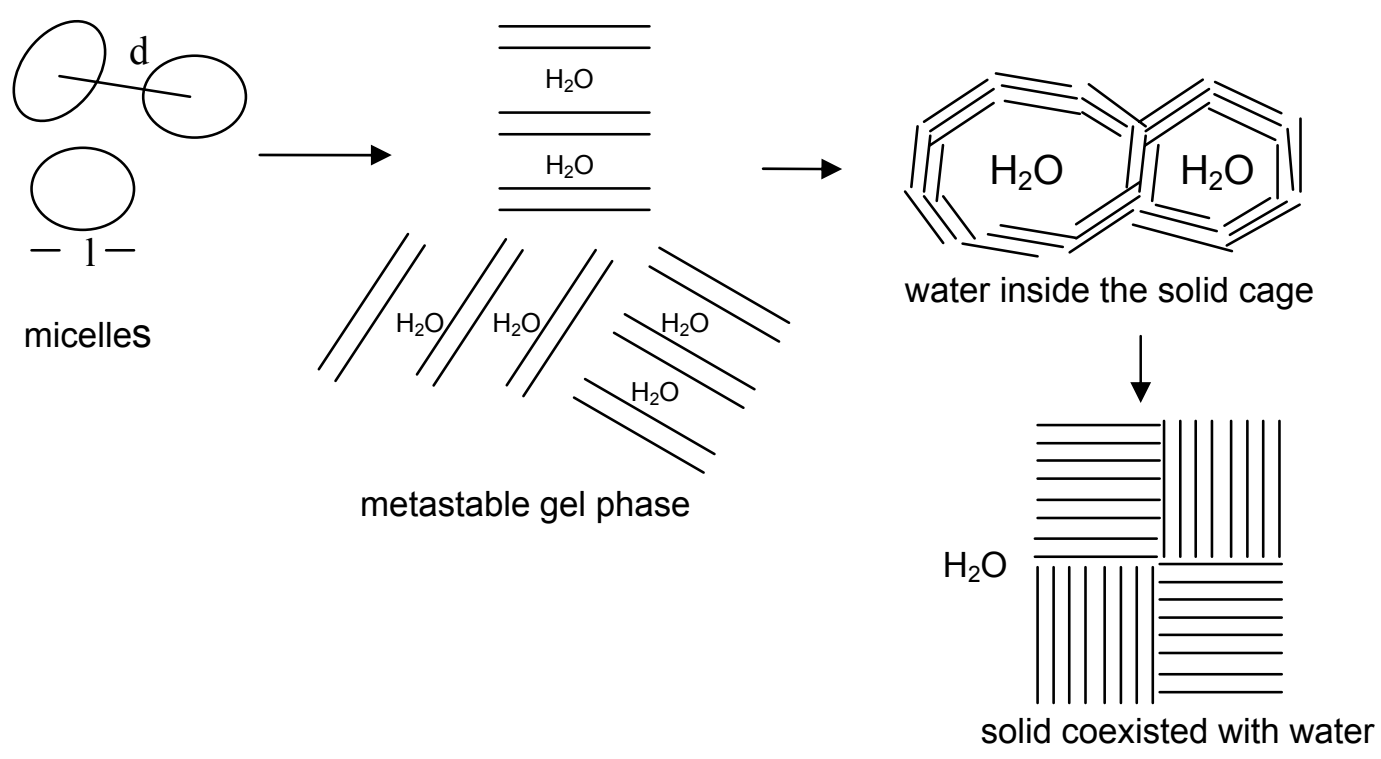

Figure 4. 47: Schematic of the illustration of different phases of alkyltrimethylammoinum bromides 
This scheme shows the micelles of length 1 and the distance $d$ between them. Metastable gel phase is the assembly of bilayers of surfactants with large amount (around $600 \AA$ for OTAB) of water in between them. The presence of water inside the cage of the solid phase is shown, which later squeezes out and coexisting with the soild. The obtained Raman and SANS experimental data supports the scheme shown above.

At ambient conditions the surfactants are in micellar phase. With application of temperature/pressure TTAB shows a clear phase transition from micelles to solid phase. At the beginning the water was inside the cage of the solid phase and later squeezes out and coexists with liquid (see appendix 4).

Higher alkyl chain length surfactants (C16 and C18) showed a metastable gel phase with application of temperature/pressure. The solid phase was achieved by the incubation of the micellar solution at around $-14{ }^{\circ} \mathrm{C}$ for a period of 24 hours. For OTAB, the Figures 4.38 and 4.40 evidences the presence of water in both metastable gel and solid phase (by the raising peak near $3100 \mathrm{~cm}^{-1}$ which is starting point of the water peak around $3450 \mathrm{~cm}^{-1}$ ).

\subsection{Conclusion}

The second part of the Chapter 4 discussed the influence of electrolyte, $\mathrm{NaBr}$ on aqueous micellar solution of TTAB at ambient conditions and then on the phase transition temperature or pressure, this is followed by the influence alkyl chain length in both, dry powder samples and micellar solutions.

At ambient conditions the micellar length and hence the aggregation number increases with addition of electrolyte, $\mathrm{NaBr}$. This is shown by the reduction in the intensity of the correlation peak (structure factor) in SANS intensity profiles and also by the increase of fraction of trans conformations in Raman spectra by the addition of electrolyte. The increased length of the micelles has a pronounced effect on the phase transition temperature or pressure. There is a clear shift of the solidification/crystallization towards lower pressures and higher temperatures. 
That allows one to estimate the dependence of activity coefficient in the liquid solution from the salt concentration.

Raman spectra of DTAB, TTAB, HTAB and OTAB dry powders show a spectral difference with increase of alkyl chain length, especially near the longitudinal accordion mode (LAM). The frequency of LAM is inversely proportional to the alkyl chain length. In case of micellar solution the aggregation number increases and hence the fraction of trans conformation increases with increase of alkyl chain length with the slight ordered micellar core.

The surfactants with alkyl chain length above $14 \mathrm{CH}_{2}$ group shows well pronounced intermediate metastable gel phase. The formation solid phase in case of OTAB can be observed by incubating the micellar solution around $-14{ }^{\circ} \mathrm{C}$. Evidences for the formation of metastable gel phase are well indicated by the Raman spectra. SANS provided the bilayer thickness of the metastable gel phase and was turned out to be around $600 \AA$. X-ray diffraction measurements of solid phase indicated the bilayer thickness of about $29 \AA$. With the visual inspection it was observed that the metastable gel phase is a uniform/homogeneous and the coagel phase exist in coexistence with liquid water.

Using our Raman and SANS experimental data a schematic of the illustration of different phases of alkyltrimethylammonium bromides is shown in Figure 4.47. 


\section{Summary}

In this work, the combination of Raman spectroscopy and Small Angle Neutron scattering (SANS) have been used for the first time, to understand the aggregational behavior of micelles in micellar solutions under different external conditions like temperature, pressure, surfactant concentration and the electrolyte concentration. This comprises the aggregation to solid phase and the growth of micelles due to electrolytes.

The dynamics of the micellar assemblies were analyzed with the help of Raman spectroscopy. The characteristic vibrational bands, their frequency positions, widths, peak height ratios of selected bands are found to be very sensitive to external parameters like temperature, pressure and electrolyte concentration. At ambient conditions, the Raman spectra resembles the spectra of hydrocarbons with broad vibrational bands and almost equal amount of trans and gauche conformations indicating the disorder in the micelles. An increase in the trans to gauche ratio and hence the ordering of the surfactants was achieved by decrease in the temperature of the micellar solutions. An increase of pressure has the similar kind of influence on the spectral behavior. The phase transitions observed with the influence of temperature and pressure are indicated in the discontinuities of the peak frequency, peak width and peak height ratios. Neutron scattering provides the structural information of the micelles. The intermicellar distance as well as their shape were calculated directly from the SANS intensity profile. SANS experimental data were fitted with well known Hayter-Penfold model to extract the structural parameters such as aggregation number, charge and size of the micelles.

Aqueous micellar solution of tetradecyltrimethylammonium bromide (TTAB) at ambient conditions shows an increase of aggregation number with increase in surfactant concentration. With the application of temperature and pressure the 
phase transition from micellar solution to solid phase was evidenced in the decrease in the SANS intensity profile. This phase transition was directly proven by the use of Raman spectroscopy, where, the spectra after the phase transition resemble that of the dry powder TTAB surfactant.

At ambient conditions aqueous micellar solutions of various alkyltrimethylammonium bromide surfactants (with alkyl chain length from $\mathrm{C} 12$ to C18) show an increase of micellar length and hence, the aggregation number with increase in alkyl chain length. Thermal behavior of surfactant with alkyl chain length $\mathrm{C} 16$ and $\mathrm{C} 18$ shows an intermediate metastable gel phase on cooling and was clearly demonstrated by Raman spectra and neutron scattering. 


\section{Zusammenfassung}

In dieser Arbeit wurde zum ersten Mal die Kombination von Ramanspektroskopie und Neutronenkleinwinkelstreuung (SANS) benutzt, um das Aggregationsverhalten mizellarer Lösungen unter verschiedenen äußeren Bedingungen wie Temperatur, Druck, Tensid- und Elektrolytkonzentration zu verstehen. Das umfasst die Aggregation zu hydrierten Kristallen ebenso wie das durch Elektrolyte verursachte Wachsen der Mizellen.

Mithilfe der Ramanspektroskopie wurden die dynamischen Eigenschaften von mizellaren Objekten untersucht. Es zeigte sich, dass charakteristische Schwingungsbanden, deren Frequenzen, Halbwertsbreiten sowie geeignet gewählte Peakhöhenverhältnisse auf äußere Parameter wie Temperatur, Druck und Elektrolytkonzentration sehr empfindlich reagieren. Unter Standardbedingungen ähneln die Ramanspektren denen der Kohlenwasserstoffe mit breiten Schwingungsbanden und einem im wesentlichen gleichen Maß an trans- und gauche-Konformationen; darin zeigt sich die Unordnung innerhalb der Mizellen. Durch Abkühlung der mizellaren Lösungen wurde ein Anstieg des trans-zu-gauche-Verhältnisses und somit eine Ordnung der amphiphilen Moleküle erreicht. Druckerhöhung zeigt einen ähnlichen Einfluss auf die spektralen Eigenschaften. Die durch Temperatur oder Druck induzierten Phasenumwandlungen waren durch Unstetigkeiten in der Schwingungsfrequenz, Peakbreite und Peakhöhenverhältnisse gekennzeichnet. Die Neutronenstreuung liefert strukturelle Informationen über die Mizellen. Der intermizellare Abstand wurde direkt aus den SANS-Profilen berechnet. Die experimentellen SANSDaten wurden an das bekannte Hayter-Penfold-Modell angepasst, um strukturelle Parameter wie Aggregationszahl, Ladung und Größen der Mizellen zu ermitteln.

Wässrige Lösungen von Tetradecyltrimethylammoniumbromid (TTAB) zeigen unter Standardbedingungen einen Anstieg der Aggregationszahl mit der Tensid- 
Konzentration. Die temperatur- und druckinduzierten Phasenumwandlungen von der mizellaren Lösung in einen hydrierten Kristall zeigten sich in einer Abnahme der SANS-Intensitäten. Diese Phasenumwandlungen wurden durch die Ramanspektroskopie verifiziert, da die Spektren dann denen des reinen TTABPulver ähneln.

Mizellare Lösungen von verschiedenen Alkyltrimethylammoniumbromid-Tensiden (mit Kettenlängen von 12 bis 18 Kohlenstoffatome) zeigen unter Standardbedingungen mit der Kettenlänge ein Anwachsen der Mizelllänge und somit der Aggregationszahl. Das thermische Verhalten der C16- und C18Tenside ist durch eine intermediäre Gelphase beim Abkühlen gekennzeichnet. Dies wurde eindeutig durch die Ramanspektroskopie und durch die Neutronenstreuung nachgewiesen. 


\section{Appendix}

\section{Appendix 1}

The number of micelles $(n)$ does not change with applied pressure up to 1000 bar (Figure 4.19), the geometrical parameter of the micelles remain almost same till 1000 bar. However, a slight increase in the correlation peak is observed and is attributed to the change in the volume (density) of the solvent $\left(D_{2} O\right)$ at higher pressures (1000 bar). This can be explained quantitatively as follows;

Scattering intensity at $Q=0$ is given by:

$$
\begin{aligned}
& \mathrm{I}(0)=\mathrm{n}\left(\rho-\rho_{\mathrm{s}}\right)^{2} \mathrm{~V}_{\mathrm{m}}^{2} \\
& \rho=\frac{\mathrm{b}}{\mathrm{V}}=-0.2 \cdot 10^{10} \mathrm{~cm}^{-2} \\
& \rho_{\mathrm{s}}=\frac{\mathrm{b}_{\mathrm{s}}}{\mathrm{V}_{\mathrm{s}}}=6.4 \cdot 10^{10} \mathrm{~cm}^{-2}
\end{aligned}
$$

where $\rho$ and $\rho_{s}$ are the scattering densities of micelles and solvent $\left(D_{2} O\right), n$ is the number density of micelles and $V_{m}$ is the volume of a micelle.

The scattering density of micelles is negligible compared to scattering density of solvent $\left(D_{2} O\right)$, so the equation of $I(0)$ is reduced to,

$$
\mathrm{I}(0)=\mathrm{n}\left(\rho_{\mathrm{s}}\right)^{2} \mathrm{~V}_{\mathrm{m}}^{2}
$$

It was known that the volume of the solvent $\left(D_{2} O\right)$ decreases by about $5 \%$ at 1000 bar pressure. Hence, the ratio of $\mathrm{I}(0)$ at 1000 bar and at 100 bar pressures

$$
\frac{\mathbf{I}(0)_{1000 \text { bar }}}{\mathbf{I}(0)_{100 \mathrm{bar}}}=\left(\frac{\mathbf{V}_{\mathrm{D} 2 \mathrm{O}}(1000 \text { bar })}{\mathbf{V}_{\mathrm{D} 20 \mathrm{O}}(100 \text { bar })}\right)^{2}=1.05^{2}=1.1
$$

is

The ratio of experimental $\mathrm{I}(0)$ at 1000 bar and at 100 bar pressure is,

$$
\frac{\mathbf{I}(0)_{1000 \mathrm{bar}}}{\mathbf{I}(0)_{100 \mathrm{bar}}}=\frac{8.989}{8.25}=1.09
$$




\section{Appendix 2}

\section{Activity coefficients of micellar solutions:}

Assumptions:

no salt is incorporated in the solid

$>$ monomers in solution are neglected

chemical potential of surfactant molecules within the solid is changed by variations of $\mathrm{T}$ and $\mathrm{p}$ according to:

$$
d \mu^{(s)}=-\bar{S}^{(s)} d T+\bar{V}^{(s)} d p
$$

in the micellar state, the chemical potential depends also on the concentration of salt:

$$
\begin{aligned}
\mu^{(I)}\left(T, p, C_{N a B r}\right) & =\mu^{o}(T, p)+R T \ln a\left(T, p, C_{N a B r}\right) \\
& =\mu^{\circ}(T, p)+R T \ln \left[\gamma\left(T, p, C_{N a B r}\right) \frac{C_{T T A B}}{C_{o}}\right] \\
& =\mu^{\circ}(T, p)+R T \ln \frac{C_{T T A B}}{C_{o}}+R T \ln \left[\gamma\left(T, p, C_{N a B r}\right)\right]
\end{aligned}
$$

via the activity coefficient $\gamma\left(T, p, C_{N a B r}\right) . \mu^{\circ}(T, p)$ is the chemical potential of a TTAB solution with concentration $\mathrm{c}_{\mathrm{o}}(=1 \mathrm{M})$ without salt.

Hence, $\mu^{(I)}$ varies with $\mathrm{T}, \mathrm{p}$ and $\mathrm{C}_{\mathrm{NaBr}}$ according to:

$$
\mathrm{d} \mu^{(1)}=-\overline{\mathrm{S}}^{(\mathrm{I})} \mathrm{dT}+\overline{\mathrm{V}}^{(1)} \mathrm{dp}+\mathrm{RT} \frac{\partial \ln \gamma}{\partial \mathrm{c}_{\mathrm{NaBr}}} \mathrm{dc} \mathrm{c}_{\mathrm{NaBr}}
$$


here, $\bar{S}^{(1)}$ and $\bar{V}^{(1)}$ are the partial molar entropy and the partial molar volume of the liquid electrolyte solution.

At constant temperature along the equilibrium line in $\mathrm{p}-\mathrm{C}_{\mathrm{NaBr}}$ plane the following equation holds (and this is just the same as in the derivation of ClausiusClapeyrons equation):

$$
\begin{aligned}
\mathrm{d} \mu^{(\mathrm{s})} & =\mathrm{d} \mu^{(l)} \\
& \Rightarrow-\overline{\mathrm{S}}^{(\mathrm{s})} \mathrm{dT}+\overline{\mathrm{V}}^{(\mathrm{s})} \mathrm{dp}=-\overline{\mathrm{S}}^{(1)} \mathrm{dT}+\overline{\mathrm{V}}^{(1)} \mathrm{dp}+\mathrm{RT} \frac{\partial \ln \gamma}{\partial \mathrm{c}_{\mathrm{NaBr}}} \mathrm{dc}_{\mathrm{NaBr}} \\
& \stackrel{\mathrm{T}=\mathrm{const}}{\Rightarrow} \overline{\mathrm{V}}^{(\mathrm{s})} \mathrm{dp}=\overline{\mathrm{V}}^{(1)} \mathrm{dp}+\mathrm{RT} \frac{\partial \ln \gamma}{\partial \mathrm{c}_{\mathrm{NaBr}}} \mathrm{dc}_{\mathrm{NaBr}} \\
& \Rightarrow \frac{\mathrm{dp}}{\mathrm{dc}_{\mathrm{NaBr}}}=-\frac{\mathrm{RT}}{\overline{\mathrm{V}}^{(1)}-\overline{\mathrm{V}}^{(\mathrm{s})}} \frac{\partial \ln \gamma}{\partial \mathrm{c}_{\mathrm{NaBr}}}=-\frac{\mathrm{RT}}{\Delta \overline{\mathrm{V}}} \frac{\partial \ln \gamma}{\partial \mathrm{c}_{\mathrm{NaBr}}}
\end{aligned}
$$




\section{Appendix 3}

\section{X-ray diffraction data:}

X-ray diffraction measurements are carried out for incubated OTAB samples [Carried out with the support of Dr. Klein, Dept. of Crystallography, University of Göttingen]. The diffraction pattern shows the 001 reflections as shown in Figure 4.48. Braggs equation give the distance between the bilayers of surfactant assemblies in solid/coagel phase;

$$
d=\lambda / 2 \sin \Theta
$$

where $d$ is the distance, $\lambda=1.54 \AA(\mathrm{Cu} \mathrm{K} \alpha)$ is the wavelength of the source. The peak around $2 \Theta \approx 3.05$ has used to calculate the $\mathrm{d}$ spacing and found to be around $29 \AA$ which is almost equal to the length of the surfactant molecule and the probable picture of the solid would be the bilayers of interdigitated surfactants.

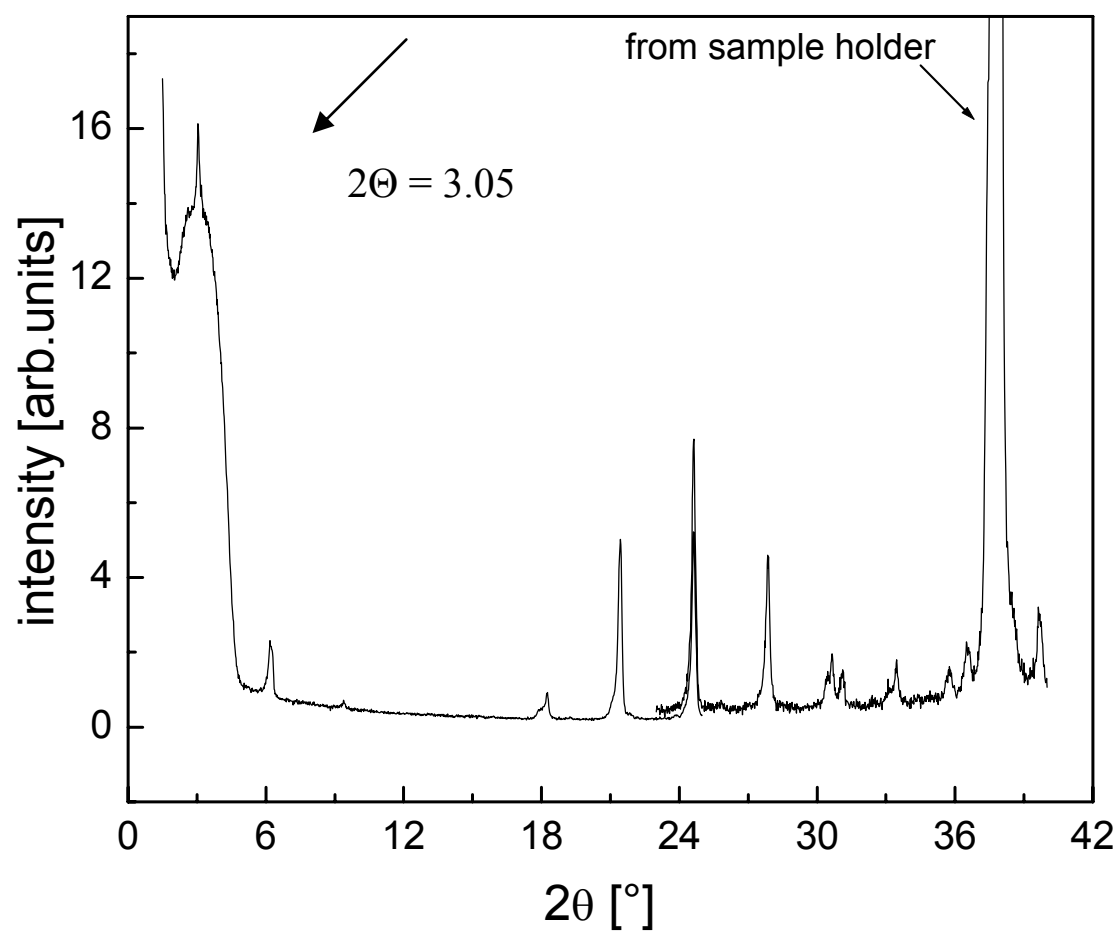

Figure 4. 48: X-ray diffraction pattern of solid OTAB (incubated sample) measured at $25^{\circ} \mathrm{C}$ 


\section{Appendix 4}

On cooling, around $5{ }^{\circ} \mathrm{C}$ micellar solution $0.4 \mathrm{M}$ TTAB turn to solid phase. Figure 4.49 presents Raman spectra at $4{ }^{\circ} \mathrm{C}$ and $2{ }^{\circ} \mathrm{C}$ along with a picture of the solid TTAB coexisting with clear liquid. In Figure $4.49 \mathrm{~b}$, Spectrum at $4{ }^{\circ} \mathrm{C}$ shows the presence of water with raising intensity near $3100 \mathrm{~cm}^{-1}$ (which is the starting point of the water peak around $3450 \mathrm{~cm}^{-1}$ ), and much reduced intensity in spectrum recorded at $2{ }^{\circ} \mathrm{C}$. This suggests that the water is present along with the solid first and then most of the water will squeezes out of the solid. In Figure 4.49a, the blue arrow indicates solid portion and that of green arrow indicates the clear liquid.
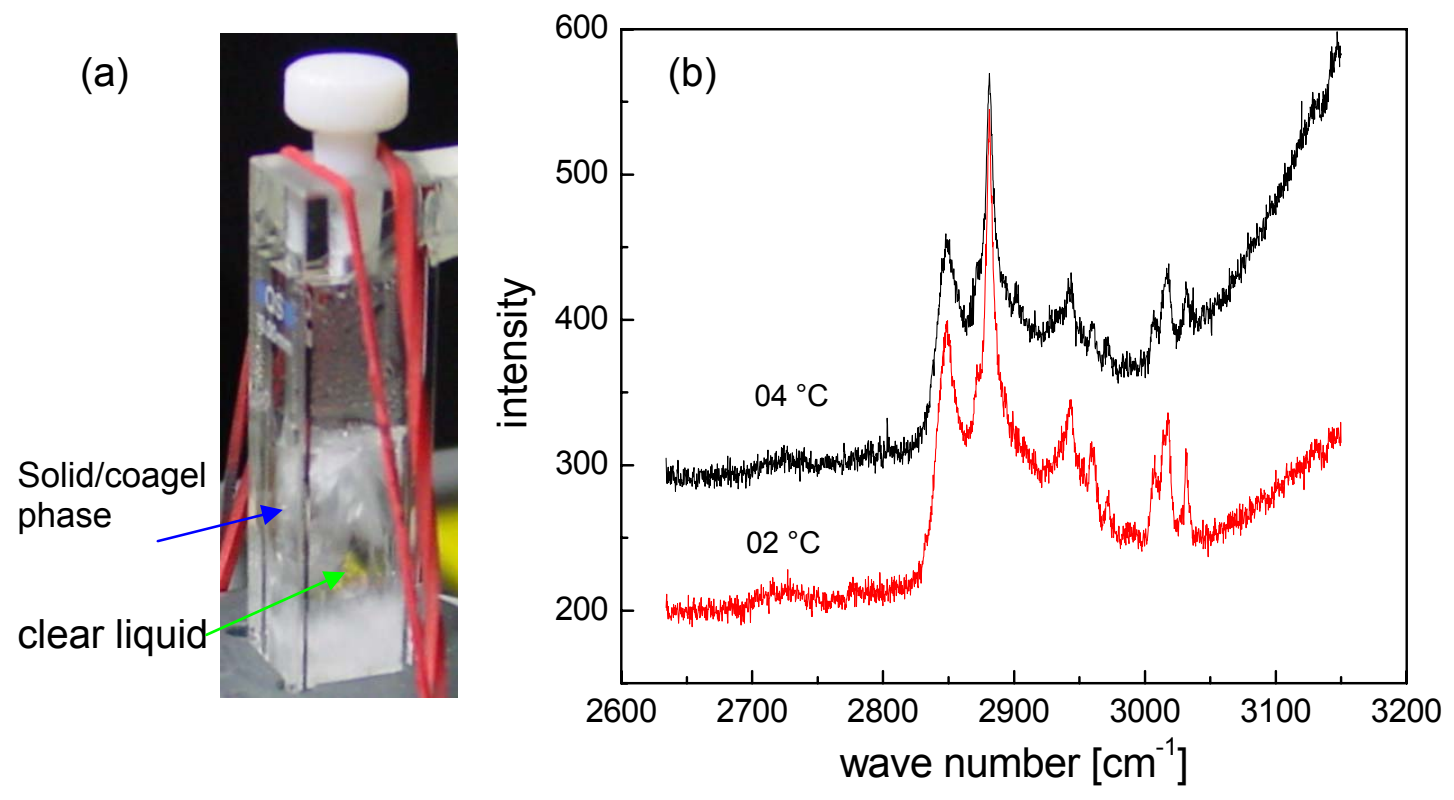

Figure 4. 49: (a) Picture of TTAB coexisting with water (red colored rubber band is as a support for the cuvette), (b) representative Raman spectra at 4 and $2{ }^{\circ} \mathrm{C}$ for 400 mM TTAB 


\section{Literature}

Abbott_1979: R. J. Abbott and D. W. Oxtoby J. Chem. Phys. 1979, 70, 4703

Akutsu_1981: H. Akutsu, Biochemistry 1981, 20, 7359

Ambrosi_2004: M. Ambrosi, P. Lo Nostro, L. Frantoni, L. Dei, B. W. Ninham, S.

Pălma, R. H. Manzo, D. Allemandi and P. Baglioni, Phys. Chem. Chem. Phys 2004, 6, 1401

Amorim_1982: A. M. Amorim Da Costa, C. F. G. C. Geraldes, and J. J. C. Teixeira-Dias, J. Colloid Interface Sci. 1982, 86, 254

Aswal_2000: V. K. Aswal and P. S. Goyal, Phys. Rev. E, 2000, 61, 2947

Aswal_2002: V.K. Aswal, P.S. Goyal, Chemical Physics Letters 2002, 364, 44

Aswal_2003: V. K. Aswal and P. S. Goyal, Phys. Rev. E, 2003, 67, 051401

Baden_2002: N. Baden, O. Kajimoto, and K. Hara, J. Phys. Chem. B 2002, 106, 8621

Balzaretti_2003: N. M. Balzaretti, C. A. Perottoni, and J. A. H. da Jornada, J. Raman Spectrosc. 2003, 34, 259

Barnes_1972: J. D. Barnes and B. M. Fanconi, J. Chem. Phys. 1972, 56, 5190

Bartoli_1972: F. J. Bartoli and T. A. Litovitz, J Chem. Phys. 1972, 56, 404

Bendedouch_1983: D. Bendedouch, S. H. Chen and and W. C.

Koehler, J. Phys. Chem. 1983, 87, 2621

Bergström_1999: M. Bergstrom and J. S. Pedersen, Phys. Chem.

Chem. Phys., 1999, 1, 4437

Blandamer_1995: M. J . Blandamer, P. M . Cullis, L . Giorgiosoldi, Jan B. F.N. Engberts, A. Kacperska, N. M. Van os. M.C.S. Subha, Adva. Colloid. Inter. Sci. 1995, 58, 171

Boerio_1970: F. J. Boerio and J. L. Koenig, J. Chem. Phys. 1970, 52, 3425

Bossev_2001: D. P. Bossev, S. R. Kline, J. N. Israelachvili, and M. E. Paulaitis, Langmuir 2001, 17, 7728 
Brown_1987: K. G. Brown, E. B. Brown, and M. Ladjadj, J. Phys. Chem. 1987, $\overline{9} 1,3436$

Buback_1994: M. Buback, in Application of high-pressure techniques in: Infrared and Raman Spectroscopy-Methods and Applications, ed. B. Schrader, VCH Verlag, Weinheim, 1994, p. 640

Cameron_1980: David G. Cameron, Hector L. Casal, Eva F. Gudgin, and Henry H. Mantsch, Biochimica et Biophysica Acta 1980, 596, 463

Casal_1982: H. L Casal, H. H. Mantsch and D. G. Cameron, J Chem. Phys 1982, 77,2825

Casal_1984: H. L. Casal, and H. H. Mantsch, Biochimica et Biophysica Acta 1984, 779, 381

Chen_1986: S. H. Chen, Ann. Rev. Phys. Chem. 1986, 37, 351

Chen_1988: S. H. Chen, E. Y. Sheu, J. Kalus and H. Hoffmann, J. Appl. Crystallogr. 1988, 21, 751

Corrin_1947: M. L. Corrin, D. W. Harkin, J. Am. Chem. Soc., 1947, 69, 679

Domínguez_1997: A. Domínguez, A. Fernández, N. González, E. Iglesias, and Luis Montenegro, J. Chem. Educ. 1997, 74, 1227

Doscher_1951: T. M. Doscher, G. E. Myers and Jr. D. C. Atkins, J. Colloid Sci. 1951, 6, 223

Eckold_2001: G. Eckold, N. Gorski, Colloids Surf., A, 2001, 183-185, 361

Evans_1956: H. C. Evans, J. Chem. Soc. 1956, 579

Fan_1997: A. Fan, P. Somasundaran, and N. J. Turro, Langmuir 1997, 13, 506

Fisch_1986: M. R. Fisch and G. B. Benedek, J. Chem. Phys. 1986, 85, 553

Gaber_1977: B. P. Gaber and W. P. Petocolas, Biochimica et Biophysica Acta 1977, 465, 260

Gardiner_1989: D. J. Gardiner and P. R. Graves, Practical Raman Spectroscopy 1989, Springer Verlag, Berlin

Gharibi_1998: H. Gharibi, A. A. Rafati, A. Feizollahi, B. M. Razavizadeh, M.A. Safarpour, Colloids Surfaces A, 1998, 145, 47

Goddard_1953: E. D. Goddard, O. Harva, and T. G. Jones, Trans. Faraday. Soc. 
$1953,49,980$

Gorski_1994:N. Gorski, M. Gradzielski, and H. Hoffmann, Langmuir, 1994, 10, 2594

Gorski_1997: N. Gorski, J. Kalus, A. I. Kuklin and L. S. Smirnov, J. Appl. Cryst. 1997, 30, 739

Gorski_1999: N. Gorski, J. Kalus, and D. Schwahn, Langmuir 1999, 15, 8080

Gorski_2001: N. Gorski and J. Kalus, Langmuir 2001, 17, 4211

Haldar_2001: J. Haldar, V. K. Aswal, P. S. Goyal, and S. Bhattacharya, Angew. Chem. Int. Ed. 2001, 40, 1228

Hamley_2000: I. W. Hamley, Introduction to Soft Matter 2000 (Polymer, Colloids, Amphiphiles and Liquid Crystals), John Wiley \& Sons, Chichester, England

Hartley_1936: G. S. Hartley, Aqueous Solutions of Paraffin-Chain Salts 1936 Hermann \& Cie., Paris

Hattori_1999: N. Hattori, M. Hara, H. Okabayashi, C.J. O'Conno, Colloid Polym Sci 1999, 277, 306

Hayter_1981: J. B. Hayter and J. Penfold, Mol. Phys., 1981, 42, 109

Heenan: R. Heenan, http://www.isis.rl.ac.uk/largescale/loq/loq.htm

Herzfeld_1952: S. H. Herzfeld, J. Phys. Chem 1952, 56, 959

Hoffmann_1983: H. Hoffmann, J. Kalus, H. Thurn and K. Ibel, Ber. Bunsenges. Phys. Chem., 1983, 87, 1120

Huang_1982: C-hsien Huang, J. R. Lapides, and I. W. Levin, J. Am. Chem. SOC. 1982, 104, 5926

Imae_1986: T. Imae and S. Ikeda, J. Phys. Chem. 1986, 90, 5216

Kalyanasundaram_1976: K. Kalyanasundaram and J. K Thomas, J. Phys. Chem 1976, $80,1 \overline{4} 62$

Kaneshina_1990: Shoji Kaneshina and Michio Yamanaka, J. Colloid Interface Sci. $1990,140,474$ 
Kawai_1985: T. Kawai, J. Umemura, T. Takenaka, M. Kodama, and S. Seki, J. Colloid Interface Sci. 1983, 103, 56

Klevens_1953: H. B. Klevens, J. Am. Oil Chemists' Soc. 1953, 30, 74

Kobayashi_1980: M. Kobayashi, H. Tadokoro, R. S. Porter J.Chem.Phys. 1980, $73, \overline{3} 635$

Kodama_1986: M. Kodama and S. Seki, J. Colloid Interface Sci. 1986, 117, 487 Kuklin_2005: A. I Kuklin, A. Kh. Lsamov and V. I. Gordeliy, Neutron News, 2005 16,9

Kumar_2000: S. Kumar, D. Sharma, and Kabir-ud-Din, Langmuir 2000, 16, 6821

Laughlin_1994: R. G. Laughlin, 1994, The Aqueous Phase Behavior of Surfactants, Academic Press, London

Levin_1981: I. W. Levin and S. Fowler Bush Biochimica et Biophysica Acta 1981, 640,760

Lewis_2001: I. R. Lewis and Howell G M Edwards, Handbook of Raman Spectroscopy 2001, Marcel Dekker, New York

Lippert_1971: J. L. Lippert and W. L. Peticolas, Proc. Nat. Acad. Sci. 1971, 68, 1572

Liu_2006: Q. Liua, X. Lia, L. Hao, C. Tan, Y. Zhoub, P. Chen, H. Zhu, Chin. J. Chem. Pyhs. 19, 2006, 15

Maayouf_1994: R. M. A. Maayouf, V. L. Aksenov, M. A. Kiselev, A. I. Kuklin, I. N. Serdyuk, A. S. Smirnov, JINR Commun. 1994, E13-94-257

McCreery_2000: R. L. McCreery, Raman Spectroscopy for Chemical Analysis, 2000 John Wiley \& Sons, New York

Mantsch_1982: H. H. Mantsch, D. G. Cameron, P. A. Trembley and M. Kates, Biochimica et Biophysica Acta 1982, 689, 63

Mazer_1976: N. A. Mazer, G. B. Benedek, and M. C. Carey, J.Phys. Chem. 1976, 80, 1075

McBain_1913: J. W. Mc Bain, Trans. Faraday Soc. 1913, 9, 99

Mizushima_1949: S. I. Mizushima and T. Simanouti, J. Am. Chem. Soc. 1949, $71,1 \overline{320}$

Nakagawa_1963: K. Shinoda, T. Nakagawa, Bun-Ichi Tamamushi, Isemura, 
Colloidal Surfactants 1963, Academic Press, London,

Nicoli_1979: D. F. Nicoli, and D. R. Dawson and H. W. Offen, Chem. Phys. Lett. 1979, 66, 291

Offen_1980: H. W. Offen, Rev. Phys. Chem. Japn. 1980, 85, 553

Offen_1982: H. W. Offen and W. D. Turley, J. Colloid Interface. Sci. 1982, 87, 442

Ornstein_1914: L. S. Ornstein and F. Zernicke, Proc. K. Ned. Akad. Wet., 1914 $1 \overline{7}, 793$

Ostanevich_1988: Y. M. Ostanevich, Makromol. Chem., Macromol. Symp., 1988, 15, 91

Patrick_1999: H. N. Patrick, G. G. Warr, S. Manne, and I. A. Aksay, Langmuir 1999, 15, 1685

Picquart_1986: Michael Picquart, J. Phys. Chem. 1986, 90, 243

Prathima_2005: N. Prathima, M. Harini, Neeraj Rai, R. H. Chandrashekara, K. G. Ayappa, S. Sampath, and S. K. Biswas, Langmuir 2005, 21, 2364

Raghavan_1975: V. Raghavan and M. Cohen, in Treatise on Solid State Chemistry, ed. A. Hannay, Plenum, New York, 1975, vol. 5, p. 67

Rubingh_1991: D. N Rubingh and P. M Holland, 1991, Cationic SurfactantsPhysical Chemistry (surfactant science series volume 37), Marcel Dekker, New York

Schoen_1979: P. E. Schoen, R. G. Priest, J. P. Sheridan and J. M. Schnur, J. Chem. Phys. 1979, 71, 317

Simanouti_1949: T. Simanouti and S. I. Mizushima, J Chem.Phys. 1949, 17, 1102

Shaufele_1967: R. F. Shaufele and T Shimanouchi, J. Chem. Phys. 1967, 47, 3605

Shinoda_1955: K. Shinoda, J. Phys. Chem. 1955, 432

Shinoda_1963: K. Shinoda, T. Nakagawa, Bun-Ichi Tamamushi, Isemura, Colloidal Surfactants 1963, Academic Press, London,

Silva_2003: Manuela Ramos Silva, Ana Matos Beja and Jose Antonio Paixão, 
Acta Cryst. 2003, E59, 01151

Snyder_1960: R. G. Snyder, J. Mol. Spectrosc. 1960, 4, 411

Snyder_1961: R. G. Snyder, J. Mol. Spectrosc. 1961, 7, 116

Snyder_1963: R. G. Snyder, Spectrochim. Acta. 1963, 19, 85

Snyder_1963: R. G. Snyder, Spectrochim. Acta. 1963, 19, 117

Snyder_1967: R. G. Snyder, J. Chem Phys. 1967, 47, 1316

Snyder_1978: R. G. Snyder, S. L. Hsut and S. Krimm, Spectrochim. Acta 1978, $34 \mathrm{~A}, 395$

Snyder_1979: R. G. Snyder J. Chem. Phys. 1979, 71, 3229

Snyder_1980: R. G. Snyder, J. R. Scherer, B. P. Gaber, Biochimica et Biophysica Acta 1980, 601, 47

Snyder_1982: R. G. Snyder, D. G. Cameron, H. L. Casal, D. A. C. Compton, and H. H. Mantsch, Biochimica et Biophysica Acta 1982, 684, 111

Snyder_1982a: R. G. Snyder, H. L. Strauss and and C. A. Elllger J. Phys. Chem. $1982,86,5145$

Stodghill_2004: S. P. Stodghill, A. E. Smith, and J. H. O'Haver, Langmuir 2004, 20,11387

Susi_1980: H. Susi, D.M. Byler and W.C. Damert, Chemistry and Physics of Lipids, 1986, 27, 337

Tanaka_1973: M. Tanaka, S. Kaneshina, Tahei Tomida, K. Noda and K. Aoki, J. Colloid Interface Sci. 1973, 44, 525

Tanford_1972: C. Tanford, J. Phys. Chem 1972, 76, 3020

Tanford_1973: C. Tanford, 1973, The Hydrophobic Effect, John Wiley \& Sons, New York

Tarter_1955: H. V. Tarter, J. Phys. Chem 1955, 59, 1195-1199

Tasumi_1962: M. Tasumi, T. Shimanouchi and T. Miyazawa, J. Mol. Spectrosco 1962, 9, 261 
Tsuchiya_1994: M. Tsuchiya, K. Tsujii, K. Maki and T. Tanaka, J. Phys. Chem. 1994, 98, 6187-6194

Vogel_1981: H. Vogel and F. Jähnig, Chemistry and Physics of Lipids, 1981, 29, 83

Wang_2004: X. Wang, J. Wang, Y. Wang, and H. Yan, Langmuir 2004, 20, 53

Warr_1994: H. N. Patrick, G. G. Warr, S. Manne, and I. A. Aksay, Langmuir 1999, 15, 1685

Widjaja_2005: N. Widjaja, 2005, Department of Physics, University of Tennessee, Knoxville, TN 37996

Wilson_1955: E B Wilson (Jr.), J C Decius, Paul C Cross, Molecular Vibrations 1955, McGraw- Hill, New York

Winter_1989: R. Winter and W. C. Pilgrim, Ber. Bunsen-Ges. Phys. Chem., 1989, 93, 708

Woolley_1985: E. M. Woolley, and T. E. Burchfield, J. Phys. Chem. 1985, 89, $7 \overline{14}$

Wong_1982: P. T. T. Wong, W. F. Murphy, and H. Mantsch, J. Chem. Phy. 1982, 76,5230

Wong_1983: P. T. T. Wong and H. H. Mantsch, J. Chem. Phys. 1983, 78, 7362

Wong_1983a: P. T. T. Wong, and H. Mantsch, J. Phys. Chem. 1983, 87, 2436

Wong_1983b: P. T. T. Wong, and H. Mantsch, Biochimica et Biophysica Acta 1983, 732, 92

Wong_1984: P. T. T Wong, Ann. Rev. Biophys. Bioeng. 1984, 13, 1

Wong_1985: P. T. T. Wong and H. H. Mantsch, J. Chem. Phys. 1985, 83, 3268

Wong_1985a: P. T. T. Wong and H. H. Mantsch, Biochemistry 1985, 24, 4091

Wong_1985b: P. T. T. Wong, E. Mushayakarara and H. H. Mantsch, J. Raman Spectrosc. 1985, 16, 427

Wong_1986: P. T. T. Wong, and H. Mantsch, J. Raman Spectrosc. 1986, 17, 335

Wong_1987: P. T. T. Wong, T. E. Chagwedera and H. H. Mantsch, J. Chem.

Phys. 1978, 87, 4487 
Wu_1982: W.-guey Wu, C.-hsien Huang, T. G. Conley, R. B. Martin, and I. W. Levin, Biochemistry, 1982, 21, 5957

Yagui_2005: C. O. Rangel-Yagui, H. Wei Ling Hsu, A. Pessoa- Jr, L. C. Tavares, Brazilian Journal of Pharmaceutical Sciences, 2005, 41, 237

Yamaguchi_2003: M. Yamaguchi, S. V. Serafin, T. H. Morton, and E. L. Chronister, J. Phys. Chem. B 2003, 107, 1815

Yang_1986: P. W. Yang, H. H. Mantsch, J. Colloid Interface Sci. 1986, 113, 218

Yelli_1977: N. Yellin and I. W. Levin, Biochemistry 1977, 16, 462

Zana_1983: R. Zana, C. Picot and R. Duplessix, J. Colloid Interface Sci. 1983, 94,43

Zielinski 1987: R. Zielinski, S. Ikeda, H. Nomura and S. Kato, J. Colloid Interface Sci. 1987, 119, 398 


\section{List of Figures and tables}

Figure 2. 1: Schematic representation of an amphiphile, the part which is inside the dotted oval is hydrophilic (head) group and a counter ion, and the rest hydrophobic part.

Figure 2. 2 : The Mc Bain lamellar micelles The Hartley spherical micelles. 12

Figure 2. 3: Schematic representation of the models of prolate (i) and oblate (ii) shperoids, 'a', the major semi-axis and the 'b', the minor semi-axis

Figure 2. 4: Schematic illustrations of the rotational state of surfactant molecules in both the coagel and the gel phase. Open circles and filled circles denote hydrogen atoms and oxygen atoms, respectively, in the cross sectional view (taken from Tsuchiya_1994)

Figure 2. 5: Phase diagram of ionic surfactant 22

Figure 3. 1: cmc of DTAB determined by conductivity measurements (red lines are linear fit) 24

Figure 3. 2: Molar conductance against square root of concentration (inset is the expanded part of lower $\mathrm{x}$-axis scale and red line is linear fit) 24

Figure 3. 3: Possibilities of scattering of light; the Rayleigh scattering, stokes (molecules absorb enegry) and anti stokes (molecule release energy) Raman scatterings are shown.... 25

Figure 3. 4: Traditional Raman scattering geometry .........................................29

Figure 3. 5: Jobin-Yvon T64000 triple monochromator Raman spectrometer 31

Figure 3. 6: Representative Raman spectra for powder TTAB and $0.4 \mathrm{M}$ aqueous micellar solution, (a) below $600 \mathrm{~cm}^{-1}$ longitudinal accordion modes, (b) C-C skeletal and $\mathrm{CH}_{2} / \mathrm{CH}_{3}$ bending modes, (c) $\mathrm{CH}_{2} / \mathrm{CH}_{3}$ stretching modes 33

Figure 3. 7: Schematics of the vibrations of alkane (adapted from Simanouti_1949, Schaufele_1967). 34

Figure 3. 8: Polarization measurements for $0.4 \mathrm{M}$ aqueous TTAB micellar solution at ambient conditions. Part "a" corresponds to the $z z$ and zy polarized spectra and "b" the expanded part of "a" in $\mathrm{CH}_{2}$ stretching region for better view and spectrums are shifted vertically for clarity 36

Figure 3. 9: Comparison of isotropic part of the $z z$ polarzed spectra with depolarized spectra 
Figure 3. 10: Interaction of beams of neutrons, X-rays, and electrons with material 38

Figure 3. 11: Schematic view of the YuMO spectrometer at IBR 2, Dubna, Russia .40

Figure 3. 12: Schematic representation of SANS scattering geometry, the terms used in the figures are described in the text.

Figure 3. 13: Schematic illustration of isotopic effect in SANS study

Figure 3. 14: SANS intensity profile of $25 \mathrm{mM}$ TTAB in $\mathrm{D}_{2} \mathrm{O},(+)$ are experimental, the solid line for form factor and the dashed line is for structure factor from the fits of Hayter-Penfold model 45

Figure 3. 15: (a) Pressure cell used for Raman spectra measurements, (b) Pressure cell used for SANS measurements 46

Figure 3. 16: Schematic representation of TTAB surfactant molecule, red, dark, green and magenta colored balls represent hydrogen, carbon, nitrogen and bromide atoms respectively.... 48

Figure 4. 1: Critical micelle concentration $(\mathrm{cmc})$ of TTAB determination by conductivity measurement 49

Figure 4. 2: Raman spectrum of $0.4 \mathrm{M}$ TTAB in water, (a) longitudinal accordion modes (below $300 \mathrm{~cm}^{-1}$ ). The intensity of the spectral region $300 \mathrm{~cm}^{-1}$ to $950 \mathrm{~cm}^{-1}$ is enhanced by a factor of 3 for better peak resolution,(b) $850 \mathrm{~cm}^{-1}$ to $1500 \mathrm{~cm}^{-1}$ corresponds to skeletal deformations 50

Figure 4. 3: SANS intensity profile of $25 \mathrm{mM}$ TTAB in $\mathrm{D}_{2} \mathrm{O},(+)$ are experimental, the solid line for form factor and the dashed line is for structure factor from the fits of Hayter-Penfold model 52

Figure 4. 4: Representative SANS intensity profiles at different TTAB concentration .52

Figure 4. 5: Concentration dependence of the long micelle-axis $\mathrm{c}$ and the

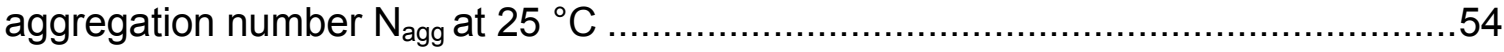

Figure 4. 6: Concentration dependence of the correlation peak position $Q_{\max } \ldots \ldots . . . .55$

Figure 4. 7a: Changes in the shape of the Raman spectra of $0.4 \mathrm{M}$ TTAB with temperature (spectra are shifted vertically for clarity).............................................56

Figure 4. 7b: Changes in the shape of the Raman spectra of $0.4 \mathrm{M}$ TTAB with

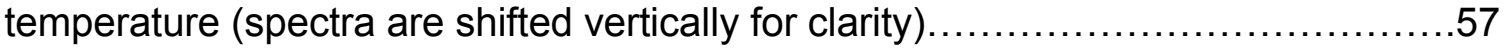

Figure 4. 7c: Changes in the shape of the Raman spectra of $0.4 \mathrm{M}$ TTAB with temperature (spectra are shifted vertically for clarity) ...............................58 
Figure 4. 8: (a) SANS intensity profiles for $50 \mathrm{mM}$ TTAB in $\mathrm{D}_{2} \mathrm{O}$ at 3 different temperatures, and (b) aggregation number of $25 \mathrm{mM}$ and $50 \mathrm{mM}$ TTAB at different temperatures 59

Figure 4. 9a: Longitudinal accordian modes at $20{ }^{\circ} \mathrm{C}$ and $02{ }^{\circ} \mathrm{C}$ and comparison with the powder TTAB measured at room temperature

Figure 4. 9b: $\mathrm{C}-\mathrm{H}$ deformation and $\mathrm{C}-\mathrm{C}$ stretching modes at $20{ }^{\circ} \mathrm{C}$ and $02{ }^{\circ} \mathrm{C}$ and comparison with the powder TTAB measured at room temperature.

Figure 4. 9c: $\mathrm{C}-\mathrm{H}$ stretchning at $20^{\circ} \mathrm{C}$ and $02{ }^{\circ} \mathrm{C}$ and comparison with the powder TTAB measured at room temperature.

Figure 4. 10: Frequency shifts of $0.4 \mathrm{M}$ TTAB as a function of temperature $\mathrm{CH}_{2}$ twisting mode

Figure 4. 11: Full width half maxima as a function of temperature (a) $\mathrm{C}-\mathrm{C}$ stretching 64

Figure 4. 12: Peak frequency differences as a function of temperature (a) symmetric $\left(1123 \mathrm{~cm}^{-1}\right)$ and asymmetric $\left(1064 \mathrm{~cm}^{-1}\right) \mathrm{C}-\mathrm{C}$ stretch (b) $1140 \mathrm{~cm}^{-1}$ and $1123 \mathrm{~cm}^{-1} .65$

Figure 4. 13: Ratios peak height of trans to gauche in C-C stretching region (symbols are described in text) 66

Figure 4. 14: Changes in the methylene asymmteric $\mathrm{C}-\mathrm{H}$ stretching band with temperature, measurements done in zy polarization mode for $0.4 \mathrm{M} \mathrm{TTAB}$ 67

Figure 4. 15: Changes in $\mathrm{C}-\mathrm{H}$ stretching regime (a) shift in peak frequency, (b) ratio of peak height of asymmetric to symmetric bands (solid lines are to guide the eye) 68

Figure 4. 16: Representative SANS intensity profiles for $300 \mathrm{mM}$ TTAB solution in $\mathrm{D}_{2} \mathrm{O}$, at $7{ }^{\circ} \mathrm{C}$ solidification observed. 72

Figure 4. 17: Pressure dependence of Raman spectra for $50 \mathrm{mM}$ TTAB-solution at $25^{\circ} \mathrm{C}$ in the range of $\mathrm{C}-\mathrm{C}$ skeleton and $\mathrm{C}-\mathrm{H}$ bending vibrations. The two spectra at 1100 bar correspond to subsequent runs during phase transition. For comparison, the spectrum of dry TTAB-powder is shown in the upper part 73

Figure 4. 18: (a) Pressure dependence of the intensity ratio of trans and gauche bands at $1123 \mathrm{~cm}^{-1}$ and $1140 \mathrm{~cm}^{-1}$ (The line is drawn to guide the eye.), (b) pressure dependence of the peak separation of trans and gauche-bands at $1123 \mathrm{~cm}^{-1}$ and $1140 \mathrm{~cm}^{-1}$ 75

Figure 4. 19: (a) Small angle neutron scattering from $25 \mathrm{mM}$ TTAB-solution at $25^{\circ} \mathrm{C}$ at different pressures. The lines are results from a fit to the Hayter-Penfold model, (b) time evolution of scattering profiles at 1100 bar (in the inset the time dependence 
of the Porod invariant is shown). Note, that the individual curves are shifted vertically for clarity

Figure 4. 20: $p$-T phase diagram of TTAB solution at low concentrations ( $25 \mathrm{mM} / 50$ $\mathrm{mM}$ ) as obtained by SANS and Raman scattering ....

Figure 4. 21: Time evolution of SANS intensity of $360 \mathrm{mM}$ TTAB solution at $25^{\circ} \mathrm{C}$ and 700 bar. In the inset, the time dependence of the Porod invariant is shown - for comparison the data for 900 bar are also included 80

Figure 4. 22: Pressure-concentration phase diagram of TTAB at $25^{\circ} \mathrm{C}$. The 82

Figure 4. 23: Raman spectra of $50 \mathrm{mM}$ TTAB with and without electrolyte, $\mathrm{NaBr}$, in C-C stretching region (the spectra are shifted vertically for clarity) 85

Figure 4. 24: Raman spectra in $\mathrm{C}-\mathrm{H}$ stretching region of methylene, and methyl groups of head group. Dashed vertical lines are given to follow the peak position (spectrums are shifted vertically for clarity) 86

Figure 4. 25: Shift in peak frequency $\left(3036 \mathrm{~cm}^{-1}\right)$ with addition of electrolyte (the deviation of a data point at $2 \mathrm{M}(\mathrm{zy})$ might be an artifact of the experiment).... 87

Figure 4. 26: Variation of small angle scattering intensity profiles of a $50 \mathrm{mM}$ TTAB solution with increasing $\mathrm{NaBr}$ concentration at $25^{\circ} \mathrm{C}$. 88

Figure 4. 27: Evolution of Raman spectra in the region of $\mathrm{CH}_{3} / \mathrm{CH}_{2}$ bending vibrations at $25^{\circ} \mathrm{C}$ during crystallization at about 950 bar. (Note that the individual spectra are shifted in vertically for clarity.). 89

Figure 4. 28: Time-evolution of small angle scattering of $50 \mathrm{mM}-\mathrm{TTAB}$ with $0.1 \mathrm{M}$ $\mathrm{NaBr}$ at $30^{\circ} \mathrm{C}$ after increasing the pressure to 1000 bar. 90

Figure 4. 29: Pressure-temperature phase diagram of $50 \mathrm{mM}$ TTAB with different concentrations of $\mathrm{NaBr}$

Figure 4. 30: Influence of electrolyte; variation of phase transition pressure at $25{ }^{\circ} \mathrm{C}$

Figure 4. 31: Variation of crystallization temperature at 1 bar as a function of $\mathrm{NaBr}-$ concentration 93

Figure 4. 32: (a) Comparison of Raman spectra for powder sample at low frequency regime (LAM), (b) the shift in peak frequency LAM with alky chain length. (Note that the individual spectra are shifted vertically for clarity.) 96

Figure 4. 33: (a) Comparison of Raman spectra for powder sample, (b) the peak frequency difference of symmetric and asymmetric $\mathrm{C}-\mathrm{C}$ stretching and shift in gauche band $\left(1090 \mathrm{~cm}^{-1}\right)$. (Note that the individual spectra are shifted vertically for clarity.) .96 
Figure 4. 34: Comparison of Raman spectra in micellar phase, OTAB is measured at $40{ }^{\circ} \mathrm{C}$ and all other surfactant are at $25^{\circ} \mathrm{C}$. (Note that the individual spectra are shifted vertically for clarity.) 98

Figure 4. 35: (a) Comparison of Raman spectra in micellar phase showing only in .99

Figure 4. 36: SANS intensity profiles of alkyltrimethylammonium Bromide (C12 to C18) at their $100 \mathrm{mM}$ micellar solution 100

Figure 4. 37: Raman spectra of $100 \mathrm{mM}$ OTAB in C-C stretching, $\mathrm{CH}_{2}$ twisting and $\mathrm{CH}_{2}$ bending regime, the numbers are the temperature in ${ }^{\circ} \mathrm{C}$ at which the spectra are recorded and the arrow indicates direction of the measurements (Individual spectra are shifted for clarity) 102

Figure 4. 38: Raman spectra of $100 \mathrm{mM}$ OTAB in $\mathrm{CH}$ stretching regime, the numbers are the temperature in ${ }^{\circ} \mathrm{C}$ at which the spectra are recorded and the arrow indicates direction of the measurements (Individual spectra are shifted for clarity) 103

Figure 4. 39: Raman spectra of $100 \mathrm{mM}$ OTAB in $\mathrm{C}-\mathrm{C}$ stretching, $\mathrm{CH}_{2} / \mathrm{CH}_{3}$ bending regime, the numbers are the temperature in ${ }^{\circ} \mathrm{C}$ at which the spectra are recorded and the arrow indicates direction of the measurements (Individual spectra are shifted for clarity) 104

Figure 4. 40: Raman spectra of $100 \mathrm{mM}$ OTAB in $\mathrm{C}-\mathrm{H}$ stretching regime, the numbers are the temperature in ${ }^{\circ} \mathrm{C}$ at which the spectra are recorded and the arrow indicates direction of the measurements (Individual spectra are shifted for clarity) 105

Figure 4. 41: Ratio of peak height of trans $\left(1126 \mathrm{~cm}^{-1}\right)$ to gauche $\left(1085 \mathrm{~cm}^{-1}\right)$. Data are from phase transition of micelles to metastable gel phase and from solid (coagel) to micelles. Solid lines are just an eye guide.... 106

Figure 4. 42: Raman spectra at three different phase (micelles, metastable gel and solid (coagel)); in comparison with the photographs of the same (see the text for details). Red bands in the photographs are of rubber band used for tightening the cuvette 107

Figure 4. 43: Ratio of peak height of methylene asymmetric and symmetric $\mathrm{C}-\mathrm{H}$ stretching band. Data are from phase transition of metastable gel phase to micelles and vise versa and from solid (coagel) to micelles. Solid lines are just an eye guide

Figure 4. 44: SANS intensity profiles of $100 \mathrm{mM}$ OTAB, upper part is below $25^{\circ} \mathrm{C}$ (gel phase), bottom part corresponds for micellar phase. Y-axis is different for both parts

Figure 4. 45: $50 \mathrm{mM}$ aqueous micellar solution of HTAB, spectrums measured at 800 bar as a function of time, spectrum with black color is measured at 100 bar. All measurements are at $25^{\circ} \mathrm{C}$ (spectrums are shifted vertically for clarity) 112 
Figure 4. 46: Representative pictures of kinetics of phase transition of $100 \mathrm{mM} \mathrm{HTAB}$ at $05^{\circ} \mathrm{C}$ (red colored rubber band is as a support for the cuvette) 114

Figure 4. 47: Schematic of the illustration of different phases of alkyltrimethylammoinum bromides 114

Figure 4. 48: X-ray diffraction pattern of solid OTAB (incubated sample) measured at $25^{\circ} \mathrm{C}$ 124

Figure 4. 49: (a) Picture of TTAB coexisting with water (red colored rubber band is as a support for the cuvette), (b) representative Raman spectra at 4 and $2{ }^{\circ} \mathrm{C}$ for 400 mM TTAB

125

Table 2. 1: Showing the packing parameters for different aggregates

Table 3. 1: Tentative assignment for vibration bands of tetradecyltrimethylammonium bromide surfactant both in micelles and powder form, assignments are on the basis of literature. All the assignments taken from Kalyanasundaram_1976, unless otherwise referenced against the frequencies. (a) is measured in zy polarization ....35

Table 4.1: Geometrical parameters of TTAB micelles at ambient conditions for different concentrations: $a, b$ and $c$ are the half axes of the ellipsoid, $Z_{o}$ and $N_{\text {agg }}$ are the average charge and the aggregation number, respectively. $\alpha$ is the degree of dissociation and $\chi^{2}$ the mean squared deviation of the fit 53

Table 4. 2: Tentative assignment for vibration bands of tetradecyltrimethylammonium Bromide surfactant in micelles, after phase transition and powder form, assignments are on the basis of literature. All the assignments taken from Kalyanasundaram_1976, unless otherwise referenced against the frequencies. (a) is measured in zy polarization

Table 4. 3: cmc of alkyltrimethyammonium bromides, for HTAB and OTAB the data 95

Table 4. 4: Geometrical parameters of micellar solutions of alkyltrimethylammonium bromides at ambient conditions 101 


\section{Acknowledgements}

I extend my sincere gratitude and appreciations to many people who made this doctoral thesis possible. Special thanks are due to my supervisor Prof. Dr. Götz Eckold for his timely advice and cooperation. Thanks are also due to Dr. Holger Gibhardt and the entire group for their kind cooperation and support in lab work.

I would like to thank Dr. A. Kh. Islamov and A. I. Kuklin, Frank Laboratory of Neutron Physics, Joint Institute for Nuclear Research, Dubna, Russia for helping with SANS experiments and results, and for fruitful discussions.

I would like to thank Prof. Dr. C. Griesinger, Max-Planck Institute of Biophysical Chemistry for being second supervisor of my thesis.

The financial support from Graduate School-782 (GRK-782) and BMBF are greatly acknowledged.

I would like to thank all the peoples in the workshops for their kind cooperation and help.

I am highly indebted to Prof. S. Sampath, Department of Inorganic and Physical Chemistry, Indian Institute of Science, Bangalore for introducing me to the world of surface science. I would also like to thank Prof. B. S. Sherigara, ViceChancellor of Kuvempu University, India for his continual cooperation. My thanks are also due to Dr. Lawrence D'Souza for his kind and encouraging words. My sincere thanks are to my beloved teachers.

My special thanks to my brothers (Manjunatha and Ravi) and friends for their cooperation, support and encouragement during the time of my studies.

My special thanks are also to my fiancée Rashmishree. 


\section{Curriculum Vitae}

Name: $\quad$ Chandrashekara Rajashekara Haramagatti

Date of birth: 17.01.1977

Place of birth: Malligara (India)

Education

1983-1986: Government primary school, Malligara

1986-1990: Government primary school, Anavatti

1990-1993: Government high school, Anavatti

1993-1995: Pre University College (PUC)- Sahyadri Science College,

Shimoga

1995-1998: Bachelor of Sceince (B. Sc), Sahyadri Science College, Shimoga Kuvempu University

1998-2000: Master of Science (M. Sc), Shankaraghatta, Kuvempu University

2000-2003: Research assistant, Indian Institute of Science, Bangalore, India

2003-to date: Research Scholar Institute of Physical Chemistry, University of Göttingen, Germany

\section{Publications}

1. N. Prathima, M. Harini, Neeraj Rai, R. H. Chandrashekara, K.G. Ayappa, S. Sampath, S.K. Biswas* Langmuir 2005, 21, 2364-2374

2. C. R. Haramagatti, A. Islamov, H. Gibhardt, N. Gorski, A. Kuklin, and G. Eckold* Phys. Chem. Chem. Phys. 2006, 8, 994-1000

3. A. Islamov, C. R. Haramagatti, H. Gibhardt, A. Kuklin, and G. Eckold* Physica B 2006, 385-386, 791-794 